\title{
Molecular and Morphological Correlates of Synaptic Vesicle Priming
}

\author{
Dissertation \\ in partial fulfilment of the requirements \\ for the degree "Dr. rer. nat." \\ in the Neuroscience Program \\ at the Georg August University Göttingen, \\ Faculty of Biology
}

submitted by

Cordelia Imig

born in

Wildeshausen, Germany

Göttingen, 2013 



\section{Declaration}

I hereby declare that this thesis was written independently, with no other sources and aids than those cited.

Cordelia Imig,

Göttingen, 17.09.2013 



\section{Table of Contents}

I. Table of Contents

II. List of figures _ VI

III. List of Abbreviation __ VII

IV Abstract__ IX

1. Introduction 1

1.1. Synaptic transmission 1

1.2. Synaptic vesicle docking and priming ___ 3

1.3. Molecular mechanisms underlying docking, priming and fusion __ 6

1.3.1. SNAREs and Munc18-1 _ 6

1.3.2. Munc13s _ 9

1.3.3. $\mathrm{Ca}^{2+}$-dependent activator protein for secretion (CAPS) 12

1.3.4. Synaptotagmin-1__ 14

1.3.5. Complexins _ 15

1.4. Ultrastructural analysis of synaptic vesicle docking___ 17

1.4.1. Classical aldehyde-based fixation methods for electron microscopy __ 17

1.4.2. Cryo-fixation methods for electron microscopy____ 18

1.4.3. Transmission electron microscopy and electron tomography ___ 19

1.5. Aim of the study _ 20

2. Material and Methods__ 23

2.1. Animals_ 23

2.1.1. Mouse lines__ 23

2.2. Molecular Biology__ 23

2.2.1. Materials 23

2.2.1.1. Chemicals and Reagents _ 23

2.2.1.2. Equipment 25

2.2.1.3. Media and buffer 25

2.2.1.4. Vector plasmids_ 25

2.2.1.5. Bacterial Strains 26

2.2.1.6. Kits 26

2.2.1.7. Oligonucleotides _ 27

2.2.2. Methods 28

2.2.2.1. Generation of electrocompetent E. coli cells___ 28

2.2.2.2. Inducing Recombineering Enzymes _ 28

2.2.2.3. Excision of the Neo cassette by arabinose induced Cre expression _ 28

2.2.2.4. Transformation of bacterial strains _ 29

2.2.2.5. Glycerol stocks _ 29

2.2.2.6. BAC DNA preparation 29

2.2.2.7. Plasmid DNA preparation 30

2.2.2.8. Agarose gel electrophoresis and Pulsed field gel electrophoresis __ 30

2.2.2.9. Agarose gel extraction of DNA fragments___ 31

2.2.2.10. Phenol/Chloroform Extraction for large ( $\geq 10 \mathrm{~kb})$ DNA fragments ___ 31

2.2.2.11. Polymerase Chain Reaction __ 31 
2.2.2.12. Dephosphorylation of 5'DNA ends:

2.2.2.13. DNA Ligation and subcloning in TOPO pCR vectors ___ 32

2.2.2.14. Dirty Mini Prep and test digest. (Holmes and Quigley, 1981) __ 33

2.2.2.15. PCR Screens _ 33

2.2.2.16. Cloning strategies for constructs generated and used in this study___ 34

2.2.2.16.1 BAC DNA retrieval vector PL253 34

2.2.2.16.2. IoxP-PGK-EM7-NeobpA-loxP vector PL452_ 34

2.2.2.16.3. FRT-PGK-EM7-NeobpA-FRT-loxP vector PL451__ 34

2.2.2.16.4. Generation of a 3' hybridization probe for Southern Blot Analysis__ 35

2.2.2.16.5. Generation of a 5' hybridization probe for Southern Blot Analysis___ 35

2.2.2.16.6. Generation of a Neo hybridization probe for Southern Blot Analysis __ 35

2.3. Cell Cultures 36

2.3.1. Embryonic Stem Cell Work __ 36

2.3.1.1. Materials__ 36

2.3.1.1.1. Chemicals and Reagents __ 36

2.3.1.1.2. Equipment__ 37

2.3.1.1.3. Media and buffer___ 37

2.3.1.2. Methods _ 37

2.3.1.2.1. Mouse embryonic fibroblast culture __ 37

2.3.1.2.2. Embryonic stem cell culture ___ 38

2.3.1.2.3. ES cell electroporation __ 38

2.3.1.2.4. Picking ES cell colonies __ 38

2.3.1.2.5. Freezing 96-well plates__ 39

2.3.1.2.6. Isolating ES cell DNA on 96-well Plates ___ 39

2.3.1.2.7. Southern Blot (Southern, 1975) ___ 39

2.3.2. Mouse hippocampal Neuron Culture ___ 41

2.3.2.1. Materials__ 41

2.3.2.1.1 Chemicals and Reagents __ 41

2.3.2.1.2. Media and Solutions ___ 41

2.3.2.2. Methods __ 41

2.3.2.2.1. Treatment of coverslips for mouse hippocampal neuron cultures ___ 41

2.3.2.2.2. Primary mouse hippocampal neuron culture __ 42

2.3.3. HEK 293FT cell culture __ 43

2.3.3.1. Materials__ 43

2.3.3.1.1. Chemicals and Reagents _ 43

2.3.3.1.2. Media and buffer___ 43

2.3.3.2. Methods _ 43

2.3.3.2.1. HEK293FT culture 43

2.3.3.2.2. Thawing of HEK293FT cells__ 44

2.3.3.2.3. Passaging of HEK293FT cells _ 44

2.3.3.2.4. Production of lenti-viral particles in HEK293FT cells __ 44

2.3.4. Organotypic Slice Culture __ 45

2.3.4.1. Materials__ 45

2.3.4.1.1. Chemicals and Reagents _ 45

2.3.4.1.2. Equipment_ 45

2.3.4.1.3. Media and buffer___ 45

2.3.1.3. Methods _ 46

2.3.1.3.1. Preparation of organotypic hippocampal slice cultures __ 46

2.4. Biochemistry__ 47

2.4.1. Materials__ 47

2.4.1.1. Chemicals and reagents__ 47 
2.4.1.2. Equipment__ 48

2.4.1.3. Media and buffer _ 48

2.4.1.4. Antibodies _ 48

2.4.2. Methods 49

2.4.2.1. Mouse Brain Homogenate _ 49

2.4.2.2. Cell Culture Homogenization 49

2.4.2.3. Preparation of Proteins Samples for SDS Electrophoresis___ 49

2.4.2.4. SDS-PAGE (Laemmli 1970) and Western Blotting (Towbin 1979; Burnette 1981) 49

2.5. Immunostaining procedures__

2.5.1. Materials 50

2.5.1.1. Chemicals and reagents _ 50

2.5.1.2. Equipment__ 51

2.5.1.3. Media and Solutions __ 51

2.5.1.4. Antibodies 51

2.5.2. Methods 52

2.5.2.1. Immunocytochemistry on autaptic hippocampal neurons__ 52

2.5.2.2. Immunohistochemistry on hippocampal organotypic slices __ 52

2.5.2.3. Imaging__ 52

2.5.2.4. Image processing and analysis___ 53

2.6. Electron Microscopy Techniques __ 54

2.6.1. Materials 54

2.6.1.1. Chemicals and Reagents __ 54

2.6.1.2. Equipment__ 54

2.6.1.3. Media and buffer _ 55

2.6.2. Methods 55

2.6.2.1. High-Pressure-Freezing _ 55

2.6.2.2. Freeze Substitution and EPON embedding _ 55

2.6.2.3. Sectioning and contrasting __ 56

2.6.2.4. Imaging _ 56

2.6.2.5. IMOD Software package ___ 57

2.6.2.6. Two-dimensional (2D) ultrastructural analysis of synaptic morphology __ 58

2.6.2.7. Three-dimensional (3D) electron tomographic (ET) analysis of synaptic vesicle docking _ 59

2.7. Statistical Analysis 61

3. Results_ 62

3.1. Analysis of the morphological correlate of synaptic vesicle priming ___ 62

3.1.1. Ultrastructural organization of mouse hippocampal organotypic slice cultures __ 62

3.1.2. Synaptic vesicle interactions with the active zone membrane ___ 64

3.1.3. Docking Analysis of presynaptic mutants ___ 66

3.1.3.1. The role of the synaptic vesicle priming factors Munc13-1 and Munc13-2 in synaptic vesicle docking _ 66

3.1.3.1.1. 2D-EM analysis of synaptic morphology in Munc13-1/2 DKO neurons__ 66

3.1.3.1.2. 3D-ET analysis of synaptic vesicle docking in Munc13-1/2 DKO neurons _ 67

3.1.3.2. The role of the synaptic vesicle priming factors CAPS-1 and CAPS-2 in synaptic vesicle docking

3.1.3.2.1. 2D-EM analysis of synaptic morphology in CAPS-1/2 DKO neurons ___ 71

3.1.3.2.2. 3D-ET analysis of synaptic vesicle docking in CAPS-1/2 DKO neurons _ _ 72 
3.1.3.3. The role of the t-SNARE SNAP25 in synaptic vesicle docking __ 75

3.1.3.3.1. 2D-EM analysis of synaptic morphology in SNAP25 KO neurons _ 76

3.1.3.3.2. 3D-ET analysis of synaptic vesicle docking in SNAP25 KO neurons _ 76

3.1.3.3.3. SNAP23 does not compensate for the loss of SNAP25

3.1.3.4. The role of the t-SNARE Syntaxin-1 in synaptic vesicle docking ___ 81

3.1.3.4.1. 2D-EM analysis of synaptic morphology in Syntaxin-1A KO/ $-B^{\text {YFP }}$

neurons

3.1.3.4.2. 3D-ET analysis of synaptic vesicle docking in Syntaxin-1A KO/ -B

neurons _ 83

3.1.3.5. The role of the v-SNARE Synaptobevin-2 in synaptic vesicle docking ___ 85

3.1.3.5.1. 2D-EM analysis of synaptic morphology in Synaptobrevin-2 KO neurons _ 86

3.1.3.5.2. 3D-ET analysis of synaptic vesicle docking in Synaptobrevin-2 KO neurons 86

3.1.3.5.3. Synaptobrevin-1 can compensate for the loss of Synaptobrevin-2 in synapses

3.1.3.6. The role of the vesicular $\mathrm{Ca}^{2+}$-sensor Synaptotagmin-1 in synaptic vesicle docking 92

3.1.3.6.1. 2D-EM analysis of synaptic morphology in Synaptotagmin-1 KO neurons _ 93

3.1.3.6.2. 3D-ET analysis of synaptic vesicle docking in Synaptotagmin-1 KO neurons 93

3.1.3.7. The role of Complexins in synaptic vesicle docking__ 96

3.1.3.7.1. 2D-EM analysis of synaptic morphology in Complexin-1/2/3 TKO neurons_ 97

3.1.3.7.2. 3D-ET analysis of synaptic vesicle docking in Complexin-1/2/3 TKO neurons 98

3.1.3.8. The impact of the synaptic vesicle size on membrane-attachment ___ 101

3.1.3.9. SNARE mutants accumulate LDCVs in presynaptic terminals __ 102

3.2. Generation and characterization of a Munc13-1 conditional knock-out (CKO) mouse line 105

3.2.1. Generation of a CKO targeting vector__ 105

3.2.1.1. Retrieving of BAC DNA fragment into the PL253 vector _ 107

3.2.1.2. Targeting of the first loxP site upstream of Munc13-1 exon $21 \_108$

3.2.1.3. Targeting of the second loxP downstream of Munc13-1 exon 21

3.2.2. Generation of recombinant ES cells and mice ___ 110

3.2.3. Genotyping Strategy ___ 113

3.2.4. Characterization of the Munc13-1 CKO mouse line ___ 114

4. Discussion__ 117

4.1. Methodological aspects in studying synaptic vesicle docking ___ 117

4.1.1. The organotypic slice culture system for studying lethal mouse mutants ___ 117

4.1.2. Studying synaptic vesicle docking using cryo-fixation methods for EM___ 118

4.2. A common pool of docked and primed synaptic vesicles?

4.3. The role of the priming proteins Munc13/CAPS in synaptic vesicle docking __ 122

4.3.1. Munc13s__ 122

4.3.2. CAPS 125

4.4. A role for all three neuronal SNAREs in synaptic vesicle docking and priming __ 129

4.5. Loss of individual SNARE proteins can be partially compensated ___ 130

4.6. Increased vesicle sizes do not cause the deficiency in synaptic vesicle membraneattachment

4.7. Can trans-SNARE complexes be stabilized in vivo? 134

4.8. Can partially assembled trans-SNARE complexes be stabilized by Synaptotagmin-1 or Complexins?

4.9. A regulatory, but not essential role of Synaptotagmin-1 in synaptic vesicle docking/ priming

4.10. Enlarged synaptic vesicles in Synaptobrevin-1, SNAP25 and Munc13 KO synapses 
5. Summary 148

6. Supplementary Figures __ 150

7. Bibliography 152

Acknowledgements 170 List of publications 172 


\section{List of figures}

Figure 1.1. The synaptic vesicle cycle at excitatory synapses 3

Figure 1.2. Schematic representations of domain structures of key presynaptic proteins 6

Figure 2.1. Two-dimensional ultrastructural analysis of synaptic morphology 59

Figure 3.1. Ultrastructural organization of mouse hippocampal organotypic slice cultures. 63

Figure 3.2. Direct interactions of synaptic vesicles with the active zone membrane 65

Figure 3.3. Two-dimensional ultrastructural analysis of synaptic morphology in Munc13-1/2 DKO

neurons 68

Figure 3.4. Three-dimensional electron tomographic analysis of synaptic vesicle docking in Munc13-1/2 DKO neurons $\quad 70$

Figure 3.5. Two-dimensional ultrastructural analysis of synaptic morphology in CAPS-1/2 DKO neurons 73

Figure 3.6. Three-dimensional electron tomographic analysis of synaptic vesicle docking in CAPS-1/2 DKO neurons

Figure 3.7. Two-dimensional ultrastructural analysis of synaptic morphology in SNAP25 KO neurons

Figure 3.8. Three-dimensional electron tomographic analysis of synaptic vesicle docking in SNAP25 KO neurons

Figure 3.9. Two-dimensional ultrastructural analysis of synaptic morphology in Syntaxin-1A KO/ -B ${ }^{\text {YFP }}$ neurons

Figure 3.10. Three-dimensional electron tomographic analysis of synaptic vesicle docking in Syntaxin-1A KO/ $-B^{\mathrm{YFP}}$ neurons

Figure 3.11. Two-dimensional ultrastructural analysis of synaptic morphology in Synaptobrevin-2 KO neurons

Figure 3.12. Three-dimensional electron tomographic analysis of synaptic vesicle docking in Synaptobrevin-2 KO neurons

Figure 3.13. Quantitative analysis of synaptic vesicle docking in Synaptobrevin-2 KO neurons 90

Figure 3.14. Two-dimensional ultrastructural analysis of synaptic morphology in Synaptotagmin-1 $\mathrm{KO}$ neurons

Figure 3.15. Three-dimensional electron tomographic analysis of synaptic vesicle docking in Synaptotagmin-1 KO neurons

Figure 3.16. Two-dimensional ultrastructural analysis of synaptic morphology in

Complexin-1/2/3 TKO neurons

Figure 3.17. Three-dimensional electron tomographic analysis of synaptic vesicle docking in Complexin-1/2/3 TKO neurons

Figure 3.18. Enlarged vesicle diameters do not impair synaptic vesicle docking 102

Figure 3.19. SNARE mutants accumulate LDCVs in presynaptic terminals 103

Figure 3.20. Generation of a Munc13-1 conditional knock-out targeting vector 107

Figure 3.21. Generation and characterization of Munc13-1 conditional knock-out mice 112

Figure 3.22. Electrophysiological and morphological characterization of pFUGW-iCre infected Munc13-1 Flp hippocampal autaptic neurons

$\begin{array}{lll}\text { Figure 4.1. } & \text { The synaptic vesicle cycle at excitatory synapses } & 128\end{array}$

Figure 4.2. Molecular mechanisms of synaptic vesicle docking/priming 143

Figure S1. Light microcopic analysis of SNAP23 and VGLUT1 immunoreactivity in SNAP25 KO organotypic hippocampal slices 150

Figure S2. Light microcopic analysis of Syb-1 and VGLUT1 immunoreactivity in Syb-2 KO organotypic hippocampal slices 


\section{List of Abbreviation}

2D

3D

AMPA

$A m p^{r}$

BAC

Baiap-3

BoNT C/N

bpa

BSA

$\mathrm{Ca}^{2+}$

CAPS

C. elegans

$\mathrm{CKO}$

$\mathrm{CO}_{2}$

Cre

C-terminal

Drosophila

DAG

${ }_{\text {dd }} \mathrm{H}_{2} \mathrm{O}$

DIV

DKO

DNA

E18

EM

EPSC

ES cells

ET

floxed

FLP

FRET

FRT

GABA

HEK293FT

HRP

HSV-TK

IPSC

$\mathrm{Kan}^{\mathrm{r}}$

$\mathrm{KO}$

LB

LDCV

MAP-2

MEF

MEPSC

MHD

mIPSC

mOsm

$\mathrm{NeO}$

$\mathrm{nm}$

NMDA

$\mathrm{N}$-terminal

$\mathrm{OD}_{600}$
Two-dimensional

Three-dimensional

a-Amino-3-hydroxy-5-methyl-4-isoxazolepropionic acid

Ampicilin resistent

Bacterial artificial chromosome

Brain-specific angiogenesis inhibitor-associated protein 3

Botulinum neurotoxin serotype $\mathrm{C} / \mathrm{N}$

Bovine growth hormone polyadenylation site

Bovine serum albumin

Calcium

$\mathrm{Ca}^{2+}$-dependent activator protein for secretion

Caenorhabditis elegans

Conditional knockout

Carbon dioxide

Cyclization recombination

Carboxy-terminal

Drosophila melanogaster

Diacylglycerol

Double-distilled water

Days in vitro

Double knockout

Deoxyribonucleic acid

Embryonic day 18

Electron microscopy

Excitatory postsynaptic current

Embryonic stem cells

Electron tomography

Flanked by loxP sites

Flippase

Förster resonance energy transfer

Flippase recombinase target sequences

Y-Aminobutyric acid

Human embryonic kidney 293FT cell line

Horseradish peroxide

Herpes simplex virus thymidine kinase

Inhibitory postsynaptic current

Anamycin resistent

Knockout

Luria broth

Large-dense core vesicle

Microtubule-associated protein 2

Mouse embryonic fibroblasts

Miniture excitatory postsynaptic currents

Munc13 homology domain

Miniture inhibitory postsynaptic current

Milliosmole

Neomycin resistence gene

Nanometer

$N$-Methyl-D-aspartic acid

Amino-terminal

Optical density measured at $600 \mathrm{~nm}$ wavelength 


$\begin{array}{ll}\mathrm{OsO}_{4} & \text { Osmium tetroxide } \\ \text { PO } & \text { Postnatal day } 0 \\ \text { PBS } & \text { Phosphate buffer saline } \\ \text { PCR } & \text { Polymerase chain reaction } \\ \text { PDBu } & \text { Phorbol 12,13 dibutyrate } \\ \text { PFA } & \text { Paraformaldehyde } \\ \text { PGK } & \text { Phosphoglycerate kinase } \\ \text { PIP } & \text { Phosphatidylinositol phosphate } \\ \text { PIP } & \text { Phosphatidylinositols 4,5 bisphosphate } \\ \text { PSD } & \text { Postsynaptic density } \\ \text { PVr } & \text { Vesicular release probability } \\ \text { recombineering } & \text { Recombination-mediated genetic engineering } \\ \text { RIM } & \text { Rab3a-interacting molecule } \\ \text { rpm } & \text { Revolutions per minute } \\ \text { RRP } & \text { Readiliy-releasable pool } \\ \text { SEM } & \text { Standard error of the mean } \\ \text { SV } & \text { Synaptic vesicle } \\ \text { TKO } & \text { Triple knockout } \\ \text { TMR } & \text { Transmembrane region } \\ \text { t-SNARE } & \text { Target-membrane SNARE } \\ \text { UNC } & \text { Uncoordinated } \\ \text { VGLUT1 } & \text { Vesicular glutamate transporter 1 } \\ \text { V-SNARE } & \text { Vesicular SNARE } \\ \text { WT } & \text { Wild-type } \\ \text { YFP } & \text { Yellow fluorescent protein } \\ & \end{array}$


Excitation-secretion coupling at nerve cell synapses is a sub-millisecond process that entails the transduction of an electrical stimulus into synaptic vesicle fusion. Before fusion, synaptic vesicles are physically docked to the presynaptic active zone membrane and functionally primed to become fusion competent. In response to an increase in intracellular calcium concentration after the arrival of an action potential, primed vesicles fuse with the plasma membrane and release their neurotransmitter content into the synaptic cleft. Recent studies combining cryo-fixation methods and three-dimensional electron microscopy analysis proposed that synaptic vesicle docking and priming steps may not reflect independent mechanisms, but rather describe the same molecular process namely full or partial soluble $\mathrm{N}$-ethylmalemide-sensitive factor attachment protein receptor (SNARE) complex formation initiated by members from the UNC-13/Munc13 protein family. However, other studies have challenged the notion of SNARE complex assembly prior to the calcium triggering step in the release process.

In the present study, a combination of organotypic hippocampal slice culture, highpressure freezing, freeze substitution and electron tomography was used to reinvestigate the role of key synaptic proteins in synaptic vesicle docking in glutamatergic hippocampal spine synapses. This method enables the analysis of synaptic parameters in an in-situ-like setting using lethal mouse mutants that do not survive birth. Loss or reduction of components of the molecular priming machinery, namely Munc13 or CAPS proteins, and of the individual neuronal SNARE proteins Synaptobrevin-2, Syntaxin-1 and synaptosome-associated protein of $25 \mathrm{kDa}$ (SNAP25) caused severe defects in synaptic vesicle membrane-attachment in this experimental setting. Moreover, loss of the calcium $\left(\mathrm{Ca}^{2+}\right)$-sensor Synaptotagmin-1, causes a decrease in vesicle numbers in presynaptic terminals in comparison to control synapses and a reduction in membrane-proximal (loosely tethered) and docked vesicles. However, the reduction in the number of membrane-attached synaptic vesicles was milder than it was observed in the absence of the vesicular SNARE ptrotein Synaptobrevin-2, indicating that Synaptotagmin-1 might have a regulatory (e.g. tethering or clamping) but not essential role in synaptic vesicle docking in neurons. Complexin-deficient synapses exhibited no changes in the number of membrane-attached synaptic vesicles, a finding that supports a facilitatory rather than inhibitory role of Complexins prior to synaptic vesicle fusion. These findings indicate that synaptic vesicle membrane-attachment, synaptic vesicle priming and (partial) SNARE complex assembly are respective morphological, functional and molecular manifestations of the same process. 



\section{Introduction}

\section{1. \\ Synaptic transmission}

The brain is a highly complex network composed of billions of neurons that communicate with each other in a regulated manner in order to control a variety of processes including motor control, sensory information processing, as well as learning and memory functions. Along a neuron, information is conveyed as electrical signals, however neurons form highly specialized contacts at chemical synapses that mediate information transduction and processing between a presynaptic and a postsynaptic compartment.

An electrical signal (action potential) that arrives at the presynaptic terminal is converted into a chemical response by the release of messenger molecules (neurotransmitters) at the active zone, a process often referred to as excitation-secretion coupling (reviewed in Wojcik \& Brose 2007). This process is very fast and works with high fidelity and endurance, which requires local recycling of the neurotransmitter and the molecular release machinery in the presynaptic terminal (reviewed in Jahn and Fasshauer, 2012; Südhof, 1995, 2004). Neurotransmitter molecules are stored in small, spherical, lipidbound organelles called synaptic vesicles. Synaptic vesicles bud off from recycling organelles, termed endosomes, and neurotransmitter molecules are filled into the vesicles by neurotransmitter transporters using the energy of a proton gradient across the vesicle membrane. Neurotransmitter-containing synaptic vesicles are transported to the active zone, a highly specialized region at the presynaptic plasma membrane, where neurotransmitter release takes place. At the active zone, synaptic vesicles attach to the plasma membrane in a process called "docking" and become fusion-competent in a functional "priming" process (reviewed in Wojcik \& Brose 2007). The common view is that only a subset of the docked synaptic vesicles becomes primed, thus forming a readilyreleasable pool (RRP) of vesicles which can be released in response to the arrival of an action potential.

The arrival of the electrical signal at the presynaptic terminal causes a change in the electrical potential across the plasma membrane which initiates the rapid opening of voltage-gated $\mathrm{Ca}^{2+}$-channels clustered at the active zone membrane. As a consequence, $\mathrm{Ca}^{2+}$ ions flow into the presynaptic terminal and cause a local increase in the $\mathrm{Ca}^{2+}$ concentration. This increase in intracellular $\mathrm{Ca}^{2+}$ levels can be sensed by the molecular release machinery and functions as the signal to trigger fusion of synaptic vesicles with the plasma membrane in a process called exocytosis. The exocytosis of vesicles is thought to comprise multiple intermediate states (reviewed in Kasai et al., 2012): (i) 
membrane-attached vesicles hemifuse with the plasma membrane as the outer leaflet of the vesicular phospholipid bilayer merges with the inner leaflet of the membrane bilayer, (ii) a small fusion pore connecting the vesicular lumen with the extracellular space is formed as distal leaflets of the lipid bilayers intermingle, and (iii) the fusion pore expands and the vesicle collapses into the plasma membrane. As a result, the neurotransmitter content of fusing synaptic vesicles is released from the presynaptic terminal.

The released neurotransmitter molecules diffuse through the extracellular space between the pre- and postsynaptic compartment, referred to as the synaptic cleft, until they reach the postsynaptic membrane. Upon binding to specific receptors, neurotransmitters activate, inhibit or modulate the activity of the postsynaptic cell. To guarantee the constant availability of synaptic vesicles, especially during high synaptic activity, not only neurotransmitter and molecular components of the presynapse, but also the synaptic vesicle membranes need to be recycled. The retrieval of vesicles from the plasma membrane is achieved by endocytosis (Heuser and Reese, 1973; reviewed in Südhof, 1995, 2004). Different pathways of endocytosis have been proposed, with the most widely accepted one being clathrin-mediated endocytosis (Dittman and Ryan, 2009; Heuser and Reese, 1973; Hua et al., 2011a). In this process, synaptic vesicle proteins incorporated into the plasma membrane during vesicle fusion are recognized by specific adaptor proteins, which in turn recruit a local scaffold of clathrin molecules. Clathrin molecules induce inward curvature of the membrane (pits) which, with the concerted action of dynamin proteins, can be pinched off to form a new vesicular organelle. Alternative routes for endocytosis described include a direct coupling of exo- and endocytosis called kissand-run, whereby synaptic vesicles return to the cytoplasm following neurotransmitter release without fully collapsing into the plasma membrane, and a process called bulkmembrane retrieval, where large portions of membrane are internalized during high synaptic activity. However, these processes are less well studied and often controversially discussed (Aravanis et al., 2003; Cheung et al., 2010; Gandhi and Stevens, 2003; Granseth et al., 2006, 2009; Watanabe et al., 2013; Wenzel et al., 2012; Zhang et al., 2009).

Action-potential evoked synaptic vesicle fusion at synapses is a very fast, endurable and tightly-regulated process that is controlled by a wide range of proteins to secure highspeed and reliability even during sustained activity. The active zone is characterized by a unique composition of cytoskeletal elements, voltage gated $\mathrm{Ca}^{2+}$-channel clusters and proteins that regulate synaptic vesicle fusion and therefore secure temporal and spatial accuracy in the release process (reviewed in Südhof 2012). 


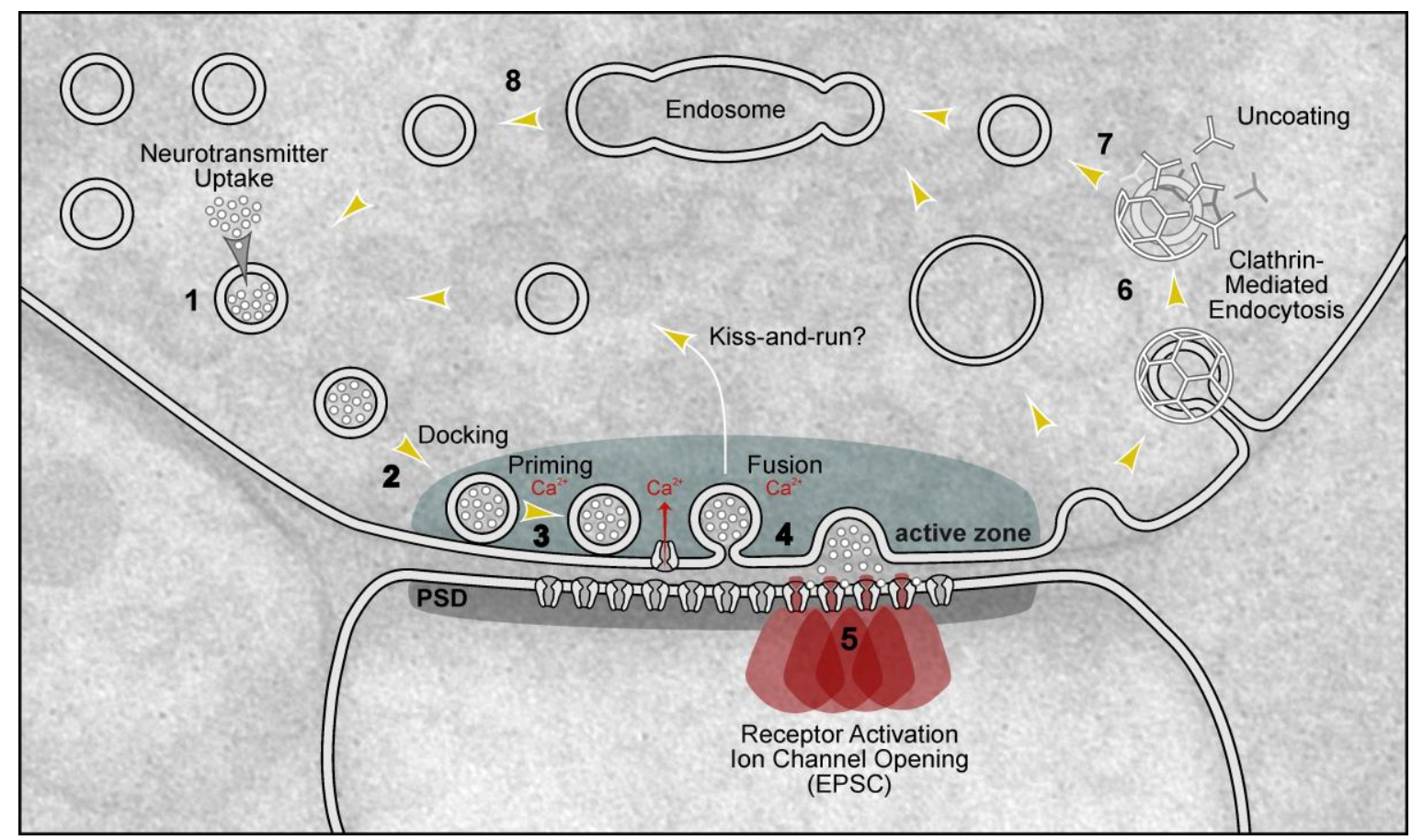

Figure 1.1. The synaptic vesicle cycle at excitatory synapses

Synaptic vesicles are locally recycled in presynaptic terminals. Synaptic vesicles are filled with the neurotransmitter (here e.g. glutamate) (1) and translocated to the active zone. There, synaptic vesicles become physically attached to the plasma membrane (2) and undergo a $\mathrm{Ca}^{2+}$-regulated priming process to acquire fusion-competence (3). In response to the arrival of an action potential and an increase in $\mathrm{Ca}^{2+}$-concentration, primed synaptic vesicles can fuse with the plasma membrane (4) and release their neurotransmitter content into the synaptic cleft. Neurotransmitter molecules diffuse through the synaptic cleft to mediate receptor ion channel opening and allow ion influx generating an excitatory postsynaptic current (EPSC) (5). Synaptic vesicle membranes are recycled in a process called endocytosis (6). The best-described pathway is clathrin-mediated recycling, in which clathrin-coated vesicles bud off from the membrane, become uncoated and fuse with early endosomes (7). Kiss-and-run exocytosis and bulk membrane retrieval are possible alternative, clathrin-independent recycling routes. Schematic provided by $\mathrm{Dr}$. B. Cooper; based on (Jahn and Fasshauer, 2012)

\subsection{Synaptic vesicle docking and priming}

Studying neurotransmitter release from neurons requires well-defined settings, which enable the assessment of physiological and morphological parameters and an easy way to monitor, stimulate or pharmacological manipulate the release process. The vast majority of studies characterizing the physiological function of proteins in synaptic transmission have therefore been performed in neuronal culture systems, in which the experimental conditions can be precisely controlled and the electrophysiological properties of neurons can be measured by using the patch-clamp technique (Neher and Sakmann 1976). However, morphological or ultrastructural features of synaptic terminals require the use of a high-resolution microscopy approach, called electron microscopy 
(EM) to be able to resolve presynaptic vesicles and cytoplasmic structures in the nanometer range (Verhage and Sørensen 2008).

A special culture method is a low density culture of neurons plated on astrocytic feeder islands that will result in neurons that form synapses onto themselves (autapses) in the absence of neighboring cells (Bekkers, 1991; Burgalossi et al., 2012; Pyott and Rosenmund, 2002). In this experimental system, application of hypertonic sucrose solution has been shown in electrophysiological experiments to deplete the pool of fusioncompetent, primed synaptic vesicles (RRP) in an as yet unknown manner (Rosenmund and Stevens, 1996; Stevens and Tsujimoto, 1995). In these early studies, calculations of the measured postsynaptic electrical responses after application of hyperosmotic solution indicated that a depletion of the entirety of synaptic vesicles in a neuron would result in a much greater response than measured and that therefore possibly only a subset of synaptic vesicles would be released in such an experiment. It was suggested that this pool of synaptic vesicles would very likely be already close, or in contact with, the active zone membrane to ensure fast fusion after the arrival of the action potential. Using aldehyde-based chemical fixation methods for electron microscopic analysis of synaptic ultrastructure, C. Stevens and colleagues were able to confirm a tight correlation between the number of membrane-attached or docked synaptic vesicles and the active zone area. Moreover, the number of docked vesicles was found to correlate approximately with the calculated quantal size of the measured RRP after hypertonic sucrose solution application in autaptic neurons (Murthy and Stevens, 1999; Rosenmund and Stevens, 1996; Schikorski and Stevens, 1997).

A "docked" pool of synaptic vesicles therefore describes synaptic vesicles that are in direct contact with the active zone membrane at synaptic release sites, as seen in electron micrographs. However, synaptic vesicle "priming" is a term that arose from the hypothesis that a multistep-molecular mechanism preceding $\mathrm{Ca}^{2+}$-triggered release would be much too slow to allow synaptic vesicle release at high frequency for long durations (reviewed in Südhof 1995). It was shown that during high-frequency stimulation for 15 minutes, the number of synaptic vesicles observed in contact with the presynaptic release sites in electron micrographs from lamprey reticulospinal axon synapses did not decrease, whereas electrophysiologically, synaptic release slows down much earlier (reviewed in Südhof, 1995; Wickelgren et al., 1985). Moreover, animals that were lacking presynaptic proteins exhibited massive physiological defects in basic neurotransmitter release characteristics accompanied by an almost completely depleted RRP of primed vesicles, but displayed normal numbers of membrane-attached synaptic vesicles in electron 
micrographs (Aravamudan et al., 1999; Augustin et al., 1999a; Richmond et al., 1999; Varoqueaux et al., 2002). These findings indicated that instead of the synaptic vesicle docking step, a subsequent $\mathrm{Ca}^{2+}$-dependent molecular priming step that renders the synaptic vesicles fusion-competent might be rate-limiting (reviewed in Südhof 1995).

Conventional sample preparation techniques for EM employing aldehyde-based chemical fixation are subject to certain experimental constraints: (i) the speed of sample fixation is limited by the diffusion of chemical fixatives through the tissue (requiring minutes to hours), and (ii) artefacts introduced by dehydration steps at room-temperature alter the spatial relationships and ultracellular integrity of cellular organelles (e.g. shrinkage). Recent improvements in sample preparation techniques employing rapid cryo-fixation methods resulted in superior ultrastructural preservation coupled with a dramatically improved temporal resolution (milliseconds), therefore enabling a more reliable analysis of membrane-attached vesicles in presynaptic protein null mutants (See 1.4. Ultrastructural analysis of synaptic vesicle docking). Studies using these methods indicated that synaptic vesicle docking and priming might not be independent processes.

A SNAP25 (206 aa)

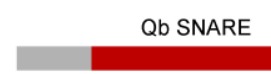
QC SNARE

B Syntaxin-1A
$(288 \mathrm{aa})$ N-pep Qa SNARE (H3 Domain) TMR

C Synaptobrevin-2 (116 aa)

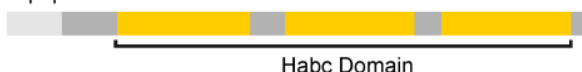

Habc Domain

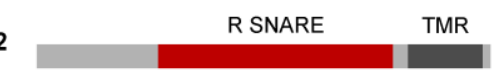

D Munc13-1

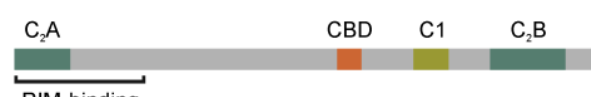
MHD1

E

CAPS-1 (1355 aa)

$\mathrm{C}_{2} \quad \mathrm{PH}$

MUN Domain

RIM-binding

MHD1

F Synaptotagmin-1 (421 aa)

G $\underset{(134 \mathrm{aa})}{\text { Complexin-1 }}$ TMR $\mathrm{C}_{2} \mathrm{~A}$ $\mathrm{C}_{2} \mathrm{~B}$

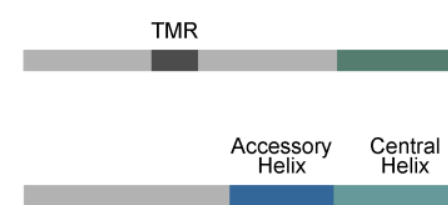


Figure 1.2. Schematic representations of domain structures of key presynaptic proteins

The domain structures of the SNARE (soluble N-ethylmalemide-sensitive factor attachment protein receptor) proteins SNAP25 (A), Syntaxin-1A (B) and Synaptobrevin-2 (C), the priming proteins Munc13-1 (D) and CAPS-1 (E), the $\mathrm{Ca}^{2+}$-sensor Synaptotagmin-1 (F), and Complexins (G). The number of amino acids (aa) are indicated for each protein. $C_{1}$ domain in Munc13-1 (light green), $C_{2}$ domains in Munc13-1, CAPS-1 and Synaptotagmin-1 (dark green), CBD in Munc13-1 (Calmodulinbinding domain, orange), Habc domain of Syntaxin-1 (three a-helices, yellow), MHD in Munc13-1 and CAPS-1 (Munc homology domain, dark red) N-pep of Syntaxin-1 (amino-terminal peptide, light grey), PH domain (Pleckstrin homology, violet), SNARE motifs in SNAP25, Syntaxin-1, Synaptobrevin-2 (red; $\mathrm{Q}$ = central glutamine residue; $\mathrm{R}=$ central arginine residue), TMR (transmembrane region, dark grey). The domain structures are not drawn to scale. Schematic provided by Dr. Ben Cooper; based on (Kasai et al. 2012).

\subsection{Molecular mechanisms underlying docking, priming and fusion}

\subsubsection{SNAREs and Munc18-1}

Hundreds of proteins have been described to participate in the tight regulation of the different steps of synaptic vesicle release. The key players in membrane-fusion reactions, however, are SNARE proteins that form the exocytotic core complex for membrane fusion (reviewed in Jahn and Fasshauer, 2012; Jahn and Scheller, 2006). The family of the (SNARE) proteins is characterized by a stretch of 60-70 amino acids referred to as the SNARE motif, which is highly conserved and undergoes regulated assembly to form stable ternary SNARE-complexes (Fasshauer et al., 1998; Kloepper et al., 2007, 2008). The family of synaptic SNARE molecules include the synaptic vesicle protein Synaptobrevin-2 (vesicular, v-SNARE) and the two plasma-membrane residing molecules Syntaxin-1 and SNAP25 (target-membrane, t-SNAREs). In the course of the fusion reaction, the three molecules form a parallel four- $\alpha$-helical bundle by interaction of their respective SNARE motifs (Poirier et al., 1998; Sutton et al., 1998).

Within the assembled SNARE complex, the side chains of the individual $\alpha$-helices form hydrophobic interactions between each other, with the only exception being a central amino acid layer that consists of four interacting charged amino residues, one arginine $(R)$ contributed by Synaptobrevin-2 (R-SNARE), one glutamine (Qa) from Syntaxin-1 and two glutamines (Qb, Qc) from SNAP25 (Q-SNAREs), which contributes two SNARE motifs (Fasshauer et al., 1998). Despite the highly conserved nature of SNARE motifs, the three neuronal SNAREs vary in their domain structure. Synaptobrevin-2 and Syntaxin-1 are anchored to the vesicle or plasma membrane by a single carboxy (C) -terminal transmembrane region (TMR) that is connected to the SNARE motif by a short linker. SNAP25, however, varies from this structure as its two SNARE motifs are connected by a 
short, hydrophobic linker that contains palmitoylated cysteines, a post-translational protein modification, which allows plasma membrane-anchoring without a TMR (reviewed in Hong, 2005). Whereas, Synaptobrevin-2 and SNAP25 lack any additional domains, Syntaxin-1 contains a short amino (N)-terminal peptide and an additional $\mathrm{N}$-terminal domain, which forms a three $\alpha$-helical bundle (Habc domain) that is linked to, and folds onto, the SNARE motif of Syntaxin-1 and renders the molecule in a "closed"-conformation, most-likely by the interaction with Munc18-1 (Dulubova et al., 1999; Fernandez et al., 1998).

The most widely accepted model for SNARE complex zippering is that it starts at the Nterminus of the SNARE motifs by interaction of the v-SNARE Synaptobrevin-2 with the tSNAREs Syntaxin-1 and SNAP25 in a trans conformation and progresses towards the Cterminal membrane-anchors (reviewed in Kasai et al., 2012; Pobbati et al., 2006; Stein et al., 2009). This process will pull the synaptic vesicle and the plasma membrane closer together. Full zippering of the ternary SNARE complex has been shown to release a high amount of energy, which can possibly be conducted through the linkers to the TMR, inducing destabilization of the membrane and fusion of the bilayers (Li et al., 2007; Stein et al., 2009). After the membrane-fusion reaction, the assembled SNARE complex resides in the plasma membrane in a low energy cis conformation, which is subsequently disassembled by the AAA-ATPase NSF (N-ethylmaleimide sensitive factor) together with its SNAP (soluble NSF attachment proteins) adaptor molecules (Burgalossi et al., 2010; reviewed in Jahn and Scheller, 2006). In vitro studies that used reconstituted SNARE complex formation assays, proposed a model, according to which the two t-SNAREs Syntaxin-1 and SNAP25 form a highly reactive intermediate acceptor complex on the membrane (Fasshauer and Margittai, 2004; Pobbati et al., 2006). This complex might be stabilized in vivo by regulatory proteins prior to Synaptobrevin-2 engagement.

Null mutant mice of the neuronal SNAREs Synaptobrevin-2 and SNAP25 die prior to birth (Schoch et al., 2001; Washbourne et al., 2002). Neurons lacking the v-SNARE Synaptobrevin-2 exhibit an $85 \%$ decrease in spontaneous synaptic vesicle fusion events, which is reflected by a reduced frequency of spontaneous miniature excitatory postsynaptic currents (mEPSC) and a 90\% reduction in the size of the RRP of primed synaptic vesicles measured after hypertonic sucrose application (Schoch et al. 2001). However, action potential evoked, $\mathrm{Ca}^{2+}$-dependent release, measured in the size of the evoked excitatory postsynaptic current (EPSC) recorded after stimulation, is almost completely abolished (Schoch et al. 2001). Moreover, Synaptobrevin-2 KO mice exhibit a significant increase in the vesicle diameter, which was interpreted as a possible 
endocytosis defect, although neurons showed no additional changes in synaptic morphology or in the number of docked synaptic vesicles in electron micrographs (Deák et al., 2004). Low-density cultures of neurons lacking SNAP25 exhibit neurodegeneration accompanied by cell death and synapse loss. However, high-density cultures were shown to prolong neuronal survival over three weeks' time (Bronk et al., 2007; Washbourne et al., 2002). SNAP25 KO neurons are characterized by a strong reduction in the mEPSC frequency accompanied by a slight decrease in the mEPSC amplitude. Evoked, $\mathrm{Ca}^{2+}-$ dependent neurotransmitter release measured by field stimulation revealed that $\sim 70 \%$ of the measured neurons failed to respond after stimulation and the remaining cells only exhibited an EPSC that was dramatically decreased in amplitude. The size of the RRP measured by hypertonic sucrose application was reduced to $12 \%$ of the control value. However no alterations in synaptic morphology or in the number of docked synaptic vesicles could be detected in EM analyses (Bronk et al., 2007).

A mouse mutant deficient for both Syntaxin-1A and B isoforms has only recently become available, but mutant neurons of these mice degenerate and do not survive the first few days of culture (Rosenmund and Südhof, unpublished data). Proteolytic cleavage of Syntaxin-1 after viral expression of Botulinium neurotoxin serotype C (BoNT/C) in cultured neurons revealed a robust reduction in the mEPSC frequency and in the evoked EPSC response, with no change in the number of membrane-attached synaptic vesicles (de Wit et al., 2006). A recent study introduced a knock-in mouse line for Syntaxin-1B, in which the protein is fused to a yellow fluorescent protein (YFP) in the Syntaxin-1A KO background, and which exhibits a significant reduction in Syntaxin-1 levels in neurons (Arancillo et al., 2013; Gerber et al., 2008). The reduction of Syntaxin-1 protein levels causes embryonic lethality and severe physiological phenotypes with a reduction of the RRP size by $65 \%$ and of the EPSC size by $80 \%$ with respect to control levels in hippocampal autaptic neurons. Moreover, these electrophysiological alterations are accompanied by changes in the synaptic vesicle distribution (Arancillo et al. 2013). Of note, the reduction of Syntaxin-1 levels also caused a $25 \%$ reduction in the expression levels of Munc18-1, a neuronal protein of the Sec1/Munc18 (SM) family of trafficking molecules and the homologue of $C$. elgans UNC-18 (Arancillo et al. 2013). Munc18-1 KO mice die at birth and exhibit a complete loss of synaptic neurotransmission, and mutant neurons degenerate within days in culture (Heeroma et al., 2004; Verhage, 2000). Moreover, Munc18-1 KO neurons exhibit a 70\% reduction of Syntaxin-1 levels, indicating that Munc18-1 has a role in stabilizing the t-SNARE Syntaxin-1. 
The fact that mouse mutants lacking individual SNARE proteins exhibit massive physiological impairments in neurotransmission and a great decrease in the size of the RRP of primed vesicles measured by hypertonic sucrose solution, led to the hypothesis that the formation of the SNARE complex might indeed be the molecular process underlying synaptic vesicle priming (Arancillo et al., 2013; Schoch et al., 2001). In vitro, it has been shown that SNARE complex formation alone seems to be sufficient to mediate membrane fusion and that the fusion efficiency can further be enhanced by Munc18-1, indicating that most likely additional regulatory proteins are crucial for the speed of $\mathrm{Ca}^{2+}$ evoked membrane fusion in vivo (reviewed in Rizo and Südhof, 2012; Shen et al., 2007; Weber et al., 1998). The role of the individual SNARE molecules in synaptic vesicle docking in neurons has not yet been assessed using improved cryo-preservation methods for EM. The outcome of such experiments could help to clarify the role of the SNARE complex prior to synaptic vesicle fusion.

\subsubsection{Munc13s}

Munc13s are major components of the molecular priming machinery. They are large ( $200 \mathrm{kDa}$ ) proteins that represent the mammalian homologues of C. elegans UNC-13 proteins, which were first identified in a genetic screen for mutants with defects in synaptic transmission (Brenner, 1974). In C. elegans, two UNC-13 isoforms are expressed from the unc13 gene, termed UNC-13L (long, previously: LR) and UNC-13S (short, previously: $\mathrm{MR}$ ), which have highly conserved $\mathrm{C}$-termini, but differ in their $\mathrm{N}$-terminal domain structure (Hu et al., 2013; Kohn et al., 2000). Mammals express at least five different Munc13 isoforms, Munc13-1, bMunc13-2, ubMunc13-2, Munc13-3, Munc13-4 and brain-specific angiogenesis inhibitor-associated protein 3 (Baiap-3), all characterized by a highly conserved C-terminus (Koch et al., 2000). Munc13-1 is widely expressed throughout the central nervous system and is the most dominant isoform present in most synapses. Munc13-2 mRNA expression can be found especially throughout the cortex, hippocampus and cerebellum, whereas Munc13-3 is primarily expressed in the cerebellum (Augustin et al., 1999b, 2001). Munc13-1, bMunc13-2 and Munc13-3 are brain specific isoforms, whereas ubMunc13-2 is more ubiquitously expressed in a great variety of tissues (Augustin et al., 1999b). Munc13-4 is mainly expressed in peripheral tissues and can be detected in the lung, the spleen and testes as well as in cells of the immune system, where it functions in regulated exocytosis of granules in cytotoxic $T$ lymphocytes. Mutations of the human Munc13-4 gene are linked to the familial immune disease hemophagocytic lymphohistiocytosis subtype 3 (Dudenhöffer-Pfeifer et al., 2013; Feldmann et al., 2003; Koch et al., 2000). The brain-specific molecule Baiap-3 is highly 
expressed in the amygdala, the hypothalamus and the periaqueductal gray matter and has been associated with fear and anxiety pathways (Koch et al., 2000; Wojcik et al., 2013).

Characteristic of all members of the UNC-13/Munc13 protein family is the highly conserved C-terminal region which contains two Munc13 homology domains (MHD) connected by a linker (Koch et al., 2000). This region, referred to as the MUN domain, has been shown to weakly interact with the N-terminus of Syntaxin-1 (Betz et al., 1997; Ma et al., 2011, 2013). It has been shown that the MUN domain is sufficient for the basic priming function of Munc13s since it can at least partially rescue the Munc13-deficient phenotype in neurons and chromaffin cells (Basu et al., 2005; Stevens et al., 2005). The binding of Munc18-1 to the N-terminus of Syntaxin-1 renders Syntaxin-1 in a closed conformation, which led to the hypothesis that the priming function performed by Munc13s is based on their ability to interact with Syntaxin-1. Munc13s could therefore induce a switch from the closed to an open Syntaxin-1 conformation, which would enable and/or accelerate SNARE complex nucleation (Ma et al., 2011, 2013; Sassa et al., 1999).

The C-terminus of Munc13 proteins possesses a single $\mathrm{C}_{2}\left(\mathrm{C}_{2} \mathrm{C}\right)$ domain, the function of which remains unknown (Brose et al., 1995; Shin et al., 2010). Further towards the Nterminus, the MUN domain is preceded by accessory domains that execute important regulatory roles for Munc13 function. Adjacent to the MUN domain, Munc13s contain a second $\mathrm{C}_{2}$ domain $\left(\mathrm{C}_{2} \mathrm{~B}\right)$ which is the only $\mathrm{C}_{2}$ domain of UNC-13/Munc13 proteins to bind phospholipids in a $\mathrm{Ca}^{2+}$-dependent manner, with a preference for phosphoinositides like phosphatidylinositol phosphate (PIP) and phosphatidylinositol 4,5-bisphosphat $\left(\mathrm{PIP}_{2}\right)$ (Shin et al., 2010). The $C_{2} B$ domain is preceded by a $C_{1}$ domain that is homologous to the diaglycerol (DAG)/phorbolester-binding domain of protein kinase C. Point mutations that disable the binding of DAG to Munc13-1 cause perinatal lethality in mice and a significant decrease in the RRP without changes in the EPSC amplitude, resulting in an increase in the vesicular release probability (Pvr) in autaptic neuron cultures (Ahmed et al., 1992; Aravamudan et al., 1999; Lackner, 1999; Maruyama and Brenner, 1991; Rhee et al., 2002). Preceeding the $C_{1}$ domain, members of the UNC-13/Munc13 proteins contain a Calmodulin-binding region, which is functionally highly conserved and mediates $\mathrm{Ca}^{2+}$ dependent Calmodulin-binding to UNC-13/Munc13 proteins (Aravamudan et al., 1999; Hu et al., 2013; Junge et al., 2004; Lipstein et al., 2012, 2013; Rodríguez-Castañeda et al., 2010). Mice which express a Munc13-1 variant that has lost its ability to bind $\mathrm{Ca}^{2+}$ Calmodulin, are viable, develop normally and show no major changes in basic synaptic transmission characteristics. However, these mice exhibited a deficit in synaptic vesicle priming during high activity at the calyx of Held synapse, a highly specialized 
glutamatergic synapse in the auditory pathway that is an established model to study plasticity characteristics (Lipstein et al., 2013).

In contrast to the highly conserved C-termini of UNC-13/Munc13 proteins, the $\mathrm{N}$-terminal domain structures vary significantly between isoforms. The only exceptions are UNC-13L in C.elegans and Munc13-1 and ubMunc13-2 in mammals, which exhibit homologous Nterminal sequences. These isoforms contain a third $C_{2}$ domain $\left(C_{2} A\right)$, which does not bind phospholipids in a $\mathrm{Ca}^{2+}$-dependent manner like the $\mathrm{C}_{2} \mathrm{~B}$ domain, but mediates binding to the Rab3a-interacting molecule (RIM), a cytoskeletal component of the presynaptic active zone that has been shown to have a role in localizing $\mathrm{Ca}^{2+}$-channels to the active zone and in docking and priming synaptic vesicles in their vicinity (Andrews-Zwilling et al., 2006; Betz et al., 2001; Fernández-Busnadiego et al., 2013; Han et al., 2011; Kaeser et al., 2011, 2012).

Mice lacking Munc13-1 die perinatally and electrophysiological recordings from glutamatergic hippocampal autaptic neurons in culture revealed a severe decrease in the RRP size measured by hyperosmotic sucrose solution, in the spontaneous release of synaptic vesicles and in $\mathrm{Ca}^{2+}$-dependent evoked release (Augustin et al., 1999a). Augustin et al., observed no changes in the number of synaptic vesicles and in the number of docked vesicles in electron micrographs after chemical fixation (Augustin et al., 1999a). Mice deficient of both Munc13-1 and Munc13-2 isoforms die prenatally and exhibit a complete shutdown of excitatory and inhibitory neurotransmission in hippocampal neuron cultures with no apparent changes in synapse density, synapse morphology, synaptic vesicle density and the number of membrane-attached synaptic vesicles in electron micrographs after chemical fixation (Varoqueaux et al., 2002).

Taken together, it is evident that members of the Munc13 family are essential proteins in neurotransmission since null mutants cause a complete loss of spontaneous and evoked synaptic transmission. The fact that application of hypertonic sucrose solution fails to trigger vesicle release in these mutants implies a complete absence of readily-releasable fusion-competent vesicles, despite normal numbers of morphologically docked synaptic vesicles in electron micrographs from chemically fixed samples. These findings established Munc13s as proteins important for mediating a molecular priming step downstream of synaptic vesicle docking. However, synapses lacking UNC-13 in C. elegans and Munc13-1 and -2 in mice were recently shown to exhibit an almost complete loss in the number of docked synaptic vesicles, analyzed using improved cryo-fixation methods combined with electron tomography, a method permitting three-dimensional reconstruction of synaptic organelles with nanometer resolution (See: 1.5.3 Transmission electron microscopy and electron tomography) (Gracheva et al., 2006; Siksou et al., 2009; 
Weimer et al., 2006). These findings indicated that Munc13s, major molecules in synaptic vesicle priming, also have a role in synaptic vesicle membrane-attachment and that the concept of subsequent docking and priming steps prior to synaptic vesicle fusion has to be revised.

\subsection{3. $\mathrm{Ca}^{2+}$-dependent activator protein for secretion (CAPS)}

The $\mathrm{Ca}^{2+}$-dependent activator protein for secretion (CAPS) family consists of two $\sim 145$ $\mathrm{kDa}$ proteins, which were originally identified as essential cytosolic factors for regulated $\mathrm{Ca}^{2+}$-dependent release in PC12 cells (Walent et al., 1992). CAPS proteins are highly conserved across species and have structural homology to members of the Munc13 family (Koch et al., 2000). Whereas $C$. elegans and Drosophila only express one CAPS isoform (UNC-31 \& dCAPS), it has been shown that mammals express two isoforms, CAPS-1 and -2 (Jockusch et al., 2007; Speidel et al., 2003). CAPS proteins contain a single Munc13 homology domain, which includes a Syntaxin-1-binding region (Betz et al., 1997; Khodthong et al., 2011; Koch et al., 2000). Moreover, the domain structure of CAPS proteins contains an $\mathrm{N}$-terminal dynactin-binding region, followed by $\mathrm{a}_{2}$ domain that can bind phospholipids in a $\mathrm{Ca}^{2+}$-dependent manner, indicating a role of CAPS in $\mathrm{Ca}^{2+}$ dependent membrane interactions (Grishanin et al., 2002; Sadakata et al., 2007a). The $\mathrm{C}_{2}$ domain is followed by a pleckstrin homology $(\mathrm{PH})$ domain, a structural motif that can associate with acidic phospholipids of the plasma membrane and that can bind to $\mathrm{PIP}_{2}$ (Grishanin et al., 2002). A C-terminal stretch of acidic amino acids mediates binding of CAPS to LDCVs and seems crucial for normal CAPS function in PC12 cell secretory vesicle release in addition to the required $\mathrm{Ca}^{2+}$-dependent $\mathrm{PIP}_{2}$ binding to the $\mathrm{PH}$ domain (Grishanin et al., 2002, 2004). For many years, most studies had indicated a specific role of CAPS in LDCV priming and release in neuroendocrine tissues (Elhamdani et al., 1999; Grishanin et al., 2002, 2004; Liu et al., 2008, 2010; Rupnik et al., 2000; Speidel et al., 2003, 2005, 2008; Tandon et al., 1998; Wassenberg and Martin, 2002).

Western blot analysis and immunotainings for CAPS- 1 reveals the presence of CAPS-1 in neuroendocrine tissues, for example in chromaffin cells of the adrenal medulla, in glucagon-containing a-cells of the pancreas, and in endocrine cells of the anterior pituitary (Sadakata et al., 2007b; Walent et al., 1992; Wassenberg and Martin, 2002). However, CAPS-1 is also strongly expressed in the brain, especially in the hippocampus and in the cerebellar granule cell layer, where its immunolabelling pattern co-localizes with the synaptic vesicle marker Synaptophysin, indicating a synaptic localization of the protein 
(Speidel et al., 2003; Walent et al., 1992; Wassenberg and Martin, 2002). CAPS-2 expression appears to be distinct from CAPS-1 and displays a strong level of colocalization with neutrophin-3 and brain-derived neurotrophic factor (BDNF), both factors important for neurodevelopmental processes in cerebellum (Sadakata et al., 2006, 2007c; Speidel et al., 2003). The widespread presence of CAPS in synaptic terminals in almost all brain regions, including terminals which do not contain many LDCVs, raised the question as to whether or not CAPS proteins have a role in synaptic vesicle release (Jockusch et al., 2007).

In Drosophila neuromuscular junction synapses, the loss of CAPS causes a severe reduction in LDCV release reflected by an accumulation of LDCVs in the terminals, but showed additional defects in the release of synaptic vesicles, with a reduction of the excitatory responses by $50 \%$ and a morphological accumulation of synaptic vesicles (Renden et al., 2001). However, the defects in glutamatergic synaptic vesicle release could not be rescued by genetically targeted expression of rat CAPS at the neuromuscular junction, leading to the interpretation that CAPS might influence fast synaptic transmission indirectly by preventing the delivery of components of the synaptic vesicle release machinery by LDCVs (Renden et al., 2001). Mice lacking both CAPS isoforms do not survive birth, whereas mice lacking only CAPS-2 are phenotypically indistinguishable at birth from wild-type mice (Jockusch et al., 2007; Speidel et al., 2003). Hippocampal autaptic neurons cultures from CAPS-1/2 double knock-out (DKO) mice display a complex phenotype. In $38 \%$ of all neurons, no evoked or spontaneous release could be detected, with a non-measurable RRP after hypertonic sucrose application (Jockusch et al., 2007). The remaining $62 \%$ of the cells exhibited a $68 \%$ decrease in the EPSC amplitude after stimulation and an $85 \%$ reduction in the size of the RRP. Synapse morphology and density as well as the number of total and docked synaptic vesicles in glutamatergic synapses were unchanged in electron micrographs of chemically fixed samples (Jockusch et al., 2007).

The current model poses that CAPS may prime synaptic vesicles by regulating SNARE complex assembly, since CAPS stimulates formation of trans-SNARE complexes from Syntaxin-1/SNAP25 acceptor and Synaptobrevin-2 donor in liposomes fusion assays (Daily et al., 2010; James et al., 2008, 2009, 2010; Khodthong et al., 2011). However, the role of CAPS proteins in synaptic vesicle priming is still heavily debated as many groups claim that the observed physiological deficits in neurotransmission in CAPS-1/2 DKO mice are rather an indirect consequence of deficits in synaptic LDCV secretion (reviewed in Rizo and Südhof, 2012; Stevens and Rettig, 2009; Südhof, 2012). Until now, the function 
of CAPS proteins in synaptic vesicle docking has not been assessed using improved cryopreservation methods for EM in any experimental setting.

\subsubsection{Synaptotagmin-1}

The fusion of docked and primed vesicles in response to action potential-evoked elevations in presynaptic $\mathrm{Ca}^{2+}$ concentrations occurs within milliseconds. The neuronal vesicular protein Synaptotagmin-1 has been proposed to be the $\mathrm{Ca}^{2+}$-sensor to trigger evoked release in neurons (Brose et al., 1992; Fernández-Chacón et al., 2001; Geppert et al., 1994).

Synaptotagmin-1 is a $65 \mathrm{kDa}$ vesicular protein comprising one $\mathrm{N}$-terminal TMR, which serves as a vesicular anchor, and two $\mathrm{C}_{2}$ domains, $\mathrm{C}_{2} \mathrm{~A}$ and $\mathrm{C}_{2} \mathrm{~B}$, which are connected by a flexible linker and able to bind three and two $\mathrm{Ca}^{2+}$ ions, respectively (Fernandez et al., 2001; Fernández-Chacón et al., 2001; Shao et al., 1998; Ubach et al., 1998). The $\mathrm{C}_{2}$ domains can bind anionic phospholipids (e.g. $\left.\mathrm{PIP}_{2}\right)$ in both a $\mathrm{Ca}^{2+}$-independent manner, through a stretch of polybasic amino acids, and in a $\mathrm{Ca}^{2+}$-dependent manner at the $\mathrm{Ca}^{2+}$ binding pocket (Araç et al., 2006; van den Bogaart et al., 2011; Li et al., 2006; Radhakrishnan et al., 2009). Moreover, Synaptotagmin-1 has been shown to interact with t-SNAREs and the SNARE complex (Choi et al., 2010; Kim et al., 2012; Lai et al., 2011; Rickman et al., 2004; Zhou et al., 2013). However, whether this interaction has physiological relevance for the $\mathrm{Ca}^{2+}$-triggering step in neurons in vivo remains unclear since Synaptotagmin-1 binding appeared to be weak and transient through electrostatic interactions, and may actually be indirect through Syntaxin-1-bound $\mathrm{PIP}_{2}$ (Choi et al., 2010; Honigmann et al., 2013).

Early studies showed that Synaptotagmin-1 KO mice die perinatally and that neurons lacking Synaptotagmin-1 exhibit a severe reduction in the fast component of the $\mathrm{Ca}^{2+}$ evoked EPSC. No changes were observed in the mEPSC frequency or in the size of the RRP after hypertonic sucrose application, a means of triggering release of primed vesicles in a $\mathrm{Ca}^{2+}$-independent manner (Geppert et al., 1994). Since then, many physiological functions have been proposed for Synaptotagmin-1 in addition to its ability to trigger $\mathrm{Ca}^{2+}$ dependent synchronous neurotransmitter release. However, different organisms or culture systems often revealed conflicting results. Synaptotagmin-1 was proposed to have a role in inhibiting release by acting as a fusion clamp that can be relieved in a $\mathrm{Ca}^{2+}$-dependent manner during SNARE-mediated fusion, reflected by an increase in the mini-frequency of inhibitory and excitatory PSCs in dissociated neuron cultures and hippocampal slice 
cultures from Synaptotagmin-1 KO mice (Kerr et al., 2008; Liu et al., 2009; Xu et al., 2009). Moreover, in neurons of C.elegans, Drosophila and mice, as well as in chromaffin cells, Synaptotagmin-1 deletion results in a reduction in the number of membraneattached synaptic vesicles and LDCVs, respectively, indicating a role of Synaptotagmins in a docking process prior to $\mathrm{Ca}^{2+}$-triggering (Jorgensen et al., 1995; Liu et al., 2009; Reist et al., 1998; de Wit et al., 2009; Yu et al., 2013). In addition, a reduction in total synaptic vesicle numbers was observed in Synaptotagmin-1 KO synapses, possibly related to a role of Synaptotagmin-1 in vesicle recycling (Jorgensen et al., 1995; Kononenko et al., 2013; Maritzen et al., 2010; Reist et al., 1998). In contrast to these morphological findings, cultured mammalian hippocampal neurons lacking Synaptotagmin-1 exhibit no, or only very little changes in the size of the RRP measured after application of hypertonic sucrose solution or during $\mathrm{Ca}^{2+}$-uncaging experiments (Burgalossi et al., 2012; Geppert et al., 1994; Liu et al., 2009; Xu et al., 2009). These results indicate that Synaptotagmin-1 most likely does not play an essential role in the physiological synaptic vesicle priming step, but rather support a model according to which Synaptotagmin-1 has, if any, a regulatory function in synaptic vesicle priming, in addition to its function as a $\mathrm{Ca}^{2+}$-sensor.

In summary, the mechanism by which Synaptotagmin-1 triggers $\mathrm{Ca}^{2+}$-evoked neurotransmitter release and the processes in which it participates prior to $\mathrm{Ca}^{2+}$-triggering (e.g. inhibitory fusion clamp for spontaneous release or membrane-attachment) remains unclear and a point of controversy (reviewed in Chapman, 2008; Jahn and Fasshauer, 2012; Rizo et al., 2006). The observed morphological docking phenotypes in Synaptotagmin-1 null mutant cells and results from recent in vitro studies indicate that the interaction of Synaptotagmin-1 with the plasma membrane and/or t-SNARE acceptor complexes might indeed be the molecular correlate of synaptic vesicle priming. This model challenges the idea of a (partially) assembled SNARE complex prior to fusion and would rather place SNARE complex zippering downstream of priming to result in immediate membrane fusion.

\subsubsection{Complexins}

Another protein family called Complexins has been shown to function in the $\mathrm{Ca}^{2+}$ triggering step of evoked neurotransmitter release in neurons (reviewed in Brose, 2008). In mammals, the Complexin family consists of four members (Complexin-1 to -4 ), each of around $20 \mathrm{kDa}$, with Complexin-1 and -2 being predominantly expressed in the brain and Complexin-3 and -4 being strongly expressed in the retina (Reim et al., 2001, 2005, 2009; Xue et al., 2008). Complexin-3 is also expressed to a lower extent in the brain (Xue et al., 
2008). Complexins are small molecules ( $\sim 20 \mathrm{kDa})$ that can bind with a central $\alpha$-helix in an antiparallel orientation to the at least partially assembled SNARE complex in a groove between the SNARE motifs of Synaptobrevin-2 and Syntaxin-1 (Chen et al., 2002). By this mode of action, murine Complexin isoforms have previously been shown to facilitate fast neurotransmitter release in a post-priming step ('super-priming') since the EPSC amplitude and the vesicular release probability was drastically reduced with no concomitant changes in the RRP size or in the number of docked vesicles in mouse hippocampal neurons (Reim et al., 2001; Xue et al., 2007, 2008). According to this model, Complexins might stabilize partially assembled SNARE complexes in the primed state prior to fusion, thus enabling rapid zippering after $\mathrm{Ca}^{2+}$-triggering. However, structurefunction analyses of murine and Drosophila Complexin isoforms revealed that distinct domains might execute different but conserved functions, with the central and an accessory $\alpha$-helix coordinating inhibitory effects of the fast, synchronous release and an unstructured N-terminal sequence facilitating $\mathrm{Ca}^{2+}$-evoked release (Cho et al., 2010; Xue et al., 2007, 2009, 2010). Recent studies described a drastic decrease in the RRP size and a massive increase in the mEPSC frequency after lentiviral knock-down of Complexins in mammalian neuron mass culture systems, which was not observed in similar knock-down experiments for Synaptotagmin-1, indicating a priming role for Complexins upstream of Synaptotagmin-1 function and an additional role as a fusion clamp for asynchronous and spontaneous release in concert with Synaptotagmin-1 (Cao et al., 2013; Kaeser-Woo et al., 2012; Tang et al., 2006; Yang et al., 2010). According to this model, Complexins would inhibit full SNARE complex zippering and therefore prevent synaptic vesicle fusion prior to $\mathrm{Ca}^{2+}$-triggering, suggesting a dominantly inhibitory role for Complexins. This model is supported by data obtained from C. elegans Complexin null mutants, which revealed a severe reduction in evoked release and in the number of docked vesicles, most likely due to an increase in spontaneous fusion events caused by an absence of inhibitory Complexin-mediated clamping-functions (Hobson et al., 2011). In Drosophila Complexin mutants, Complexin seems to regulate synchronous and asynchronous release with no changes in the number of membrane-attached synaptic vesicles at the neuromuscular junction synapse, thereby supporting the model of Complexins being inhibitors of spontaneous release and facilitators of evoked release, most likely by stabilizing partially assembled SNARE complexes and preventing premature synaptic vesicle fusion by preventing Synaptotagmin-1 / SNARE complex interactions (Jorquera et al., 2012).

In summary, the functional role of Complexins in mammalian neurons is still heavily debated. Most of the recent discussion has focused primarily on: (1) understanding the 
physiological relevance of a multiple role for Complexins in synaptic vesicle priming as well as in facilitating and inhibiting neurotransmitter release, and (2) resolving the molecular interactions of Complexins with the SNARE complex and with the $\mathrm{Ca}^{2+}$-sensor Synaptotagmin-1 (reviewed in Brose, 2008; Jahn and Fasshauer, 2012; Neher, 2010; Sørensen, 2009; Südhof and Rothman, 2009).

\subsection{Ultrastructural analysis of synaptic vesicle docking}

Transmission electron microscopy is the key method in studying synaptic morphology and the only method permitting the distances between synaptic vesicles and the active zone membrane to be measured accurately. EM enables the analysis of synaptic ultrastructure in the nanometer range, and due to the fact that plastic-embedded EM samples can be cut to sections as thin as $20 \mathrm{~nm}$, it has the highest z-resolution of any cellular microscopy techniques to date.

\subsubsection{Classical aldehyde-based fixation methods for electron microscopy}

Classically, EM studies have employed aldehyde-based (e.g. paraformaldehyde and glutaraldehyde) chemical fixation methods of the tissue followed by heavy metal membrane contrasting, dehydration and embedding in a plastic resin that allows ultrathin (20 - $100 \mathrm{~nm}$ ) sectioning (Hayat, 200; Sabatini et al., 1963). Aldehydes crosslink networks of proteins, thus preserving much of the ultrastructure of biological samples. In addition, the cell membranes are stained by heavy metals, such as osmium tetroxide $\left(\mathrm{OsO}_{4}\right)$, uranyl acetate or lead citrate, which readily react with phospholipids in lipid bilayers, resulting in an electron-dense precipitate on cell membranes for excellent contrast for EM (Hayat 2000). However, these traditional methods cause several problems when studying synaptic ultrastructure and in particular synaptic vesicle docking at the active zone. First, fixative diffusion into the tissue is a rather slow process (minutes to hours), which results in gradual rather than rapid fixation of the sample (Smith and Reese, 1980). Moreover, aldehydes have been shown to even trigger synaptic vesicle release during the fixation process, which makes reliable quantification of membrane-attached synaptic vesicles rather difficult (Smith and Reese, 1980). Second, the crosslinking process by aldehydes and heavy-metal stains result in dense protein networks composed of cytoskeletal components as it has been described for the presynaptic active zone ("dense projections", "presynaptic vesicular grid"), which appear as electron-dense, aggregated structures that 
may not provide a realistic picture of synaptic ultrastructure (Gray, 1963; Landis et al., 1988; Pfenninger et al., 1969, 1972; Südhof, 2012).

Third, the biggest problem for studying synaptic vesicle docking is the necessity for samples to be dehydrated prior to embedding in plastic, which cannot polymerize efficiently in the presence of water (Hayat 2000) This process, whereby sample tissue water is substituted by organic solvents (e.g. ethanol, propylene oxide), is typically performed at room temperature and introduces sample shrinkage and deformation of membranes that make the exact analysis of synaptic vesicle distances rather ambiguous (Möbius et al., 2010; Murk et al., 2003; Rostaing et al., 2006; reviewed in Verhage and Sørensen, 2008). To take these fixation artifacts into account, many studies defined all vesicles within a certain distance of the active zone membrane (e.g. a vesicle diameter of 30-40 nm) as docked. This definition enables the analysis of vesicle docking despite severe membrane deformations, but it clearly overestimates the number of membraneattached synaptic vesicles and cannot distinguish between loose tethering and actual membrane attachment.

\subsubsection{Cryo-fixation methods for electron microscopy}

In recent years, it has become obvious that rapid cryo-immobilization methods for EM possess several advantages in comparison to classical chemical fixation methods, and cryo-fixation has become the method of choice to study synaptic ultrastructure and dynamic cellular processes such as synaptic vesicle docking (Dubochet, 1995; Fernández-Busnadiego et al., 2010a, 2011, 2013; Frotscher et al., 2007; Heuser et al., 1979; Landis et al., 1988; Rostaing et al., 2006; Siksou et al., 2007, 2009; Zuber et al., 2005). Cryo-fixation methods exploit the ability of rapid freezing to prevent destructive icecrystal formation and promote the formation of vitreous (amorphous) ice. Samples thinner than $500 \mathrm{~nm}$ (e.g. monolayer neuron cultures, purified synaptosomes) can be frozen easily by a method called plunge freezing (Fernández-Busnadiego et al., 2011). Thicker samples (e.g. organotypic slice cultures, acute slices) require a method called highpressure freezing (reviewed in Dubochet 1995). In this process, the sample is frozen under high pressure ( 2100 bar) at low temperatures within milliseconds, resulting in vitrified samples that exhibit minimal perturbation of ultrastructure (Dubochet 2009). Highpressure freezing can rapidly $(<10 \mathrm{~ms}$ ) immobilize physiologically active synapses in cultured organotypic hippocampal slices from lethal KO mutants, providing snapshots of dynamic cellular processes, such as exo- and endocytosis of synaptic vesicles, to be captured with high temporal resolution (Fernández-Busnadiego et al., 2010; Frotscher et 
al., 2007; Siksou et al., 2009; Zuber et al., 2005). In contrast to the room temperature dehydration steps typically employed in classical EM sample preparation protocols, highpressure frozen samples are substituted at low temperatures to minimize sample shrinkage in a process called freeze substitution. During this process, cellular components are fixed and contrasted at subzero temperatures to minimize perturbation of ultrastructure prior to plastic embedding (Giddings, 2003; Jiménez et al., 2009; McDonald, 2007). Limitations of this method are that only relatively small samples are suitable for high-pressure freezing and that suboptimal cryo-fixation can result in artefacts (sometimes subtle) resulting from ice-crystal formation, such as deformed or ruptured membranes, that require careful evaluation of the ultrastructural preservation quality (Möbius et al., 2010). Moreover, the high-pressure applied during the freezing process requires the use of an external cryoprotectant / filler in order to prevent air in the freezing chamber and to improve ultrastructural sample preservation (McDonald, 2007). Commonly used cryoprotectants include dextran or sucrose solutions, which may increase the osmolarity of the sample environment to over 600 mOsm and may stimulate vesicular release from synaptic terminals (Dubochet, 1995; Fernández-Busnadiego et al., 2010; Südhof, 2012; Zuber et al., 2005).

As a consequence of these technical improvements, the optimized ultrastructural preservation for electron microscopy revealed a more accurate view of synaptic morphology in a near-native state. Reduced sample shrinkage, and therefore improved membrane preservation, enables the use of more stringent docking definitions, and usually exclusively vesicles in physical contact with the active zone membrane are considered docked (reviewed in Verhage \& Sørensen 2008). However, in 2D electron micrographs, vesicles will only appear in direct contact with the plasma membrane if their midline is contained within the volume of the ultrathin section, which will lead to an underestimation of the number of membrane-attached vesicles using this criterion. Moreover, curvature of the active zone membrane can obscure small gaps between synaptic vesicles and the plasma membrane, leading to the false-positive identification of non-attached vesicles. To circumvent this problem, electron tomography (ET), a method permitting three-dimensional reconstruction of synaptic organelles with nanometer resolution, can be applied.

\subsubsection{Transmission electron microscopy and electron tomography}

Transmission electron microscopy is a method in which a high voltage electron beam is transmitted through a semi-transparent, thin-sectioned specimen sample. The electrons 
are emitted by an electron gun, in which electrons originate from a cathode and are accelerated by high voltage $(\sim 80-300 \mathrm{kV})$ to provide enough energy to penetrate the specimen. The electron beam that passes the specimen conveys information about its structure and the image is magnified by a magnetic lens system within the microscope and finally detected by a charge-coupled device (CCD) camera. Cellular electron tomography is a technique, which employs a rotatable sample holder in order to allow acquisition of an image series at different tilt angles over a large angular range $\left(\sim-60^{\circ}\right.$ to $+60^{\circ}$, in $1-2^{\circ}$ increments). These two-dimensional projection images can be aligned (a process typically facilitated by application of surface fiducial markers), and then be used to reconstruct a three-dimensional volume, e.g. by applying a weighted backprojection algorithm (Koster et al., 1997). Due to technical limitations, the specimen cannot be tilted up to $90^{\circ}$ in one direction as the path length of electrons traversing the specimen increases dramatically at high angles which leads to increased electron scattering and induces noise and thus results in limitations in the sample thickness for imaging. In recent years (cryo)-electron tomographic techniques have revolutionized the field of neuroscience as they enable the capture of detailed synaptic structural and functional information like cytoskeletal arrangements, vesicle populations, exo-and endocytotic events, and most importantly provided the most reliable and reproducible tool to study synaptic vesicle docking to the active zone release sites (Fernández-Busnadiego et al., 2010, 2011, 2013; Liu et al., 2009; Siksou et al., 2007, 2009, 2011).

\subsection{Aim of the study}

The aim of the first part of the present study was to address the following questions:

(1) Do morphological synaptic vesicle docking and functional synaptic vesicle priming represent independent, subsequent steps prior to synaptic neurotransmitter release or are they indeed correlates of the same molecular process?

(2) Does synaptic vesicle docking require trans-SNARE interactions or is it rather mediated by Synaptotagmin-1 t-SNARE/plasma membrane binding.

The first question was primarily motivated by the fact that synapses lacking Munc13 priming proteins are completely devoid of fusion-competent as well as membraneattached synaptic vesicles in cryo-preserved samples for EM (Siksou et al., 2009). My fist aim was to set up a similar methodological approach combining the organotypic hippocampal slice culture system with high-pressure freezing fixation and freeze substitution for electron tomographic analysis. These methods would allow me to analyze synaptic parameters in an in-situ-like setting using lethal presynaptic mouse mutants that 
do not survive birth, and to apply high-resolution three-dimensional EM analysis to assess synaptic vesicle docking at glutamatergic, excitatory spine synapses of the hippocampus.

First, I studied members of the CAPS family, which have a crucial role in synaptic vesicle priming, but conventional chemical fixation methods for EM have not yet been able to reveal a deficit in synaptic vesicle membrane-attachment (Jockusch et al., 2007). I therefore planned to reassess the function of CAPS proteins in synaptic vesicle docking in my experimental setting.

Second, Munc13s and CAPS proteins are thought to initiate or regulate (partial) SNARE complex formation during vesicle priming. Therefore, my next aim was to elucidate the functions of the individual SNARE proteins Synaptobrevin-2, Syntaxin-1 and SNAP25 in synaptic vesicle docking. The outcome of these experiments would yield important information on the role of the SNARE complex in the synaptic vesicle docking/priming step. Moreover, it would help to answer the question whether stable trans-interactions between the v-SNARE Synaptobrevin-2 and the t-SNAREs Syntaxin-1 and SNAP25 would indeed be possible prior to $\mathrm{Ca}^{2+}$-triggering in vivo.

Third, I wanted to elucidate the function of the $\mathrm{Ca}^{2+}$-sensor Synyaptotagmin-1 in synaptic vesicle docking in my experimental system. Synaptotagmin-1 has recently been proposed as the vesicular molecular partner in the secretory vesicle membrane-attachment step (Liu et al., 2009; de Wit et al., 2009). If indeed the interaction of Synaptotagmin-1 with the tSNARE acceptor complex and/or the plasma membrane were the process underlying synaptic vesicle docking, Synaptobrevin-2 engagement and SNARE-complex formation would occur only downstream of synaptic vesicle docking during $\mathrm{Ca}^{2+}$-triggering and fusion. In this context I planned to reinvestigate the role of Complexins in synaptic vesicle docking in my experimental system. Complexin $\mathrm{KO}$ mice have been shown to exhibit a drastic decrease in evoked $\mathrm{Ca}^{2+}$-dependent release, with no changes in the size of the RRP or increase in the rate of spontaneous release. Other studies propose a role of Complexins in inhibiting full SNARE complex formation in concert with Synaptotagmin-1 prior to $\mathrm{Ca}^{2+}$-triggering and in synaptic vesicle priming.

The aim of the second part of the present study was to generate a Munc13-1 conditional $\mathrm{KO}$ mouse line. As mentioned above, genetic deletion of all Munc13 isoforms leads to complete shutdown of synaptic transmission in the majority of neuron types, with no effect on neuronal survival or major changes in neuron development and morphology in cultures. Loss of Munc13-1, the dominant isoform in most cell types, causes perinatal 
lethality, so that analysis of the function of Munc13-1 in neuronal networks in vivo has not yet been possible. A conditional Munc13-1 KO mouse line will help to understand the function of Munc13-1 in physiological networks, at different time-points of brain development and in brain regions that have been proven difficult to study in culture systems (e.g. Calyx of Held synapse). 


\section{Material and Methods}

\subsection{Animals}

All experiments performed on mice were conducted in compliance with the guidelines for the welfare of experimental animals issued by the Federal Government of Germany (Niedersächsisches Landesamt für Verbraucherschutz und Lebensmittelsicherheit Permission 33.9.42502-04/103/08) and the Max Planck Society. The mouse lines used in this study and a reference to the original publication are listed below. Munc13, CAPS and Complexin knock-out (KO) mouse lines were originally generated in the host laboratory of Prof. Nils Brose. A Munc13-1 conditional knockout mouse line was generated within this study and is not listed below.

\subsubsection{Mouse lines}

Munc13-1 KO (Augustin et al., 1999a)

Munc13-2 KO (Varoqueaux et al., 2002)

CAPS-1 KO (Speidel et al., 2005)

CAPS-2 KO (Jockusch et al., 2007)

Complexin-1 KO (Reim et al., 2001)

Complexin-2 KO (Reim et al., 2001)

Complexin-3 KO (Xue et al., 2008)

Synaptotagmin-1 KO (Geppert et al., 1994), bought from "The Jackson Laboratory"

Synaptobrevin-2 KO (Schoch et al., 2001), bought from "The Jackson Laboratory"

SNAP25 KO (Washbourne et al., 2002), kindly provided by Dr. M. Loos and Dr. I. Herfort

Syntaxin-1A KO (Gerber et al., 2008), kindly provided by Prof. C. Rosenmund

Syntaxin-1B ${ }^{\text {YFP }}$ (Arancillo et al., 2013), kindly provided by Prof. T. Südhof and Prof. C. Rosenmund

\subsection{Molecular Biology}

\subsubsection{Materials}

\subsubsection{Chemicals and Reagents}

\begin{tabular}{|l|l|l|}
\hline Product & Company & Cat. No. \\
\hline $1 \mathrm{~kb}$ DNA ladder, $0.05 \mu \mathrm{g} / \mu \mathrm{l}$ & Fermentas & SM0311 \\
\hline $10 \times$ RedTaq® PCR Reaction Buffer & Sigma-Aldrich & B5926 \\
\hline $100 \mathrm{bp}$ DNA ladder, $0.05 \mu \mathrm{g} / \mu \mathrm{l}$ & Fermentas & SM0322 \\
\hline
\end{tabular}




\begin{tabular}{|c|c|c|}
\hline Product & Company & Cat. No. \\
\hline 10x Cloned Pfu DNA Polymerase Buffer & Stratagene & 200532 \\
\hline 10x rAPid Alkaline Phosphatase Buffer & Roche & 04898133001 \\
\hline $1 \mathrm{~kb}$ DNA ladder & Invitrogen & $15615-016$ \\
\hline $1 \mathrm{~kb}$ DNA ladder $0.1 \mu \mathrm{g} / \mu \mathrm{l}$ & Gibco / Invitrogen & $15615-016$ \\
\hline $\begin{array}{l}\text { 5-Bromo-4-chloro-3-indolyl- beta-D-galactopyranoside } \\
\text { (X-Gal) }\end{array}$ & Biomol & 0.2249 \\
\hline 5x Ligation Buffer & Invitrogen & $46300-018$ \\
\hline Ammonium Acetate $\left(\mathrm{NH}_{4} \mathrm{Ac}\right)$ & Merck & 1.011 .161 .000 \\
\hline Ampicillin & Sigma-Aldrich & \\
\hline BD Bacto ${ }^{\mathrm{TM}}$ Agar & BD Diagnostic Systems & 214010 \\
\hline Bromophenol Blue & Merck & 8122 \\
\hline BSA (digest) & New England Bio Labs & B90015 \\
\hline Chloramphenicol & Calbiochem & 220551 \\
\hline Chloroform & Merck & 1.024 .451 .000 \\
\hline Cloned Pfu Polymerase & Stratagene & $600153-81$ \\
\hline dNTP Set & Bioline & $\mathrm{BIO}-39025$ \\
\hline Ethylenediaminetetraacetic Acid Trisodium Salt (EDTA) & Sigma & E1644 \\
\hline Ethanol & Sigma-Aldrich & 32205 \\
\hline Etidium Bromide & Carl Roth & 2218.2 \\
\hline Gel Red & BIOTIUM & $730-2958$ \\
\hline Glycerol & Merck & 1.040 .940 .500 \\
\hline Hydrochloric Acid (HCL) & Merck & 1.003 .172 .500 \\
\hline Isopropanol & Merck & 1.096 .342 .500 \\
\hline Isopropyl $\beta$-D-1-thiogalactopyranoside (IPTG) & Carl Roth & 2316.4 \\
\hline Kanamycin & Sigma-Aldrich & \\
\hline L-(+)-arabinose & Sigma-Aldrich & A3256 \\
\hline Luria Broth & Sigma-Aldrich & L3522 \\
\hline Lysozym & Sigma-Aldrich & L6876 \\
\hline MidRange PFG Marker I & New England Bio Labs & N3551S \\
\hline NEB buffers & New England Bio Labs & \\
\hline P1 buffer & Quiagen & 19051 \\
\hline P2 buffer & Quiagen & 19052 \\
\hline P3 buffer & Quiagen & 19053 \\
\hline Phenol & Carl Roth & 382 \\
\hline Plastikwaren & $\begin{array}{l}\text { Greiner, Falcon, Costar und } \\
\text { Brandt }\end{array}$ & \\
\hline Potassium chloride (KCl) & Merck & 1.049 .361 .000 \\
\hline rAPid Alkaline Phosphatase & Roche & 04898133001 \\
\hline REDTaq® DNA Polymerase & Sigma-Aldrich & D4309 \\
\hline Restriction Enzymes & New England Bio Labs & \\
\hline Rnase A & Roche & $\begin{array}{l}10109169001 / 11 \\
3\end{array}$ \\
\hline Sodium Acetate (NaAc) & Merck & 1.062 .680 .250 \\
\hline Sodium Chloride ( $\mathrm{NaCl})$ & Merck & 1.076 .511 .000 \\
\hline Tris-Base & Sigma-Aldrich & T1503 \\
\hline
\end{tabular}




\begin{tabular}{|l|l|l|}
\hline Product & Company & Cat. No. \\
\hline T4 DNA ligase & Invitrogen & $15224-017$ \\
\hline Triton-X-100 & Roche & 10789704001 \\
\hline UltraPure ${ }^{\text {TM }}$ Agarose & Invitrogen & 16500500 \\
\hline Lysozym & Sigma-Aldrich & L6876 \\
\hline MidRange PFG Marker I & New England Bio Labs & N3551S \\
\hline
\end{tabular}

\subsubsection{Equipment}

\begin{tabular}{|l|l|}
\hline Product & Company \\
\hline BIO-RAD electroporator & BIO-RAD \\
\hline BioScience Ultrospec3100pro & Amersham \\
\hline Gene Amp PCR system & Applied Biosystems \\
\hline Peltier Thermal Cycler 255 & MJR Research \\
\hline Chef-DR II Pulsed Field Electrophoresis System & Bio-RAD \\
\hline
\end{tabular}

\subsubsection{Media and buffer}

Ampicillin Stock $20 \mathrm{mg} / \mathrm{ml}$

Kanamycin Stock $10 \mathrm{mg} / \mathrm{ml}$

Chloramphenicol Stock $25 \mathrm{mg} / \mathrm{ml}$ in ethanol

LB-Medium ( $25 \mathrm{~g}$ Luria Broth in $1 \mathrm{~L} \mathrm{ddH}_{2} \mathrm{O}$ )

LB Agar plates (15 g Bacto-Agar in $1 \mathrm{~L}$ LB Medium

6x Loading Buffer $(10 \mathrm{ml} 1 \mathrm{M}$ Tris- $\mathrm{HCl} \mathrm{pH}$ 7.5, $300 \mathrm{mg}$ Bromphenolblau, $600 \mathrm{ml} 87 \%$ Glycerol, 120 ml 0.5 M EDTA; add $\mathrm{H}_{2} \mathrm{O}$ to $\left.1000 \mathrm{ml}\right)$

STET buffer (100 mM NaCl, $10 \mathrm{mM}$ Tris/HCl (pH 8.0), $1 \mathrm{mM}$ EDTA, 5\% Triton X-100)

TE buffer (10 mM Tris/HCl (pH 7.4), 1 mM EDTA)

1 XTBE buffer was used ( $89 \mathrm{mM}$ Tris, $8 \mathrm{mM}$ boric acid, $0.2 \mathrm{mM} \mathrm{Na} \mathrm{a}_{2}$-EDTA)

1xTAE buffer ( $40 \mathrm{mM}$ Tris, $20 \mathrm{mM}$ acetic acid, and $1 \mathrm{mM}$ EDTA)

P1 buffer (50 mM TrisCl (pH 8.0), 10 mM EDTA, $100 \mu \mathrm{g} / \mathrm{ml}$ RNase A)

P2 buffer (200 mM NaOH, 1\% SDS (w/v))

P3 buffer (3.0 M potassium acetate ( $\mathrm{pH} 5.5)$

Lysozyme solution $(10 \mathrm{mg} / \mathrm{ml}$ in STET buffer)

\subsubsection{Vector plasmids}

\begin{tabular}{|l|l|}
\hline Product & Company/Institution \\
\hline pBluescript II SK +/- & Stratagene, Heidelberg \\
\hline PL253 & Biological Resources Branch, DCTD \\
& NCl-Frederick Cancer Research and \\
& Development Center \\
\hline
\end{tabular}




\begin{tabular}{|l|l|}
\hline Product & Company/lnstitution \\
\hline PL452 & $\begin{array}{l}\text { Biological Resources Branch, DCTD } \\
\text { NCl-Frederick Cancer Research and } \\
\text { Development Center }\end{array}$ \\
\hline PL451 & $\begin{array}{l}\text { Biological Resources Branch, DCTD } \\
\text { NCl-Frederick Cancer Research and } \\
\text { Development Center }\end{array}$ \\
\hline pCR 2.1-TOPO® vector & Invitrogen \\
\hline bMQ 441 I13 (pBACe3.6) & The Welcome Trust Sanger Institute \\
\hline pFUGW & Salk Inst., Dr. Inder M. Verma \\
\hline pFUGW-iCre & $\begin{array}{l}\text { Provided by Prof. Dr. Richard L. Huganir (Lois et } \\
\text { al., 2002) }\end{array}$ \\
\hline PACK & Provided by Prof. Dr. Pavel Osten \\
\hline ENV & Provided by Prof. Dr. Pavel Osten \\
\hline &
\end{tabular}

The pFUGWiCre vector with an insertion of IRES-iCre downstream of EGFP in the pFUGW vector (Lois et al., 2002) was a kind gift from Dr. R. Huganir (Baltimore, MD, USA).

\subsubsection{Bacterial Strains}

\begin{tabular}{|l|l|}
\hline Product & Company \\
\hline E. coli competent XL1-Blue cells & Stratagene, Heidelberg \\
\hline E.coli competent Electro10-Blue & Stratagene, Heidelberg \\
\hline EL350 & $\begin{array}{l}\text { Biological Resources Branch, DCTD } \\
\text { NCl-Frederick Cancer Research and } \\
\text { Development Center }\end{array}$ \\
\hline \multirow{2}{*}{ SW106 } & $\begin{array}{l}\text { Biological Resources Branch, DCTD } \\
\text { NCl-Frederick Cancer Research and } \\
\text { Development Center }\end{array}$ \\
\hline
\end{tabular}

\subsubsection{Kits}

\begin{tabular}{|l|l|l|}
\hline Product & Company & Cat. No. \\
\hline EndoFree Plasmid Maxi Kit & Quiagen & 12362 \\
\hline Nexttec & $\begin{array}{l}\text { nexttec } \\
\text { GmbH Biotechnologie }\end{array}$ & 10.924 \\
\hline PureLink ${ }^{\text {TM }}$ HiPure Plasmid Filter Midiprep Kit & Invitrogen & K2100-14 \\
\hline PureLink $^{\text {TM }}$ PCR Purification Kit & Invitrogen & K3100-02 \\
\hline PureLink ${ }^{\text {TM }}$ Quick Plasmid Miniprep Kit & Invitrogen & K2100-11 \\
\hline QIAquick Gel Extraction Kit & Quiagen & 28706 \\
\hline QIAquick PCR Purification Kit & Quiagen & 28106 \\
\hline TOPO TA Cloning® Kit & Invitrogen & K4510-20 \\
\hline
\end{tabular}




\subsubsection{Oligonucleotides}

All oligonucleotides used in this study were synthesized in the DNA Core Facility of the Max-Planck Institute of Experimental Medicine. Oligonucleotide sequences are listed below with their Lab ID number. Restriction sites used for molecular cloning are indicated. The primers 27121 - 27124 were used for the genotyping of the Munc13-1 CKO mouse line and will be abbreviated in the following to \#1 - \#4.

\begin{tabular}{|c|c|c|}
\hline Primer ID & Sequence & \begin{tabular}{|l} 
Restr. \\
Site
\end{tabular} \\
\hline 8431 & 5'-AGAGCCAAAGCCAACTG-3' & \\
\hline 8432 & 5'-GACGTCCAACTTCACCTGT-3' & \\
\hline 19929 & 5'-GAGGTACCCGCATACAGCTGGCTCCTTCATCA-3' & Kpnl \\
\hline 19930 & 5'-CCGAATTCAAGCTTGTCTCCCCATCCACCAACCACTT-3' & $\begin{array}{l}\text { EcoRI, } \\
\text { HindIII }\end{array}$ \\
\hline 19931 & 5'-GGGGATCCAATTGGTAGGTGGACAGACAGATG-3' & BamHI \\
\hline 19932 & 5'-AAGCGGCCGCTCCCTCACGTCATGGCTTGGTAGA-3' & Notl \\
\hline 19965 & 5'-TCETCGAGGTGACGGACGTGCAGAACAATGGTG-3' & Xhol \\
\hline 19966 & 5'-CAGAATTCATGTAGCCAAGGATGGTCCTGTGCTCCAGATC-3' & EcoRI \\
\hline 19967 & 5'-GGGGATCCACTGGGTGGGAAACCATCGGGCTACAA-3' & $\mathrm{BamHI}$ \\
\hline 19968 & 5'-GACCGCGGGAAACACTGGGCAGAGGCACACG-3' & Sacll \\
\hline 21137 & 5'-GAGTGCGGCCGCTGATGTCAAGTGTTCAGAATCGATG-3' & Notl \\
\hline 21138 & 5'-GAAAAAGCTTCCAAAAATAAATAATAAATAAATAACAACAAACTA-3' & HindIII \\
\hline 21139 & 5'-TGCAAAAAGCTTCAACTGTCATGTGGGTGATGGAAATC-3' & HindIII \\
\hline 21140 & 5'-CAGGGGGATCCTGAGAAGTGGGGTATGATGGT-3' & BamHI \\
\hline 21141 & 5'-TGCTGAGGTGATGTGGAGCCTATT-3' & \\
\hline 21142 & 5'-AGGTCTTGGTGACATCAGGACTTA-3' & \\
\hline 24607 & 5'-GCTGGGGATGCGGTGGGCTCTA-3' & \\
\hline 24608 & 5'-TGTGCGTAGTAGGCGTTGATGTTG-3' & \\
\hline 24609 & 5'-CCATCCGGCTTCACATCAGT-3' & \\
\hline 24610 & 5'-AGGCCTACCCGCTTCCATTG-3' & \\
\hline 25153 & 5'-CATCGCATTGTCTGAGTAGGTGTC-3' & \\
\hline 25154 & 5'-GGGTTTAGGGAGTTTGTTTTTAGA-3' & \\
\hline 25155 & 5'-GTGTGGCTAAGGACGAGTGT-3' & \\
\hline $27121(\# 1)$ & 5'-CTCTATGGCTTCTGAGGCGGAAA-3' & \\
\hline $27122(\# 2)$ & 5'-AGTTTTCATCTTGTAGCCCGAT-3' & \\
\hline $27123(\# 3)$ & 5'-CAACTGGCCAAGAACTAGAGGA-3' & \\
\hline $27124(\# 4)$ & 5'-GCACGGAATGTTGAATGGTCTT -3' & \\
\hline
\end{tabular}




\subsubsection{Methods}

\subsubsection{Generation of electrocompetent E. coli cells}

A single colony was picked and transferred to $5 \mathrm{ml}$ LB to grow for $16-18 \mathrm{~h}$ with shaking. Three $\mathrm{ml}$ of the culture was transferred to $60 \mathrm{ml}$ of LB (1:20 dilution) and incubated to reach an optical density measured at $600 \mathrm{~nm}$ wavelength $\left(\mathrm{OD}_{600}\right)$ of $0.5-0.6$. Cells were immediately put into ice slush and shaken for $5 \mathrm{~min}$ for a fast temperature drop and then placed on ice for additional $10 \mathrm{~min}$. Cells were collected at $3,500 \mathrm{rpm}$ at $0^{\circ} \mathrm{C}$ for $10 \mathrm{~min}$ and washed three times with ice-cold water. Cells were resuspended in $400 \mu$ sterile icecold water and $50 \mu \mathrm{l}$ aliquots were prepared in precooled Eppendorf tubes and stored on ice. For long-term storage of cells, cells were washed and resuspended with $15 \%$ glycerol in water. Aliquots were immediately frozen in liquid nitrogen and stored at $-80^{\circ} \mathrm{C}$.

\subsubsection{Inducing Recombineering Enzymes}

EL350 or SW106 cells grew in $5 \mathrm{ml}$ LB at $32{ }^{\circ} \mathrm{C}$ for $16-18 \mathrm{~h}$ with shaking. $3 \mathrm{ml}$ of the culture was transferred to $60 \mathrm{ml}$ of LB (1:20 dilution) and incubated to reach an $\mathrm{OD}_{600}$ of $0.5-0.6 .50 \mathrm{ml}$ of the cells were split into two new flasks and shaken in a $42^{\circ} \mathrm{C}$ water bath for $15 \mathrm{~min}$. The cells were immediately put into ice slush and shaken for $5 \mathrm{~min}$ and then placed on ice for additional $10 \mathrm{~min}$. Cells were then prepared for deoxyribonucleic acid (DNA) plasmid electroporation.

\subsubsection{Excision of the Neo cassette by arabinose induced Cre expression}

EL350 or SW106 cells were grown in $5 \mathrm{ml} \mathrm{LB}$ at $32^{\circ} \mathrm{C}$ for $16-18 \mathrm{~h}$ with shaking. The $5 \mathrm{ml}$ culture was then added to $250 \mathrm{ml} \mathrm{LB}$ to grow at $32^{\circ} \mathrm{C}$ to reach an $\mathrm{OD}_{600}$ of 0.4 . $\mathrm{A} 10 \%$ solution of $\mathrm{L}-(+)$-arabinose in $\mathrm{ddH}_{2} \mathrm{O}$ was added to reach a final concentration of $0.1 \%$ and cells were shaken for another hour. Cells were then prepared for DNA plasmid electroporation. 


\subsubsection{Transformation of bacterial strains}

Electroporation of bacterial artificial chromosome (BAC) or plasmid DNA into bacterial strains was performed using a BIO-RAD electroporator at $1.8 \mathrm{kV}, 25 \mu \mathrm{F}$ with pulse controller set at $200 \Omega$. For conventional and $\mathrm{pCR} \otimes 2.1-\mathrm{TOPO} \circledast$ ligation reactions, 1 - $2 \mu \mathrm{l}$ of the sample was electroporated per $50 \mu$ lectrocompetent cells. For freshly purified BAC DNA, $1 \mu \mathrm{l}(\approx 4 \mu \mathrm{g})$ was electroporated. Then, $1 \mathrm{ml}$ LB was added to each cuvette, cells were transferred to Eppendorf or $14 \mathrm{ml}$ Falcon tubes and incubated at $37^{\circ} \mathrm{C}$ for $1 \mathrm{~h}$. Cells were then spread in different concentrations on LB agar plates containing appropriate antibiotics for selection. For plasmid retransformations, typically only $1 \mathrm{ng}$ DNA was electroporated. Cells were not recovered, but $20 \mu \mathrm{l}$ was plated immediately onto LB agar plates containing appropriate antibiotics for selection.

\subsubsection{Glycerol stocks}

$750 \mu \mathrm{l}$ from an overnight culture of transformed E. coli was mixed with $750 \mu \mathrm{l} 30 \%$ glycerol in LB in a cryo-tube, incubated for $3 \mathrm{~min}$ at room temperature and stored at $-80^{\circ} \mathrm{C}$. To plate cells from a glycerol stock, the tube was transported to the bench on dry ice and cells were spread and isolated with a sterile inoculating loop.

\subsubsection{BAC DNA preparation}

E. coli cells with BACs were grown in $5 \mathrm{ml}$ of LB containing chloramphenicol for 16-18 $\mathrm{h}$. Cells were collected at $3500 \mathrm{rpm}$ for $15 \mathrm{~min}$ and resuspended in $250 \mu \mathrm{l}$ buffer P1 . Cells were lysed in $250 \mu \mathrm{l}$ buffer $\mathrm{P} 2$ for $5 \mathrm{~min}$ at room temperature and the sample neutralized by buffer P3 . Tubes were spun for $4 \mathrm{~min}$ at $11,000 \mathrm{rpm}$. The supernatant was transferred to a new tube and spun again for $4 \mathrm{~min}$ at $11,000 \mathrm{rpm}$. The supernatant was transferred to a new tube and BAC DNA was precipitated for $10 \mathrm{~min}$ at room temperature by adding 750 $\mu \mathrm{l}$ isopropanol. The DNA pellet was collected at $15,000 \mathrm{rpm}$ for $10 \mathrm{~min}$, washed once with $1 \mathrm{ml} 70 \%$ ethanol, dried for $3 \mathrm{~min}$ at room temperature and finally resuspended in $50 \mu \mathrm{l}$ TE buffer. 


\subsubsection{Plasmid DNA preparation}

To purify plasmid DNA from bacterial cultures for sequencing, the PureLink ${ }^{\text {TM }}$ Quick Plasmid Miniprep Kit was used. In order to purify high yields of plasmid DNA from bacterial cultures, the PureLink ${ }^{\mathrm{TM}}$ HiPure Plasmid Filter Midiprep Kit was used. To optain high yields of pure, endotoxin-free plasmid DNA for ES cell electroporation and HEK293FT cell transfection, the EndoFree Plasmid Maxi Kit was used. For detailed protocols for these Kits, please refer to the product manuals.

\subsubsection{Agarose gel electrophoresis and Pulsed field gel electrophoresis}

Agarose gel electrophoresis is a technique, which allows separating DNA based on its size in an electric field. The distance between DNA bands of different sizes depends on the percent agarose in the gel. Usually $0.7-1.5 \%$ agarose gels were used and the gels run at a constant voltage of 80-120 V. Samples were loaded in 1x loading buffer. For

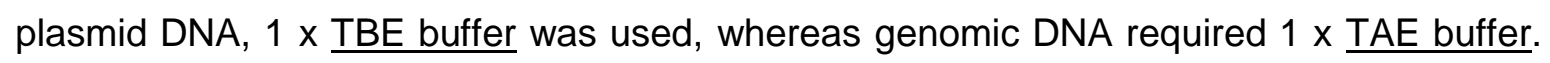
To visualize DNA in the gel, ethidium bromide, a DNA intercalator, was added in a concentration of $0.5 \mu \mathrm{g} / \mathrm{ml}$ to the agarose gel. Alternatively, to visualize DNA fragments for genotyping purposes, GelRed, a nucleic acid stain, was added the agarose gel in a 1:200,000 dilution. Both substances, ethidium bromide and GelRed, fluoresce under ultraviolet light. DNA fragment sizes were estimated by different ladder mixes run in parallel (100 bp DNA ladder (Fermentas), 1 kb DNA ladder (Fermentas, Invitrogen)).

Pulsed field gel electrophoresis was used for the separation of large DNA molecules (i.e. BAC DNA fragments) by applying an electric field that periodically changes direction. In order to analyze the BAC clones by restriction enzyme digest, DNA samples were

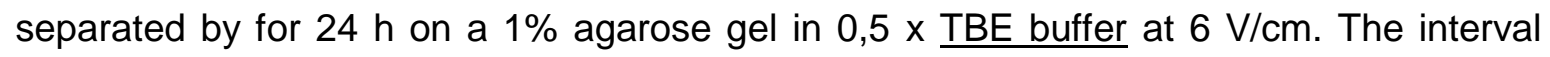
length was changing from an initial $1 \mathrm{sec}$ to a final $25 \mathrm{sec}$ in order to separate all fragments. The MidRange PFG Marker I (New England Bio Labs) ladder was run in parallel in order to estimate the DNA fragment sizes. 


\subsubsection{Agarose gel extraction of DNA fragments}

DNA fragments were purified with the QIAquick Gel Extraction Kit. For a detailed protocol for this Kit, please refer to the product manual.

\subsubsection{Phenol/Chloroform Extraction for large ( $\geq 10 \mathrm{~kb}$ ) DNA fragments}

The linearized Munc 13-1 cko targeting vector was extracted by Phenol/Chloroform extraction. In a first step Phenol/Chloroform (1:1) was added to the digestion mix and vortexed for $1 \mathrm{~min}$. Samples were centrifuged at 15,000 x g for $15 \mathrm{~min}$ and the upper phases were transferred into fresh tube. This procedure was repeated three times. After the last step, $300 \mu$ chloroform was added to the upper phases, vortexed again for $1 \mathrm{~min}$ and the samples were centrifuged at $15,000 \times \mathrm{g}$ for $15 \mathrm{~min}$. All following steps were performed in a cell culture hood. The upper phases were transferred into sterile Eppendorf tubes. To precipitate the DNA, $2.5 \mu \mathrm{l} 3 \mathrm{M} \mathrm{NaAc}(\mathrm{pH} 5.2)$ and $500 \mu \mathrm{l} 100 \%$ ethanol were added and the samples stored at $-20^{\circ} \mathrm{C}$ overnight. The next day, samples were centrifuged at $15,000 \times \mathrm{g}$ for $15 \mathrm{~min}$, washed three times with $70 \%$ ethanol, pooled, airdried and resuspended in $200 \mu$ sterile-filtered $0.1 \times$ TE.

\subsubsection{Polymerase Chain Reaction}

The polymerase chain reaction (PCR) is a method to amplify specific DNA sequences in order to obtain a large number of copies. The main steps of the reaction are a denaturation step, in which the double-stranded DNA template melts into its single strand, an annealing step, in which short oligonucleotides (primers) can anneal to the DNA strands, and an elongation step, in which the DNA polymerase synthesizes a new DNA strand complementary to the DNA template. The duration and the temperature of the individual steps depend highly on the DNA polymerase used as well as on the melting temperatures of the individual primer pairs used. To check the PCR results, $1 \mu$ of each sample was loaded onto an agarose gel.

PCR amplifications in order to produce PCR products used for subsequent cloning reactions were done using the Cloned Pfu Polymerase with a proof reading function and were performed in the following reaction mix using a Gene Amp PCR system 2400 with varying annealing temperatures $(\mathbf{X})$ : 


\begin{tabular}{|l|l|}
\hline PCR Mix & PCR Settings \\
\hline $1 \mu \mathrm{l}-$ BAC DNA (10ng) & $95^{\circ} \mathrm{C}-1$ min \\
$2,5 \mu \mathrm{l}-$ primer $1(12,5 \mathrm{pM})$ & 30 cycles of: \\
$2,5 \mu \mathrm{l}-$ primer 2 $(12,5 \mathrm{pM})$ & $95^{\circ} \mathrm{C}-45 \mathrm{sec}$ \\
$5 \mu \mathrm{l}-10 \mathrm{x}$ Pfu buffer & $\mathbf{X}^{\circ} \mathrm{C}-45 \mathrm{sec}$ \\
$4 \mu \mathrm{l}-$ dNTPs & $72^{\circ} \mathrm{C}-60 \mathrm{sec}$ \\
$1 \mu \mathrm{l}-$ Pfu Polymerase & followed by: \\
$34 \mu \mathrm{l}-\mathrm{ddH}_{2} \mathrm{O}$ & $72^{\circ} \mathrm{C}-10$ min \\
\hline
\end{tabular}

PCR products were purified either with PureLink ${ }^{\mathrm{TM}}$ PCR Purification Kit or the QIAquick PCR Purification Kit. For detailed protocols for these Kits, please refer to the product manuals.

PCR amplifications for quick genotyping PCRs or PCR screens were performed using a Red Taq DNA Polymerase in following reaction mix using a Peltier Thermal Cycler 255 with varying annealing temperatures $(\mathbf{X})$ :

\begin{tabular}{|l|l|}
\hline PCR Mix & PCR Settings \\
\hline $1 \mu \mathrm{l}-$ bacterial culture / DNA & $94^{\circ} \mathrm{C}-3$ min \\
$0.1 \mu \mathrm{l}-$ primer $1(0.5 \mathrm{pM})$ & 30 cycles of: \\
$0.1 \mu \mathrm{l}-$ primer $2(0.5 \mathrm{pM})$ & $94^{\circ} \mathrm{C}-30 \mathrm{sec}$ \\
$2 \mu \mathrm{l}-10 \mathrm{x}$ Red Taq Buffer & $\mathbf{X}^{\circ} \mathrm{C}-30 \mathrm{sec}$ \\
$1 \mu \mathrm{l}-$ dNTPs & $72^{\circ} \mathrm{C}-60 \mathrm{sec}$ \\
$1 \mu \mathrm{l}-$ Red Taq Polymerase & followed by: \\
$15,8 \mu \mathrm{l}-\mathrm{ddH}_{2} \mathrm{O}$ & $72^{\circ} \mathrm{C}-7$ min \\
\hline
\end{tabular}

\subsubsection{Dephosphorylation of 5'DNA ends:}

The 5' ends of vector DNA were dephosphorylated by incubating $1 \mu \mathrm{l}(1 \mathrm{U})$ rAPid alkaline phosphatase and $2 \mu \mathrm{l} 10 \mathrm{x}$ rAPid alkaline phosphatase buffer with $17 \mu \mathrm{l}$ DNA for $10 \mathrm{~min}$ at $37^{\circ} \mathrm{C}$ and subsequent inactivation of the enzyme for 2 min at $75^{\circ} \mathrm{C}$.

\subsubsection{DNA Ligation and subcloning in TOPO $\mathrm{pCR}$ vectors}

The ligation reaction mix was prepared in a 1:3 ratio of dephosphorylated vector DNA to insert DNA and in a total volume of $14 \mu \mathrm{l} .4 \mu \mathrm{l}$ of $5 \mathrm{x}$ ligation Buffer and $2 \mu \mathrm{l}$ of T4 DNA ligase were added and incubated overnight in a cold water bath. $1 \mu \mathrm{l}$ of the ligation mix was electroporated into Electro10 Blue or XL1-Blue cells and spread onto LB agar plates 
containing appropriate antibiotics for selection. TOPO TA Cloning® requires Taq polymerase amplified PCR products. Taq polymerase has a terminal transferase activity that adds a deoxyadenosine to the 3 ' end of a PCR fragment. The TOPO TA Cloning ${ }^{\circledR}$ Kit provides a linearized $\mathrm{PCR} \otimes 2.1-\mathrm{TOPO} \otimes$ vector, which has a 3' deoxythymidine overhang that allows efficient ligation of PCR product and vector, and has the cloning site in the lacZ gene, which encodes for the B-galactosidase for selection. For a detailed protocol for this Kit, please refer to the product manual. Briefly, 1-2 $\mu$ l of the TOPO reaction was electroporated in Electro10 Blue cells and spread onto LB agar plates containing ampicillin, IPTG and X-Gal for selection.

\subsubsection{Dirty Mini Prep and test digest. (Holmes and Quigley, 1981)}

In order to test plasmids from a large number of transformed bacterial clones by restriction enzyme test digests, cells from $2 \mathrm{ml}$ overnight bacterial culture were harvested at 15,000 $\mathrm{x}$ $\mathrm{g}$ for $1 \mathrm{~min}$. The pellet was then resuspended in $300 \mu \mathrm{l}$ STET buffer. $25 \mu \mathrm{l}$ of lysozyme solution was added, samples were denatured on a $100^{\circ} \mathrm{C}$ heat block and immediately centrifuged at $15,000 \times \mathrm{g}$ for $10 \mathrm{~min}$. The pellet was removed with a toothpick and the DNA was precipitated by adding $100 \mu \mathrm{l} 7.5 \mathrm{M} \mathrm{NH}_{4} \mathrm{Ac}$ and $400 \mu \mathrm{l}$ isopropanol. The samples were mixed and centrifuged at $15,000 \times \mathrm{g}$ for $30 \mathrm{~min}$ at room temperature. The supernatant was discarded and the dried DNA pellet resuspended in $50 \mu \mathrm{l}$ TE buffer. $5 \mu \mathrm{l}$ of the sample was digested in $50 \mu \mathrm{l}$ volume with 10 units of appropriate restriction enzymes and in the presence of $0.1 \mu \mathrm{g}$ RNase $A$.

\subsubsection{PCR Screens}

PCR screen is a fast way to screen a large number of colonies. For that, a colony was picked and inoculated into $50 \mu \mathrm{LB}$ medium per well of a 96-well plate. The full plate was placed for $3 \mathrm{~h}$ at $37^{\circ} \mathrm{C}$ and then $1 \mu \mathrm{l}$ bacterial culture was added into a new 96-well plate, containing the PCR mix. Primer pairs were designed with one primer lying in the vector backbone and the reverse primer in the insert sequence for the $5^{\prime}$ and the 3 ' cloning site. Clones that give rise to bands of an expected size for both PCR reactions were inoculated into $5 \mathrm{ml}$ LB to grow overnight and process furtherr 


\subsubsection{Cloning strategies for constructs generated and used in this study}

\subsection{BAC DNA retrieval vector PL253}

For generating the BAC DNA retrieval vector PL253, the 5' and 3' GAP repair mini arms were first amplified by PCR A (primer 21137/21138, Annealing $63^{\circ} \mathrm{C}, 445 \mathrm{bp}$ ) and PCR B (primer $21139 / 21140,63^{\circ} \mathrm{C}, 578 \mathrm{bp}$ ), respectively. PCR fragment $\mathrm{A}$ was digested with 20 units of Notl and Hindll and fragment B with Hindll and BamHI in $50 \mu$ final volume for 2 h. $3 \mu \mathrm{g}$ of the PL253 vector was digested with 30 units of Notl and BamHl in $100 \mu \mathrm{l}$ final volume for $3 \mathrm{~h}$ and separated on a $0.7 \%$ TAE agarose gel. 5' ends of the vector DNA were dephosphorylated and the final retrieval vector was generated by ligating $2 \mu \mathrm{l}$ of PL253, 6 $\mu \mathrm{l}$ fragment $A$, and $6 \mu \mathrm{l}$ fragment B. $1 \mu \mathrm{l}$ of the ligation mix was electroporated into E10 cells and $\mathrm{Amp}^{\mathrm{r}}$ colonies selected.

\subsection{IoxP-PGK-EM7-NeobpA-IoxP vector PL452}

For cloning the 5' and 3' GAP repair mini arms amplified by PCR C (primers 19929/19930, Annealing $63^{\circ} \mathrm{C}, 450 \mathrm{bp}$ ) and PCR D (primers 19931/19932, Annealing $63^{\circ} \mathrm{C}, 405 \mathrm{bp}$ ) into PL452, the PCR samples were first cloned into the $\mathrm{PCR} \otimes 2.1-\mathrm{TOPO} \circledast$ vector. Fragment $\mathrm{C}$ was cut out of the vector with 20 units $K p n l$ and $E c o R l$ and fragment $\mathrm{D}$ with BamHl and Notl. $6 \mu \mathrm{g}$ of the vector PL452 was each digested with EcoRI and BamH to obtain a 1.89 $\mathrm{kb}$ large fragment containing the IoxP-PGK-EM7-NeobpA-IoxP cassette and with Kpnl and Notl to obtain a $2.93 \mathrm{~kb}$ vector backbone fragment. Vector fragments were separated on a $0.8 \%$ agarose TAE gel, extracted and dephosphorylated. The final C-loxP-PGK-EM7NeobpA-loxP-D targeting vector PL452 was generated by ligating $2 \mu$ of loxP-PGK-EM7NeobpA-loxP cassette, $2 \mu$ l vector backbone, $5 \mu$ fragment $C$ and $5 \mu$ fragment $D .1 \mu l$ of the ligation mix was electroporated into Electro10 Blue cells and $\mathrm{Amp}^{\mathrm{r}}$ colonies selected. Amp $^{r}$ colonies were screened by PCR analysis (primer pairs 24607/24608 and 24609/24610, Annealing $65^{\circ} \mathrm{C}$ ).

\subsection{FRT-PGK-EM7-NeobpA-FRT-IoxP vector PL451}

For cloning the 5' and 3' GAP repair mini arms amplified by PCR E (primers 19965/19966, Annealing $63^{\circ} \mathrm{C}, 417 \mathrm{bp}$ ) and PCR F (primers 19967/19968, Annealing $63^{\circ} \mathrm{C}, 437 \mathrm{bp}$ ) into PL451, the PCR samples were first cloned into the $P C R \circledast 2.1-T O P O \circledast$ vector. Fragment $E$ 
was cut out of the vector with 20 units $X h o l$ and $E c o R l$ and fragment $F$ with BamHI and Sacll. $6 \mu \mathrm{g}$ of the vector PL451 was digested each with EcoRI and BamHI to obtain a 1.89 $\mathrm{kb}$ large fragment containing the FRT-PGK-EM7-NeobpA-FRT-IoxP cassette and with $X$ hol and Sacll to obtain a $2.94 \mathrm{~kb}$ large vector backbone fragment. Vector fragments were separated on a $0.8 \%$ agarose TAE gel, extracted and dephosphorylated. The final EFRT-PGK-EM7-NeobpA-FRT-IoxP-F targeting vector PL451 was generated by ligating 2 $\mu \mathrm{l}$ of FRT-PGK-EM7-NeobpA-FRT-IoxP cassette, $2 \mu \mathrm{l}$ vector backbone, $5 \mu \mathrm{l}$ fragment $\mathrm{E}$ and $5 \mu \mathrm{l}$ fragment F. $1 \mu \mathrm{l}$ of the ligation mix was electroporated Electro10 Blue cells and $A m p^{r}$ colonies selected. Amp ${ }^{r}$ colonies were screened by PCR analysis (primer pairs $25153 / 25154$ and 25155/24610, Annealing $54^{\circ} \mathrm{C}$ ).

\subsection{Generation of a 3' hybridization probe for Southern Blot Analysis}

The PCR product for the 3' probe (primer 21141/21142, Annealing $60^{\circ} \mathrm{C}, 291 \mathrm{bp}$ ) was cloned into the pCR®2.1-TOPO® vector and cut out of the vector by EcoRl digestion prior Southern Blot hybridization in order to obtain a 291 bp large fragment.

\subsection{Generation of a 5' hybridization probe for Southern Blot Analysis}

The PCR product for the 5' probe (primer $8431 / 8432$, Annealing $50^{\circ} \mathrm{C}, 598 \mathrm{bp}$ ) was cloned into the pBluescript psKII vector and cut out of the vector by Pstl digestion prior Southern Blot hybridization in order to obtain a 536 bp large fragment.

\subsection{Generation of a Neo hybridization probe for Southern Blot Analysis}

The Neo probe was obtained by Pstl and Xbal digestion of the vector PL451, containing the FRT-IoxP-PGK-EM7-NeobpA-FRT-IoxP cassette in a 630 bp large fragment. 
2.3. Cell Cultures

2.3.1. Embryonic Stem Cell Work

2.3.1.1. Materials

\subsection{Chemicals and Reagents}

\begin{tabular}{|c|c|c|}
\hline Product & Company & Cat. No. \\
\hline Amersham Hybond $^{\mathrm{TM}}-\mathrm{N}_{+}$ & GE Healthcare & RPN303B \\
\hline Amersham Rapid-hyb ${ }^{\mathrm{TM}}$ Buffer & GE Healthcare & RPN1635 \\
\hline Bio-Spin 30 Colums & BioRad & $732-6202$ \\
\hline Cell Culture dishes & $\begin{array}{l}\text { Greiner, Costar, Falcon, } \\
\text { Nunc }\end{array}$ & \\
\hline Cymeven (Ganciclovir-Natrium) & Roche Pharma AG & \\
\hline $\mathrm{dCTP},\left[\alpha^{-32} \mathrm{P}\right]-3000 \mathrm{Ci} / \mathrm{mmol} 10 \mathrm{mCi} / \mathrm{ml}$ & Perkin Elmer & NEG513H250UC \\
\hline Dimethyl sulfoxide, Cell Culture Tested & Sigma-Aldrich & D-2650 \\
\hline Dulbecco's PBS (w/o $\mathrm{Ca}^{2+} \& \mathrm{Mg}^{2+}$ ) & PAA & $\mathrm{H} 15-002$ \\
\hline ESGRO-LIF & Chemicon & ESG1107 \\
\hline Fetal Bovine Serum (FBS) & HyClone & SH30070.03 \\
\hline Gelatin from porcine skin & Sigma-Aldrich & G-1890-100 \\
\hline Geneticin (G418-Sulfat) & Gibco & $11811-023$ \\
\hline Hydrochloric Acid (HCL) & Merck & 1.003 .172 .500 \\
\hline Knockout D-MEM & Gibco & 10829-018 \\
\hline Kodak BioMax MS Film & Kodak & 1435726 \\
\hline L-Glutamin $(100 \mathrm{x})$ & Gibco & $25030-024$ \\
\hline MEM non-essential amino acids $(100 \mathrm{x})$ & Gibco & $11140-035$ \\
\hline Mineral oil & Sigma-Aldrich & M-8410-500 \\
\hline Mitomycin C & Sigma-Aldrich & M-0503-2MG \\
\hline N-Lauroylsarcosine sodium salt & Sigma-Aldrich & L-5777 \\
\hline Penicillin/Streptomycin (100x) & Gibco & $15140-122$ \\
\hline Prime-It II Random Primer Labeling Kit & Agilent Technologies & 300385 \\
\hline Proteinase $\mathrm{K}(20 \mathrm{mg} / \mathrm{ml})$ & Qiagen & 19133 \\
\hline Sodium Chloride ( $\mathrm{NaCl})$ & Merck & 1.076 .511 .000 \\
\hline Sodium Hydroxid $(\mathrm{NaOH})$ & Merck & 1.064 .980 .500 \\
\hline Spermidine & Sigma-Aldrich & 82501 \\
\hline Trypsin-EDTA (0.05\%) & Gibco & $25300-054$ \\
\hline Trypsin-EDTA (0.25\%) & Gibco & $25200-056$ \\
\hline$\beta$-Mercaptoethanol "cell culture tested" & Sigma-Aldrich & $\mathrm{M}-7522-100$ \\
\hline
\end{tabular}




\subsection{Equipment}

\begin{tabular}{|l|l|}
\hline Product & Company \\
\hline BIO-RAD Gene Pulser & BIO-RAD \\
\hline Fluo-Link & Biometra \\
\hline
\end{tabular}

\subsection{Media and buffer}

ES cell and MEF medium ( $500 \mathrm{ml}$ Knockout D-MEM, $95 \mathrm{ml} \mathrm{FBS,} 6 \mathrm{ml}$ MEM, $6 \mathrm{ml}$ diluted Bmercaptoethanol, $3 \mathrm{ml}$ Penicillin/Streptomycin, $65 \mu \mathrm{l}$ ESGRO-LIF)

2x Freezing Medium (20\% DMSO, 20\% FBS, 60\% Knockout D-MEM)

100x G418 (360 mg active ingredient in $20 \mathrm{ml} P B S$ )

1000x Ganciclovir (2mM; $510 \mathrm{mg}$ in 1 L PBS)

Lysis Buffer (10 mM Tris, pH 7.5, $10 \mathrm{mM}$ EDTA, pH 8.0, $10 \mathrm{mM} \mathrm{NaCl}, 0.5 \%$ N-Lauroylsarcosine sodium salt, $1 \mathrm{mg} / \mathrm{ml}$ PROTEINASE $\mathrm{K}$

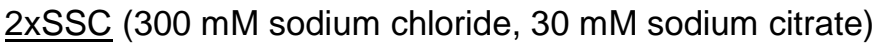

\subsubsection{Methods}

\subsection{Mouse embryonic fibroblast culture}

Mouse embryonic fibroblasts (MEFs) were stored in liquid nitrogen in $500 \mu$ l aliquots. Cells were quickly thawed in a $37^{\circ} \mathrm{C}$ water bath, transferred to a $15 \mathrm{ml}$ tube, and mixed with 10 $\mathrm{ml}$ medium. Cells were centrifuged for $7 \mathrm{~min}$ at $900 \mathrm{rpm}$, resuspended in medium and plated on a gelantine-coated T75 tissue culture flask. P4 - P6 aliquots were split in ratios of $1: 3$, later passages were split (1:2) every $2-3$ days. For passaging cells, the confluent culture was washed once with PBS. Enough trypsin-EDTA $(0.05 \%)$ was added to cover the surface $\left(3.5 \mathrm{ml}\right.$ for a T75 flask). Cells were incubated for $5 \mathrm{~min}$ at $37^{\circ} \mathrm{C}$, an equal volume of medium was added and the cells were triturated. The MEFs were then transferred to the new tissue flasks. To inactivate MEFs, cells were incubated in $10 \mu \mathrm{g} / \mathrm{ml}$ mitomycin $\mathrm{C}$ for $2.5 \mathrm{~h}$ at $37^{\circ} \mathrm{C}$ and then washed three times with PBS. Trypsin-EDTA $(0.05 \%)$ was added and the cells were triturated and finally plated on gelatinized plates. The number of feeder plates was calculated according to area. $1 \mathrm{~T} 175\left(175 \mathrm{~cm}^{2}\right)$ plate was used to make $410 \mathrm{~cm}^{2}$ plates, 5-6 96-well plates or 3-4 6-well plates. MEFs from a T175 plate were frozen in two $500 \mu \mathrm{l}$ aliquots in $1 \times$ Freezing medium. The vials were placed into a freezing container and transferred to a $-80^{\circ} \mathrm{C}$ freezer in order to obtain a slow freezing rate around $1^{\circ} \mathrm{C}$ per minute. After $24 \mathrm{~h}$, cells were transferred into liquid nitrogen for storage. 


\subsection{Embryonic stem cell culture}

Embryonic stem (ES) cells were stored and thawed as described for MEFs and plated onto $6 \mathrm{~cm}^{2}$ feeder plates. The ES cell medium was changed 1-2 times per day and 3-4 $\mathrm{h}$ before passaging. For passaging cells from confluent plates, the medium was aspirated off and the cells were washed once with PBS. Enough trypsin-EDTA (0.25\%) was added to cover surface $\left(500 \mu \mathrm{l}\right.$ for a $6 \mathrm{~cm}^{2}$ plate). Cells were incubated for $15 \mathrm{~min}$ at $37^{\circ} \mathrm{C}$, an equal volume of ES cell medium was added and the cells were carefully triturated with a pasteur pipette. Cells were usually split (1:6) onto fresh feeder plates. ES cells were frozen in $1 \times$ Freezing medium at a density of $3.0 \times 10^{7}$ cells $/ \mathrm{ml}$, making a total of $9 \times 10^{6}$ cells per $300 \mu \mathrm{l}$ aliquot. The vials were placed into a freezing container and transferred to a $-80^{\circ} \mathrm{C}$ freezer in order to obtain a slow freezing rate around $1^{\circ} \mathrm{C}$ per minute. After $24 \mathrm{~h}$, cells were transferred into liquid nitrogen for storage.

\subsection{ES cell electroporation}

The ES cells were passaged (1:2) $24 \mathrm{~h}$ before electroporation and the medium was changed $3 \mathrm{~h}$ prior harvesting. The cells were trypsinized as described earlier and resuspended in media. The cells were centrifuged for for $7 \mathrm{~min}$ at $900 \mathrm{rpm}$ and resuspended in $10 \mathrm{ml}$ PBS. The total number of cells was determined by counting $20 \mu \mathrm{l}$ of a 1:10 dilution. Cells were pelleted again and resuspended in PBS at a density of $11 \mathrm{x}$ $10^{6} \mathrm{cells} / \mathrm{ml} .900 \mu \mathrm{l}$ aliquots of cells were prepared and $(25-50 \mu \mathrm{g})$ linearized vector DNA was added. Electroporation into ES cells was performed using a BIO-RAD Gene Pulser at $2.3 \mathrm{kV}$ and $500 \mu \mathrm{F}$. The cuvette was left at room temperature for $5 \mathrm{~min}$ and cells were then plated onto $10 \mathrm{~cm}^{2}$ feeder plates. Cells were selected against G418 (positive selection) from $24 \mathrm{~h}$ post-electroporation onwards and selected against Ganciclovir between 3-5 days post-electroporation (negative selection).

\subsection{Picking ES cell colonies}

ES cell colonies for later analysis by Southern Blot were picked 11 - 12 days after electroporation. $25 \mu$ trypsin-EDTA $(0.25 \%)$ was added into each well of a 96 -well roundbottom plate and kept on ice. ES cell medium was aspirated from the plate and substituted by PBS. Colonies were picked by sucking the colony into a $10 \mu$ pipette tip and was transferred into the trypsin ( 1 colony per well). After completing a 96-well plate, the cells were incubated for $15 \mathrm{~min}$ at $37^{\circ} \mathrm{C} .35 \mu \mathrm{l} \mathrm{ES}$ cell medium was added per well, the 
cells were triturated and transferred to a flat-bottom feeder plate and cultured in $150 \mu \mathrm{l}$ medium per well. Cells were split 1:1 after a few days to obtain a higher cell density. Finally, cells were split (1:3) onto two feeder plates for freezing and onto one gelantinecoated plate for subsequent Southern Blot analysis.

\subsection{Freezing 96-well plates}

To freeze 96-well plates, cells were trypsinized in $50 \mu \mathrm{l}$ trypsin-EDTA (0.25\%) for $15 \mathrm{~min}$ at $37^{\circ} \mathrm{C} .50 \mu \mathrm{l} 2 \times$ Freezing Medium was added to each well and the cells were triturated. $100 \mu \mathrm{l}$ sterile filtered mineral oil was added to each well, the plate was sealed with parafilm and placed into a small polystyrene box and transferred to a $-80^{\circ} \mathrm{C}$ freezer.

\subsection{Isolating ES cell DNA on 96-well Plates}

ES cell clones cultured on gelantine-coated 96-well plates were washed twice with PBS and then lysed in $50 \mu \mathrm{l}$ Lysis Buffer. Plates were kept for $6-8 \mathrm{~h}$ at $60^{\circ} \mathrm{C}$ in a humified chamber. Then, $100 \mu \mathrm{l}$ of $75 \mathrm{mM} \mathrm{NaCl}$ in cold $100 \%$ ethanol was added to each well and the DNA was precipitated for $30 \mathrm{~min}$ at room temperature. The plates were briefly centrifuged, then washed three times with $70 \%$ ethanol and air-dried for $30 \mathrm{~min}$. ES cell DNA was digested overnight at $37^{\circ} \mathrm{C}$ in $30 \mu$ volume containing $1 \mathrm{mM}$ spermidine, 100 $\mu \mathrm{g} / \mathrm{ml}$ BSA and $20 \mu \mathrm{l}$ units of enzyme in $1 \mathrm{x}$ restriction buffer. $4 \mu \mathrm{l}$ of loading buffer was added the next day to each well and samples were loaded onto a $0.8 \%$ TAE agarose gel and run for $5 \mathrm{~h}$ at $60 \mathrm{~V}$.

\subsection{Southern Blot (Southern, 1975)}

Southern blot analysis is a method for the detection of specific genomic DNA sequences. First, DNA samples are transferred of electrophoresis-separated DNA fragments to a filter membrane and the subsequent labeling of a specific fragment by e.g. a hybridization probe.

In order to transfer the DNA samples from the $0.8 \%$ TAE agarose gel, the gel was washed in $0.25 \mathrm{M} \mathrm{HCl}$ for $10 \mathrm{~min}$ to depurinate the DNA for a better transfer. The gel was then washed in $0.4 \mathrm{M} \mathrm{NaOH}$ to denaturate the DNA and to produce sDNA single strands. The 
DNA was then capillary transferred overnight from the gel in $0.4 \mathrm{M} \mathrm{NaOH}$ blotting solution onto a positively charged nylon membrane. A glass plate was placed above two plastic containers filled with blotting solution. A long sheet of Whatman paper was soaked in blotting solution and placed on the plate, each end reaching the blotting solution in the plastic containers. Then the gel was then placed on the Whatman paper and the nylon membrane placed on top of the gel, followed by three sheets of wet Whatman paper. A large pile of tissue was added and a weight was positioned on top of the construction. The next day, the membrane was neutralized in 2xSSC, air-dried and the DNA was covalently cross-linked by exposure to ultraviolet irradiation (1.0 J/cm2; Fluo-Link).

The hybridization of the probes was performed with the Prime It Random Primer Labeling Kit. For detailed protocol refer to the product manual. Briefly, 25 ng probe DNA was mixed with random oligonucleotide primers, $\left[{ }^{32} \mathrm{P}\right] \mathrm{dCTP}$ labeling buffer, $50 \mu \mathrm{Ci}$ labeled nucleotide $\left(\left[{ }^{32} \mathrm{P}\right] \mathrm{dCTP}\right)$ and 5 units of Exo(-) Klenow polymerase in $50 \mu$ l volume. Reaction mix was incubated at $37^{\circ} \mathrm{C}$ for $30 \mathrm{~min}$ and purified over a Bio-Spin $30 \mathrm{SSC}$ columns. The probe was then denatured for $5 \mathrm{~min}$ on a $100^{\circ} \mathrm{C}$ heat block and placed immediately onto ice. The membrane was pre-incubated in a glass bottle at $65^{\circ} \mathrm{C}$ with Rapid-hyb buffer for at least $15 \mathrm{~min}$ and then the probe was added at a final concentration of $2 \mathrm{ng} / \mathrm{ml}$. The membrane was hybridized for $2 \mathrm{~h}$ at $65^{\circ} \mathrm{C}$ and then washed once with $2 \times S S C, 0.1 \%$ SDS for $20 \mathrm{~min}$ at room temperature and twice with $1 \times$ SSC, $0.1 \%$ SDS and twice with $0.5 x S S C, 0.1 \%$ SDS at $65^{\circ} \mathrm{C}$. The washed membrane was wrapped in cling film and autoradiographed at $80^{\circ} \mathrm{C}$ overnight. 


\subsubsection{Mouse hippocampal Neuron Culture}

\subsubsection{Materials}

\subsection{Chemicals and Reagents}

\begin{tabular}{|l|l|l|}
\hline Product & Company & Cat. No. \\
\hline Albumin from bovine serum (BSA) & Sigma & A4503 \\
\hline B-27® Serum-Free Supplement (50X) & Gibco & $17504-044$ \\
\hline Calcium chloride dihydrate & Sigma & C3881 \\
\hline Dulbecco's Modified Eagle Medium (DMEM) & Gibco & $41966-029$ \\
\hline EDTA & Sigma & E1644 \\
\hline Fetal Bovine Serum (FBS) & PAA & A15-104 \\
\hline Glutamax (200mM) & Gibco & 35050038 \\
\hline $\begin{array}{l}\text { Hank's Balanced Salt Solution , no Ca }{ }^{2+}, \text { no } \text { Mg }^{2+} \\
\text { (HBSS) }\end{array}$ & Gibco & $14170-088$ \\
\hline L-Cysteine & Sigma & C7352 \\
\hline Millex Syringe Filter Units, 0.22 $\mu \mathrm{m}$ & Gibco & SLGP033RS \\
\hline Neurobasal®-A Medium (1X), Liquid & Gibco & $10888-022$ \\
\hline Papain & Worthington Biochemical & LS003126 \\
\hline Penicillin/Streptomycin (100x) & Gibco & $15140-122$ \\
\hline Poly-L-lysine & Sigma & P4707 \\
\hline Trypsin inhibitor from chicken egg white & Sigma & T9253 \\
\hline
\end{tabular}

\subsection{Media and Solutions}

Papain Solution $(0.2 \mathrm{mg} / \mathrm{ml}$ cysteine, $1 \mathrm{mM} \mathrm{CaCl}, 0.5 \mathrm{mM}$ EDTA in DMEM and 25 units Papain per ml; the Papain Solution was bubbled with carbogen (95\% oxygen, 5\% carbon dioxide) for 20 min and sterile filtered $(0.2 \mu \mathrm{m})$ prior use)

Stop Solution ( $2.5 \mathrm{mg} / \mathrm{ml} \mathrm{BSA}, 2.5 \mathrm{mg} / \mathrm{ml}$ trypsin inhibitor, 10\% FBS in D'MEM)

Complete Neurobasal Medium (500 ml Neurobasal-A, 5 ml GlutaMAX I, 10 ml B27-supplement, 1 $\mathrm{ml}$ Penicillin/Streptomycin)

\subsubsection{Methods}

\subsection{Treatment of coverslips for mouse hippocampal neuron cultures}

Glas coverslips (32 mm) were washed first in $1 \mathrm{M} \mathrm{HCl}$ overnight, then multiple times with $\mathrm{dd}_{2} \mathrm{O}$ and finally with $70 \%$ Ethanol. Coverslips were then transferred into the wells of a 6well cell culture plate and UV-sterilized for 1 hour. Glass coverslips were coated in a 1:12 
dilution of Poly-L-Lysine in PBS overnight at $37^{\circ} \mathrm{C}$. The coverslips were then washed twice with PBS and incubated with the Complete Neurobasal Medium until further use.

\subsection{Primary mouse hippocampal neuron culture}

Neonatal P0 mouse pups were decapitated and the brains were placed into HBSS. The hippocampi were removed, transferred into $500 \mu \mathrm{l}$ of pre-warmed Papain solution and incubated at $37^{\circ} \mathrm{C}$ with gentle agitation for one hour. Then, the supernatant was removed and $500 \mu \mathrm{l}$ of pre-warmed Stop Solution was added to the hippocampal tissue and kept at $37^{\circ} \mathrm{C}$ for an additional 15 minutes. Then, the supernatant was removed and the tissue was washed once with $500 \mu \mathrm{l}$ Complete Neurobasal Medium. Then the tissue was triturated gently for 15 times in $200 \mu \mathrm{l}$ of Complete Neurobasal Medium and the tube was kept at room temperature for 2 minutes and then the supernatant was transferred into a fresh tube containing $800 \mu \mathrm{l}$ of pre-warmed Complete Neurobasal Medium. A second trituration step was perforemd to increase the number of cells for high-density cultures, if necessary. Cells were counted on a Naubauer Counting Chamber and approximately 100,000 to 200,000 cells were plated per well of a six-well plate for continental cultures. The neurons were infected on DIV1 by adding the virus directly into the culture medium. The medium was changed 15-24 hours after infection to prevent neurotoxicity of the virus. The expression level of green fluorescent protein (GFP) was used in order to determine the viral titer.

For autaptic neuron cultures, approximately 4,000 neurons were plated per well of a sixwell plate that contained small astrocyte feeder islands on glass coverslips, a method that results in the isolation of individual neurons (Bekkers, 1991). The preparation of the astrocyte microislands was performed by Ines Beulshausen and Sabine Bolte as published (Burgalossi et al., 2012). Electrophysiological experiments on autpaptic cultures in order to assess physiological consequences of Cre-mediated deletion of Munc13-1 in neurons were performed by Dr. Riikka Liisa Uronen. 


\subsubsection{HEK 293FT cell culture}

\subsubsection{Materials}

\subsection{Chemicals and Reagents}

\begin{tabular}{|l|l|l|}
\hline Product & Company & Cat. No. \\
\hline Amicon Ultra-15 Centrifugal Filter Unit & Millipore & UFC901024 \\
\hline Butyric acid & Sigma & 303410 \\
\hline DMEM, High Glucose, GlutaMAXTM, Pyruvate & Gibco & $31966-021$ \\
\hline Dulbecco's PBS (w/o Ca ${ }^{2+}$ Mg $^{2+}$ ) & PAA & H15-002 \\
\hline Fetal Bovine Serum (FBS) & PAA & A15-104 \\
\hline Geneticin, G 418 Sulfate, Cell Culture Tested & Calbiochem & 345810 \\
\hline HEK293FT cell line & Invitrogen & R700-07 \\
\hline HEPES, 1M Buffer Solution & Gibco & $15630-080$ \\
\hline Lipofectamine® 2000 Transfection Reagent & Invitrogen & $11668-500$ \\
\hline Millex-HA Syringe Filter Units, 0.45 $\mu m$ & Millipore & SLHA033SB \\
\hline Neurobasal®-A Medium (1X), Liquid & Gibco & $10888-022$ \\
\hline Opti-MEM® I Reduced Serum Medium & Gibco & $31985-047$ \\
\hline Penicillin/Streptomycin (100x) & Gibco & $15140-122$ \\
\hline Poly-L-lysine & Sigma & P4707 \\
\hline Trypsin-EDTA (0.05\%) & Gibco & $25300-054$ \\
\hline
\end{tabular}

\subsection{Media and buffer}

HEK293FT Medium (500 ml DMEM containing GlutaMAX, 50 ml FBS, 5 ml Pen/Strep)

\subsubsection{Methods}

\subsection{HEK293FT culture}

The human embryonic (HEK) 293FT cell line was used for the production of lentiviral particals. HEK293FT cells express the SV40 large $T$ antigen under the human cytomegalovirus (CMV) promotor to facilitate lentiviral production together with the neomycin resistance gene from the pCMVSPORT6Tag.neo plasmid (Invitrogen). HEK293FT cell cultures were therefore kept in cell culture dishes in HEK293FT Medium containing $500 \mu \mathrm{g} / \mathrm{ml}$ Geneticin for selection at $37^{\circ} \mathrm{C}$ and $5 \% \mathrm{CO}$. 


\subsection{Thawing of HEK293FT cells}

A vial of frozen HEK293FT cells was quickly thawed and transferred into a $15 \mathrm{ml}$ Falcon tube containing HEK293FT Medium. Cells were spun down for $5 \mathrm{~min}$ at $1.500 \mathrm{rpm}$ and resuspended in HEK293FT medium and plated onto cell culture dishes. After 24 hours, the medium was replaced by fresh medium containing $500 \mu \mathrm{g} / \mathrm{ml}$ Geneticin.

\subsection{Passaging of HEK293FT cells}

For passaging cells, the confluent culture plate was washed once with PBS. Enough trypsin-EDTA (0.05\%) was added to cover the surface (2 $\mathrm{ml}$ on a $10 \mathrm{~cm} 2 \mathrm{dish}$ ). Cells were incubated briefly at room temperature, then an equal volume of medium was added and the cells were triturated and plated onto cell culture dishes and cultured in the presence of $500 \mu \mathrm{g} / \mathrm{ml}$ Geneticin.

\subsection{Production of lenti-viral particles in HEK293FT cells}

In order to produce viral particles, HEK293FT cells were split in a 1:2 ratio 24 hours prior transfection onto PLL-coated $15 \mathrm{~cm}^{2}$ cell culture plates. Semiconfluent HEK293FT cell cultures were co-transfected with the vector plasmids ( $p F U G W$ or pFUGW-iCre) together with a plasmid (PACK) encoding for proteins that form the viral core structure (GAG group antigens) and for the reverse transcriptase and integrase (POL), as well as with a plasmid (ENV) encoding for the vesicular stomatitis virus glycoprotein (VSV-G) forming the viral envelope. For a $15 \mathrm{~cm}^{2}$ cell culture dish, $60 \mu \mathrm{l}$ Lipofectamine2000 was added to 2 $\mathrm{ml}$ of OPTI-MEM and kept at room temperature for $5 \mathrm{~min}$. The transfection mix was prepared by adding $40 \mu \mathrm{g}$ vector plasmid, $16 \mu \mathrm{g}$ PACK plasmid and $16 \mu \mathrm{g}$ ENV plasmid to $2 \mathrm{ml}$ OPTI-MEM. Then, the Lipofectamine2000 in OPTI-MEM was added to the transfection mix and kept at room temperature in the dark for $60 \mathrm{~min}$. The HEK293FT cell Medium was replaced by $11 \mathrm{ml}$ OPTI-MEM with 10\% FCS and the Transfection Mix was added to the cells. 20 hours after transfection, the medium was replaced by DMEM containing $2 \%$ FBS and $10 \mathrm{mM}$ sodium butylate. 48 hours after transfection, the viral particles were harvested. The supernatant of the cell culture plates was filtered through $0.45 \mu \mathrm{m}$ pores and concentrated using an Amicon filter system by centrifugation with $4,300 \mathrm{rpm}$ at $4^{\circ} \mathrm{C}$ for $10 \mathrm{~min}$. The concentrated virus was then washed twice with cool 
Neurobasal-A medium. Finally, small aliquots of virus particles inNeurobasal-A medium and in the presence of $100 \mathrm{mM}$ HEPES were frozen in liquid nitrogen and stored at $-80^{\circ} \mathrm{C}$.

\subsubsection{Organotypic Slice Culture}

\subsubsection{Materials}

\subsection{Chemicals and Reagents}

\begin{tabular}{|l|l|l|}
\hline Product & Company & Cat. No. \\
\hline 5-Fluoro-2'-deoxyuridine & Sigma & F0503 \\
\hline BME (Basal Medium Eagle without Glutamin) & Gibco & 41010026 \\
\hline Cytosine $\beta$-D-arabinofuranoside hydrochloride (Ara-C) & Sigma & C6645 \\
\hline Glucose x H2O & Fluka & 49159 \\
\hline Glutamax (200mM) & Gibco & 35050038 \\
\hline $\begin{array}{l}\text { Hank's Balanced Salt Solution , no } \mathrm{Ca}^{2+}, \text { no } \mathrm{Mg}^{2+} \\
\text { (HBSS) }\end{array}$ & Gibco & $14170-088$ \\
\hline Heat-inactivated horse serum (HS) & Gibco & $26050-088$ \\
\hline Kynurenic Acid & Tocris & 223 \\
\hline Millex Syringe Filter Units, 0.22 $\mu \mathrm{m}$ & Gibco & SLGP033RS \\
\hline Millicell Cell Culture Inserts & Millipore & PICM03050 \\
\hline Millipore confetti & Millipore & FHLC04700 \\
\hline Minimum Essential Medium (MEM) & Gibco & $11700-077$ \\
\hline razor blades & Gillette & \\
\hline Uridine & Sigma & U3750 \\
\hline
\end{tabular}

\subsection{Equipment}

\begin{tabular}{|l|l|}
\hline Product & Company \\
\hline Mclllwain tissue chopper & The Mickle Engineering Company Ltd, UK \\
\hline
\end{tabular}

\subsection{Media and buffer}

2xMEM $\left(9.39 \mathrm{~g} / 500 \mathrm{ml} \mathrm{ddH}_{2} \mathrm{O}\right)$

Culture Medium (22.44ml ddH $\mathrm{m}_{2}$, $25 \mathrm{ml}$ 2xMEM, $25 \mathrm{ml} \mathrm{BME,} 1 \mathrm{ml}$ Glutamax, 1.56ml Glucose 40\%, $25 \mathrm{ml} \mathrm{HS}$ )

Cutting solution (pH 7.4; 97 ml HBSS, 2.5 ml Glucose 20\%, 1 ml 100mM Kynurenic Acid)

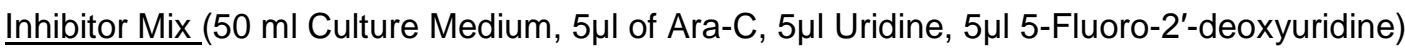




\subsubsection{Methods}

\subsection{Preparation of organotypic hippocampal slice cultures}

The protocol for the preparation of organotypic hippocampal slice cultures was based on the "interface method" introduced by Stoppini and colleagues in 1991 (Kerr et al., 2008; Stoppini et al., 1991). Control and knockout mice were taken from the same mouse colony and from the same litters. For E18 preparations, the mothers were anesthetized, decapitated and the pups taken out by cesarean section. E18-P0 pups were then decapitated and the brains were placed into Cutting Solution. The hippocampi, still attached to the entorhinal cortex, were removed and transferred with a sterile Pasteur pipette onto the Tissue Culture Stage under a preparation hood. Excess liquid was removed with sterile autoclaved tissues. Slices of $300 \mu \mathrm{m}$ thickness were cut with a Mclllwain tissue chopper perpendicular to the longitudinal axis of the hippocampus. Then the stage was flooded with Cutting Solution and the slices were transferred in liquid with a plastic Pasteur pipette into a plastic dish. Only undamaged slices were then placed onto small membrane pieces (confetti) on top of the microporous cell culture membrane. Usually, maximally four hippocampal slices were cultured on each cell culture insert. Slices were maintained with culture medium. The medium was first changed 24h after preparation and then 2-3 times per week. Between 3-5 days in-vitro (DIV) $100 \mu \mathrm{M}$ cytosine arabinoside, $100 \mu \mathrm{M}$ uridine and $100 \mu \mathrm{M}$ 5-fluorodeoxyuridine were added for $24 \mathrm{~h}$ to the culture. The slices were maintained at $37^{\circ} \mathrm{C}$ and in $5 \% \mathrm{CO}_{2}$ for $2-4$ weeks. High-pressure-freezing fixation for electron tomography was performed between 4 and 5 weeks in vitro. The animals were genotyped by using tail DNA and subsequent PCR analysis. In addition, for most of the experiments, the rest of the brain was frozen in liquid nitrogen and kept for Western-Blot analysis of the genotype if necessary. 


\subsection{Biochemistry}

\subsubsection{Materials}

\subsubsection{Chemicals and reagents}

\begin{tabular}{|c|c|c|}
\hline Product & Company & Cat. No. \\
\hline $\begin{array}{l}\text { Acrylamide/N,N'-Methylene-bis-Acrylamide (37.5:1) } \\
\text { (AMBA) }\end{array}$ & National Diagnostics & EC-890 \\
\hline Amersham HyperfilmTM ECL & GE Healthcare & 28906837 \\
\hline Ammonium persulfate (APS) & Sigma-Aldrich & A-6761 \\
\hline Aprotinin & Roth & A162.3 \\
\hline Bio-Rad Protein Assay Dye Reagent & BIO-RAD & $500-0006$ \\
\hline Bromophenol Blue & Pierce & 20730 \\
\hline Cell scraper & Nunc & 179693 \\
\hline Dithiothreitol (DTT) & Boehringer Ingelheim & 708992 \\
\hline ECL Reagent (Kit) & Amersham, GE-Healthcare & $\begin{array}{l}\text { RPN2106V1 } \\
\text { and }-2\end{array}$ \\
\hline Ethylenediaminetetraacetic Acid Trisodium Salt (EDTA) & Merck & 1.084 .180 .250 \\
\hline Glacial acetic acid & Merck & 1.000 .632 .511 \\
\hline Glycine & Sigma-Aldrich & 33226 \\
\hline Goat Serum & Gibcro, Invitrogen & $16210-072$ \\
\hline IGEPAL & Sigma-Aldrich & 13021 \\
\hline Instant Milk Powder & Granovita $\mathrm{GmbH}$ & \\
\hline Leupeptin & Pepta Nova & 4041 \\
\hline MemCodeTM Reversible Protein Stain Kit & Thermo Scientific & 24580 \\
\hline Methanol & J.T. Baker & 8402 \\
\hline N,N,N',N'-Tetramethyl-ethylenediamine (TEMED) & Serva & 35925 \\
\hline $\begin{array}{l}\text { N-2-Hydroxyethylpiperazine- N'-2-ethane sulfonic acid } \\
\text { (HEPES) }\end{array}$ & Biomol & 5288 \\
\hline PageRulerTM Prestained Protein ladder & Fermentas & SM0671 \\
\hline Phenylmethylsulfonylfluorid & Sigma-Aldrich & P7626 \\
\hline Ponceau-S & Sigma-Aldrich & 81460 \\
\hline $\begin{array}{l}\text { Protran BA83 Nitrocellulose Blotting Membrane, } 0.2 \mu \mathrm{m} \text {, } \\
82 \mathrm{~mm}\end{array}$ & GE Healthcare & 10401396 \\
\hline Sodium dodecylsulfate (SDS) & GEBRU & 1012 \\
\hline Sucrose & Merck & 1.076 .511 .000 \\
\hline Tris-Base & Sigma-Aldrich & T1503 \\
\hline Tween 20 & Sigma-Aldrich & P7949 \\
\hline
\end{tabular}




\subsubsection{Equipment}

\begin{tabular}{|l|l|}
\hline Product & Company \\
\hline Bio-Rad mini gel electrophoresis system (protean II) & Bio Rad \\
\hline BioScience Ultrospec3100pro & Amersham \\
\hline Blotting system, TE22 Mighty Snall tank transfer & Hoefer \\
\hline POTTER'S Homogenizer & B.Braun \\
\hline
\end{tabular}

\subsubsection{Media and buffer}

Homogenization Buffer: (320mM sucrose, 5mM HEPES (pH7.4), 1mM EDTA, 0.1 $\mu$ M Aprotinin,

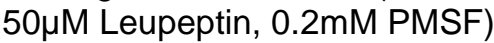

Lysis Buffer: (320mM Sucrose, $10 \mathrm{mM}$ Tris- $\mathrm{HCl}(\mathrm{pH} 7.5), 5 \mathrm{mM}$ HEPES (pH7.4), $150 \mathrm{mM} \mathrm{NaCl}, 1 \mathrm{mM}$

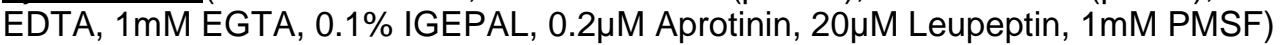

3x SDS-sample buffer: (10\% SDS, $140 \mathrm{mM}$ Tris-HCL (pH 6.8), 3mM EDTA, 30\% Sucrose, $100 \mathrm{mM}$ DTT, $0.1 \%$ bromophenolblue, $150 \mathrm{mM}$ DTT)

Upper stacking gel (5 \% AMBA, 125 mM Tris-HCL (pH 6.8), 0.1\% SDS, 0.05\% APS, $0.005 \%$ TEMED)

Lower resolving gel (7-15\% AMBA, $325 \mathrm{mM}$ Tris-HCL (pH 8.8), 0.1\% SDS, 0.05\% APS, $0.005 \%$ TEMED)

Running buffer(25mM Tris- $\mathrm{HCl}, 250 \mathrm{mM}$ Glycine, $0.1 \%$ SDS, pH 8.8)

Transfer Buffer:(25mM Tris-Base, $190 \mathrm{mM}$ Glycine, $20 \%$ Methanol)

TBS:(10mM Tris- $\mathrm{HCl}, 150 \mathrm{mM} \mathrm{NaCl}, \mathrm{pH} 7.5)$

Blocking buffer:(5\% milk powder, 5\% Goat Serum, 9.1/ Tween 20 in TBS)

Washing Buffer $(0.1 \%$ Tween 20 in TBS)

Ponceau Red:(0.1\% Ponceau, 5\% glacial acetic acid in ddH20)

\subsubsection{Antibodies}

\begin{tabular}{|l|l|l|}
\hline Product / concentration & Company / Reference & Cat. No. \\
\hline $\begin{array}{l}\text { anti-Munc13-1 (affinity purified rabbit polyclonal, N- } \\
\text { terminal \#40); 1:1000 }\end{array}$ & $\begin{array}{l}\text { (Varoqueaux et al. 2005; } \\
\text { Cooper et al. 2012) }\end{array}$ & \\
\hline $\begin{array}{l}\text { anti-Munc13-1 (affinity purified rabbit polyclonal, C- } \\
\text { terminal \#N395); 1:250 }\end{array}$ & (Betz et al., 1997) & TUB 2.1 \\
\hline $\begin{array}{l}\text { anti-ß-Tubulin (mouse monoclonal) 1:5000 } \\
\text { 1:10000 }\end{array}$ & Sigma & 101011 \\
\hline
\end{tabular}

Secondary goat anti-mouse and anti-rabbit affinity purified antibodies conjugated to horse radish peroxidase (HRP) (Bio Rad) were used in this study at a concentration of 1:10000. 


\subsubsection{Methods}

\subsubsection{Mouse Brain Homogenate}

P0 mice were killed by decapitation and the brains were removed and each transferred into $1 \mathrm{ml}$ ice-cold Homogenization buffer. The brain tissue was homogenized at $900 \mathrm{rpm}$ with a glas/Teflon homogenizer at $4^{\circ} \mathrm{C}$ and collected for Western Blot analysis.

\subsubsection{Cell Culture Homogenization}

To check for changes in protein levels of lentiviral infected continental mouse hippocampal neuron cultures, neurons were washed twice with PBS and then incubated for 10min on ice in Lysis buffer. Samples were transferred to Eppendorf cups, centrifuged at 900x gmax for 10 minutes and the supernatant was collected and used for Western Blot analysis.

\subsubsection{Preparation of Proteins Samples for SDS Electrophoresis}

The protein concentration was determined using the Bradford Assay, measuring the absorbance of the sample at $595 \mathrm{~nm}$ wavelength with a BioScience Ultrospec3100pro spectrophotometer (Bradford, 1976). For a detailed protocol refer to the "Bio-Rad Protein Assay" manual. For all the biochemical experiments, samples were standardized to the same concentration in Homogenization- or Lysis buffer. The protein samples were then dissolved in SDS sample buffer to a $1 \mathrm{x}$ final concentration and boiled at $95^{\circ} \mathrm{C}$ for 5 minutes.

\subsubsection{SDS-PAGE (Laemmli 1970) and Western Blotting (Towbin 1979; Burnette 1981)}

In order to separate proteins based on their molecular seize, sodium dodecyl sulfate polyacrylamide gel electrophoresis (SDS-PAGE) was performed under denaturing conditions. SDS as an anionic detergent linearizes proteins and adds a negative charge to them (Schägger, 2006). Negatively charged proteins will migrate in an electric field 
through a porous gel towards the anode. Briefly, samples in SDS sample buffer were loaded onto a two-layer Tris-glycine gel (stacking- and resolving gel) in a Bio-Rad mini gel electrophoresis system filled with running buffer and ran with $20 \mathrm{~mA}$ in the stacking-and with $30 \mathrm{~mA}$ in the resolving gel until the dye ran out of the gel.

The Western Blot technique was used to transfer the separated protein samples after SDS-PAGE onto a nitrocellulose membrane. Proteins were elecrophoretically transferred from the acrylamide gel onto a nitrocellulose membrane for 600 milliampere-hours (mAh) in transfer buffer in a tank blotting system.

The blotting efficiency was monitored by subsequent reversible Ponceau-staining or by using the MemCodeTM Reversible Protein Stain Kit if low protein concentrations had been loaded. The nitrocellulose membrane was then washed once with $\underline{\text { TBS }}$ and then incubated in the blocking buffer for $30 \mathrm{~min}$ at room temperature with gentle agitation. The blot was then incubated with a primary antibody that was designed to specifically detect an epitope in the protein of interest for either 1 hour at room temperature or overnight at $4^{\circ} \mathrm{C}$. The membrane was then washed multiple times with the washing buffer before the blot was incubated with a secondary antibody conjugated to HRP. The membrane was washed again multiple times and immune-reactive protein bands were then visualized with an enhanced chemi-luminescence (ECL) detection system. In this reaction, the HRP catalyzes the oxidation of luminol in the presence of the oxidizing agent hydrogen peroxide, which results in the emission of light with a $428 \mathrm{~nm}$ wavelength. The signal can then be detected and visualized on Amersham Hyperfilm films.

\subsection{Immunostaining procedures}

\subsubsection{Materials}

\subsubsection{Chemicals and reagents}

\begin{tabular}{|l|l|l|}
\hline Product & Company & Cat. No. \\
\hline Albumin from bovine serum $(\mathrm{BSA})$ & Sigma & A4503 \\
\hline Aqua Poly Mount & Polysciences & 18606 \\
\hline Disodium phosphate $\left(\mathrm{Na}_{2} \mathrm{HPO}_{4}\right)$ & Sigma & S9763 \\
\hline Fish Skin Gelatin & Sigma & $\mathrm{G} 7041$ \\
\hline Goat Serum & Gibcro, Invitrogen & $16210-072$ \\
\hline Monopotassium phosphate $\left(\mathrm{KH}_{2} \mathrm{PO}_{4}\right)$ & Merck & 1.048 .731 .000 \\
\hline
\end{tabular}




\begin{tabular}{|l|l|l|}
\hline Product & Company & Cat. No. \\
\hline Paraformaldehyde (PFA) & Serva & 3.1628 .02 \\
\hline Potassium chloride (KCl) & Merck & 1.049 .361 .000 \\
\hline Sodium Chloride $(\mathrm{NaCl})$ & Merck & 1.076 .511 .000 \\
\hline Triton-X-100 & Roche & 10789704001 \\
\hline
\end{tabular}

\subsubsection{Equipment}

\begin{tabular}{|l|l|}
\hline Product & Company \\
\hline LAS AF Software & Leica \\
\hline TCS SP5 Confocal Microscope & Leica \\
\hline
\end{tabular}

\subsubsection{Media and Solutions}

Incubation Solution 1: 10\% goat serum, $0.3 \%$ Triton-X 100, 0.1\% fish skin gelatin in PBS Incubation Solution 2: $1 \%$ goat serum, $0.1 \%$ Triton-X-100 in PBS

Blocking Solution: $20 \%$ BSA; $0.1 \%$ Triton-X-100 in PBS

Permeabilization Solution: $1 \%$ Triton-X-100 in PBS

1x PBS: $137 \mathrm{mM} \mathrm{NaCl}, 2.7 \mathrm{mM} \mathrm{KCl} ; 10 \mathrm{mM} \mathrm{Na}_{2} \mathrm{HPO}_{4} ; 1.8 \mathrm{mM} \mathrm{KH}_{2} \mathrm{PO}_{4}$

\subsubsection{Antibodies}

\begin{tabular}{|l|l|l|}
\hline Product / concentration & Company / Reference & Cat. No. \\
\hline SNAP23 (rabbit polyclonal antiserum) 1:2000 & Synaptic Systems & 111202 \\
\hline SNAP25 (purified mouse monoclonal, 71.1) 1:600 & Synaptic Systems & 111011 \\
\hline $\begin{array}{l}\text { VGLUT1 (guinea pig polyclonal antiserum) } \\
\text { Sy000 - 1:2000 }\end{array}$ & Synaptic Systems & 135304 \\
\hline $\begin{array}{l}\text { Synaptobrevin-1 (rabbit polyclonal antiserum) 1:400 } \\
\text { 1:200 }\end{array}$ & Synaptic Systems & 104002 \\
\hline MAP2 (chicken polyclonal antiserum) 1:1000 & Synaptic Systems & 104211 \\
\hline Munc13-1 (affinity purified rabbit polyclonal) 1:1000 & Novus Biologicals & NB300-213 \\
\hline
\end{tabular}

Secondary antibodies were coupled to Alexa 488, 555, 633 fluophores (Invitrogen) and were used in a concentration of 1:1300. 


\subsubsection{Methods}

\subsubsection{Immunocytochemistry on autaptic hippocampal neurons}

Hippocampal autaptic neuron cultures were fixed for 20 min in 4\% PFA in PBS. After three 5 minutes washing steps, coverslips were incubated for one hour in incubation solution without antibodies to block non-specific binding sites. Then, coverslips were incubated for two hours at room temperature with the primary antibodies diluted in incubation solution 1, washed three times with PBS and afterwards incubated in secondary antibodies diluted in incubation solution for one hour at room temperature. After additional three washes in PBS, coverslips were mounted on object slides and stored at $4^{\circ} \mathrm{C}$ prior imaging.

\subsubsection{Immunohistochemistry on hippocampal organotypic slices}

Immunohistochemistry, imaging and image processing of hippocampal organotypic organotypic slices was performed by Dr. Benjamin Cooper. Hippocampal organotypic slices were fixed in $4 \%$ paraformaldehyde in PBS overnight at $4^{\circ} \mathrm{C}$. Slices were then washed three times in PBS and then carefully separated from the small membrane confetti with a thin brush. Subsequently, slices were incubated in permeabilization solution to allow better antibody penetration overnight at $4^{\circ} \mathrm{C}$. Slices were then transferred into blocking solution and incubated for one hour at room temperature. Subsequently, slices were incubated in diluted primary antibodies in incubation solution 2 for 48 hours at $4^{\circ} \mathrm{C}$ under gentle agitation. Then, the slices were washed several times in PBS prior secondary antibody incubation diluted in incubation solution 2 for two hours at room temperature. Coverslips were mounted on slides using Aqua Poly/Mount (Polysciences, Inc.) mounting medium.

\subsubsection{Imaging}

Confocal laser scanning micrographs of organotypic hippocampal slices and autaptic hippocampal cultures were acquired with a Leica TCS-SP5 confocal microscope equipped with a tuneable White Light Laser (WLL) excitation source and hybrid GaAsP Detectors (HyD). High-magnification single-plane micrographs (1024x1024; pixel spacing $x, y=50.6$ $\mathrm{nm}$ ) of randomly selected sites of synaptic neuropil were imaged in sequential scanning 
mode with an HCX PL APO 100x/1.44NA oil objective and a pinhole setting of $0.38 \mathrm{AU}$. Acquired images were exported from LAS AF (Leica) acquisition software as TIF files for quantitative analysis.

\subsubsection{Image processing and analysis}

Confocal images were subjected to spatial deconvolution by use of two ImageJ (National Institutes of Health; Bethesda, MD) plugins: point spread functions (PSF) were generated using Diffraction PSF 3D plugin and iterative deconvolution was performed with the Richardson-Lucy algorithm (DeconvolutionLab plugin; Biomedical Imaging Group, EPFL; Lausanne, Switzerland).

The Colocalization plugin of ImageJ was used to detect and graphically represent sites of colocalization between Synaptobrevin-1 or SNAP23 and vesicular glutamate transporter 1 (VGLUT1). The plugin overlays 8-bit images from green (Synaptobrevin-1) and red (VGLUT1) channels and displays colocalized points in white (display value $=255$ ). Two signals were considered colocalized if their respective channel intensities exceeded an arbitrary threshold (set to 40 for both channels) and the ratio of pixel intensities for both channels exceeded $50 \%$. Identical parameters were used for the analysis of control and mutant slices. The Analyse Particles feature of ImageJ was used to count and to measure the area of punctate labelling and sites of colocalization. To quantify and compare the degree to which Synaptobrevin-1 is spatially associated with glutamatergic presynapses in control and mutant slices the relative proportion of VGLUT1-positive presynapses exhibiting colocalization with Synaptorevin-1 was calculated by dividing the number of individual colocalized sites by the total number of VGLUT1 puncta. These approaches are potentially vulnerable to the following sources of inaccuracy: (i) the ImageJ analyse particles feature occasionally failed to recognize VGLUT1 puncta or sites of colocalization in close proximity to one another as independent signals and (ii) this type of analysis is insensitive to multiple sites of colocalization within a single VGLUT1 puncta. However, both genotypes were subject to the same experimental bias and analysed under identical conditions.

Statistical analyses were performed with GraphPad Prism (Version 6.00; GraphPad, LaJolla,CA). Normally distributed data sets were compared by unpaired Student t-tests. Images (27 images each from control and Synaptobrevin-2 KOs; 18 images each from control and SNAP25 KOs) from two animals per genotype were included in the analysis. 
2.6.

Electron Microscopy Techniques

\subsubsection{Materials}

\subsubsection{Chemicals and Reagents}

\begin{tabular}{|c|c|c|}
\hline Product & Company & Cat. No. \\
\hline 2-Dodecenylsuccinic acid anhydride (DDSA) & Serva & 20755 \\
\hline Acetone, glass distilled & Electron Microscopy Sciences & 10015 \\
\hline Bovine Serum Albumin & Sigma - Aldrich & A-3350 \\
\hline Chloroform & EMSURE & 1.024 .451 .000 \\
\hline copper 100 mesh grids & Plano & G2410C \\
\hline DMP-30 & Plano & R1065 \\
\hline Gelatin capsules & Electron Microscopy Sciences & 70100, Size 00 \\
\hline Glycid ether 100 & Serva & 21045 \\
\hline Gridboxes & Plano & B801003080 \\
\hline Hexadecane & Fluka & 52276 \\
\hline $\mathrm{KOH}$ & MERCK & UN1813 \\
\hline Lead(II)nitrate & MERCK & 1.073 .980 .100 \\
\hline Methylnadic anhydride (MNA) & Serva & 29452 \\
\hline Osmium tetroxide $\left(\mathrm{OsO}_{4}\right)$ & EMS & 19119 \\
\hline Protein A $10 \mathrm{~nm}$ gold & Utrecht & \\
\hline Sample holder half cylinder & Leica & 16770135 \\
\hline Sample holder middle plate & Leica & 16770136 \\
\hline Sodium Citrate Dihydrate & Calbiochem & 567446 \\
\hline Specimen carriers Type A, $3 \mathrm{~mm} \varnothing$ & Leica & 16770141 \\
\hline Specimen carriers Type B, $3 \mathrm{~mm} \varnothing$ & Leica & 16770142 \\
\hline Tannic Acid & Sigma-Aldrich & 403040 \\
\hline Uranyl acetate & SPI-CHEM & 2624 \\
\hline Vinylec E (Formvar®) Resin & Plano / TED Pella, inc. & $21201 / 19223$ \\
\hline
\end{tabular}

\subsubsection{Equipment}

\begin{tabular}{|l|l|}
\hline Product & Company \\
\hline JEM-2100 200kV & JEOL \\
\hline Leica EM AFS2 & Leica \\
\hline Leica EM HPM100 & Leica \\
\hline Leica Ultracut UCT ultramicrotome & Leica \\
\hline Leica-EM TRIM m. Stereo 2 & Leica \\
\hline Orius SC1000 CCD camera & GATAN \\
\hline Sharp:eye CCD camera & Tröndle, TRS \\
\hline Ultra 45॰ diamond knife & Diatome \\
\hline Zeiss LEO 912-Omega 80kV & Zeiss \\
\hline
\end{tabular}




\subsubsection{Media and buffer}

1\% Formvar: (Vinylec E Resin) in chloroform

$\underline{0.3 \% \text { lead citrate (aqueous) }}$

$1 \%$ uranyl acetate (aqueous)

$\underline{0.1 \% \text { Tannic acid in acetone }}$

$\underline{2 \% \mathrm{OsO}_{4}}$. in acetone

EPON Mixture: (21.4 g Glycidether, $14.4 \mathrm{~g}$ DDSA, $11.3 \mathrm{~g} M N A, 0.84 \mathrm{ml} D M P-30)$

\subsubsection{Methods}

\subsubsection{High-Pressure-Freezing}

Hippocampal slices were transferred with forceps by handling the small membrane confetti into a small petri dish of Culture Medium. Excess membrane was trimmed at the sides with a scalpel. The slice was dipped into external cryoprotectant (20\% bovine serum albumin in Culture Medium) and carefully loaded into the central cavity of the highpressure freezing sample carriers $(2 \mathrm{~mm} \varnothing, 0.1 \mathrm{~mm}$ height) with the membrane side facing upwards. Carriers were placed in the sample cartridge of the EM HPM100 (Leica, Vienna, Austria) High-Pressure Freezing device. The lid was dipped in 1-hexadecene and placed on top the sample carrier, in order to facilitate the removal of the lid after freezing. The sample carrier was then inserted into the high-pressure chamber for rapid cryofixation. Finally, the frozen sample in the specimen carriers was automatically released into the liquid nitrogen dewar and the specimen carriers were then transferred with pre-cooled forceps into cryotubes and stored in liquid nitrogen until further processing.

\subsubsection{Freeze Substitution and EPON embedding}

The high pressure frozen samples were freeze-substituted in the Leica EM AFS-2 unit (AFS: automated freeze substitution, Vienna, Austria) at low temperatures $\left(-90^{\circ} \mathrm{C}\right)$ according to the following protocol ('Paris protocol') modified from S. Marty (Rostaing et al., 2006; Siksou et al., 2007): $100 \mathrm{~h}$ in $0.1 \%$ tannic acid in dehydrated acetone at $-90^{\circ} \mathrm{C}$; four washing steps within $2 \mathrm{~h}$ in acetone at $-90^{\circ} \mathrm{C} ; 7 \mathrm{~h}$ fixing and contrasting in $2 \%$ osmium tetroxide $(\mathrm{OsO} 4)$ during a temperature ramp from $-90^{\circ} \mathrm{C}$ to $4^{\circ} \mathrm{C}$ within $40 \mathrm{~h}(14 \mathrm{~h}$ with $5^{\circ} \mathrm{C} / \mathrm{h}$ increments from -90 to $-20^{\circ} \mathrm{C}, 16 \mathrm{~h}$ at $-20^{\circ} \mathrm{C}, 2.4 \mathrm{~h}$ with $10^{\circ} \mathrm{C} / \mathrm{h}$ increments to $4^{\circ} \mathrm{C}$ ); 
three washing steps in acetone at $4^{\circ} \mathrm{C}$ for $4 \mathrm{~h}$; Infiltration with EPON resin was performed at room temperature in several steps: acetone/EPON 1:1 for $3 \mathrm{~h}$, overnight incubation in 90\% EPON in acetone at RT, $36 \mathrm{~h}$ incubation in pure EPON at RT. Finally, specimen carriers containing infiltrated samples were placed sample-side-up on Parafilm®-covered glass slides. EPON-filled gelatin capsules were inverted on the specimen carriers and polymerized for $24 \mathrm{~h}$ at $60^{\circ} \mathrm{C}$.

\subsubsection{Sectioning and contrasting}

Ultrathin sections $\left(60 \mathrm{~nm}\right.$ ) were cut with a diamond knife (Ultra $45^{\circ}$, Diatome) on a Leica Ultracut UCT ultramicrotome (Leica, Vienna, Austria). Sections were collected onto a Formvar-filmed, carbon-coated, copper mesh grid. Ultrathin sections were treated $30 \mathrm{~min}$ with $1 \% \mathrm{UA}$ in $\mathrm{ddH} 2 \mathrm{O}$, washed three times by dipping the grids seven times in $\mathrm{ddH} 2 \mathrm{O}$, treated 2 min with $0.3 \%$ lead citrate, washed again and dried with filter paper. For electron tomography, $200 \mathrm{~nm}$ thick sections were collected onto Formvar-filmed, carbon-coated copper mesh grids. To introduce fiducial markers for tomographic reconstruction, a 1:3 dilution of Protein A (ProtA) conjugated to $10 \mathrm{~nm}$ gold particles (Utrecht, The Netherlands) in $\mathrm{ddH}_{2} \mathrm{O}$ was applied to both sides of the grid for $1 \mathrm{~min}$ each. The grids were washed briefly in $\mathrm{ddH}_{2} \mathrm{O}$ and dried with filter paper.

\subsubsection{Imaging}

For two-dimensional ultrastructural analyses of synaptic morphology, electron micrographs from ultrathin sections were acquired with a transmission electron microscope (Zeiss LEO 912-Omega) operating at $80 \mathrm{kV}$. Micrographs (2048 x 2048 pixels) of synaptic profiles were acquired with a sharp:eye CCD camera (Tröndle, TRS) at 12,500 fold magnification and a pixel spacing of $0.95 \mathrm{~nm}$.

For the three-dimensional electron tomographic analysis of synaptic vesicle docking/priming, fiducially-coated $200 \mathrm{~nm}$-thick sections were imaged in a JEM-2100 transmission electron microscope (JEOL) operating at $200 \mathrm{kV}$. The SerialEM software enabled automated tilt series acquisition by using predictions for specimen positions during the tilt series based on the positions at previous tilts (Mastronarde, 2005). Singleaxis tilt series were recorded from -60 to $+60^{\circ}$ with $1^{\circ}$ increment binned by the factor two at 30,000 fold magnifications with an Orius SC1000 camera (Gatan). The target defocus 
during image acquisition was set to $-0.2-0.4 \mu \mathrm{m}$. The individual images in the tilt series before tomogram reconstruction had $1336 \times 1336$ pixels with an individual pixel spacing of $0.52 \mathrm{~nm}$.

\subsubsection{IMOD Software package}

All tomographic volumes were reconstructed from their respective tilt series with the IMOD package (Kremer et al., 1996; Mastronarde, 1997). Windows version required installation of a Unix-like environment called Cygwin. All IMOD programs use the MRC image file format ( $\left.{ }^{*} . m r c\right)$ and for an in-depth introduction and a tutorial to the IMOD software, the webpage of the Boulder laboratory for 3-D electron microscopy (http://bio3d.colorado.edu/) can be consulted. Tomogram generation was performed with the graphical user interface eTomo of the IMOD software package in a multi-step process. Afterwards, the *.mrc file extension was renamed in *.st to be recognized as single stack by eTomo. Second, in the pre-processing step, camera artefacts, like random x-rays causing extremely dark or bright pixels were removed by replacing them with neighbouring average values (program Ccderaser). In the following steps, the image stack was coarse aligned by using the programs Tiltxcorr, Xftoxg and Newstack. Then, a fiducial model was created computationally, based on the position of gold particles that were applied to both sides of the grid before image acquisition. These were then tracked through all projections by running the program Beadtrack. In the fine alignment step, the program Tiltalign was used to solve for displacements between the different tilted views to reduce the residual error mean. In a next step, three small sample tomograms (top, middle and bottom of the volume) were created to calculate the minimal volume of the final tomogram by defining the angle around the $x$ - and $y$-axes and the $z$-position of the section after drawing boundary lines at the end of biological material in the sample tomograms. Then, in a final alignment step by running the program Tomopitch, a fullaligned stack was produced using linear interpolation. During reconstruction, the projections were binned three times, resulting in a final isotropic voxel size of $1.55 \mathrm{~nm}$. Finally, the actual tomogram was computed (program Tilt) using a back projection algorithm and was trimmed and adjusted to the right contrast in a post-processing procedure. 


\subsubsection{Two-dimensional (2D) ultrastructural analysis of synaptic morphology}

Two-dimensional electron micrographs taken at 12,500 fold magnification were analyzed manually with the iTEM software version 5.1 (Olympus Soft Imaging Solutions $\mathrm{GmbH}$ ). The different parameters measured are depicted in Fig. 2.1 and comprised of the synaptic vesicle number, the presynaptic terminal area, the number of synaptic vesicles normalized to the terminal area (terminal density, Fig. 2.1 A), the synaptic vesicle cluster area, and the number of synaptic vesicles normalized to the cluster area (cluster density, Fig. 2.1. B). Moreover, the length of the PSD (Fig. 2.1 C), the number of recycling endosomes per presynaptic terminal (Fig. 2.1 D, open arrows) and the number of LDCVs (Fig. 2.1 D, white arrows) were quantified. Synaptic vesicle terminal density and cluster density are specified as number of synaptic vesicles per $0.01 \mu \mathrm{m}^{2}$ area. The PSD length is displayed in nanometers $(\mathrm{nm})$.
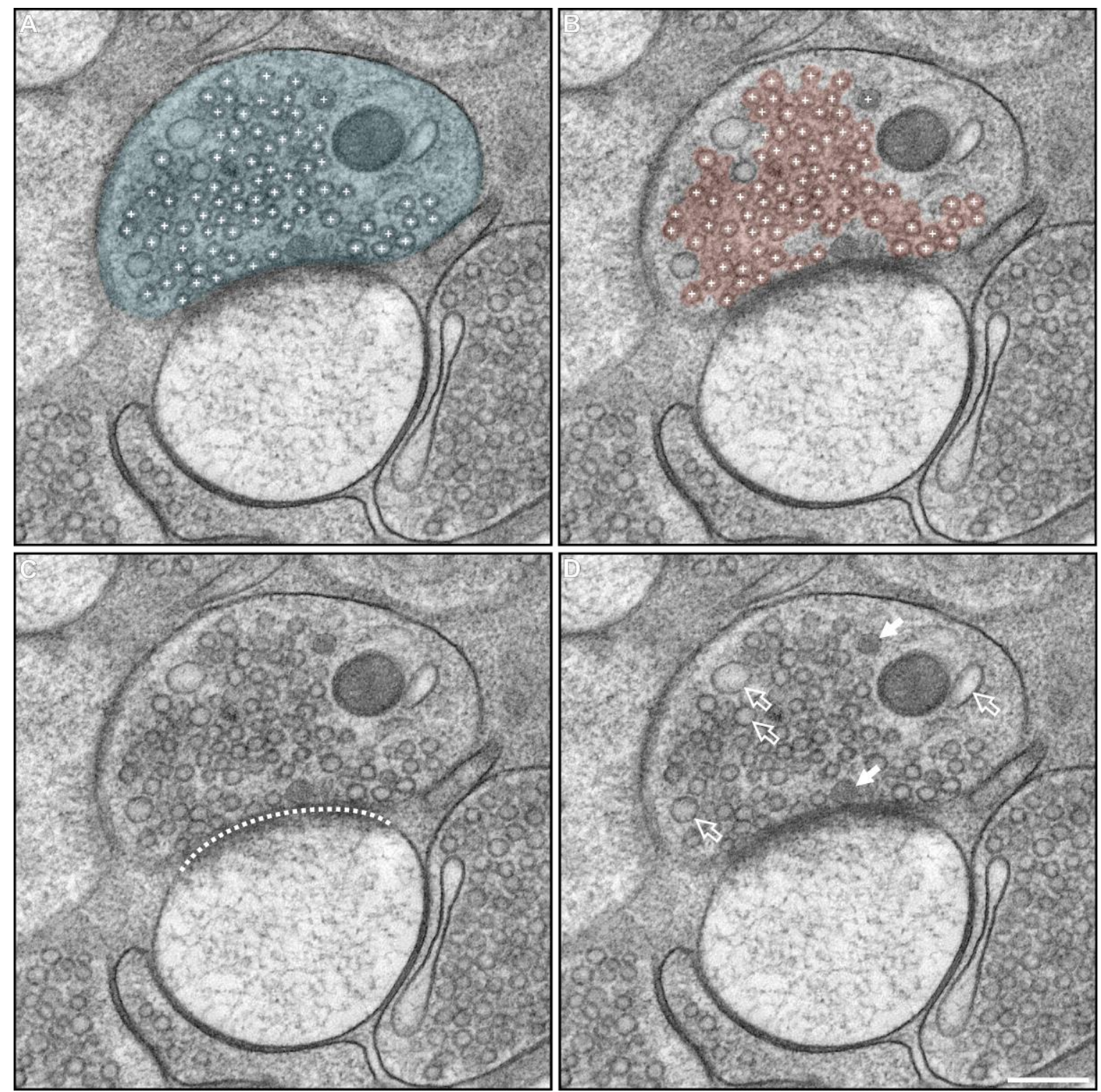
Figure 2.1. Two-dimensional ultrastructural analysis of synaptic morphology

Ultrastructural analysis of presynaptic parameters in electron micrographs from ultrathin sections. The number of synaptic vesicles (white crosses, A, B) within a presynaptic profile was normalized to the terminal area (blue, A) for the terminal density and to the cluster area (red, B) for the cluster density. The length of the postsynaptic density (white dotted line, C) was measured and the number of large dense core vesicles (LDCVs, white arrows, D) and endosomes (open arrows, D) was quantified. Scale Bar in D: $200 \mathrm{~nm}$.

\subsubsection{Three-dimensional (3D) electron tomographic (ET) analysis of synaptic vesicle docking}

Synaptic vesicle docking analyses were performed on tomographic volumes reconstructed with an isotropic voxel size of $1.55 \mathrm{~nm}$. Tomographic volumes were exported as TIFF image stacks (program tif2mrc) into ImageJ for quantitative analysis. The smallest vesicle distances from the outer leaflet of the synaptic vesicle membrane to the inner leaflet of the active zone membrane were measured using the straight line tool of the ImageJ software. Only synaptic vesicles observed to be in physical contact with the presynaptic membrane at their midline were considered docked (0-2 nm distance). Synaptic vesicles that were close to the active zone membrane, but not in physical contact were categorized together with the membrane-attached synaptic vesicles in a second 0-4 $\mathrm{nm}$ bin. Moreover, membrane proximal synaptic vesicles (0-40 nm distance) and all vesicles within $100 \mathrm{~nm}$ of the active zone were quantified. The active zone area was calculated by measuring the active zone length on each consecutive slice using the freehand line tool in ImageJ. The obtained values were added and then multiplied by the factor of $1.55 \mathrm{~nm}$ (isotropic voxel size; z-dimension) to obtain the final active zone area per tomogram. The final results for the quantifications are displayed as the number of vesicles in the respective bin $(0-2 \mathrm{~nm}$; 0-4 nm; 0-40 nm and 0-100 nm) normalized to $0.01 \mu^{2}$ active zone area. The mean synaptic vesicle diameter $(\mathrm{d}, \mathrm{nm})$ was calculated from the area $(\mathrm{A})$ of the vesicle measured at its midline to the outer leaflet of the synaptic vesicle membrane using the elliptical selection tool in Image $(d=2 \sqrt{ }(A / \pi))$. As the sample sections were exposed to high electron doses during the image series acquisition, sample shrinkage in the zdimension was commonly observed, resulting in deformed, compressed synaptic vesicles shapes. The mean synaptic vesicle volume $(\mathrm{V})$ was therefore extrapolated from the mean area measured at the midline of the synaptic vesicle and the presented values therefore include the vesicular phospholipid bilayer $\left(V=\pi d^{3} / 6\right)$.

For the images in this study, seven consecutive virtual tomographic slices were projected onto each other to be able to display a $10.88 \mathrm{~nm}$ thick sub-volume, using the Slicer tool of the $3 \mathrm{dmod}$ software of the IMOD software package. To illustrate the spatial 
arrangements of synaptic vesicles with respect to the active zone, ultrastructural features of presynaptic profiles observed in tomographic reconstructions were manually segmented using the $3 \mathrm{dmod}$ software of the IMOD software package. The active zone membrane was reconstructed by drawing a contour on each consecutive section of the tomogram. Synaptic vesicle, endosomal and LDCV membranes were not segmented by tracing the vesicle outlines, but rather represented schematically as individual closed contours that were placed around the center of the vesicles. 


\subsection{Statistical Analysis}

Statistical analysis was performed using Prism (version 5.0) software. To test sample distributions for their normality, the Kolmogorov-Smirnov test was used.

For the two-dimensional ultrastructural analysis of synaptic morphology the following parameters were obtained (for details refer to 2.6.2.6. Two-dimensional ultrastructural analysis of synaptic morphology): number of vesicles per synaptic profile, the synaptic vesicle terminal density, the synaptic vesicle cluster density, the average PSD length $(\mathrm{nm})$, the number of endosomes and the number of LDCVs per synaptic profile. Most of the data sets from the two-dimensional analysis deviated from being normally distributed and therefore for all these parameters the nonparametric MannWhitney $U$ test was used. Data presented in the text as mean and standard error of the mean $(S E M)$ : (mean \pm SEM), $N=$ number of animals, $n=$ number of synaptic profiles.

For the three-dimensional electron tomographic analyses the following parameters were measured (for details refer to 2.6.2.7. Three-dimensional electron tomographic analysis of synaptic vesicle docking): number of synaptic vesicles within $100 \mathrm{~nm}$ of the active zone normalized to $0.01 \mu^{2}$ active zone area, number of synaptic vesicles within $100 \mathrm{~nm}$ of the active zone normalized to $0.01 \mu \mathrm{m}^{2}$ active zone area, number of synaptic vesicles within $100 \mathrm{~nm}$ of the active zone normalized to $0.01 \mu \mathrm{m}^{2}$ active zone area, number of synaptic vesicles within $100 \mathrm{~nm}$ of the active zone normalized to $0.01 \mu \mathrm{m}^{2}$ active zone area, mean outer synaptic vesicle diameter and mean synaptic vesicle volume. Most of these data sets were normally distributed and were therefore compared by using a parametric unpaired student's t-test. For genotypes exhibiting dramatic reductions in the number of docked vesicles, which resulted in non-Gaussian distributions, the non-parametric Mann-Whitney $U$ test was used. Data presented in the text: (mean \pm SEM), $N=$ number of animals, $n=$ number of tomograms.

The data obtained from electrophysiological experiments in order to access the physiological consequences of Cre-mediated Munc13-1 deletion in neurons, the following parameters were compared between the two groups: EPSC amplitude (nA), RRP size (nC) measured during hypertonic sucrose solution application and Pvr (\%). For all three parameters, the non-parametric Mann-Whitney $U$ test was used. In the control group (pFUG-GFP infected), one cell was excluded from the analysis since the measured RRP size was 8 fold higher than the calculated mean of the data set. Data presented in the text: (mean $\pm \mathrm{SEM}$ ), $\mathrm{N}=$ number of cultures, $n=$ cells. 


\section{Results}

3.1. Analysis of the morphological correlate of synaptic vesicle priming

\subsubsection{Ultrastructural organization of mouse hippocampal organotypic slice cultures}

The experimental approach used in the present study yielded excellent ultrastructural preservation of high-pressure frozen and freeze-substituted hippocampal organotypic slices. To effectively freeze slices without subjecting them to hyperosmotic shock, different external cryoprotectants were tested for their ability to inhibit ice-crystal formation (data not shown). Freezing of the samples in cell culture medium resulted in sub-optimal freezing quality. The best freezing quality throughout the whole sample was obtained with a previously published combination of $20 \%$ dextran and $5 \%$ sucrose in culture medium, however the osmolarity of this solution measured $\sim 600$ mOsm, which was in our opinion too high to guarantee physiological conditions during freezing (Fernández-Busnadiego et al., 2010b; Zuber et al., 2005). A solution of $20 \%$ BSA in culture medium was ultimately selected for the study, since it yielded excellent preservation of synaptic ultrastructure in large areas while exposing the sample to only a minor increase in osmolarity (to $~ 340$ mOsm).

After cryo-immobilization, well conserved samples were characterized by a densely packed neuropil of neuronal processes with smooth plasma membranes exhibiting parallel bilayers and small, round and symmetrically shaped synaptic vesicles (Fig. 3.1). Excitatory (asymmetric, Gray's Type I), glutamatergic spine synapses were identified by their prominent postsynaptic density (PSD) in the postsynaptic spine head and by an accumulation of synaptic vesicles in close proximity to the active zone of the presynaptic terminal (Fig. 3.1 A, B). Spine synapses lacked mitochondria and microtubules in their postsynaptic compartments. Some spine heads contained tubular organelles bearing morphological characteristics consistent with those described for the spine apparatus. The spine apparatus is thought to function in postsynaptic $\mathrm{Ca}^{2+}$-buffering by serving as an intracellular $\mathrm{Ca}^{2+}$-storage, and to participate in postsynaptic plasticity processes (Fig. 3.1 B) (reviewed in Segal et al. 2010). Occasionally, we observed astrocytic processes in the vicinity of a synaptic cleft, which could be identified by the presence of small electrondense glycogen particles (Fig. 3.1 A). Astrocytes are known to provide trophic support to neurons and have been implicated in glutamate recycling at synapses, both being important functions in synaptic maintenance. 

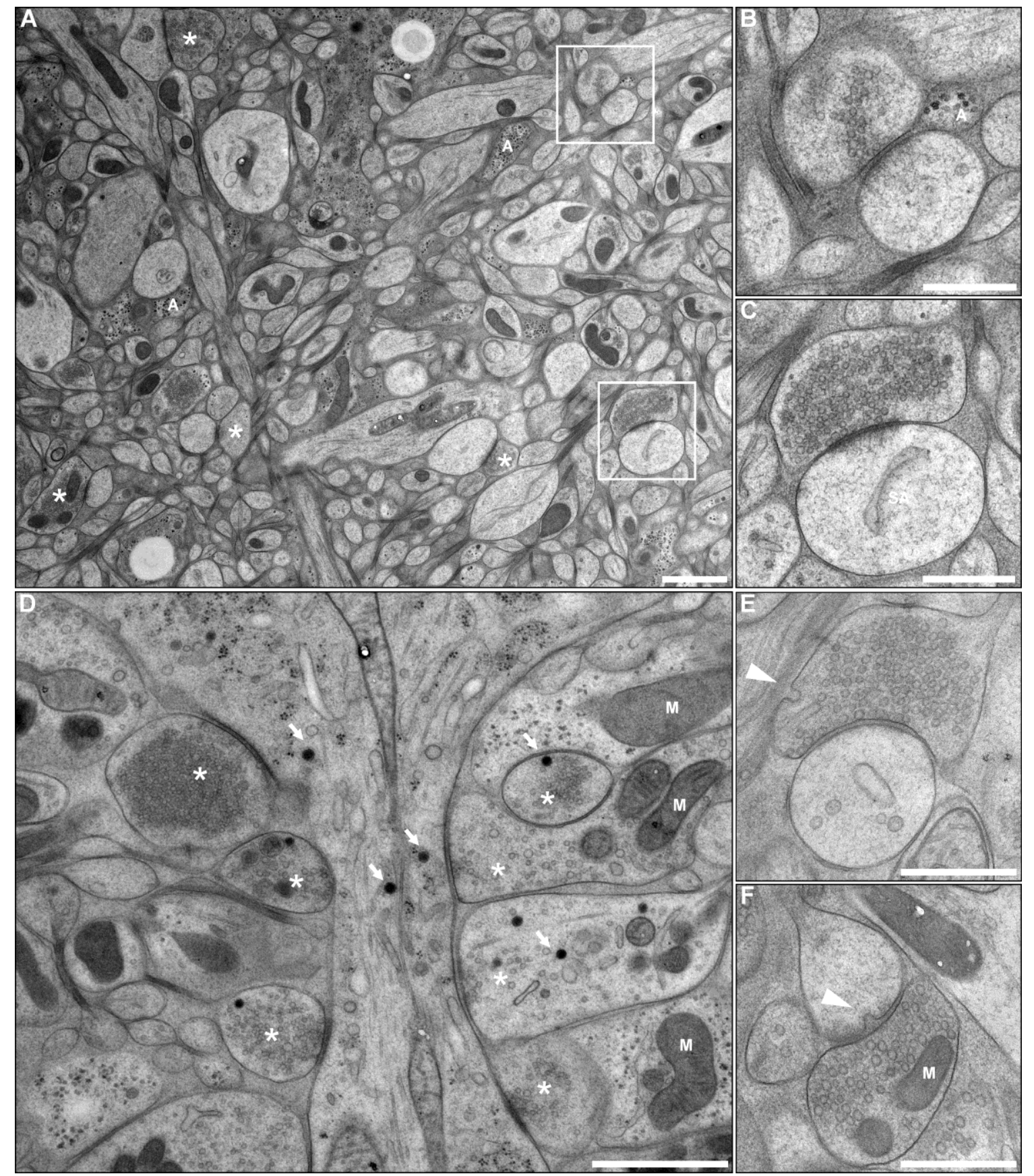

Figure 3.1. Ultrastructural organization of mouse hippocampal organotypic slice cultures.

Electron microscopy (EM) samples prepared by high-pressure freezing fixation and freeze substitution exhibit excellent ultrastructural preservation of neuropil in organotypic hippocampal slices (A-F). Glutamatergic, excitatory synapses (Gray's Type I; asterisks in A) exhibit a prominent postsynaptic density (PSD) and an accumulation of synaptic vesicles at the presynaptic active zone. Glutamatergic spine synapses are characterized by a small postsynaptic compartment lacking mitochondria (B, C; enlarged from panel A). Dendritic spines occasionally contain a spine apparatus ("SA" in C). Astrocyte processes contacting synapses can be identified by their glycogen particle content ("A" in A and B). Inhibitory synapses (Gray's Type II) preferentially contact the cell somata and dendritic shafts and can be identified by an absence of a prominent postsynaptic density (asterisks in D). In comparison to small synaptic vesicles, large dense core vesicles (LDCVs) are characterized by a larger size and an electron-dense appearance (white arrows in D). Well-preserved samples exhibit unswollen mitochondria with regular cristae ( $M$ in $\mathbf{D}$ and $\mathbf{F}$ ). Rapid cryo-fixation for EM can capture pre- and postsynaptic clathrin-mediated endocytic events (white arrowheads; E, F). Scale Bar in A: $1 \mu \mathrm{m}$; B, C, E, F: $500 \mathrm{~nm}$; D: $2 \mu \mathrm{m}$. 
Synapses contacting dendritic shafts or neuronal cell bodies often displayed a weaker postsynaptic density (symmetric, Gray's Type II) and were most likely of an inhibitory type (Fig. 3.1 D). A consequence of maintaining a near-native state of ultrastructural preservation in high-pressure frozen samples is that classical criteria for discriminating synaptic subtypes in conventionally prepared EM samples are less evident. Indeed, differences in vesicular shape and size, induced by the differential tonicity of excitatory and inhibitory vesicular populations upon chemical fixation, are no longer apparent in rapidly frozen material (Gaugler et al., 2012; Gray, 1959; Kaeser et al., 2009; Megías et al., 2001; Siksou et al., 2013). Rapid high-pressure freezing fixation of unstimulated organotypic slices could capture dynamic cellular events like pre-synaptic clathrindependent endocytosis as well as postsynaptic receptor endo- or exocytosis at the PSD (Fig. 3.1 E, F). The preservation of cytoskeletal components by the applied freeze substitution protocol in slices frozen under physiological conditions was indicated by the presence of clathrin molecules coating endocytic intermediates. Slices exhibiting artifacts from suboptimal freezing conditions (e.g. nuclear chromatin reticulation resulting from ice crystal formation, pressure induced membrane disruption) were excluded from my analysis.

\subsubsection{Synaptic vesicle interactions with the active zone membrane}

In this study, I use the terms "active zone" or "release sites" to describe the presynaptic plasma membrane in direct apposition to the PSD. Due to the fact that I did not image full synaptic terminals, but rather synaptic sub-volumes with a focus on the active zone region, I use the term "synaptic profiles", which refers to the sub-volume of the presynaptic terminal sampled in a respective tomogram. The definitions for "tethers", describing filaments from synaptic vesicles to the active zone, and "connectors", referring to filaments between synaptic vesicles, were taken from cryo-electron tomographic studies by $R$. Fernández-Busnadiego and colleagues (Fernández-Busnadiego et al., 2010, 2013). The number of tethers and connectors in tomographic reconstructions was not quantified in the course of the present study. Morphologically docked (or simply "docked") synaptic vesicles describe vesicles in direct membrane to membrane contact at the active zone (Fig.3.2 A1, A2). Such vesicles would fall into the $0-2 \mathrm{~nm}$ distance from the active zone, since the maximum resolution achievable in the analysed tomographic reconstructions was limited by an isotropic voxel size of $\sim 1.6 \mathrm{~nm}$. The term "membrane-attachment" is used as a synonym for the term "docking" in this study. The term "closely tethered 
synaptic vesicles" describes all vesicles within 0-4 $\mathrm{nm}$ of the active zone, including those that were membrane-attached and those that were potentially linked by multiple short filaments to the release site (Fig. 3.2 B1, B2, tethers indicated by white arrow heads). Multiple short tethers ( $<5 \mathrm{~nm}$ length) seen in cryo-electron tomograms were previously described as likely corresponding to assembled SNARE complexes (FernándezBusnadiego et al., 2010). Occasionally, potential synaptic vesicle hemifusion events were detected at active and periactive zone sites (Fig. 3.2 A2). These vesicles were included in the category of membrane-attached synaptic vesicles $(0-2 \mathrm{~nm})$ in my analysis. Very rarely, I was able to see an open fusion pore during synaptic vesicle fusion (Fig. 3.2. C1) or observe full collapse fusion events (Fig. 3.2. C2). These vesicles were excluded from the analysis. Occasionally, I observed inward membrane curvatures at periactive zonal sites that were considered to be early endocytotic events (Fig. 3.2 D1, D2).

The term "membrane-proximal synaptic vesicles" was used to describe synaptic vesicles within $40 \mathrm{~nm}$ of the active zone membrane. These vesicles would have been included in the pool of membrane-attached synaptic vesicles in studies that considered all vesicles within a diameter distance (e.g. $40 \mathrm{~nm}$ ) as "docked" (discussed in: Verhage \& Sørensen 2008). Moreover, recent studies showed that synaptic vesicles within $45 \mathrm{~nm}$ of the active zone can still connect to the plasma membrane via single long tethers (FernándezBusnadiego et al., 2010, 2013; Siksou et al., 2009, 2011).
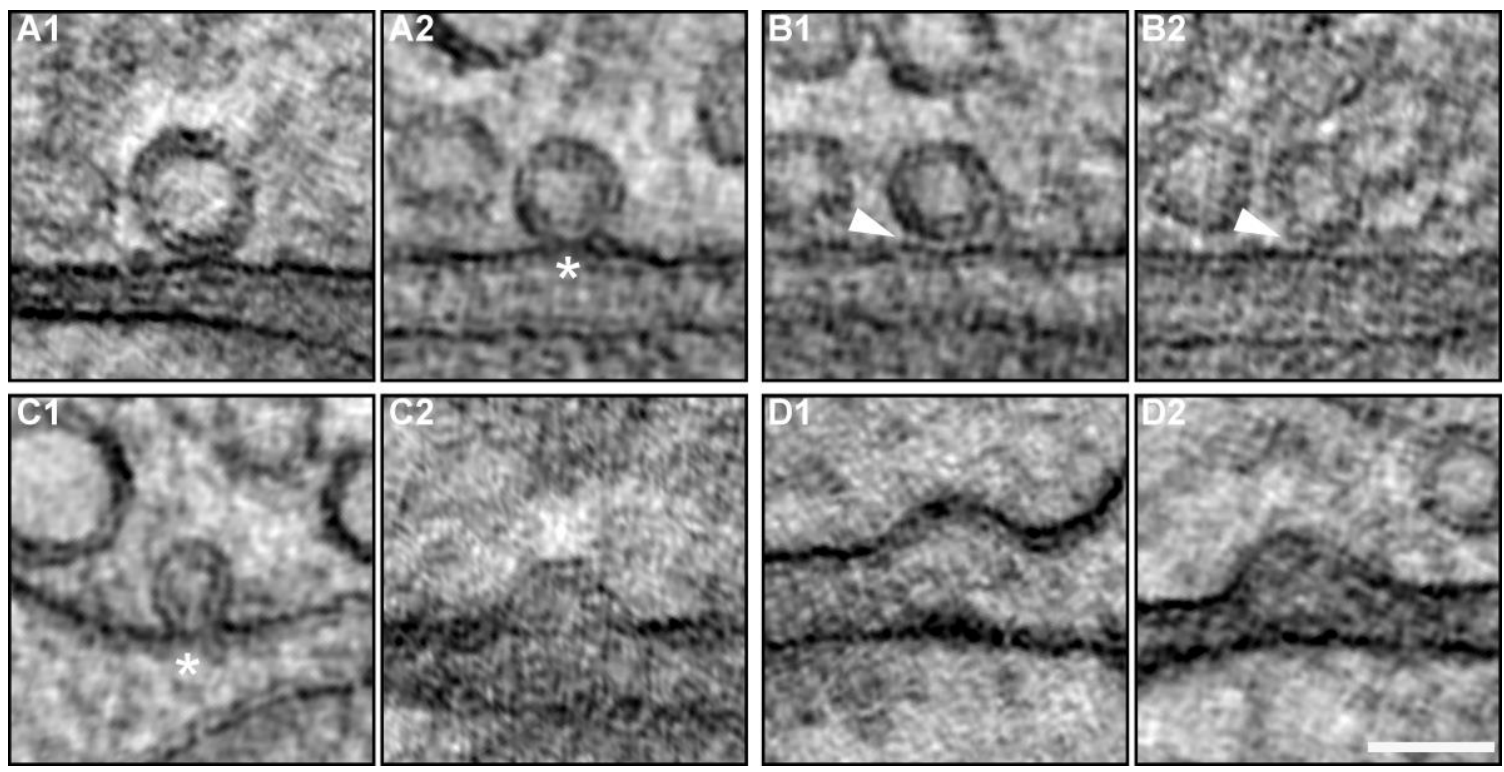

Figure 3.2. Direct interactions of synaptic vesicles with the active zone membrane

A Direct membrane-attachment of synaptic vesicles (SVs) with the active zone membrane $(0-2$ $\mathrm{nm})$. A1 No visible gap between the inner phospholipid layer of the active zone plasma membrane and the outer vesicular phospholipid layer. A2 Occasional inward curvature of the active zone membranes towards the membrane-attached SV displaying potential early hemifusion events (asterisk). B (B1+B2) Synaptic vesicles are not in direct contact, but closely tethered to the 
membrane (0-4 nm) by multiple, short filaments (white arrowheads). C Synaptic vesicle fusion events. C1 A synaptic vesicle is in a fused state displaying a prominent open fusion pore (asterisk). C2 Full collapse fusion event at the active zone membrane. D (D1+D2) Presynaptic endocytotic events at the periactive zone. Scale bar in D2: $50 \mathrm{~nm}$.

\subsubsection{Docking Analysis of presynaptic mutants}

\subsubsection{The role of the synaptic vesicle priming factors Munc13-1 and Munc13-2 in synaptic vesicle docking}

Munc13 proteins are key regulators of synaptic vesicle release, as evidenced by the complete loss of evoked and spontaneous neurotransmitter release in the absence of Munc13 priming factors. Munc13s reside in the dense protein network at the presynaptic active zone. Their mode of action is believed to involve the opening of the t-SNARE Syntaxin-1 by displacing Munc18-1, allowing t-SNARE acceptor complex formation (Ma et al., 2011). A previous study has shown that $\mathrm{KO}$ of Munc13 proteins results in a massive decrease in the number of "docked" or "membrane-attached" synaptic vesicles in neurons (Siksou et al., 2009). Therefore I decided to start with the reanalysis of synaptic vesicle docking in Munc13-1/2 DKO neurons in order to validate my experimental system (Augustin et al., 1999a; Varoqueaux et al., 2002). Homozygous Munc13-2 KO neurons are phenotypically indistinguishable from wild-type neurons (Varoqueaux et al., 2002). In this study, I therefore compared four Munc13-1/2 DKO animals $(\mathrm{N}=4)$ with four littermate control animals $(\mathrm{N}=4)$ from two cultures. The control animals were either wild-type or heterozygous for the Munc13-1 KO allele in a homozygous Munc13-2 KO background, or heterozygous for Munc13-1 and Munc13-2 KO alleles.

\subsection{D-EM analysis of synaptic morphology in Munc13-1/2 DKO neurons}

In a first experiment, ultrastructural parameters of Munc13-1/2 DKO synapses were analyzed in electron micrographs from $60 \mathrm{~nm}$ ultrathin EPON sections after high-pressure freezing fixation, freeze-substitution and EPON embedding in order to rule out any major changes of presynaptic morphology that could influence the results obtained from a highresolution electron tomographic analysis (Fig. 3.3). I did not observe any major morphological changes between control and Munc13-1/2 DKO synaptic profiles, although a potential increase in the size of synaptic vesicles was apparent in Munc13-1/2 DKO 
terminals (Fig. 3.3 A, B). I found that the total number of synaptic vesicles per synaptic profiles in Munc13-1/2 DKO synapses was unchanged in comparison to control synaptic profiles (control: $61.44 \pm 3.195, n=106$; Munc13-1/2 DKO: $70.53 \pm 4.203, n=103$ / $\mathrm{P}=0.2565$, n.s.; Fig. $3.3 \mathrm{C}$ ). Moreover, I did not detect any significant changes in the number of synaptic vesicles normalized to the presynaptic terminal size (synaptic vesicles per $0.01 \mu^{2}$ terminal area or synaptic vesicle terminal density) (control: $1.633 \pm 0.059$, $\mathrm{n}=106$; Munc13-1/2 DKO: $1.489 \pm 0.057, \mathrm{n}=103 / \mathrm{P}=0.0558$, n.s.) nor in the measured length of the PSD (control: $371.1 \pm 11.95, n=106$; Munc13-1/2 DKO: $393.7 \pm 12.97, n=103$ / $P=0.1895$, n.s.; Fig.3.3 D, F). However, I did observe a small decrease in the number of synaptic vesicles normalized to the size of the synaptic vesicle cluster (synaptic vesicles per $0.01 \mu \mathrm{m}^{2}$ cluster area or synaptic vesicle cluster density) (control: $3.149 \pm 0.056$, $\mathrm{n}=106$; Munc13-1/2 DKO: $2.883 \pm 0.055, \mathrm{n}=103$ / P=0.0018, **; Fig. $3.3 \mathrm{E})$. I also noticed an increase in the number of vesicular structures with a larger diameter than synaptic vesicles, possibly representing components of the endocytotic recycling pathway in the presynaptic terminal (control: $1.000 \pm 0.127, n=106$; Munc13-1/2 DKO: $1.650 \pm 0.198$, $\mathrm{n}=103$ / $\mathrm{P}=0.0462$, *; Fig. $3.3 \mathrm{G}$ ). A recent study indicated a role of proteins from the Munc13 family in LDCV release in neurons, without detecting any changes in the number of LDCVs in presynaptic terminals from Munc13-1/2 DKO and control neurons (van de Bospoort et al., 2012). I could confirm these results in our experimental setting as I failed to observe a difference in the number of LDCVs per synaptic profile (control: $0.236 \pm$ 0.056, n=106; Munc13-1/2 DKO: $0.233 \pm 0.054, n=103$ / P=0.8375, n.s.; Fig. 3.3 H).

\subsection{D-ET analysis of synaptic vesicle docking in Munc13-1/2 DKO neurons}

I then moved on to analyze synaptic vesicle docking in presynaptic terminals from electron tomographic reconstructions of $200 \mathrm{~nm}$-thick semithin sections through glutamatergic, spine synapses of control and Munc13-1/2 DKO samples (Fig. 3.4 A-F). For the threedimensional analysis of electron tomograms, I compared samples from three Munc13-1/2 DKO animals $(\mathrm{N}=3)$ with samples from two littermate controls $(\mathrm{N}=2)$. Control animals were either wild-type for the Munc13-1 KO allele in a homozygous Munc13-2 KO background, or heterozygous for both Munc13-1 and Munc13-2 KO alleles. In agreement with previous publications, I detected an almost complete loss of membrane-attached vesicles $(0-2 \mathrm{~nm})$ 


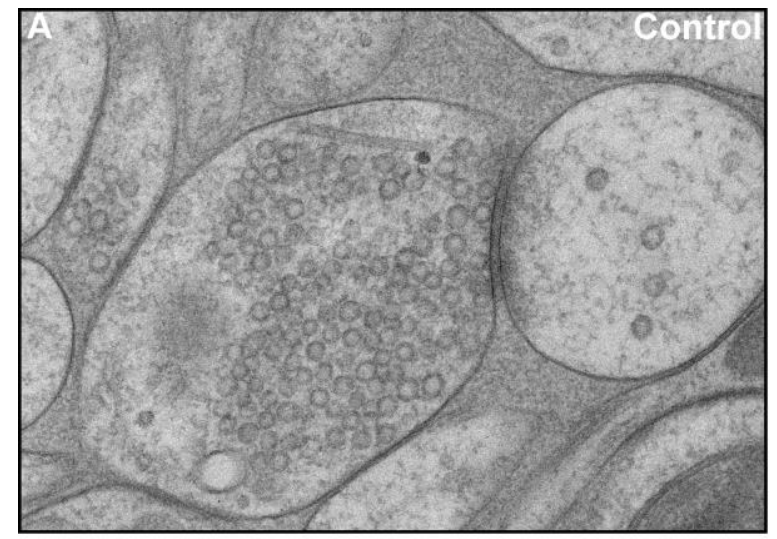

C

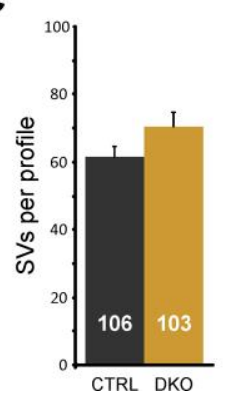

D

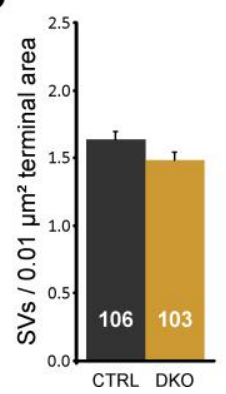

$\mathbf{E}$

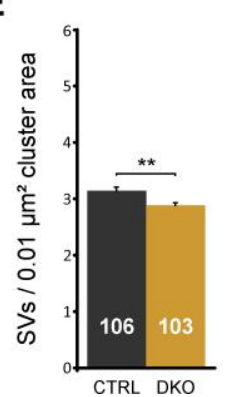

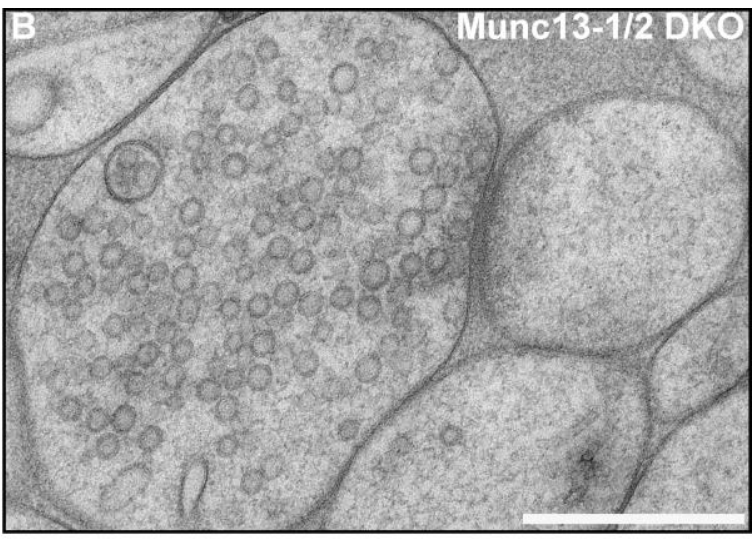

$\mathbf{F}$

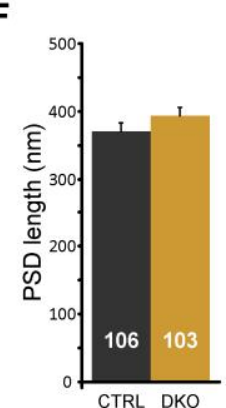

G

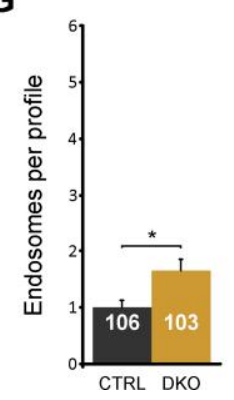

H

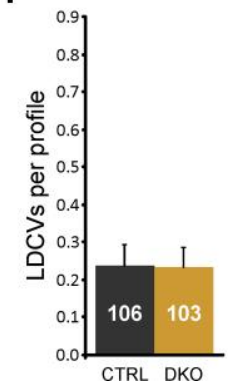

Figure 3.3. Two-dimensional ultrastructural analysis of synaptic morphology in Munc13-1/2 DKO neurons

Electron micrographs of control (A) and Munc13-1/2 DKO (B) synaptic profiles acquired from 60 $\mathrm{nm}$-thick ultrathin sections. Mean number of synaptic vesicles (SVs) per synaptic profile (C). Mean number of SVs normalized to synaptic terminal area (SV terminal density; D). Mean number of SVs normalized to SV cluster area (SV cluster density; E). Mean postsynaptic density (PSD) length (F) Mean number of endosomes per synaptic profile (G). Mean number of large dense-core vesicles (LDCVs) per synaptic profile (H). C-H: Control: N=4, $\mathrm{n}=106$; Munc13-1/2 DKO: N=4, $\mathrm{n}=103$ (Mean + SEM), $P<0.001:{ }^{* * *} ; \mathrm{P}<0.01:^{* *} ; \mathrm{P}<0.05:{ }^{*}$. Scale bar: B, $500 \mathrm{~nm}$.

normalized to $0.01 \mu^{2}$ active zone area in Munc13-1/2 DKO in comparison to control synaptic profiles (control: $0.979 \pm 0.134, \mathrm{n}=16$; Munc13-1/2 DKO: $0.042 \pm 0.004, \mathrm{n}=15$ / $\mathrm{P}<0.001,{ }^{* *}$; Fig. $3.4 \mathrm{I}$ ), which was still statistically significant after including vesicles that are closely tethered (0-4 $\mathrm{nm}$ ) normalized to $0.01 \mu \mathrm{m}^{2}$ active zone area (control: $1.651 \pm$ 0.201, $\mathrm{n}=16$; Munc13-1/2 DKO: $0.145 \pm 0.089, \mathrm{n}=15 / \mathrm{P}<0.001$, ${ }^{* * *}$; Fig. $\left.3.4 \mathrm{~J}\right) .14$ out of 15 synaptic profiles (93\%) harbored no membrane-attached $(0-2 \mathrm{~nm})$ vesicles, and 12 synaptic profiles (80\%) lacked docked and closely tethered vesicles (0-4 nm). The number of membrane-attached $(0-2 \mathrm{~nm})$ and closely tethered $(0-4 \mathrm{~nm})$ synaptic vesicles was reduced to $4 \%$ and $11 \%$ of control profiles, respectively. However, the number of vesicles that were close to the active zone (within $40 \mathrm{~nm}$ ) normalized to $0.01 \mu \mathrm{m}^{2}$ active zone area was unchanged (control: $2.487 \pm 0.188, \mathrm{n}=16$; Munc13-1/2 DKO: $2.113 \pm 0.179, \mathrm{n}=15$ / $\mathrm{P}=0.1626$, n.s.; Fig. $3.4 \mathrm{H}$ ). Consistent with this finding, the synaptic vesicles in Munc13$1 / 2$ DKO synapses seem to accumulate at a close distance to the active zone membrane, with a peak in the vesicle distribution around 8-10 nm (Fig. 3.4 M). Those membrane- 
proximal, but not docked vesicles, were often linked to the presynaptic plasma membrane by thin, long filaments similar to previous findings (Siksou et al., 2009). I also detected a decrease in the number of vesicles within $100 \mathrm{~nm}$ distance from the active zone membrane, normalized to $0.01 \mu^{2}$ active zone area (control: $6.547 \pm 0.395, n=16$; Munc13-1/2 DKO: $5.023 \pm 0.384, n=15$ / $P=0.0099$, ${ }^{* *}$; Fig. 3.4 G). A likely explanation for this is a dramatic increase in the mean outer synaptic vesicle diameter, first observed in two-dimensional electron micrographs from Munc13-1/2 DKO synapses, and subsequently confirmed by three-dimensional electron tomographic analysis (control: $46.25 \pm 0.485, \mathrm{n}=16$; Munc13-1/2 DKO: $50.15 \pm 0.715, \mathrm{n}=15 ; \mathrm{P}<0.001$, ${ }^{* \star *}$; Fig. $3.4 \mathrm{~K}, \mathrm{~N}$ ). This increase in the outer vesicle diameter in Munc13-1/2 DKO presynaptic terminals leads to a corresponding $28 \%$ increase in the synaptic vesicle volume (control: $52473 \pm$ 1656, $n=16$; Munc13-1/2 DKO: $67158 \pm 2853, n=15 ; P<0.001$, ***; Fig. 3.4 L). The increased number of larger vesicular structures, possibly components of the endocytotic recycling pathway or precursors of vesicular biosynthesis, in the presynaptic terminals in Munc13-1/2 DKO synaptic profiles raises the possibility that synaptic trafficking or vesicle recycling is disrupted in the absence of Munc13 proteins.

In summary, the ultrastructural findings presented here are in agreement with previously published data, showing an almost complete loss of membrane-associated synaptic vesicles in synapses lacking synaptic vesicle priming molecules of the Munc13 family (Siksou et al., 2009). Moreover, the inability of synaptic vesicles to dock to the presynaptic active zone membrane strongly supports the hypothesis that the terms morphological synaptic vesicle "docking" and physiological synaptic vesicle "priming" may describe the same molecular process.

\subsubsection{The role of the synaptic vesicle priming factors CAPS-1 and CAPS-2 in synaptic vesicle docking}

Next, I decided to focus on CAPS-1/2 proteins, which are thought to regulate synaptic vesicle priming in concert with Munc13s. Autaptic hippocampal cultures from CAPS1/2 DKO neurons revealed a complex physiological phenotype: similar to Munc13-1/2 DKO neurons, $39 \%$ of all CAPS-1/2 DKO neurons lack any presynaptic activity and are deficient of fusion-competent vesicles (Jockusch et al., 2007). However, in the remaining $61 \%$ cells, synaptic responses are not completely abolished, but EPSC sizes and the RRP probed by sucrose application are reduced by $68 \%$ and $85 \%$, respectively (Jockusch et al., 2007). 

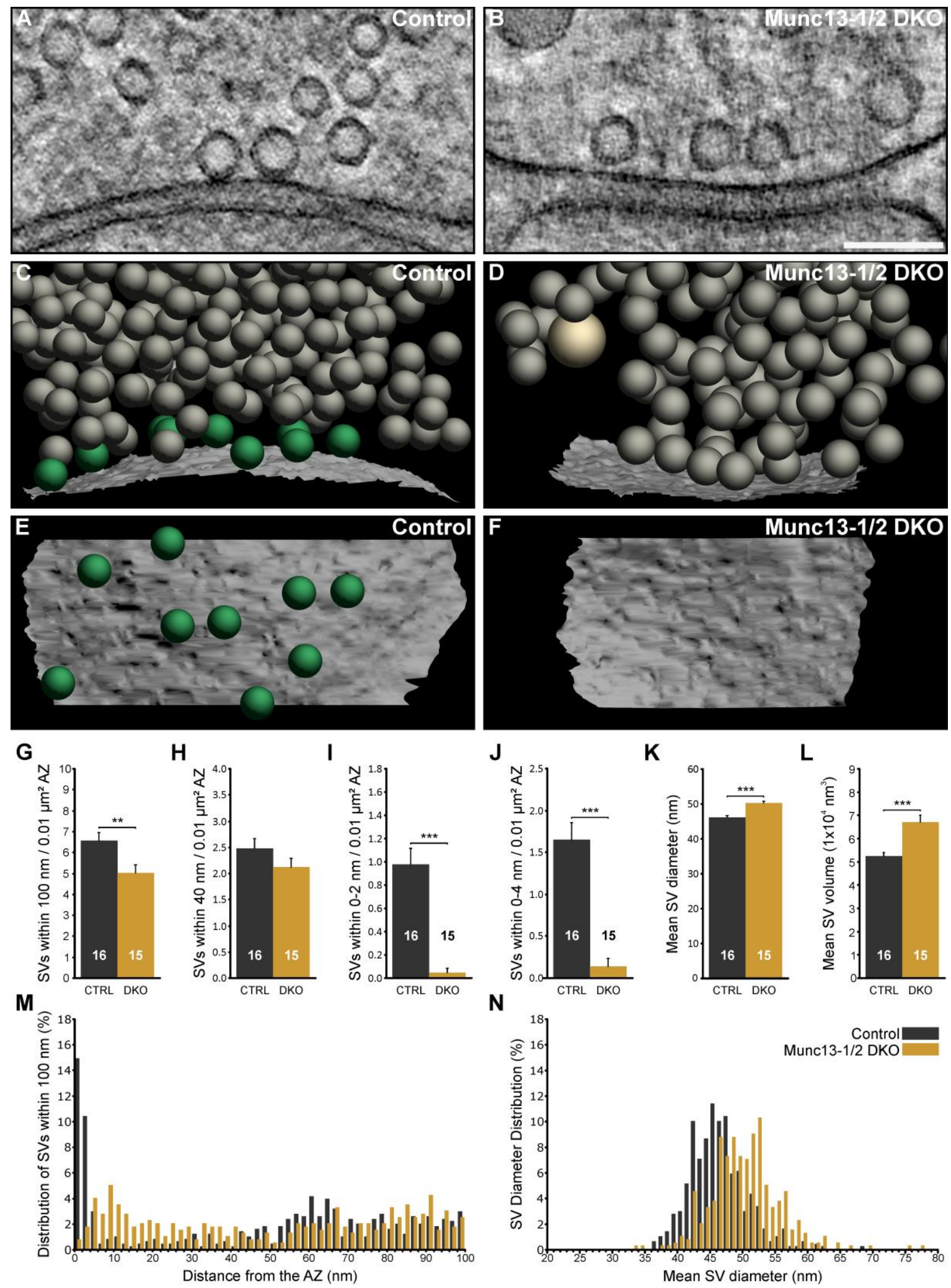

Figure 3.4. Three-dimensional electron tomographic analysis of synaptic vesicle docking in Munc13-1/2 DKO neurons

Tomographically reconstructed subvolumes of control (A) and Munc13-1/2 DKO (B) synapses from $200 \mathrm{~nm}$-thick sections. Three-dimensional models of tomographically reconstructed control (C) and Munc13-1/2 DKO (D) synaptic profiles. Ultrastructural features reconstructed in the models include the active zone plasma membrane (white), synaptic vesicles (membrane-attached, green; non- 
attached, grey), and large dense core vesicles (LDCVs; beige). Orthogonal views of control (E) and Munc13-1/2 DKO (F) tomographic models displaying the spatial arrangement of membraneattached synaptic vesicles within the reconstructed active zone area. Mean number of SVs within $100 \mathrm{~nm}$ of the $A Z$ normalized to $A Z$ area $(\mathbf{G})$. Mean number of SVs within $40 \mathrm{~nm}$ of the $A Z$ normalized to $A Z$ area $(\mathbf{H})$. Mean number of membrane-attached SVs (within 0-2 $\mathrm{nm}$ of the $A Z$ ) normalized to $A Z$ area (I). Mean number of membrane-attached and closely-tethered SVs (within 0$4 \mathrm{~nm}$ of the $A Z$ ) normalized to $A Z$ area $(\mathbf{J})$. Mean outer SV diameter (K). Mean SV volume including the membrane bilayer (L). Spatial distribution of SVs within a $100 \mathrm{~nm}$ distance of the $A Z$ membrane (M). Distribution of synaptic vesicle diameters of vesicles within $100 \mathrm{~nm}$ of the $A Z(\mathbf{N})$. G-L: Control: $\mathrm{N}=2, \mathrm{n}=16 ;$ Munc13-1/2 DKO: $\mathrm{N}=3, \mathrm{n}=15$ (Mean + SEM), $\mathrm{P}<0.001$ : ${ }^{* *} ; \mathrm{P}<0.01$ : **; $\mathrm{P}<0.05:{ }^{*}$. M, N: (511 SVs in control and $402 \mathrm{SVs}$ in Munc13-1/2 DKO synaptic profiles). Scale bar: $\mathbf{B}, 100 \mathrm{~nm}$.

As previously shown, homozygous CAPS-2 KO neurons are phenotypically indistinguishable from wild-type neurons (Jockusch et al., 2007). For the two-dimensional analysis of electron micrographs from ultrathin sections, four CAPS-1/2 DKO animals $(\mathrm{N}=4)$ were compared with three littermate control animals $(\mathrm{N}=3)$ from three cultures. The control animals were either wild-type or heterozygous for the CAPS-1 KO allele in a homozygous CAPS-2 KO background.

\subsection{D-EM analysis of synaptic morphology in CAPS-1/2 DKO neurons}

Morphologically, no major differences in the gross synaptic ultrastructure of neurons lacking both CAPS isoforms were observed in electron micrographs from ultrathin $(60 \mathrm{~nm})$ sections (Fig. 3.5 A, B). The total number of synaptic vesicles per synaptic profile did not differ in CAPS-1/2 deficient neurons in comparison to control samples (control: $63.15 \pm$ 3.641, $\mathrm{n}=109$; CAPS-1/2 DKO: $60.74 \pm 2.907, \mathrm{n}=115$ / $\mathrm{P}=0.9228$, n.s.; Fig. $3.5 \mathrm{C}$ ). Moreover, the synaptic vesicle terminal density was not significantly altered (control: 1.454 $\pm 0.059, n=109$; CAPS-1/2 DKO: $1.535 \pm 0.057, n=115$ / $P=0.2858$, n.s.; Fig. 3.5 D), with only a slight decrease in the synaptic vesicle cluster density (control: $3.436 \pm 0.061$, $\mathrm{n}=109$; CAPS-1/2 DKO: $3.285 \pm 0.056, \mathrm{n}=115 / \mathrm{P}=0.0308$, *; Fig $3.5 \mathrm{E})$ and no changes in the mean PSD length (control: $330.5 \pm 11.06, n=109$; CAPS-1/2 DKO: $315.2 \pm 8.849$, $\mathrm{n}=115$ / $\mathrm{P}=0.5539$, n.s.; Fig. $3.5 \mathrm{~F}$ ). In contrast to synapses lacking Munc13s, I did not observe an increase in the number of recycling endosomes per synaptic profile in neurons deficient for CAPS-1 and -2 in comparison to control neurons (control: $1.248 \pm 0.170$, $\mathrm{n}=109$; CAPS-1/2 DKO: $1.270 \pm 0.142, \mathrm{n}=115$ / $\mathrm{P}=0.5173$, n.s.; Fig.3.5 G). Also, no change in the number of LDCVs in synapses lacking all CAPS isoforms was detected in my experimental setting (control: $0.147 \pm 0.043, n=109$; CAPS-1/2 DKO: $0.191 \pm 0.046$, $\mathrm{n}=115$ / $\mathrm{P}=0.4204$, n.s.; Fig. $3.5 \mathrm{H}$ ), confirming previous findings (Jockusch et al., 2007). 


\subsection{D-ET analysis of synaptic vesicle docking in CAPS-1/2 DKO neurons}

In the electron tomographic three-dimensional analysis, I analyzed samples from three CAPS-1/2 DKO ( $\mathrm{N}=3$ ) and three control animals $(\mathrm{N}=3)$ (Fig. 3.6 A-F). I noticed that CAPS1/2 DKO synaptic profiles analyzed by electron tomography exhibited a drastic reduction in the number of membrane-associated vesicles $(0-2 \mathrm{~nm})$ in comparison to control synaptic profiles (control: $1.202 \pm 0.143, \mathrm{n}=22$; CAPS-1/2 DKO: $0.083 \pm 0.026, \mathrm{n}=19$ / $\mathrm{P}<0.001,{ }^{* * *}$; Fig. $\left.3.6 \mathrm{I}\right)$, and also a strong decrease in the number of closely tethered $(0-4$ $\mathrm{nm}$ ) synaptic vesicles (control: $1.598 \pm 0.158, \mathrm{n}=22$; CAPS-1/2 DKO: $0.180 \pm 0.063, \mathrm{n}=19$ $/ \mathrm{P}<0.001,{ }^{* * *}$; Fig. $\left.3.6 \mathrm{~J}\right)$. However, the number of vesicles within $40 \mathrm{~nm}$ of the presynaptic plasma membrane (control: $2.660 \pm 0.196, n=22$; CAPS-1/2 DKO: $2.393 \pm$ $0.191, \mathrm{n}=19$ / $\mathrm{P}=0.3400$, n.s.; Fig. $3.6 \mathrm{H}$ ) and the number of vesicles within $100 \mathrm{~nm}$ of the presynaptic release sites were unaltered (control: $7.121 \pm 0.413, n=22$; CAPS-1/2 DKO: $6.015 \pm 0.391, \mathrm{n}=19$ / $\mathrm{P}=0.0614$, n.s.; Fig. $3.6 \mathrm{G}$ ). In contrast to synapses lacking Munc13s, synaptic vesicles in synapses lacking both CAPS isoforms appeared to be randomly distributed within the first $100 \mathrm{~nm}$ from the active zone membrane, rather than accumulating at an 8-10 nm distance (Fig. 3.6 M). Moreover, neither the mean outer synaptic vesicle diameter (control: $45.19 \pm 0.560, n=22$; CAPS-1/2 DKO: $45.54 \pm 0.649$, $\mathrm{n}=19$ / $\mathrm{P}=0.6434$, n.s.; Fig. $3.6 \mathrm{~K}, \mathrm{~N}$ ) nor the mean outer synaptic vesicle volume were significantly different from controls (control: $49181 \pm 1896, n=22$; CAPS-1/2 DKO: $50355 \pm$ 2022, $n=19$ / P=0.6743, n.s.; Fig. 3.6 L) for CAPS-1/2 DKO neurons.

In my analysis, $63 \%$ of all sampled CAPS-1/2 DKO synaptic profiles (12 out of 19 tomograms) were completely devoid of membrane-attached vesicles $(0-2 \mathrm{~nm})$ and $58 \%$ of all CAPS-1/2 DKO synaptic profiles (11 out of 19 tomograms) also lacked vesicles closely tethered to the active zone membrane $(0-4 \mathrm{~nm})$. In comparison to control synaptic profiles, the number of membrane-attached $(0-2 \mathrm{~nm})$ and closely tethered $(0-4 \mathrm{~nm})$ synaptic vesicles in CAPS-1/2 DKO neurons was reduced to $7 \%$ and $12 \%$, respectively. Tomograms from CAPS-1/2 DKO synapses that still harbored membrane-attached synaptic vesicles, docking $(0-2 \mathrm{~nm})$ and close tethering $(0-4 \mathrm{~nm})$ was reduced to $19 \%$ and $28 \%$ compared to controls, respectively. 


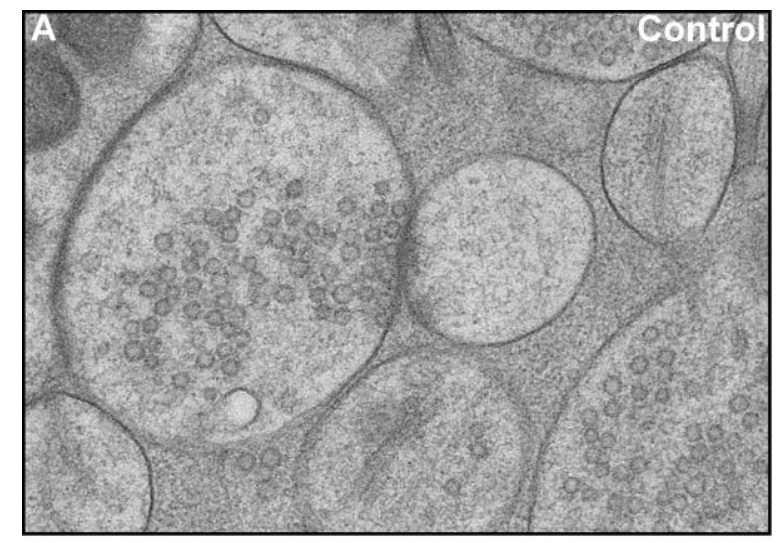

C

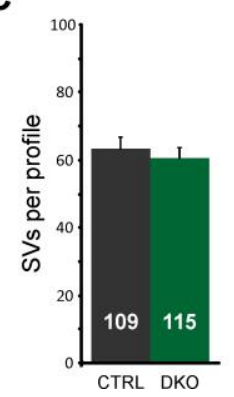

D

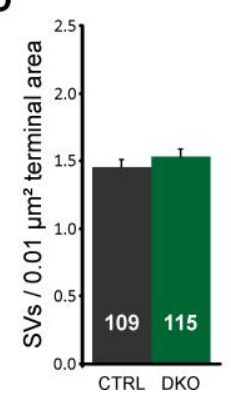

E

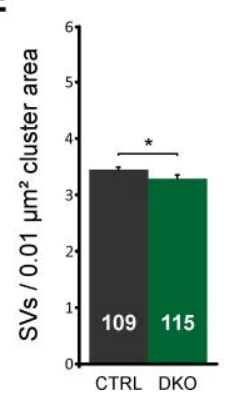

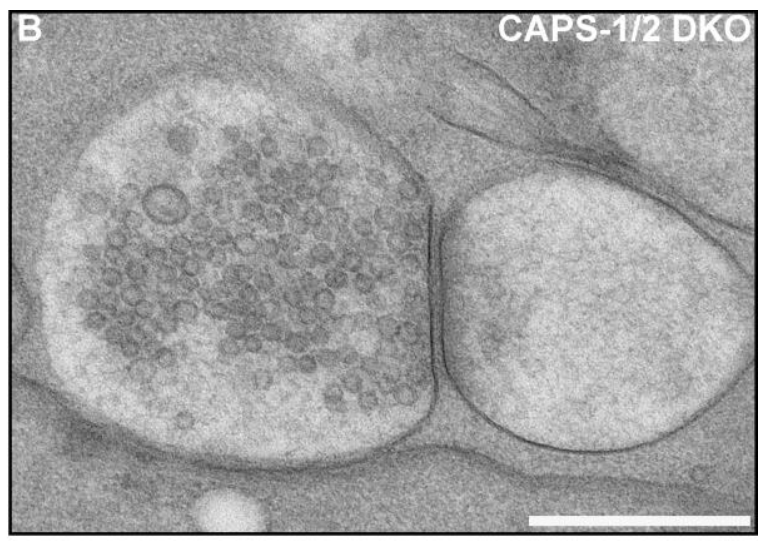

$\mathbf{F}$

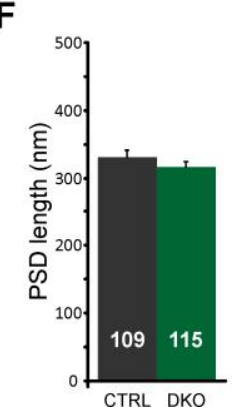

G

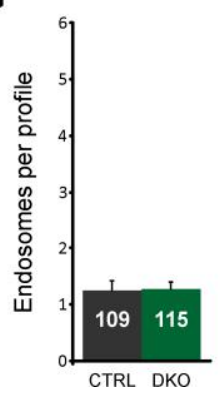

H

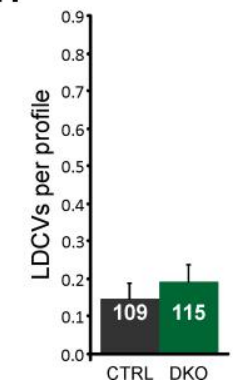

Figure 3.5. Two-dimensional ultrastructural analysis of synaptic morphology in CAPS-1/2 DKO neurons

Electron micrographs of control (A) and CAPS-1/2 DKO (B) synaptic profiles acquired from $60 \mathrm{~nm}$ thick ultrathin sections. Mean number of synaptic vesicles (SVs) per synaptic profile (C). Mean number of SVs normalized to synaptic terminal area (SV terminal density; D). Mean number of SVs normalized to SV cluster area (SV cluster density; E). Mean postsynaptic density (PSD) length (F) Mean number of endosomes per synaptic profile $(\mathbf{G})$. Mean number of large dense-core vesicles (LDCVs) per synaptic profile (H). C-H: Control: $\mathrm{N}=3, \mathrm{n}=109$; CAPS-1/2 DKO: $\mathrm{N}=4, \mathrm{n}=115$ (Mean + SEM), $P<0.001:^{* * *} ; \mathrm{P}<0.01$ : ${ }^{* *} ; \mathrm{P}<0.05:{ }^{*}$. Scale bar: $B, 500 \mathrm{~nm}$.

These ultrastructural findings indicate that CAPS proteins perform not only to produce functionally prime synaptic vesicles, but, similar to Munc13s, have an important role in docking synaptic vesicles to the presynaptic active zone membrane. I interpret these findings as further support for the hypothesis that the RRP of functionally primed vesicles is comprised of membrane-attached synaptic vesicles capable of securing fast synaptic release (Murthy et al., 2001; Schikorski and Stevens, 2001). This pool of readilyreleasable and docked vesicles has been shown here to be either completely depleted or strongly diminished in neurons lacking proteins from the Munc13 or CAPS family. 

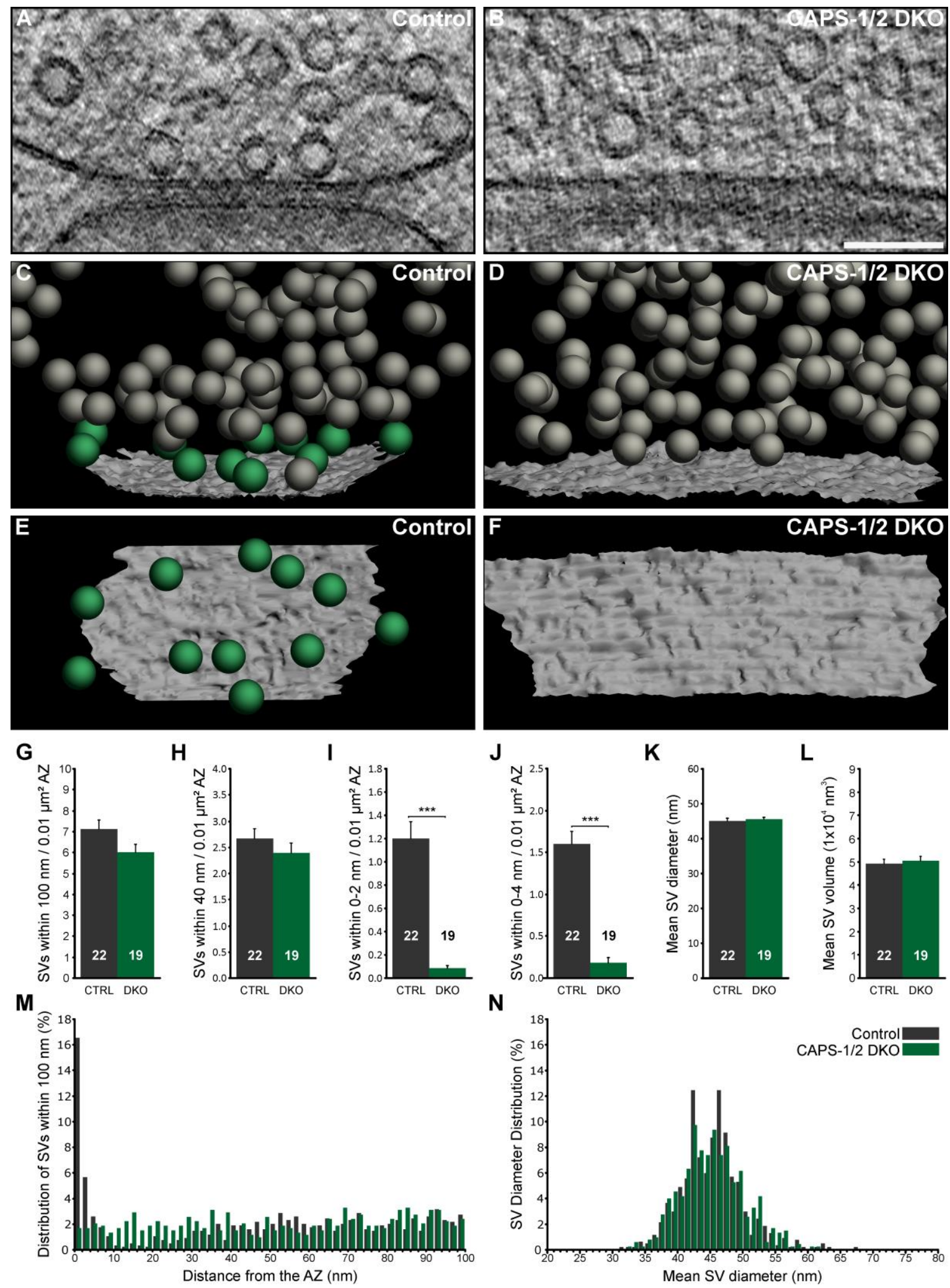

Figure 3.6. Three-dimensional electron tomographic analysis of synaptic vesicle docking in CAPS-1/2 DKO neurons

Tomographically reconstructed subvolumes of control (A) and CAPS-1/2 DKO (B) synapses from $200 \mathrm{~nm}$-thick sections. Three-dimensional models of tomographically reconstructed control (C) and CAPS-1/2 DKO (D) synaptic profiles. Ultrastructural features reconstructed in the models include the active zone plasma membrane (white) and synaptic vesicles (membrane-attached, green; non- 
attached, grey). Orthogonal views of control (E) and CAPS-1/2 DKO (F) tomographic models displaying the spatial arrangement of membrane-attached synaptic vesicles within the reconstructed active zone area. Mean number of SVs within $100 \mathrm{~nm}$ of the $A Z$ normalized to $A Z$ area $(\mathbf{G})$. Mean number of SVs within $40 \mathrm{~nm}$ of the $A Z$ normalized to $A Z$ area $(\mathbf{H})$. Mean number of membrane-attached SVs (within 0-2 nm of the AZ) normalized to AZ area (I). Mean number of membrane-attached and closely-tethered SVs (within 0-4 nm of the AZ) normalized to AZ area (J). Mean outer SV diameter (K). Mean SV volume including the membrane bilayer (L). Spatial distribution of SVs within a $100 \mathrm{~nm}$ distance of the AZ membrane (M). Distribution of synaptic vesicle diameters of vesicles within $100 \mathrm{~nm}$ of the AZ (N). G-L: Control: N=3, n=22; CAPS-1/2 DKO: $\mathrm{N}=3, \mathrm{n}=19$ (Mean + SEM), $\mathrm{P}<0.001:^{* * *} ; \mathrm{P}<0.01:^{* *} ; \mathrm{P}<0.05:^{*} . \mathbf{M}, \mathbf{N}:(716 \mathrm{SVs}$ in control and 560 SVs in CAPS-1/2 DKO synaptic profiles). Scale bar: B,100 nm.

\subsubsection{The role of the t-SNARE SNAP25 in synaptic vesicle docking}

Since Munc13s are believed to open Syntaxin-1 to enable t-SNARE acceptor complex formation with SNAP25, and CAPS proteins have been shown to facilitate SNARE complex formation in vitro, I decided to reinvestigate the role of SNARE proteins SNAP25, Syntaxin-1 and Synaptobrevin-2 in synaptic vesicle docking in my experimental setting (Daily et al., 2010; James et al., 2009, 2010; Ma et al., 2011). In order to understand the function of SNARE proteins in the synaptic vesicle membrane-attachment process, I first decided to analyze mouse mutants that show no or decreased expression of the neuronal t-SNAREs SNAP25 and Syntaxin-1. Both t-SNARE molecules have been implicated in secretory vesicle docking in non-neuronal systems (de Wit et al., 2006, 2009; Wu et al., 2012).

SNAP25 mice (Washbourne et al., 2002) were received from Maarten Loos (Amsterdam) and Ina Herfort (Göttingen). Neurons from SNAP25 mutant mice were described to slowly degenerate in dissociated neurons cultures beyond DIV 7. However, previous studies showed that high-density cultures can prolong neuronal survival of SNAP25 KO neurons (Bronk et al., 2007; Washbourne et al., 2002). In my hippocampal organotypic slice culture system, slices from SNAP25 KO animals were considerably thinner than control slices, however, immunhistochemistry revealed an increased density and a larger mean area of VGLUT1-immunoreactive puncta (See section 3.1.3.3.3). Previous physiological analysis of autaptic neurons lacking SNAP25 revealed an $88 \%$ reduction in the RRP size in KO neurons in comparison to control neurons (Bronk et al., 2007). 


\subsection{D-EM analysis of synaptic morphology in SNAP25 KO neurons}

As wild-type and mice heterozygous for SNAP25 were phenotypically indistinguishable at birth, SNAP25 heterozygous mice were used as controls for this study (Delgado-Martínez et al., 2007). For the analysis of two-dimensional electron micrographs from synaptic profiles of ultrathin sections, organotypic slices from six SNAP25 KO mice $(\mathrm{N}=6)$ were compared with slices from six littermate mice $(\mathrm{N}=6)$ heterozygous for the SNAP25 allele in three cultures. The gross synaptic morphology was unaltered between control and SNAP25 KO samples, but similar to Munc13-1/2 DKO synapses, the presence of larger vesicle sizes in SNAP25 KO synapses was apparent (Fig. 3.7 A, B). The total number of synaptic vesicles per synaptic profile did not significantly differ between $\mathrm{KO}$ and control cultures (control: $68.73 \pm 3.790, \mathrm{n}=82$; SNAP25 KO: $63.74 \pm 3.276, \mathrm{n}=101 / \mathrm{P}=0.4061$, n.s.; Fig. $3.7 \mathrm{C}$ ), and no change in the PSD length was observed (control: $374.1 \pm 15.62$, $\mathrm{n}=82$; SNAP25 KO: $351.6 \pm 11.31, \mathrm{n}=101$ / $\mathrm{P}=0.3844$, n.s.; Fig. $3.7 \mathrm{~F})$. However, both the synaptic vesicle terminal density (control: $1.688 \pm 0.074, \mathrm{n}=82$; SNAP25 KO: $1.446 \pm$ 0.051, $n=101 / \mathrm{P}=0.0245$, *; Fig. $3.7 \mathrm{D})$ and the synaptic vesicle cluster density (control: $4.712 \pm 0.084, \mathrm{n}=82$; SNAP25 KO: $3.785 \pm 0.053, \mathrm{n}=101 / \mathrm{P}<0.001,{ }^{* * *}$; Fig $\left.3.7 \mathrm{E}\right)$ were significantly reduced. Moreover, a dramatic increase in the number of large, vesicular, possibly endosomal structures, in SNAP-25 KO synaptic profiles was detected (control: $1.415 \pm 0.247, \mathrm{n}=82$; SNAP25 KO: $4.168 \pm 0.456, \mathrm{n}=101 / \mathrm{P}<0.001$, ${ }^{* \star}$; Fig. $3.7 \mathrm{G}$ ). This finding supports the hypothesis that SNAP25 may play additional roles in vesicle recycling or trafficking pathway (Aikawa et al., 2006; Peng et al., 2013). In addition, I observed a slight, though statistically insignificant increase in the number of LDCVs per synaptic profile in synapses lacking SNAP25 (control: $0.183 \pm 0.046, n=82$; SNAP25 KO: $0.317 \pm$ 0.064, n=101 / P=0.2601, n.s.; Fig. 3.7 H).

\subsection{D-ET analysis of synaptic vesicle docking in SNAP25 KO neurons}

For the electron tomographic analysis, organotypic slices from five SNAP25 KO mice $(\mathrm{N}=5)$ were compared with slices from five littermate mice $(\mathrm{N}=5)$ heterozygous for the SNAP25 allele in three cultures (Fig. 3.8 A-F). Tomographic analysis revealed a strong reduction in membrane-attached synaptic vesicles $(0-2 \mathrm{~nm})$ in SNAP25 KO synaptic profiles compared to controls (control: $1.125 \pm 0.130$, $n=24$; SNAP25 KO: $0.241 \pm 0.062$, $\mathrm{n}=25 / \mathrm{P}<0.001,{ }^{* * *}$; Fig. $\left.3.8 \mathrm{I}\right)$ as well as synaptic vesicles closely tethered $(0-4 \mathrm{~nm})$ to the presynaptic release site (control: $1.462 \pm 0.179, n=24$; SNAP25 KO: $0.508 \pm 0.101, n=25$ / 
$\mathrm{P}<0.001,{ }^{* *}$; Fig. $\left.3.8 \mathrm{~J}\right)$. In contrast to this strong reduction in the number of membraneassociated synaptic vesicles, the number of membrane proximal synaptic vesicles (0-40 $\mathrm{nm}$ ) was unaltered (control: $2.779 \pm 0.219, \mathrm{n}=24$; SNAP25 KO: $2.749 \pm 0.132, \mathrm{n}=25$ / $\mathrm{P}=0.9047$, n.s.; Fig. $3.8 \mathrm{H}$ ).

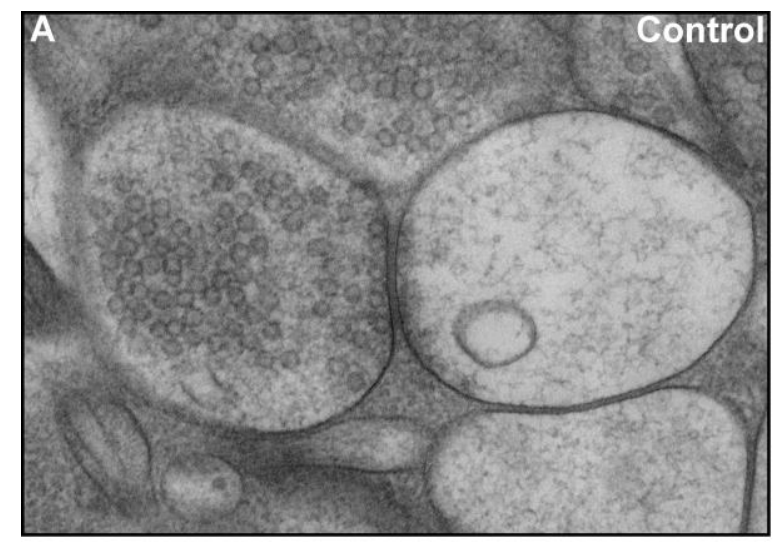

C

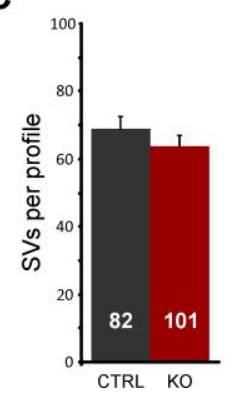

D

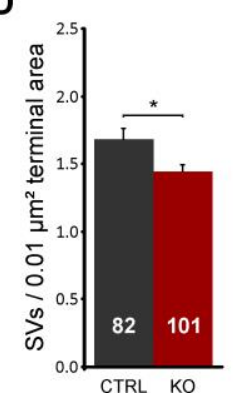

E

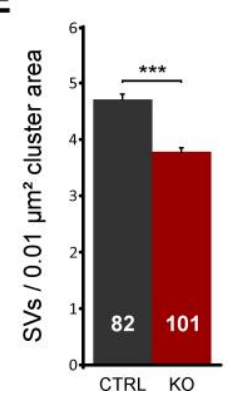

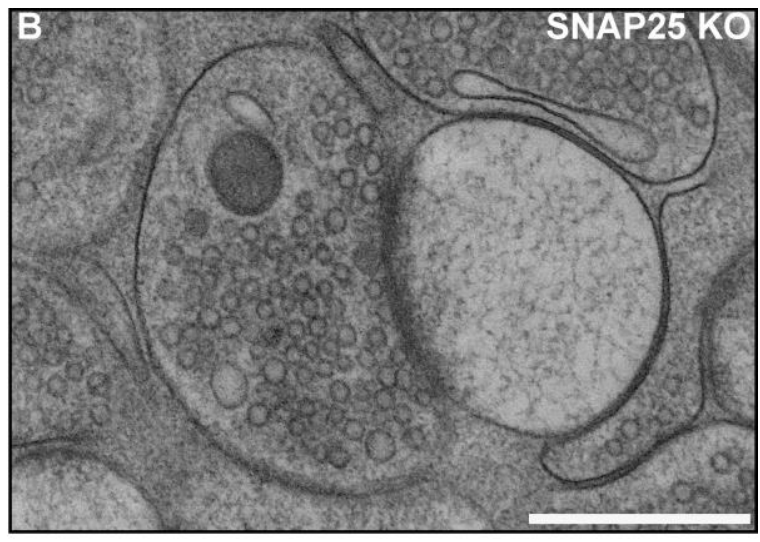

$\mathbf{F}$

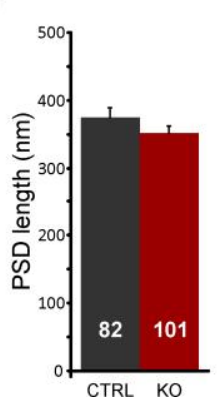

G

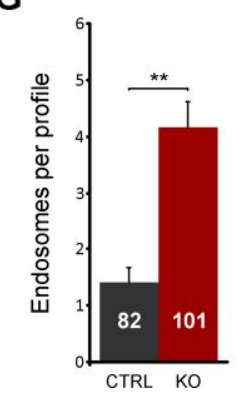

H

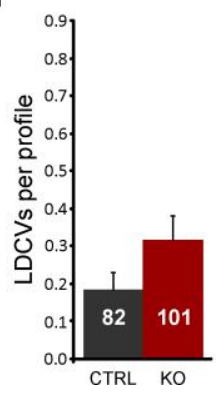

Figure 3.7. Two-dimensional ultrastructural analysis of synaptic morphology in SNAP25 KO neurons

Electron micrographs of control (A) and SNAP25 KO (B) synaptic profiles acquired from $60 \mathrm{~nm}$ thick ultrathin sections. Mean number of synaptic vesicles (SVs) per synaptic profile (C). Mean number of SVs normalized to synaptic terminal area (SV terminal density; D). Mean number of SVs normalized to SV cluster area (SV cluster density; E). Mean postsynaptic density (PSD) length (F) Mean number of endosomes per synaptic profile (G). Mean number of large dense-core vesicles (LDCVs) per synaptic profile (H). C-H: Control: $\mathrm{N}=6, \mathrm{n}=82$; SNAP25 KO: $\mathrm{N}=6, \mathrm{n}=101$ (Mean + SEM), $\mathrm{P}<0.001$ : $^{* * *} ; \mathrm{P}<0.01:^{* *} ; \mathrm{P}<0.05:^{*}$. Scale bar: $\mathrm{B}, 500 \mathrm{~nm}$.

The number of vesicles within $100 \mathrm{~nm}$ distance from the active zone was only slightly reduced (control: $6.766 \pm 0.396, n=24$; SNAP25 KO: $5.682 \pm 0.236, n=25$ / $P=0.0216$, *; Fig. $3.8 \mathrm{G})$. In this analysis, 13 out of 25 tomograms from SNAP25 KO synapses (52\%) were completely devoid of membrane-attached vesicles $(0-2 \mathrm{~nm})$, with 7 out of 25 tomograms (28\%) lacking both physically docked and closely tethered synaptic vesicles $(0-4 \mathrm{~nm})$. In the absence of SNAP25, the numbers of membrane-attached and closely tethered vesicles were reduced to $21 \%$ and $35 \%$ of controls, respectively. Moreover, in tomograms from SNAP25 KO synapses that still harbored membrane-attached synaptic vesicles, docking $(0-2 \mathrm{~nm})$ and close tethering $(0-4 \mathrm{~nm})$ was reduced to $41 \%$ and $64 \%$ of controls, respectively. 

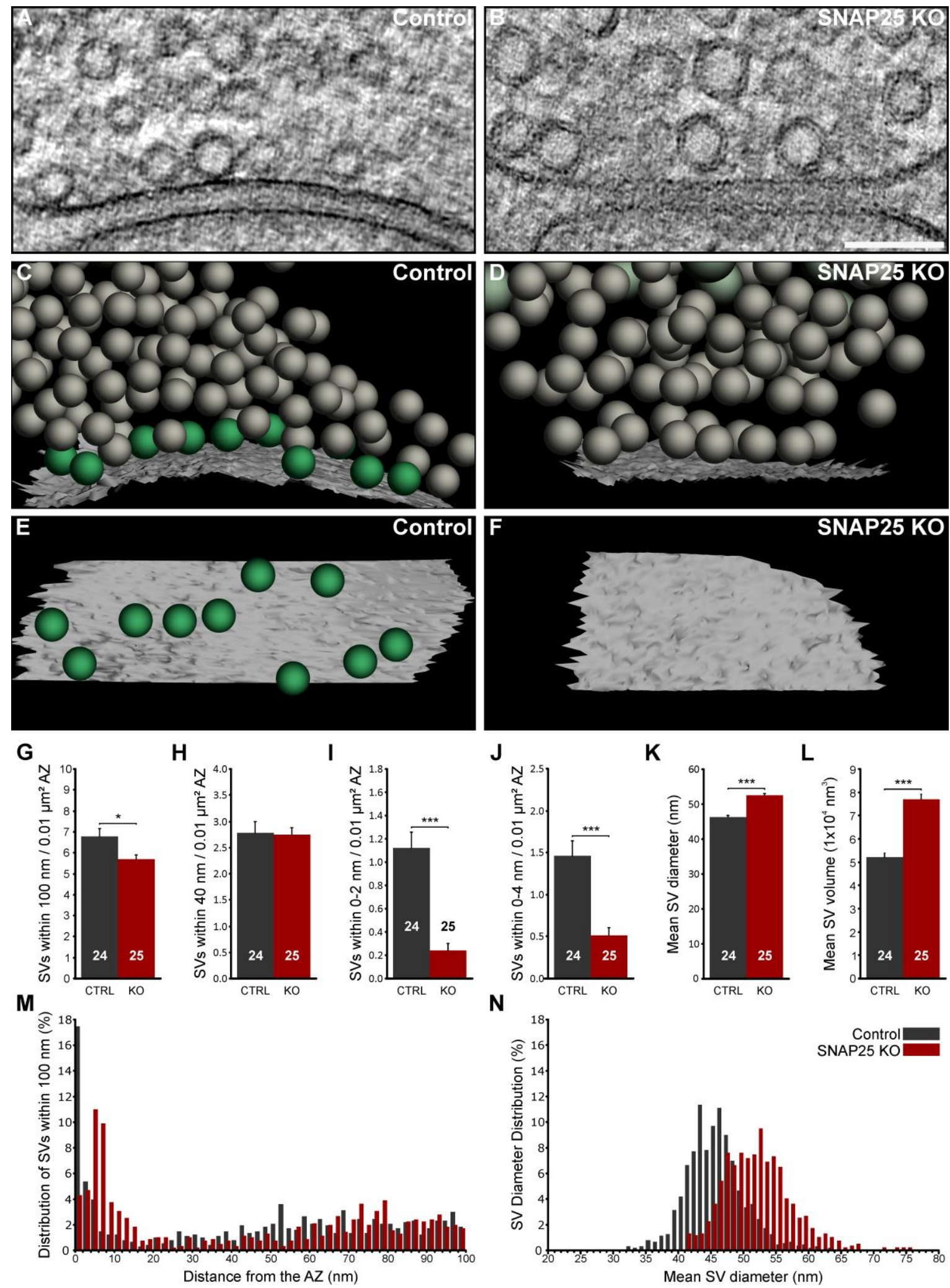

Figure 3.8. Three-dimensional electron tomographic analysis of synaptic vesicle docking in SNAP25 KO neurons

Tomographically reconstructed subvolumes of control (A) and SNAP25 KO (B) synapses from 200 nm-thick sections. Three-dimensional models of tomographically reconstructed control (C) and SNAP25 KO (D) synaptic profiles. Ultrastructural features reconstructed in the models include the active zone plasma membrane (white), synaptic vesicles (membrane-attached, green; nonattached, grey) and endosomes (light blue). Orthogonal views of control (E) and SNAP25 KO (F) 
tomographic models displaying the spatial arrangement of membrane-attached synaptic vesicles within the reconstructed active zone area. Mean number of SVs within $100 \mathrm{~nm}$ of the AZ normalized to $A Z$ area (G). Mean number of SVs within $40 \mathrm{~nm}$ of the $A Z$ normalized to $A Z$ area $(\mathbf{H})$. Mean number of membrane-attached SVs (within 0-2 nm of the AZ) normalized to AZ area (I). Mean number of membrane-attached and closely-tethered SVs (within 0-4 nm of the AZ) normalized to $A Z$ area $(\mathbf{J})$. Mean outer SV diameter (K). Mean SV volume including the membrane bilayer (L). Spatial distribution of SVs within a $100 \mathrm{~nm}$ distance of the AZ membrane (M). Distribution of synaptic vesicle diameters of vesicles within $100 \mathrm{~nm}$ of the AZ (N). G-L: Control: $\mathrm{N}=5, \mathrm{n}=24$; SNAP25 KO: $\mathrm{N}=5, \mathrm{n}=25$ (Mean + SEM), $\mathrm{P}<0.001$ : $^{* * *} ; \mathrm{P}<0.01:{ }^{* *} ; \mathrm{P}<0.05:{ }^{*} . \mathbf{M}, \mathbf{N}:(850$ SVs in control and 732 SVs in SNAP25 KO synaptic profiles).Scale bar: B,100 nm.

Similar to synapses lacking Munc13s, terminals that were devoid of the neuronal t-SNARE SNAP25 showed a prominent accumulation of synaptic vesicles at a distance of 4-8 nm from the active zone membrane (Fig. $3.8 \mathrm{M}$ ). These membrane-proximal, but not physically docked, vesicles were often linked to the presynaptic plasma membrane by thin, long filaments, but were not connected to the membrane by multiple short tethers at this distance. Moreover, I observed an enormous increase in the mean outer vesicle diameter in SNAP25 KO synapses (control: $46.12 \pm 0.482, \mathrm{n}=24$; SNAP25 KO: $52.53 \pm$ $0.486, n=25 / P<0.001$, ${ }^{* * *}$; Fig. $\left.3.8 \mathrm{~K}, \mathrm{~N}\right)$, resulting in $48 \%$ increase in the mean vesicular volume (control: $52132 \pm 1682, n=24$; SNAP25 KO: $76926 \pm 2191, n=25 / P<0.001$, ***; Fig. 3.8 L), supporting the idea that SNAP25 might have an additional role in presynaptic membrane recycling (Peng et al. 2013; Xu et al. 2013).

In our heterozygous control animals from two different cultures we observed two synapses that do not harbor many docked vesicles, but show small, omega-shaped membrane invaginations, active zone regions with concave curvature described as full-collapse fusion events (Fernández-Busnadiego et al., 2010), and large endocytotic structures connected to the plasma membrane in the perimeter of the active zone (data not shown). These structures are most likely features of synapses that had been captured in or shortly after the process of neurotransmitter release. I occasionally observe these structures in other genotypes, albeit with a significantly lower frequency. Since control synapses lacking these ultrastructural correlates of high synaptic activity displayed normal numbers of docked vesicles, we decided to continue our analysis with the heterozygous control group. A previous analysis of the neurological Coloboma mouse $(\mathrm{Cm} /+)$, which lacks one copy of the SNAP25 gene, revealed an epileptic phenotype caused by synaptic hyperexcitability due to altered voltage-gated calcium channel modulation (Corradini et al., 2012; Zhang et al., 2004). Mice heterozygous for SNAP25 may therefore exhibit increased slice activity leading to the detection or more dynamic fusion and endocytotic events. 


\subsection{SNAP23 does not compensate for the loss of SNAP25}

In the present study, organotypic hippocampal slices from SNAP25 KO mice underwent considerable thinning during the first weeks in culture, consistent with the reported degeneration of SNAP25 KO neurons in low density culture (Bronk et al., 2007; Washbourne et al., 2002). To assess the impact of this on local excitatory networks, we decided to evaluate the spatial density of glutamatergic synapses in our preparation. Moreover, we were interested to see whether SNAP23, another well-characterized SNAP isoform could potentially compensate for the loss of SNAP25 in the surviving neurons. Overexpression of SNAP23 in SNAP25 KO neurons has been shown to rescue the reduction in RRP sizes, but not the almost complete loss of evoked responses (DelgadoMartínez et al., 2007)

Immunohistochemistry studies and the imaging analysis were performed by Dr. Benjamin Cooper, Department of Molecular Neurobiology of the Max-Planck Institute of Experimental Medicine, Göttingen, Germany. Organotypic slices from two wild-type and two SNAP25 KO animals were fixed after 4-5 weeks in culture and processed for immunohistochemistry by using antibodies against SNAP23, SNAP25 and VGLUT1. VGLUT1, the vesicular glutamate transporter, resides in the synaptic vesicle membrane in glutamatergic synapses and therefore serves as an appropriate marker for excitatory spine synapses, the synaptic subtype used for ultrastructural analyses in this study. SNAP25 immunoreactivity in wild-type slices was localized along the neuronal processes and at synapses, but was absent in SNAP25 KO slices (data not shown). Punctate SNAP23 labeling was distributed throughout the slices in both, wild-type and KO conditions (Fig. S1 A, D). Although absent from excitatory presynapses, SNAP23 was often observed in close apposition to VGLUT1 signals, indicating a potential postsynaptic localization of the protein in wild-type and SNAP25 KO synapses (Fig. S1 B, C, E ,F). The density of SNAP23 signals in SNAP25 KO slices normalized to control samples (control: $1.000 \pm 0.060, \mathrm{n}=18$; SNAP25 KO: $1.363 \pm 0.105, \mathrm{n}=18 / \mathrm{P}=0.005,{ }^{* *}$; Fig. S1 G) and the density of VGLUT1 positive puncta in SNAP25 KO samples normalized to control cultures is increased (control: $1 \pm 0.055, \mathrm{n}=18$; SNAP25 KO: $1.718 \pm 0.045, \mathrm{n}=18 / \mathrm{P}<0.001$, ***; Fig. $\mathrm{S} 1 \mathrm{H}$ ). Moreover, a significant increase in the sizes of VGLUT1 puncta was detected in SNAP25 KO slices (control: $1 \pm 0.036, \mathrm{n}=18$; SNAP25 KO: $1.792 \pm 0.056, \mathrm{n}=18$ / $\mathrm{P}<0.001,{ }^{* * *}$; Fig. S1 I).

In summary, mature SNAP25 KO organotypic hippocampal slices exhibit a high density of excitatory synapses despite the cell loss in the first week of culturing. Moreover, the 
number of VGLUT1 positive puncta increases in SNAP25 KO slices likely indicating a compensatory effect. The increase of the size of VGLUT1-positive puncta could reflect either the increased synaptic vesicle sizes observed in electron tomograms or retention of VGLUT1 in the presynaptic membrane as a consequence of disrupted endocytosis. Since the methods used to quantify the number and area of puncta are sensitive to signal intensities, it is possible that a compensatory increase in VGLUT1 expression in SNAP25 deficient neurons brings a greater number of VGLUT1 synapses above the intensity threshold for inclusion in the analysis, thereby explaining the observed increase in VGLUT1 density. Considering the lack of colocalization with VGLUT, it is however unlikely that SNAP23 can compensate for the loss of SNAP25 in surviving neurons.

\subsubsection{The role of the t-SNARE Syntaxin-1 in synaptic vesicle docking}

Since Munc13s are thought to regulate SNARE complex formation by open Syntaxin-1 to trigger SNARE nucleation, we were interested in the role of the second t-SNARE Syntaxin-1 in synaptic vesicle docking. First, we wanted to analyze a double mutant mouse line deficient for Syntaxin-1A and $-B$, which recently became available in the laboratory of Prof. Christian Rosenmund, Berlin. Unfortunately, these mice displayed a strong neurodegenerative phenotype, comparably severe to that described for Munc18-1 $\mathrm{KO}$ neurons in culture (Verhage et al., 2000). As I failed to rescue or significantly prolong survival of the slices by employing the organotypic slice culture system, we selected a Syntaxin-1B Knock-in mouse line, which expresses Syntaxin-1B fused to YFP in the Syntaxin-1A KO background (Syntaxin-1A KO/ -B ${ }^{\mathrm{YFP}}$ ) for further analyses (Arancillo et al., 2013; Gerber et al., 2008). This mouse line permits the study of neurons with reduced Syntaxin-1 levels under hypomorphic conditions, but prevents the cultured cell death characteristic of the full KO. No significant alterations in basic physiological characteristics were detected in Syntaxin-1 $\mathrm{A}^{-/}$, Syntaxin- $1 \mathrm{~B}^{+/+}$and Syntaxin- $1 \mathrm{~A}^{-/-}$, Syntaxin-1B $\mathrm{B}^{\mathrm{yf} /+}$ autapic neuron cultures, indicating that one functional allele of Syntaxin-1B is enough for normal synaptic function (Arancillo et al., 2013). For this study, I therefore pooled control data from two Syntaxin-1 $A^{-/}$, Syntaxin- $1 B^{+/+}$and four Syntaxin- $1 A^{-/-}$, Syntaxin-1 $B^{y f p /+}$ mice and compared them with data from six Syntaxin-1A KO/ $-B^{\text {YFP }}$ mice from two independent cultures. 

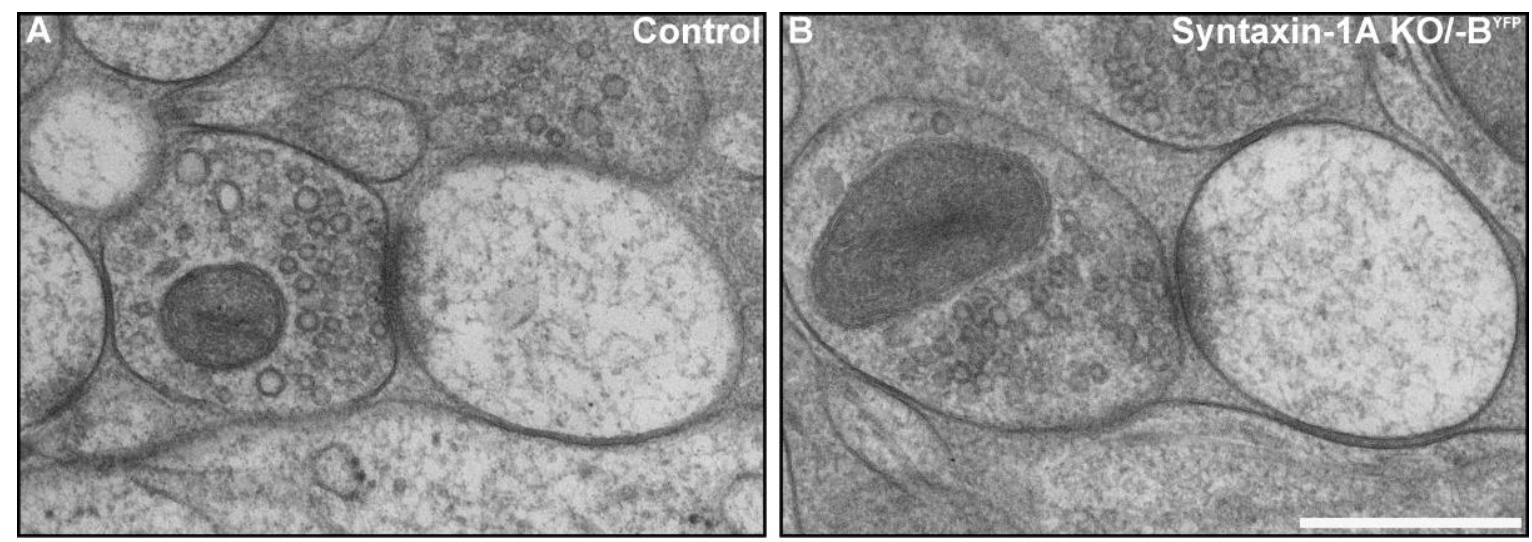

C

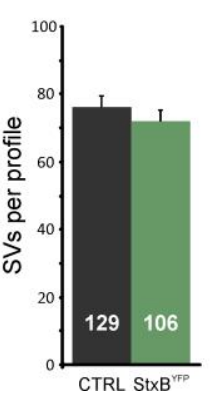

D

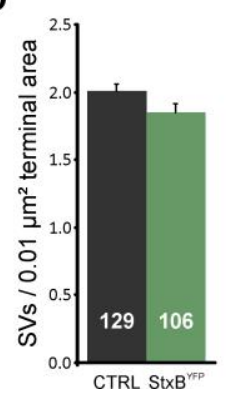

E

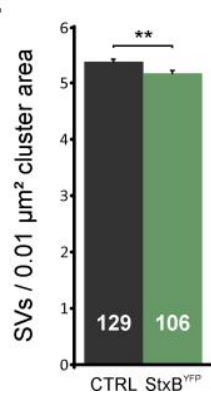

$\mathbf{F}$

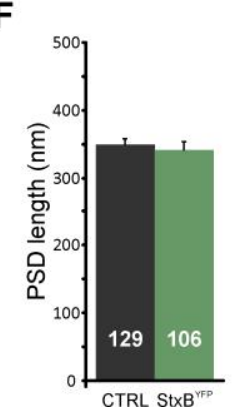

G

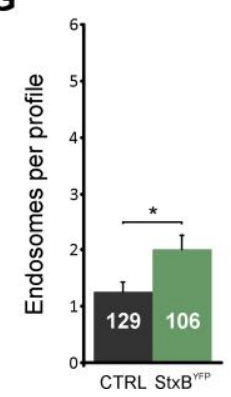

H

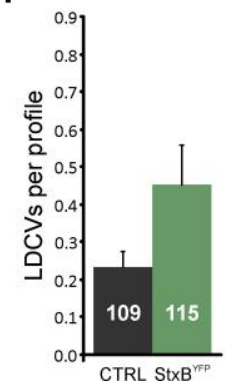

Figure 3.9. Two-dimensional ultrastructural analysis of synaptic morphology in Syntaxin-1A $\mathrm{KO} / \mathrm{B}^{\mathrm{YFP}}$ neurons

Electron micrographs of control $(\mathbf{A})$ and Syntaxin-1A KO/ $-B^{\text {YFP }}$ (B) synaptic profiles acquired from $60 \mathrm{~nm}$-thick ultrathin sections. Mean number of synaptic vesicles (SVs) per synaptic profile (C). Mean number of SVs normalized to synaptic terminal area (SV terminal density; D). Mean number of SVs normalized to SV cluster area (SV cluster density; E). Mean postsynaptic density (PSD) length $(\mathbf{F})$ Mean number of endosomes per synaptic profile $(\mathbf{G})$. Mean number of large dense-core vesicles (LDCVs) per synaptic profile (H). C-H: Control: $N=6, n=129$; Syntaxin-1A KO/ $-B^{Y F P}: \mathrm{N}=6$, $\mathrm{n}=106$ (Mean + SEM), $\mathrm{P}<0.001$ : $^{* \star *} ; \mathrm{P}<0.01:^{* *} ; \mathrm{P}<0.05:{ }^{*}$. Scale bar: B, $500 \mathrm{~nm}$.

\subsection{D-EM analysis of synaptic morphology in Syntaxin-1A $\mathrm{KO} /-\mathrm{B}^{\mathrm{YFP}}$ neurons}

Reduction of Syntaxin-1 levels in the Syntaxin-1A KO/ $-B^{\text {YFP }}$ mouse line did not affect gross synaptic morphology, which was comparable to that of controls in electron micrographs from ultrathin sections (Fig. 3.9 A, B). The number of synaptic vesicles per synaptic profile was unchanged between Syntaxin-1A KO/ $-\mathrm{B}^{\mathrm{YFP}}$ and control samples (control: $76.25 \pm 3.179, n=129$; Syntaxin-1A KO/ -B ${ }^{\text {YFP }}: 71.79 \pm 3.575, n=106 / P=0.2609$, n.s.; Fig. $3.9 \mathrm{C}$ ). No significant changes were observed in either the PSD length (control: $349.8 \pm 8.515, n=129$; Syntaxin-1A KO/ $-B^{Y F P}: 342.0 \pm 11.65, n=106 / P=0.2569$, n.s.; Fig. $3.9 \mathrm{~F}$ ) or the synaptic vesicle terminal density (control: $2.006 \pm 0.058, n=129$; Syntaxin-1A $\mathrm{KO} /-\mathrm{B}^{\mathrm{YFP}}: 1.852 \pm 0.065, \mathrm{n}=106 / \mathrm{P}=0.1072$, n.s.; Fig. $\left.3.9 \mathrm{D}\right)$ between the two groups. However, I detected a significant decrease in the synaptic vesicle cluster density (control: 
$5.378 \pm 0.056, n=129 ;$ Syntaxin-1A KO/ $-B^{\text {YFP. }} 5.168 \pm 0.055, n=106 / P=0.0070$, **; Fig. 3.9 E). Similar to Munc13-1/2 DKO and SNAP25 KO synapses, synapses with reduced levels of Syntaxin-1 showed a tendency towards an increased number of endosomal structures per presynaptic profile (control: $1.264 \pm 0.160, n=129$; Syntaxin-1A KO/ $-B^{\text {YFP }}$ : $2.009 \pm 0.253, \mathrm{n}=106$ / P=0.0315, *; Fig. $3.9 \mathrm{G}$ ). In addition, I detected a slight, though not statistically significant, increase in the number of LDCVs per synaptic profile in synapses expressing reduced levels of Syntaxin-1 (control: $0.233 \pm 0.042, n=129$; Syntaxin-1A KO/ $B^{\text {YFP }}: 0.453 \pm 0.106, n=106 / P=0.1893$, n.s.; Fig. 3.9 H).

\subsection{D-ET analysis of synaptic vesicle docking in Syntaxin-1A KO/ -B ${ }^{\text {YFP }}$ neurons}

By electron tomographic analysis, it was first tested whether differences in the number of docked vesicles were detectable between Syntaxin- $1 \mathrm{~A}^{-/}$, Syntaxin- $1 \mathrm{~B}^{+/+}$and Syntaxin- $1 \mathrm{~A}^{-/-}$ , Syntaxin-1 $\mathrm{B}^{\mathrm{yfp} /+}$ mice (graphs not shown). Confirming the physiological findings, I was not able to detect significant changes in the number of active zone membrane-attached vesicles $(0-2 \mathrm{~nm})$ in Syntaxin-1 $\mathrm{A}^{-/}$, Syntaxin-1 $\mathrm{B}^{+/+}$synaptic profiles in comparison to Syntaxin-1 $A^{-/}$, Syntaxin-1 $B^{y f p /+}$ synaptic profiles (Syntaxin-1 $A^{-/}$, Syntaxin-1B ${ }^{+/+}: 1.028 \pm$ $0.224, N=2, n=6$; Syntaxin-1 $A^{-/-}$, Syntaxin-1 $B^{y p /+}: 1.003 \pm 0.182, N=3, n=15 / P=0.9381$, n.s.). I obtained a similar result, when including those synaptic vesicles that were closely tethered to the presynaptic release site $\left(0-4 \mathrm{~nm}\right.$; Syntaxin-1 $\mathrm{A}^{-/}$, Syntaxin-1B $\mathrm{B}^{+/+}: 1.703 \pm$ $0.319, n=6$; Syntaxin-1 $A^{-/-}$, Syntaxin-1 $\mathrm{B}^{\mathrm{yfp} /+}: 1.338 \pm 0.215, \mathrm{n}=15 / \mathrm{P}=0.3667$, n.s.). I therefore decided to pool the data obtained from both genotypes into one control group. Electron tomographic analysis was performed on organoypic slices from four Syntaxin-1A $\mathrm{KO} /-\mathrm{B}^{\mathrm{YFP}}$ mice $(\mathrm{N}=4)$ and compared with slices from five control mice $(\mathrm{N}=5)$ (Fig. $3.10 \mathrm{~A}$ $\mathrm{F})$. Syntaxin-1A KO/ $-\mathrm{B}^{\mathrm{YFP}}$ presynaptic terminals exhibited a reduction in the number of membrane-attached (0-2 nm; control: $1.010 \pm 0.142, n=21$; Syntaxin-1A KO/ $-B^{\text {YFP }}: 0.623$ $\pm 0.110, \mathrm{n}=23 / \mathrm{P}=0.0356,{ }^{*}$; Fig. $\left.3.10 \mathrm{I}\right)$ and closely tethered vesicles (0-4 nm; control: $1.442 \pm 0.178, n=21$; Syntaxin-1A KO/ $-B^{\text {YFP }}: 0.937 \pm 0.129, n=21 / P=0.0249$, *; Fig. 3.10 J). 

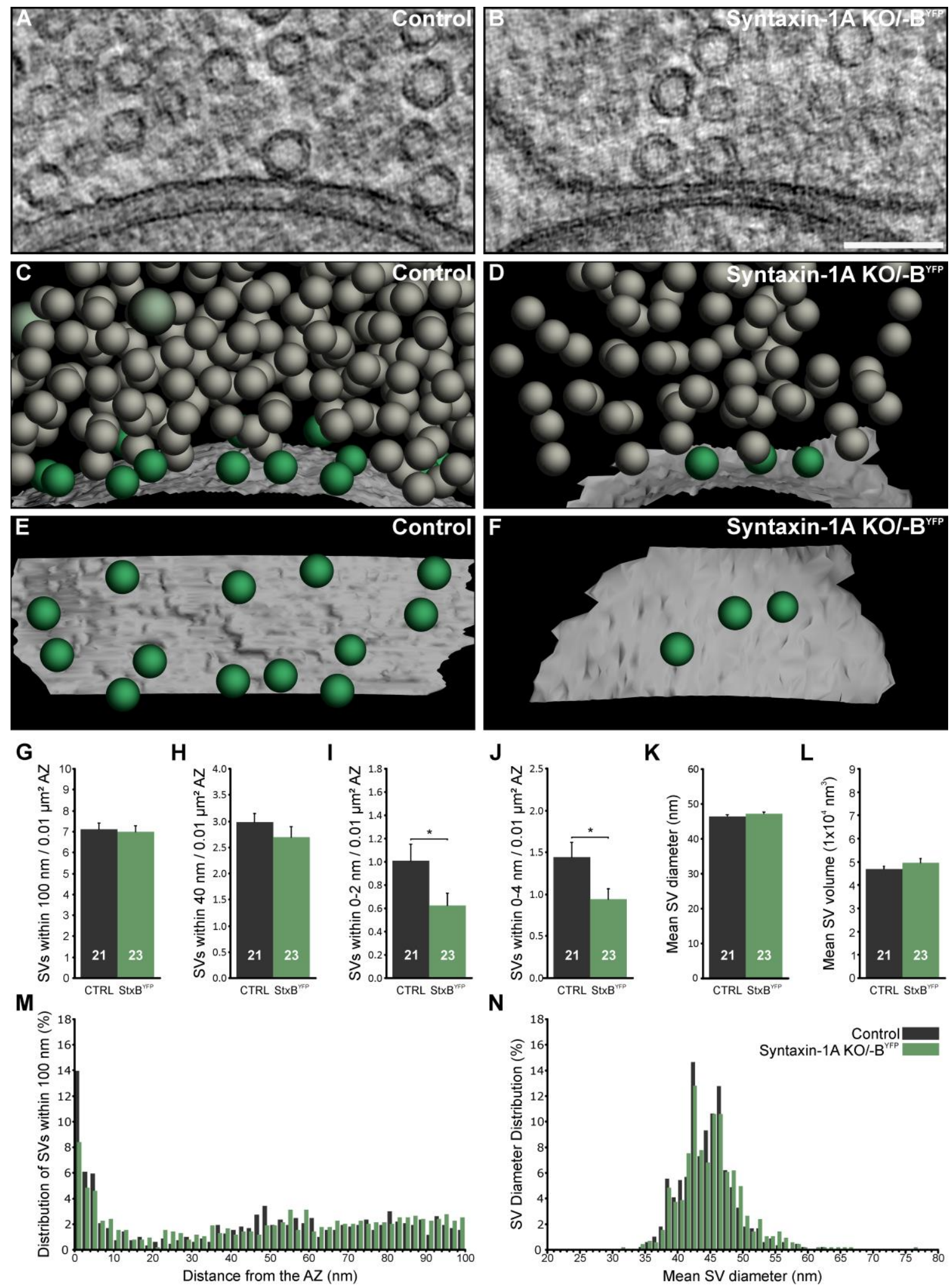

Figure 3.10. Three-dimensional electron tomographic analysis of synaptic vesicle docking in Syntaxin-1A KO/ - ${ }^{\mathrm{YFP}}$ neurons

Tomographically reconstructed subvolumes of control $(\mathbf{A})$ and Syntaxin-1A KO/ -B $\mathrm{YFP}$ (B) synapses from $200 \mathrm{~nm}$-thick sections. Three-dimensional models of tomographically reconstructed control (C) and Syntaxin-1A KO/ -B ${ }^{\text {YFP }}$ (D) synaptic profiles. Ultrastructural features reconstructed in the models include the active zone plasma membrane (white), synaptic vesicles (membrane-attached, 
green; non-attached, grey) and endosomes (light blue). Orthogonal views of control (E) and Syntaxin-1A KO/ $-B^{\text {YFP }}(\mathbf{F})$ tomographic models displaying the spatial arrangement of membraneattached synaptic vesicles within the reconstructed active zone area. Mean number of SVs within $100 \mathrm{~nm}$ of the $A Z$ normalized to $A Z$ area $(\mathbf{G})$. Mean number of SVs within $40 \mathrm{~nm}$ of the $A Z$ normalized to $A Z$ area $(\mathbf{H})$. Mean number of membrane-attached SVs (within 0-2 $\mathrm{nm}$ of the $A Z$ ) normalized to $A Z$ area (I). Mean number of membrane-attached and closely-tethered SVs (within 0$4 \mathrm{~nm}$ of the $\mathrm{AZ}$ ) normalized to $\mathrm{AZ}$ area $(\mathbf{J})$. Mean outer SV diameter $(\mathbf{K})$. Mean SV volume including the membrane bilayer (L). Spatial distribution of SVs within a $100 \mathrm{~nm}$ distance of the $A Z$ membrane (M). Distribution of synaptic vesicle diameters of vesicles within $100 \mathrm{~nm}$ of the AZ $(\mathbf{N})$. G-L: Control: $N=5, n=21$; Syntaxin-1A KO/ $-B^{Y F P}$ : $N=4, n=23$ (Mean + SEM), $P<0.001$ : ***; $P<0.01$ : ${ }^{* *} ; \mathrm{P}<0.05$ : *. M, N: (748 SVs in control and $818 \mathrm{SVs}$ in Syntaxin-1A KO/ $-B^{\text {YFP }}$ synaptic profiles). Scale bar: B, $100 \mathrm{~nm}$.

The number of membrane proximal synaptic vesicles $(0-40 \mathrm{~nm}$; control: $2.978 \pm 0.1712$, $n=21$; Syntaxin-1A KO/ $-B^{\text {YFP. }} 2.693 \pm 0.197, n=23 / P=0.284$, n.s.; Fig. $3.10 \mathrm{H}$ ) and the number of vesicles within $100 \mathrm{~nm}$ distance from the active zone (control: $7.107 \pm 0.312$, $n=21$; Syntaxin-1A KO/ $-B^{\text {YFP. }} 6.996 \pm 0.273, n=23 / P=0.7896$, n.s.; Fig. $3.10 \mathrm{G}$ ) were unaltered. In comparison to control samples, Syntaxin-1A KO/ $-B^{\text {YFP }}$ neurons showed a reduction in the number of membrane-attached and closely tethered vesicles to $62 \%$ and $65 \%$, respectively. Looking at the distribution of synaptic vesicles within $100 \mathrm{~nm}$ of the active zone, one can recognize the reduction in the number of membrane-attached vesicles in the first 0-2 $\mathrm{nm}$ bin, however the general distribution of vesicles appears to be unaltered (Fig.3.10 M). Moreover, no differences in the mean outer vesicle diameter (control: $44.60 \pm 0.385, n=21$; Syntaxin-1A KO/ $-B^{\text {YFP }}$ : $45.31 \pm 0.539, n=23 / P=0.2975$, n.s.; Fig. $3.10 \mathrm{~K}, \mathrm{~N}$ ) or in the mean vesicle volume were detected between the two groups (control: $46932 \pm 1193, n=21$; Syntaxin-1A KO/ $-B^{\text {YFP. }} 49567 \pm 1802, n=23 / P=0.2388$, n.s.; Fig. $3.10 \mathrm{~L})$.

\subsubsection{The role of the v-SNARE Synaptobevin-2 in synaptic vesicle docking}

Next, we decided to analyze the role of the v-SNARE Synaptobrevin-2 in synaptic vesicle docking in my experimental setting. Previous studies showed a $90 \%$ reduction of the sucrose-evoked RRP size in Synaptobrevin-2 KO neurons, but no change in the number of docked vesicles in electron micrographs (Deák et al., 2004; Schoch et al., 2001). However, a recent morphological study in PC12 cells expressing BoNT/D cleaving Synaptobrevin-1/2 indicated a role for the neuronal v-SNARE in large-dense core vesicle docking to the plasma membrane (Wu et al., 2012). These results are in contrast to previous findings that propose Synaptotagmin-1 as the molecular partner on vesicle membranes for LDCV docking, placing full SNARE-complex assembly downstream of docking (de Wit et al., 2009). 


\subsection{D-EM analysis of synaptic morphology in Synaptobrevin-2 KO neurons}

For the two-dimensional analysis, seven Synaptobrevin-2 $\mathrm{KO}$ animals $(\mathrm{N}=7)$ were compared with six littermate control animals $(\mathrm{N}=6)$, that were either wild-type or heterozygous for the Synaptobrevin-2 allele, from three cultures. Synaptobrevin-2 KO mice (Schoch et al., 2001) were purchased from 'The Jackson Laboratory'. The gross presynaptic morphology seemed to be unperturbed in Synaptobrevin-2 KO synaptic profiles analyzed in electron micrographs of ultrathin sections (Fig. 3.11 A, B). Neither the total number of synaptic vesicles per synaptic profile (control: $67.54 \pm 2.951, n=155$; Synaptobrevin-2 KO: $70.15 \pm 2.654, n=162$ / $P=0.4100$, n.s.; Fig. 3.11 C), nor the synaptic vesicle terminal density were different between the two groups (control: $1.733 \pm 0.050$, $\mathrm{n}=155$; Synaptobrevin-2 KO: $1.652 \pm 0.043, \mathrm{n}=162$ / $\mathrm{P}=0.2223$, n.s.; Fig. $3.11 \mathrm{D})$. Surprisingly, I observed an increase in PSD length in Synaptobrevin-2 KO synapses (control: $386.2 \pm 10.08, \mathrm{n}=155$; Synaptobrevin-2 KO: $426.9 \pm 8.674, \mathrm{n}=162$ / $\mathrm{P}<0.001$, ***; Fig. 3.11 F), which could only partially be explained by the observation that presynaptic terminals were slightly larger in Synaptobrevin-2 KO synapses (control: $398140 \pm 13958$, $\mathrm{n}=155$; Synaptobrevin-2 KO: $431227 \pm 13022, \mathrm{n}=162$ / $\mathrm{P}=0.0570$, n.s.; graph not shown). Moreover, a strong reduction in the synaptic vesicle cluster density for Synaptobrevin-2 KO synapses was observed (control: $4.525 \pm 0.055, n=155$; Synaptobrevin-2 KO: $4.065 \pm$ $0.050, \mathrm{n}=162 ; \mathrm{P}<0.001,{ }^{* *}$; Fig. $\left.3.11 \mathrm{E}\right)$, correlating with an increase in the number of larger, vesicular endosomal structures in presynaptic terminals of Synaptobrevin-2 KO synapses (control: $1.297 \pm 0.172, n=155$; Synaptobrevin-2 KO: $4.648 \pm 0.380, n=162$; $\mathrm{P}<0.001,{ }^{* *}$; Fig. $3.11 \mathrm{G}$ ). Additionally, I observed a dramatic increase in the number of LDCVs per synaptic profile in synapses lacking Synaptobrevin-2 (control: $0.290 \pm 0.057$, $\mathrm{n}=155$; Synaptobrevin-2 KO: $0.661 \pm 0.080, \mathrm{n}=162 ; \mathrm{P}<0.001$, ${ }^{* *}$; Fig. $3.11 \mathrm{H}$ ).

\subsection{D-ET analysis of synaptic vesicle docking in Synaptobrevin-2 KO neurons}

Samples from four Synaptobrevin-2 KO mice $(\mathrm{N}=4)$ and four control mice $(\mathrm{N}=4)$, from two independent cultures, were compared by electron tomographic analyisis (Fig. 3.12). This analysis revealed a strong reduction in the number of membrane-attached vesicles $(0-2$ $\mathrm{nm}$; control: $1.080 \pm 0.136, \mathrm{n}=25$; Synaptobrevin-2 KO: $0.355 \pm 0.099, \mathrm{n}=24 ; \mathrm{P}<0.001$, ***; Fig. $3.13 \mathrm{C})$ and closely tethered vesicles $(0-4 \mathrm{~nm}$; control: $1.46 \pm 0.161, \mathrm{n}=25$; Synaptobrevin-2 KO: $0.629 \pm 0.123, \mathrm{n}=24$ / $\mathrm{P}<0.001,{ }^{* * *}$; Fig. $\left.3.13 \mathrm{D}\right)$ in Synaptobrevin-2 
$\mathrm{KO}$ synapses compared to controls. The number of membrane-proximal vesicles $(0-40$ $n$; control: $2.600 \pm 0.173, n=25$; Synaptobrevin-2 KO: $2.672 \pm 0.105, n=24$ / P=0.7286, n.s.) (Fig. $3.13 \mathrm{~B}$ ) as well as the number of vesicles within $100 \mathrm{~nm}$ distance of the active zone was unaltered (control: $6.451 \pm 0.338, n=25$; Synaptobrevin-2 KO: $5.909 \pm 0.197$, n=24 / $\mathrm{P}=0.1772$, n.s.; Fig. 3.13 A). Synapses lacking Synaptobrevin-2 thus showed reductions in the number of membrane-attached and closely tethered vesicles to $33 \%$ and $43 \%$ of controls, respectively. Similar to synapses that were completely devoid of Munc13s and SNAP25, terminals lacking the neuronal v-SNARE Synaptobrevin-2 exhibited a prominent accumulation of synaptic vesicles at a close distance to the presynaptic active zone membrane, with a peak frequency occurring at around 4-6 nm from the active zone (Fig. $3.13 \mathrm{G}$ ). Moreover, synaptic vesicles in Synaptobrevin-2 KO presynaptic terminals exhibited increased mean outer synaptic vesicle diameters (control: $45.81 \pm 0.450, \mathrm{n}=25$; Synaptobrevin-2 KO: $49.07 \pm 0.411$, n=24 / P<0.001, ${ }^{* * *}$; Fig. $3.13 \mathrm{E}$, $\mathrm{H}$ ) with a corresponding increase in the mean synaptic vesicle volume (control: $51115 \pm$ 1549, n=25; Synaptobrevin-2 KO: $62908 \pm 1599, \mathrm{n}=24$ / $\mathrm{P}<0.0001$, ${ }^{* * *}$; Fig 3.13 F). These data indicate an increase in the mean vesicle diameter by $7 \%$ with a corresponding $25 \%$ increase in vesicle volume.

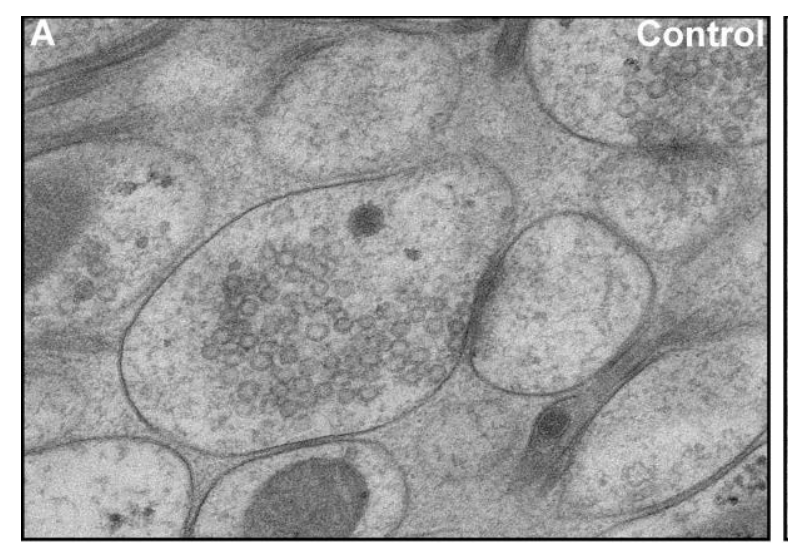

C

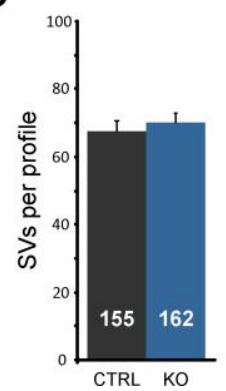

D

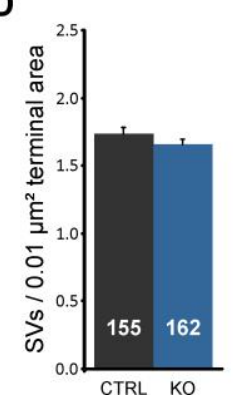

E

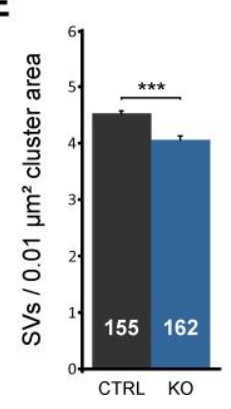

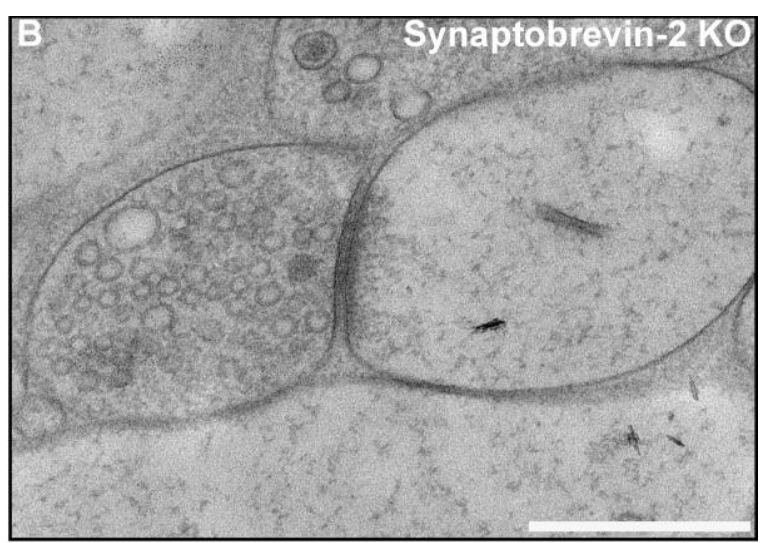

F

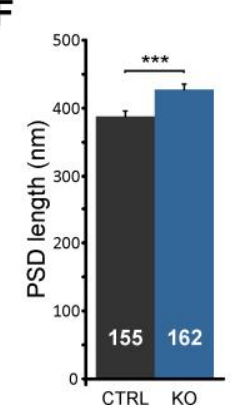

G

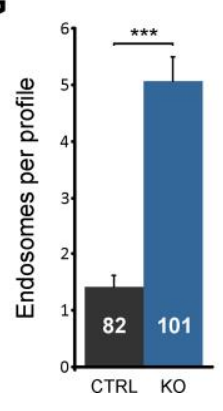

H

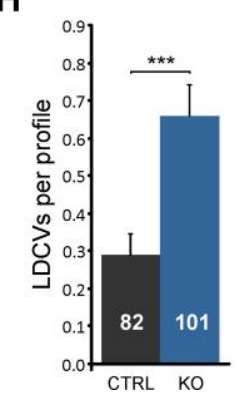


Figure 3.11. Two-dimensional ultrastructural analysis of synaptic morphology in Synaptobrevin-2 KO neurons

Electron micrographs of control (A) and Synaptobrevin-2 KO synaptic profiles (B) acquired from 60 $\mathrm{nm}$-thick ultrathin sections. Mean number of synaptic vesicles (SVs) per synaptic profile (C). Mean number of SVs normalized to synaptic terminal area (SV terminal density; D). Mean number of SVs normalized to SV cluster area (SV cluster density; E). Mean postsynaptic density (PSD) length (F) Mean number of endosomes per synaptic profile $(\mathbf{G})$. Mean number of large dense-core vesicles (LDCVs) per synaptic profile (H). C-H: Control: $\mathrm{N}=6, \mathrm{n}=155$; Synaptobrevin-2 KO: N=7, $\mathrm{n}=162$ (Mean + SEM), $\mathrm{P}<0.001:^{* \star *} ; \mathrm{P}<0.01:{ }^{* *} ; \mathrm{P}<0.05:{ }^{*}$. Scale bar: B, $500 \mathrm{~nm}$.

However, when comparing the individual docking profiles from Synaptobrevin-2 KO tomograms, I noticed that 8 out of 24 KO synapses (33\%) had similar number of membrane-attached vesicles $(0-2 \mathrm{~nm})$ as control synapses, whereas the remaining 16 synapses (67\%) had few or no docked vesicles (Fig. $3.13 \mathrm{I}$ ). I termed the synaptic profiles with normal synaptic vesicle docking $\mathrm{KO}^{\text {Plus }}$ (Fig. 3.13 C, F, I), and the synapses that are almost completely devoid of docked synaptic vesicles $\mathrm{KO}^{\text {Minus }}$ (Fig. 3.13 B, E, H).
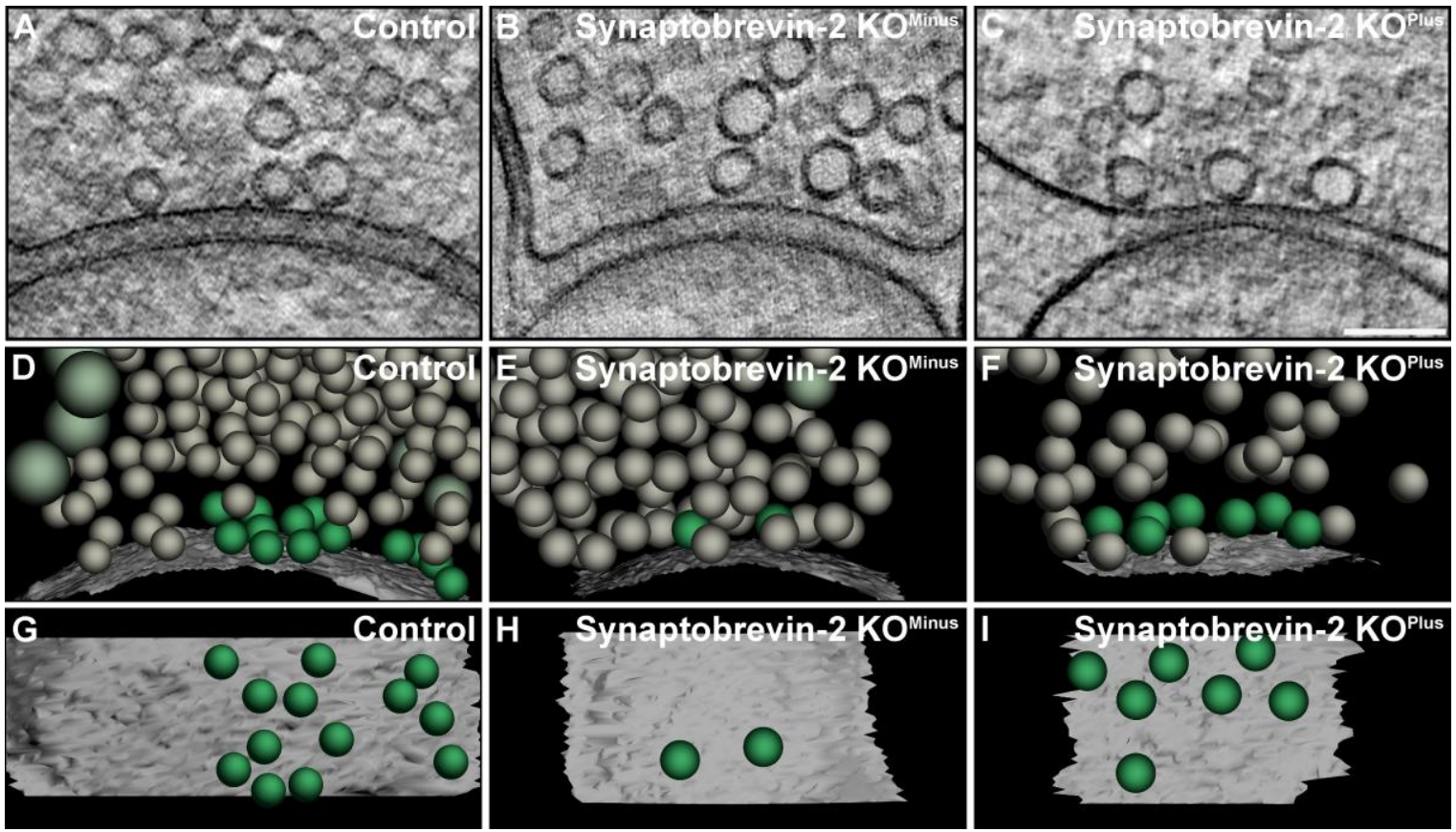

Figure 3.12. Three-dimensional electron tomographic analysis of synaptic vesicle docking in Synaptobrevin-2 KO neurons

Tomographically reconstructed subvolumes of control (A), Synaptobrevin $\mathrm{KO}^{\text {Minus }}$ (B) and Synaptobrevin $\mathrm{KO}^{\text {Plus }}$ (C) synapses from $200 \mathrm{~nm}$-thick sections. Three-dimensional models of tomographically reconstructed control (D) Synaptobrevin KO ${ }^{\text {Minus }}(\mathbf{E})$ and Synaptobrevin KO ${ }^{\text {Plus }}(\mathbf{F})$ synaptic profiles. Ultrastructural features reconstructed in the models include the active zone plasma membrane (white), synaptic vesicles (membrane-attached, green; non-attached, grey) and endosomes (light blue). Orthogonal views of control $(\mathbf{G})$ Synaptobrevin $\mathrm{KO}^{\text {Minus }}(\mathbf{H})$ and Synaptobrevin $\mathrm{KO}^{\text {Plus }}$ (I) tomographic models displaying the spatial arrangement of membraneattached synaptic vesicles within the reconstructed active zone area. Scale bar: B, $100 \mathrm{~nm}$. 
The number of membrane-attached synaptic vesicles $(0-2 \mathrm{~nm})$ was unchanged between control and $\mathrm{KO}^{\text {Plus }}$ synaptic profiles (control: $1.080 \pm 0.136, \mathrm{n}=25$; Synaptobrevin-2 $\mathrm{KO}$ Plus: $0.968 \pm 0.123, n=8 / P=0.6576$, n.s.; Fig. $3.13 \mathrm{~J}$ ). However, the number of docked synaptic vesicles $(0-2 \mathrm{~nm})$ was dramatically reduced when comparing control with $\mathrm{KO}{ }^{\text {Minus }}$ synaptic profiles (control: $1.080 \pm 0.136, n=25$; Synaptobrevin-2 KO ${ }^{\text {Minus: }} 0.049 \pm 0.022, n=16$ / $\left.\mathrm{P}<0.001,{ }^{* * *}\right)$ and $\mathrm{KO}^{\text {Plus }}$ with $\mathrm{KO}^{\text {Minus }}$ synaptic profiles ( $\mathrm{KO}^{\text {Plus: }} 0.968 \pm 0.123, \mathrm{n}=8$; Synaptobrevin-2 KOMinus: $0.049 \pm 0.022, n=16 / P<0.001$, ${ }^{* * *}$; Fig. $\left.3.13 \mathrm{~J}\right)$. In comparison to control and $\mathrm{KO}^{\text {Plus }}$ samples, $\mathrm{KO}^{\text {Minus }}$ synaptic profiles exhibited a reduction in the number of membrane-attached vesicles to $5 \%$.

To rule out the possibility that an increase in vesicle diameter could cause the reduced ability of synaptic vesicles to become attached to the presynaptic active zone membrane, I compared the mean outer synaptic vesicle diameter between control and $\mathrm{KO}^{\text {Plus }}$ (control: $45.81 \pm 0.45, n=25$; Synaptobrevin-2 KO Plus: $\left.48.65 \pm 0.54, n=8 / P=0.0023,{ }^{* *}\right)$ as well as between control and $\mathrm{KO}^{\text {Minus }}$ synaptic profiles (control: $45.81 \pm 0.4503, \mathrm{n}=25$; Synaptobrevin-2 KO $\left.{ }^{\text {Minus: }} 49.28 \pm 0.558, n=16 / \mathrm{P}=<0.001,{ }^{* * *}\right)$. I detected a significant increase in the vesicle diameter for Synaptobrevin-KO synapses in both cases, though with a slightly stronger phenotype for $\mathrm{KO}^{\text {Minus }}$ synaptic profiles (Fig. $3.13 \mathrm{~K}$ ). The mean outer synaptic vesicle diameters of $\mathrm{KO}^{\text {Plus }}$ and $\mathrm{KO}^{\text {Minus }}$ synaptic profiles were not statistically different (Synaptobrevin-2 KO Plus: $48.65 \pm 0.540, n=8$; Synaptobrevin-2 $\mathrm{KO}^{\text {Minus: }} 49.28 \pm 0.558, \mathrm{n}=16 ; \mathrm{P}=0.4822$, n.s.; Fig. $3.13 \mathrm{~K}, \mathrm{~N}$ ). I observed the same tendencies for the mean synaptic vesicle volume, which was significantly increased in $\mathrm{KO}^{\text {Plus }}$ synaptic profiles compared to controls (control: $51115 \pm 1549, \quad \mathrm{n}=25$; Synaptobrevin-2 KO Plus: $\left.61097 \pm 2125, n=8 / P=0.0023,{ }^{* *}\right)$ and in $K O^{\text {Minus }}$ synaptic profiles compared to controls (control: $51115 \pm 1549, \mathrm{n}=25$; Synaptobrevin-2 KO Minus: $63814 \pm$ $2160, \mathrm{n}=16 / \mathrm{P}<0.001,{ }^{* * *}$; Fig. $3.13 \mathrm{~L}$ ). The vesicle volume measured in $\mathrm{KO}^{\text {Plus }}$ and $\mathrm{KO}^{\text {Minus }}$ synaptic profiles was not statistically different (Synaptobrevin-2 KO Plus: $61097 \pm$ 2125, $n=8$; Synaptobrevin-2 KO ${ }^{\text {Minus: }} 63814 \pm 2160, n=16$ / P=0.4554, n.s.; Fig. 3.13 L).

When comparing the synaptic vesicle distribution within $100 \mathrm{~nm}$ distance of the active zone, $\mathrm{KO}^{\text {Minus }}$ synaptic profiles have almost no membrane-attached vesicles $(0-2 \mathrm{~nm})$, whereas both, the $\mathrm{KO}^{\text {Plus }}$ and $\mathrm{KO}^{\text {Minus }}$ groups, have a strong accumulation of membraneproximal vesicles. These synaptic vesicles accumulate in a close distance to the presynaptic active zone membrane, with a peak frequency at around 4-6 nm distance from the active zone (Fig. 3.13 M). I therefore exclude the possibility that an increase in synaptic vesicle size is responsible for the observed reduction in membrane-attached vesicles in Synaptobrevin-2 KO synapses. 
A

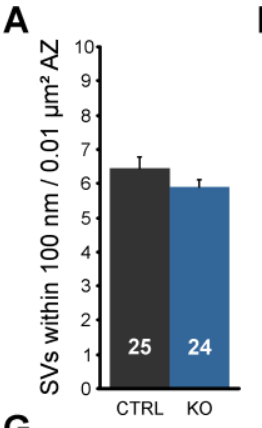

G

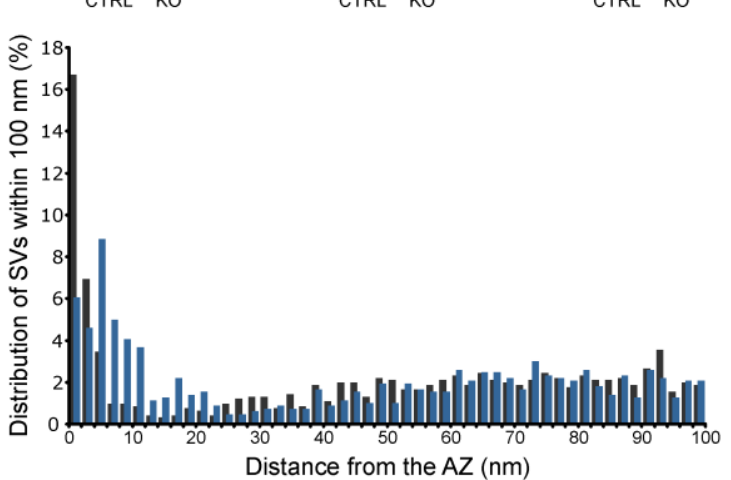

I

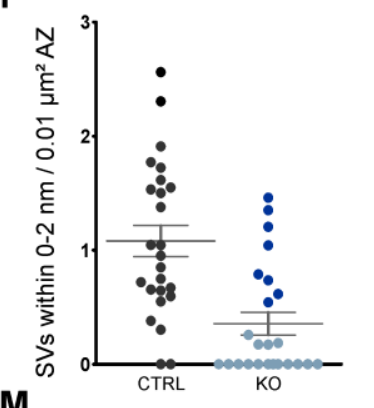

M
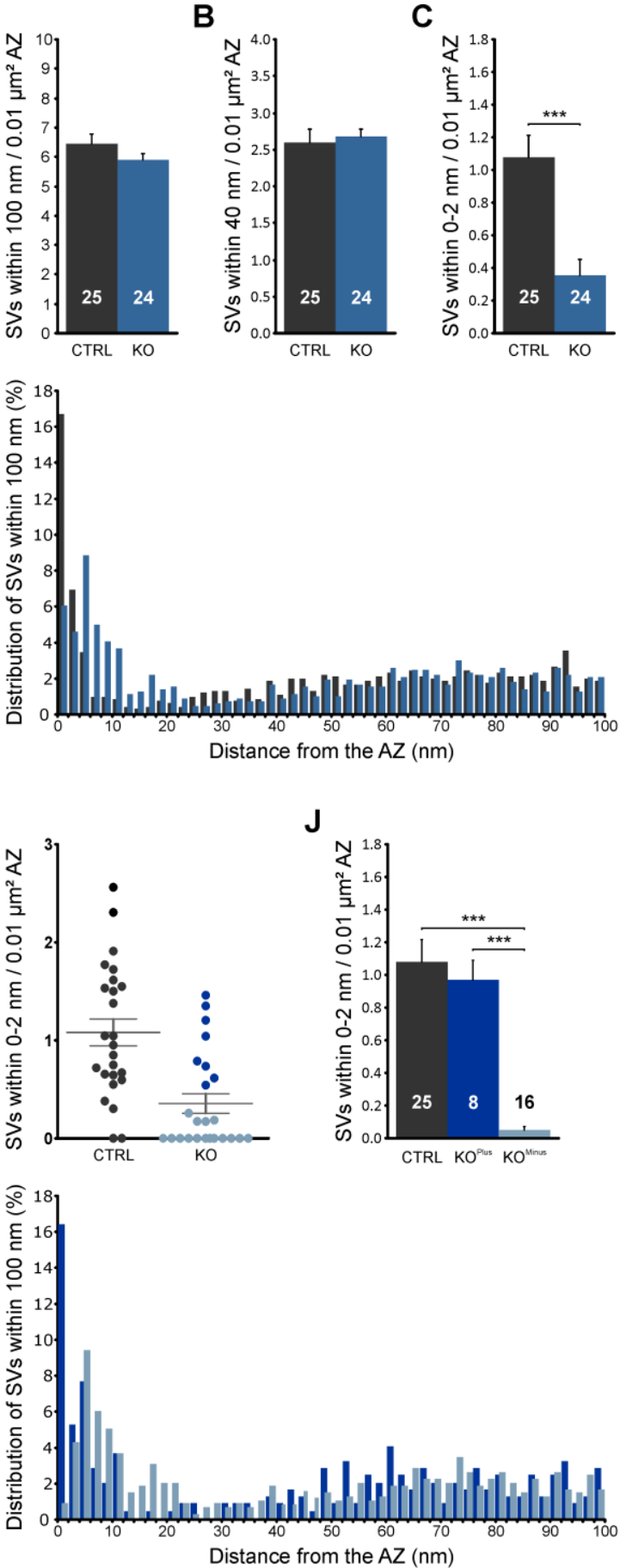

D

H

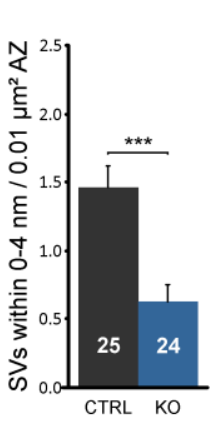

E
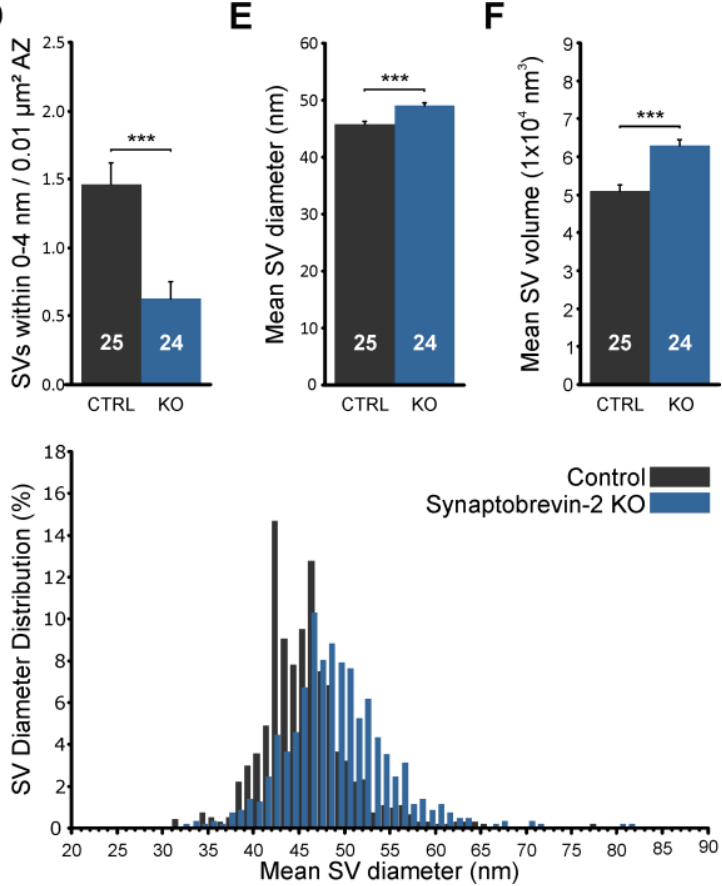

K

$\mathbf{N}$

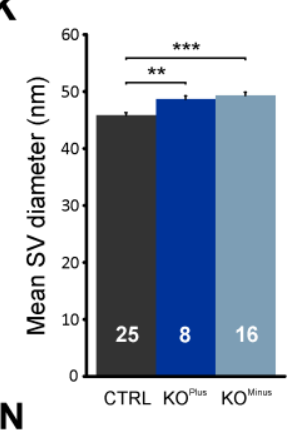

L

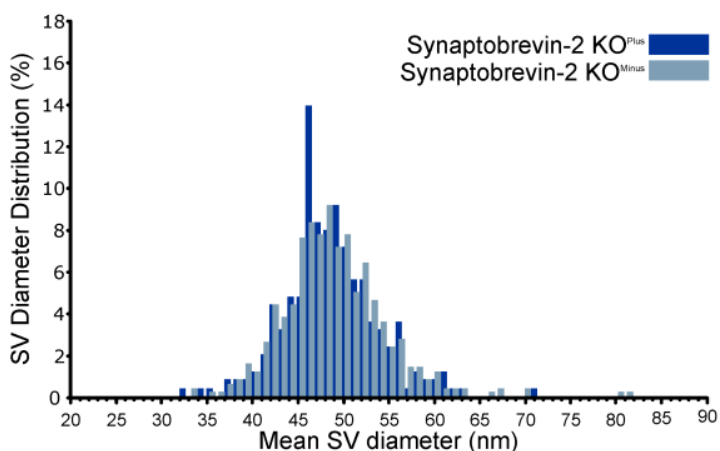

Figure 3.13. Quantitative analysis of synaptic vesicle docking in Synaptobrevin-2 KO neurons

Mean number of SVs within $100 \mathrm{~nm}$ of the $A Z$ normalized to $A Z$ area $(\mathbf{A})$. Mean number of SVs within $40 \mathrm{~nm}$ of the $A Z$ normalized to $A Z$ area (B). Mean number of membrane-attached SVs (within 0-2 $\mathrm{nm}$ of the $A Z$ ) normalized to $A Z$ area (C). Mean number of membrane-attached and closely-tethered SVs (within 0-4 $\mathrm{nm}$ of the AZ) normalized to AZ area (D). Mean outer SV diameter (F). Mean SV volume including the membrane bilayer (E). Spatial distribution of SVs within a 100 $\mathrm{nm}$ distance of the AZ membrane $(\mathbf{G})$. Distribution of synaptic vesicle diameters of vesicles within $100 \mathrm{~nm}$ of the $A Z(\mathbf{H})$. Scatterplot showing number of membrane-attached SVs $(0-2 \mathrm{~nm})$ normalized to $A Z$ area in individual tomograms from control and Synaptobrevin-2 KO synaptic profiles (I). Mean number of membrane-attached SVs (within 0-2 nm of the AZ) normalized to AZ 
area comparing control, Synaptobrevin-2 $\mathrm{KO}^{\text {Plus }}$ and Synaptobrevin-2 $\mathrm{KO}^{\text {Minus }}$ synaptic profiles (J). Mean outer SV diameter from control, Synaptobrevin-2 KO ${ }^{\text {Plus }}$ and Synaptobrevin-2 KO Minus synaptic profiles (K). Mean SV volume including the membrane bilayer from control, Synaptobrevin-2 KO $\mathrm{KO}^{\text {Plus }}$ and Synaptobrevin-2 $\mathrm{KO}^{\text {Minus }}$ synaptic profiles (L). Spatial distribution of SVs within a $100 \mathrm{~nm}$ distance of the $\mathrm{AZ}$ membrane comparing Synaptobrevin-2 $\mathrm{KO}^{\text {Plus }}$ and Synaptobrevin-2 KO ${ }^{\text {Minus }}$ synaptic profiles $(\mathbf{M})$. Distribution of synaptic vesicle diameters of vesicles within $100 \mathrm{~nm}$ of the AZ comparing Synaptobrevin-2 KO ${ }^{\text {Plus }}$ and Synaptobrevin-2 KO ${ }^{\text {Minus }}$ synaptic profiles $(\mathbf{N})$.

A-F: Control: $\mathrm{N}=4, \mathrm{n}=25$; Synaptobrevin-2 KO: $\mathrm{N}=4, \mathrm{n}=24$ (Mean + SEM), $\mathrm{P}<0.001$ : $^{* * *}$; $\mathrm{P}<0.01$ : ${ }^{* *}$; $\mathrm{P}<0.05:{ }^{*}$. G, H: (890 SVs in control and $753 \mathrm{SVs}$ in Synaptobrevin-2 KO synaptic profiles). J-L: Control: $N=4, n=25$; Synaptobrevin-2 KO ${ }^{\text {Plus: }} \mathrm{N}=4, \mathrm{n}=8$; Synaptobrevin-2 $\mathrm{KO}^{\text {Minus }}: \mathrm{N}=4, \mathrm{n}=16$ (Mean + SEM), $\mathrm{P}<0.001$ : ${ }^{* *} ; \mathrm{P}<0.01$ : ${ }^{* *} ; \mathrm{P}<0.05:{ }^{*} . \mathbf{M}, \mathbf{N}$ : (251 SVs in Synaptobrevin-2 KO ${ }^{\text {Plus }}$ and 502 SVs in Synaptobrevin-2 KO Minus synaptic profiles). Scale bar: B, $100 \mathrm{~nm}$.

J-L: Control: $N=4, n=25$; Synaptobrevin-2 KO ${ }^{\text {Plus }}: N=4, n=8$; Synaptobrevin-2 KO ${ }^{\text {Minus }}: N=4, n=16$ (Mean + SEM), $P<0.001:{ }^{* \star *} ; \mathrm{P}<0.01{ }^{* *} ; \mathrm{P}<0.05:{ }^{*} . \mathbf{G}, \mathbf{H}:\left(251 \mathrm{SV}\right.$ s in Synaptobrevin-2 KO${ }^{\text {Plus }}$ and 502 SVs in Synaptobrevin-2 KO ${ }^{\text {Minus }}$ synaptic profiles).

\subsection{Synaptobrevin-1 can compensate for the loss of Synaptobrevin-2 in synapses}

In order to explain the presence of membrane-attached synaptic vesicles in a subset of Synaptobrevin-2 $\mathrm{KO}$ synapses ( $\mathrm{KO}^{\text {Plus }}$ ), we scrutinized the localization pattern of immunolabeled Synaptobrevin-1 to reveal any potential compensatory changes in organotypic slices lacking Synaptobrevin-2. In an earlier study, Synaptobrevin-1 was not detected by Western blot analysis of PO Synaptobrevin-2 KO mouse brain (Schoch et al., 2001). However, in situ hybridization data indicate that expression of Synaptobrevin-1 in the hippocampus starts in vivo at around P14 (Allen Developing Mouse Brain Atlas). Immunohistochemistry experiments and the imaging analysis were performed by Dr. Benjamin Cooper, Department of Molecular Neurobiology of the Max-Planck Institute of Experimental Medicine, Göttingen, Germany. Organotypic slices from two wild-type and two Synaptobrevin-2 KO animals were fixed after 4-5 weeks in culture, processed for immunohistochemistry and labeled by using isoform-specific antibodies against Synaptobrevin-2, Synaptobrevin-1 and VGLUT1. Wild-type organotypic slices exhibited a specific punctate labeling of Synaptobrevin-2, which highly co-localized with VGLUT1, but was not detectable in Synaptobrevin-2 KO cultures (data not shown). In wild-type slices, Synaptobrevin-1 labeling revealed the presence of large immunoreactive puncta in organotypic slices (Fig. S2 A). However, only a subset ( 8\%) of VGLUT1 positive puncta colocalized with the Synaptobrevin-1 signal (Fig. S2 B, C). These findings indicate that in control slices, Synaptobrevin-1 is predominantly localized to nonglutamatergic synapses. Synaptobrevin-2 KO organotypic slices exhibited an increase in the number of Synaptobrevin-1 positive puncta, contributed by an increase in the frequency of low 
intensity Synaptobrevin-1 immunoreactive structures (control: $1 \pm 0.03910, n=27$; Synaptobrevin-2 KO: $2.738 \pm 0.229, \mathrm{n}=27$ / $\mathrm{P}<0.001$, ${ }^{* * *}$; Fig. S2 D, G). In comparison to control slices, the relative number of VGLUT1 positive puncta was slightly increased in Synaptobrevin-2 KO cultures (control: $1 \pm$ 0.0286, n=27; Synaptobrevin-2 KO: $1.195 \pm$ 0.030, $\mathrm{n}=27$ / $\mathrm{P}<0.001,{ }^{* * *}$; Fig. S2 H). Similar to SNAP25 KO organotypic slices, cultures devoid of Synaptobrevin-2 exhibited a significant increase in the size of VGLUT1 immunoreactive puncta (control: $1 \pm 0.030, \mathrm{n}=27$; Synaptobrevin-2 KO: $1.577 \pm 0.068$, $\mathrm{n}=27 / \mathrm{P}<0.001,{ }^{\star * *}$; Fig. I). Interestingly, we found an increase in the degree of colocalization for VGLUT1 and Synaptobrevin-1 in Synaptobrevin-2 KO slices (Fig. S2 E, F). In Synaptobrevin-2 KO slices, 36\% of VGLUT1 positive puncta were immunoreactive for Synaptobrevin-1, indicating that Synaptobrevin-1 expression is upregulated in glutamatergic synapses in the absence of Synaptobrevin-2 (control: $7.739 \pm 1.459, n=27$; Synaptobrevin-2 KO: $36.39 \pm 2.046, \mathrm{n}=27$ / $\mathrm{P}<0.001,{ }^{* * *}$; Fig. S2 J).

In summary, mature organotypic hippocampal slices deficient of Synaptobrevin-2 exhibit a compensatory increase in the number of Synaptobrevin-1 positive glutamatergic, excitatory synapses, potentially explaining the observation that $33 \%$ of all sampled synaptic profiles in the electron tomographic analysis of synaptic vesicle docking still had membrane-attached synaptic vesicles. However, whether or not Synaptobrevin-1 can rescue the physiological deficits seen in Synaptobrevin-2 deficient neurons has not yet been assessed to my knowledge.

\subsubsection{The role of the vesicular $\mathrm{Ca}^{2+}$-sensor Synaptotagmin-1 in synaptic vesicle docking}

Next, I studied the function of Synaptotagmin-1 in synaptic vesicle docking. Synaptotagmin-1, the exocytotic $\mathrm{Ca}^{2+}$-sensor, has also been implicated in secretory and synaptic vesicle docking, and in the regulation of total synaptic vesicle numbers, which are reduced in Synaptotagmin-1 KO animals (Jorgensen et al., 1995; Liu et al., 2009; de Wit et al., 2009). In-vitro data from liposome fusion assays indicated a role for Synaptotagmin1 as a distance-regulator for synaptic vesicle fusion, keeping plasma membrane and vesicle membrane apart to prevent SNARE-complex formation and fusion prior to $\mathrm{Ca}^{2+}$ influx. $\mathrm{Ca}^{2+}$-binding to the $\mathrm{C}_{2}$ domains of Synaptotagmin-1 would then trigger SNAREnucleation and membrane fusion in a post-docking step (van den Bogaart et al., 2011). In vivo, the role of Synaptotagmin-1 in synaptic vesicle docking in neurons remains a point of heavy discussion, a confounding factor being the variety of model systems, fixation 
protocols (chemical vs. cryo-fixation), data acquisition methods (two-dimensional versus three-dimensional EM analyses), and above all, differences in "docking" definitions used to study membrane-attachment by EM (discussed in: Verhage and Sørensen 2008).

\subsection{D-EM analysis of synaptic morphology in Synaptotagmin-1 KO neurons}

For the two-dimensional analysis of basic ultrastructural features in the absence of Synaptotagmin-1, samples from four Synaptotagmin-1 KO animals $(\mathrm{N}=4)$ were compared with samples from five control animals $(\mathrm{N}=5)$, that were either wildtype or heterozygous for the Synaptotagmin-1 KO allele. No major morphological changes between control and Synaptotagmin-1 KO synaptic profiles were observed in electron micrographs (Fig. 3.14 $A, B)$. First, no changes in the overall number of synaptic vesicles per synaptic profile were detected between genotypes (control: $52.8 \pm 2.573, \mathrm{n}=114$; Synaptotagmin-1 KO: $50.3 \pm 2.608, n=97 / P=0.4952$, n.s.; Fig. 3.14 C). However, when I normalized the number of synaptic vesicles to the area of the synaptic terminal I could unmask a small but significant reduction in the terminal vesicle density in Synaptotagmin-1 KO synapses (control: $1.595 \pm 0.05415, \mathrm{n}=114$; Synaptotagmin-1 KO: $1.363 \pm 0.055, \mathrm{n}=97 / \mathrm{P}=$ $0.0045,{ }^{* *}$; Fig. $3.14 \mathrm{D}$ ), which was also detected as a reduction of the synaptic vesicle cluster density (control: $3.946 \pm 0.063, \mathrm{n}=114$; Synaptotagmin-1 KO: $3.658 \pm 0.062, \mathrm{n}=$ $97 / P=0.0034,{ }^{* *}$; Fig. $3.14 \mathrm{E}$ ). No differences in the PSD length (control: $333.4 \pm 10.03$, $\mathrm{n}=114$; Synaptotagmin-1 KO: $345.0 \pm 11.67, \mathrm{n}=97$ / $\mathrm{P}=0.3661$, n.s.; Fig. $3.14 \mathrm{~F}$ ) in the number of endosomes per synaptic profile (control: $0.211 \pm 0.046, n=114$; Synaptotagmin-1 KO: $0.175 \pm 0.046, n=97 / P=0.046$; Fig. $3.14 \mathrm{G}$ ), or in the number of LDCVs per synaptic profile were detected (control: $0.211 \pm 0.046, \mathrm{n}=114$; Synaptotagmin-1 KO: $0.175 \pm 0.046, n=97 / P=0.5481$, n.s.; Fig. $3.14 \mathrm{H}$ ).

\subsection{D-ET analysis of synaptic vesicle docking in Synaptotagmin-1 KO neurons}

For the three-dimensional electron tomographic analysis of synaptic vesicle docking in the absence of Synaptotagmin-1, samples from three Synaptotagmin-1 KO animals $(\mathrm{N}=3)$ were compared with samples from four control animals $(\mathrm{N}=4)$, that were either wildtype or heterozygous for the Synaptotagmin-1 KO allele (Fig. 3.15 A-F). Synaptotagmin-1 KO profiles exhibited reductions in the number of membrane-attached synaptic vesicles (0-2 
$\mathrm{nm}$; control: $1.494 \pm 0.203, \mathrm{n}=23$; Synaptotagmin-1 KO: $0.906 \pm 0.156, \mathrm{n}=20 / \mathrm{P}=$ $0.0302,{ }^{*}$; Fig. $\left.3.15 \mathrm{I}\right)$ and in the number of closely tethered synaptic vesicles $(0-4 \mathrm{~nm}$; control: $2.062 \pm 0.185, \mathrm{n}=23$; Synaptotagmin-1 KO: $1.433 \pm 0.217, \mathrm{n}=20 / \mathrm{P}=0.0319$, *; Fig. $3.15 \mathrm{~J})$. In contrast to all previously analyzed mouse mutants, I detected a significant decrease in the number of membrane-proximal synaptic vesicles $(0-40 \mathrm{~nm})$ in synapses lacking Synaptotagmin-1 in comparison to control synaptic profiles (control: $3.630 \pm 0.243$, $\mathrm{n}=23$; Synaptotagmin-1 KO: $2.802 \pm 0.289, \mathrm{n}=20$ / $\mathrm{P}=0.0329$, *; Fig. $3.15 \mathrm{H}$ ), whereas the number of vesicles within $100 \mathrm{~nm}$ distance of the active zone was only slightly, but not significantly, reduced (control: $8.997 \pm 0.644, n=23$; Synaptotagmin-1 KO: $7.628 \pm 0.500$, $\mathrm{n}=20 / \mathrm{P}=0.1080$, n.s.; Fig. $3.15 \mathrm{G}$ ). Moreover, the synaptic vesicle distribution within $100 \mathrm{~nm}$ distance of the active zone revealed a reduction in the number of membraneattached synaptic vesicles, but did not show an accumulation of vesicles close to the active zone membrane as was observed for the KOs of the t-SNARE SNAP25 and of Munc13s (Fig. 3.15 M).
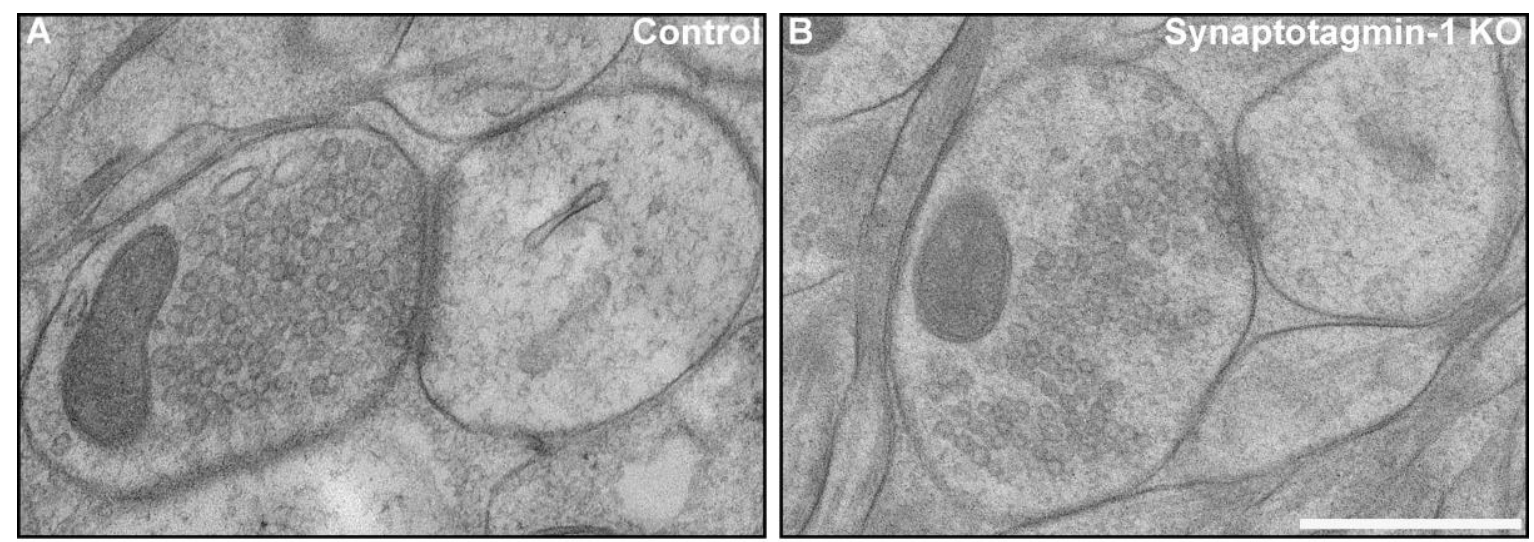

C

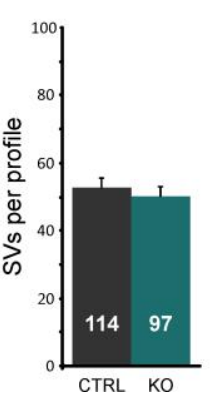

D

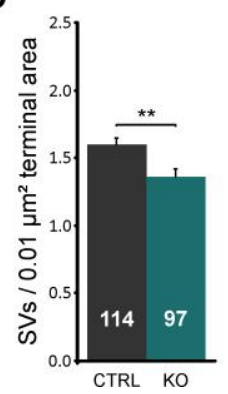

E

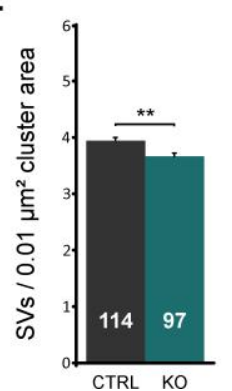

$\mathbf{F}$

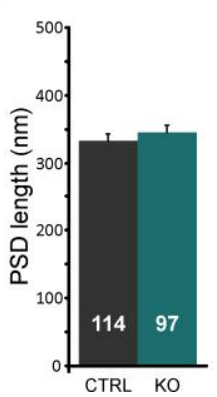

G

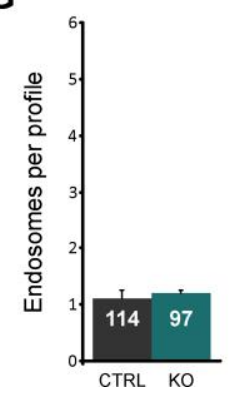

H

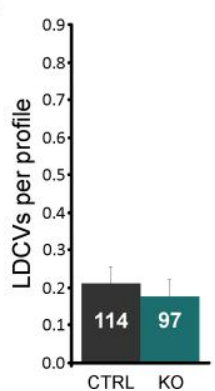

Figure 3.14. Two-dimensional ultrastructural analysis of synaptic morphology in Synaptotagmin-1 KO neurons

Electron micrographs of control (A) and Synaptotagmin-1 KO (B) synaptic profiles acquired from 60 $\mathrm{nm}$-thick ultrathin sections. Mean number of synaptic vesicles (SVs) per synaptic profile (C). Mean number of SVs normalized to synaptic terminal area (SV terminal density; D). Mean number of SVs normalized to SV cluster area (SV cluster density; E). Mean postsynaptic density (PSD) length (F). Mean number of endosomes per synaptic profile $(\mathbf{G})$. Mean number of large dense-core vesicles (LDCVs) per synaptic profile (H). C-H: Control: $\mathrm{N}=5, \mathrm{n}=114$; Synaptotagmin-1 KO: $\mathrm{N}=4, \mathrm{n}=97$ (Mean + SEM), $P<0.001:{ }^{* * *} ; \mathrm{P}<0.01:^{* *} ; \mathrm{P}<0.05:{ }^{*}$. Scale bar: B, $500 \mathrm{~nm}$. 

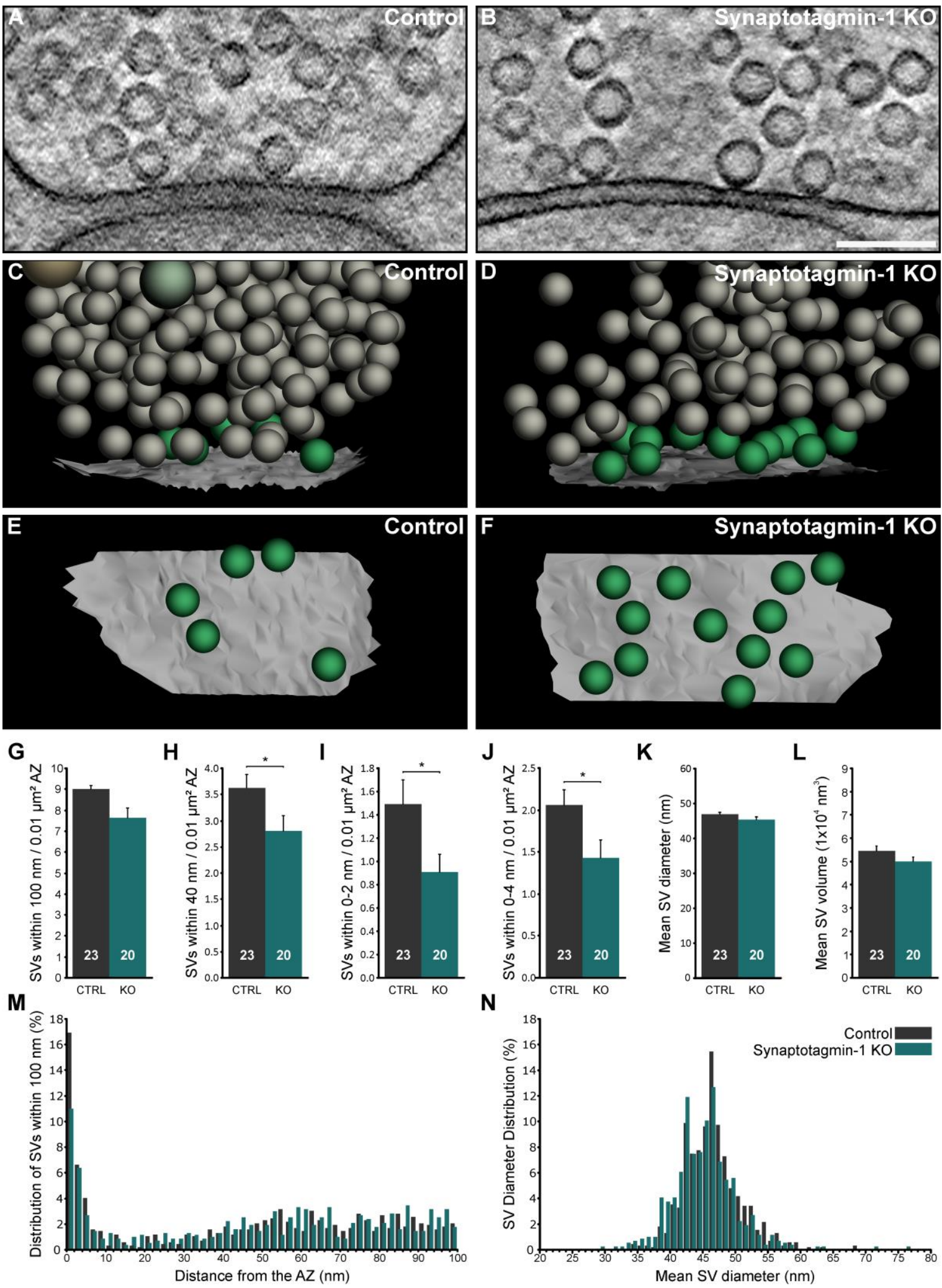

Figure 3.15. Three-dimensional electron tomographic analysis of synaptic vesicle docking in Synaptotagmin-1 KO neurons

Tomographically reconstructed subvolumes of control (A) and Synaptotagmin-1 KO (B) synapses from $200 \mathrm{~nm}$-thick sections. Three-dimensional models of tomographically reconstructed control (C) and Synaptotagmin-1 KO (D) synaptic profiles. Ultrastructural features reconstructed in the models include the active zone plasma membrane (white), synaptic vesicles (membrane-attached, green; non-attached, grey), large dense core vesicles (LDCVs, beige) and endosomes (light blue). 
Orthogonal views of control (E) and Synaptotagmin-1 KO (F) tomographic models displaying the spatial arrangement of membrane-attached synaptic vesicles within the reconstructed active zone area. Mean number of SVs within $100 \mathrm{~nm}$ of the $A Z$ normalized to $A Z$ area $(\mathbf{G})$. Mean number of SVs within $40 \mathrm{~nm}$ of the $A Z$ normalized to $A Z$ area $(\mathbf{H})$. Mean number of membrane-attached SVs (within 0-2 nm of the AZ) normalized to AZ area (I). Mean number of membrane-attached and closely-tethered SVs (within 0-4 nm of the AZ) normalized to AZ area ( $\mathbf{J}$ ). Mean outer SV diameter (K). Mean SV volume including the membrane bilayer (L). Spatial distribution of SVs within a 100 $\mathrm{nm}$ distance of the $\mathrm{AZ}$ membrane $(\mathbf{M})$. Distribution of synaptic vesicle diameters of vesicles within $100 \mathrm{~nm}$ of the AZ (N). G-L: Control: $\mathrm{N}=4, \mathrm{n}=23$; Synaptotagmin-1 KO: $\mathrm{N}=3, \mathrm{n}=20$ (Mean $+\mathrm{SEM})$, $\mathrm{P}<0.001:^{* * *} ; \mathrm{P}<0.01:^{* *} ; \mathrm{P}<0.05:{ }^{*} . \mathbf{M}, \mathbf{N}$ : (807 SVs in control and 650 SVs in Synaptotagmin-1 KO synaptic profiles). Scale bar: $\mathbf{B}, 100 \mathrm{~nm}$.

In comparison to control synaptic profiles, synapses devoid of Synaptotagmin-1 exhibited reductions in the number of membrane-attached and closely tethered vesicles to $61 \%$ and $70 \%$ of controls, respectively. The number of membrane-proximal vesicles within $40 \mathrm{~nm}$ of the active zone is reduced to $77 \%$ compared to control synaptic profiles. However, taking the reduced density of membrane proximal synaptic vesicles into consideration, the number of membrane-attached $(0-2 \mathrm{~nm})$ and closely tethered $(0-4 \mathrm{~nm})$ vesicles normalized to the total number of vesicles within $40 \mathrm{~nm}$ of the active zone show reductions to only $74 \%$ and $83 \%$ of controls, respectively. In addition to these findings, I did not observe any changes in the mean outer vesicle diameter (control: $46.81 \pm 0.548, n$ = 23; Synaptotagmin-1 KO: $45.41 \pm 0.582, n=20 / P=0.0873$, n.s.; Fig. $3.15 \mathrm{~K}, \mathrm{~N}$ ) or in the mean vesicle volume between the two groups (control: $54578 \pm 2011, n=23$; Synaptotagmin-1 KO: $49958 \pm 1941, n=20$ / $P=0.1086$, n.s.; Fig. 3.15 L).

In summary, Synaptotagmin-1 KO neurons exhibit a decrease in the number of membrane-attached synaptic vesicles, which points towards a regulatory function of Synaptotagmin-1 in synaptic vesicle docking.

\subsubsection{The role of Complexins in synaptic vesicle docking}

Finally, I included a Complexin-1/2/3 triple KO (TKO) mouse line into my analysis (Xue et al., 2008). Complexins are a family of proteins, which can bind with a central $\alpha$-helix to the assembled SNARE-complex. By this action, murine Complexin isoforms were previously shown to facilitate fast neurotransmitter release in a post-priming step ('super-priming'), since the EPSC amplitude and the vesicular release probability is drastically reduced in hippocampal neurons from Complexin-1/2/3 triple $\mathrm{KO}$ mice with no changes in the RRP size or in the number of docked vesicles (Reim et al., 2001; Xue et al., 2007, 2008). However, a recent study describes a drastic decrease in the RRP size after lentiviral 
knock-down of Complexin, proposing a priming role for Complexin upstream of Synaptotagmin-1 function and an additional role as a fusion clamp for asynchronous and spontaneous release in concert with Synaptotagmin-1 (Yang et al., 2010). Moreover, C. elegans Complexin mutants show a severe reduction in the number of docked vesicles, most likely due to an increase in spontaneous fusion events caused by the absence of Complexin-mediated inhibitory clamping-functions (Hobson et al., 2011).

\subsection{D-EM analysis of synaptic morphology in Complexin-1/2/3 TKO neurons}

Samples from three Complexin TKO animals (Complexin- $1^{-/}$, Complexin- $2^{-1-}$, Complexin- $3^{-/}$) were compared with three control animals with the following genotypes

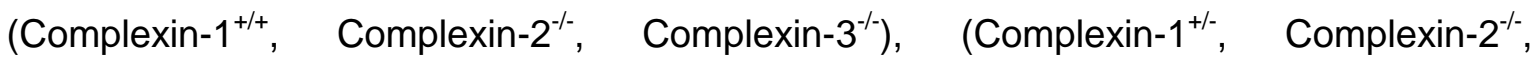
Complexin- $\left.3^{-/}\right)$, (Complexin- $1^{+-}$, Complexin- $2^{--_{-}}$, Complexin- $3^{+/}$). In the two-dimensional analysis of electron micrographs, no statistically significant differences were detected in any of the parameters measured (Fig. 3.16 A, B). The total number of synaptic vesicles per synaptic profile (control: $68.75 \pm 2.635, n=222$; Complexin-1/2/3 TKO: $67.66 \pm 3.241$, $\mathrm{n}=155 / \mathrm{P}=0.4543$, n.s.; Fig. $3.16 \mathrm{C}$ ), the synaptic vesicle terminal density (control: $1.539 \pm 0.037, n=222$; Complexin-1/2/3 TKO: $1.581 \pm 0.043, n=155$ / $P=0.4448$, n.s.; Fig. $3.16 \mathrm{D})$ and the synaptic vesicle cluster density were unchanged between the two groups (control: $3.883 \pm 0.038, n=222$; Complexin-1/2/3 TKO: $3.991 \pm 0.050, n=155 / P$ $=0.2399$, n.s.; Fig. 3.16 E). Moreover, the PSD length (control: $361.3 \pm 8.92, n=222$; Complexin-1/2/3 TKO: $378.2 \pm 12.22, n=155$ / $P=0.3383$, n.s.; Fig. 3.16 F), the number of endosomes per synaptic profile (control: $1.063 \pm 0.108, n=222$; Complexin-1/2/3 TKO: $1.103 \pm 0.121, n=155 / P=0.7002$, n.s.; Fig. $3.16 \mathrm{G}$ ) and the number of LDCVs per presynaptic profile did not differ between Complexin-1/2/3 TKO and control neurons (control: $0.176 \pm 0.031, \mathrm{n}=222$; Complexin-1/2/3 TKO: $0.219 \pm 0.047, \mathrm{n}=155 / \mathrm{P}=$ 0.6946, n.s.; Fig. $3.16 \mathrm{H}$ ). 


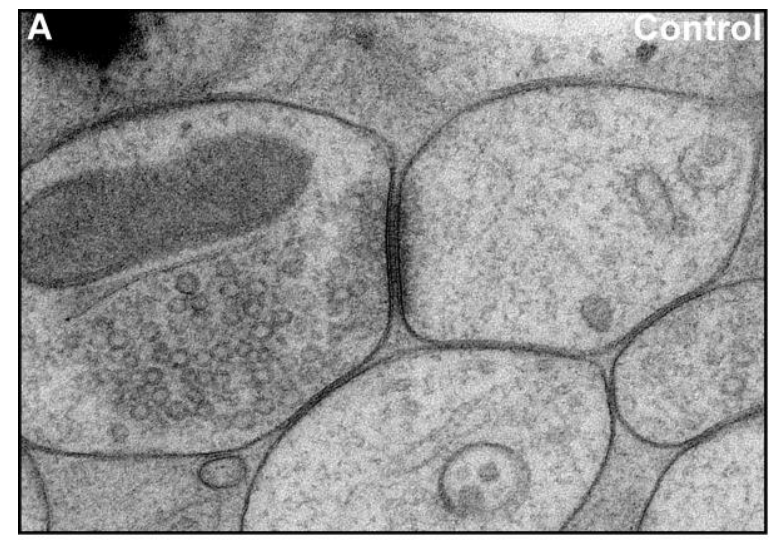

C

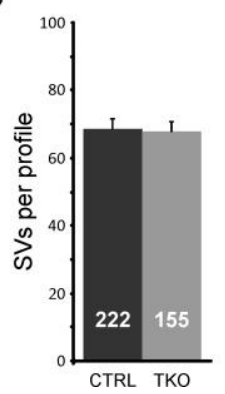

D

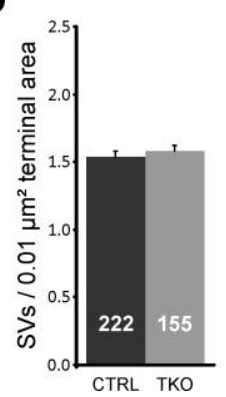

E

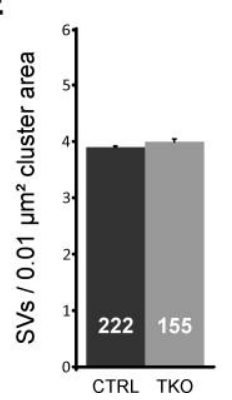

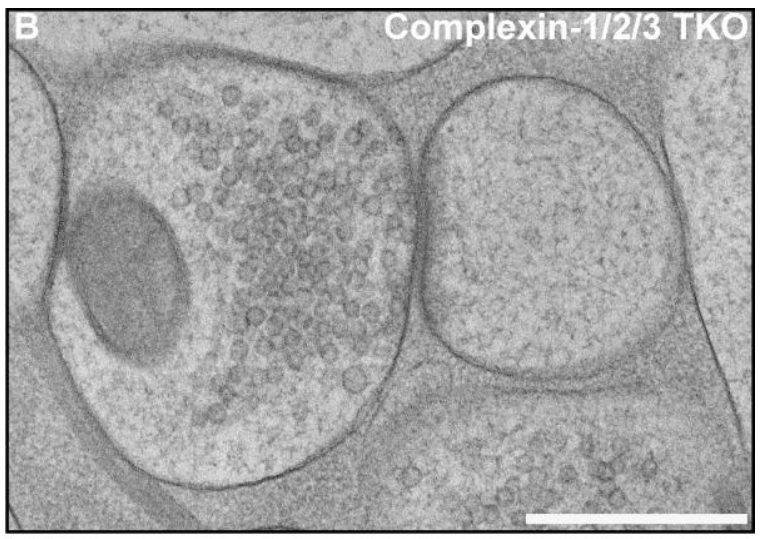

$\mathbf{F}$

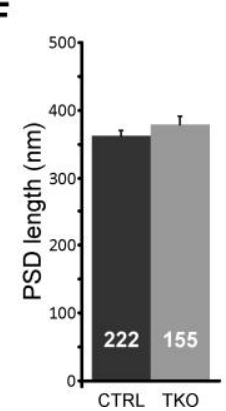

G

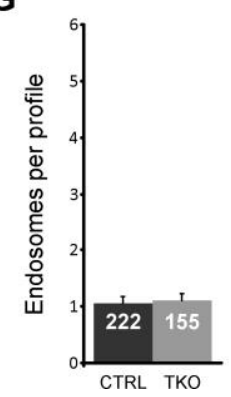

H

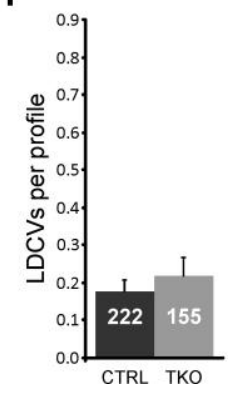

Figure 3.16. Two-dimensional ultrastructural analysis of synaptic morphology in Complexin1/2/3 TKO neurons

Electron micrographs of control (A) and Complexin-1/2/3 TKO (B) synaptic profiles acquired from $60 \mathrm{~nm}$-thick ultrathin sections. Mean number of synaptic vesicles (SVs) per synaptic profile (C). Mean number of SVs normalized to synaptic terminal area (SV terminal density; D). Mean number of SVs normalized to SV cluster area (SV cluster density; E). Mean postsynaptic density (PSD) length (F) Mean number of endosomes per synaptic profile $(\mathbf{G})$. Mean number of large dense-core vesicles (LDCVs) per synaptic profile (H). C-H: Control: $\mathrm{N}=3, \mathrm{n}=222$; Complexin-1/2/3 TKO: $\mathrm{N}=3$, $\mathrm{n}=155$ (Mean + SEM), $\mathrm{P}<0.001:^{* * *} ; \mathrm{P}<0.01:^{* *} ; \mathrm{P}<0.05:{ }^{*}$. Scale bar: B, $500 \mathrm{~nm} .{ }^{*}$. Imaging and analysis were performed by $\mathrm{S}$. Krinner.

\subsection{D-ET analysis of synaptic vesicle docking in Complexin-1/2/3 TKO neurons}

Next, I analyzed synaptic vesicle docking in presynaptic terminals from electron tomographic reconstructions of $200 \mathrm{~nm}$ semithin sections in glutamatergic, excitatory spine synapses of control $(\mathrm{N}=3)$ and Complexin-1/2/3 TKO samples ( $\mathrm{N}=3$ ) (Fig. 3.17 A-F). In three-dimensional electron tomograms, no significant changes were detected in the number of active zone membrane-attached synaptic vesicles $(0-2 \mathrm{~nm}$; control: $0.871 \pm$ 0.147, $n=19$; Complexin-1/2/3 TKO: $1.116 \pm 0.145, n=25$ / P = 0.248, n.s.; Fig. 3.17 I) or in the number of closely-tethered synaptic vesicles $(0-4 \mathrm{~nm}$; control: $1.219 \pm 0.168, \mathrm{n}=19$; Complexin-1/2/3 TKO: $1.602 \pm 0.159, n=25 / P=0.1586$, n.s.; Fig. $3.17 \mathrm{~J})$. However, there was a tendency towards a slightly higher number of membrane-attached vesicles in Complexin-1/2/3 TKO synapses. The number of membrane proximal vesicles within 40 
$\mathrm{nm}$ distance of the active zone did not differ between the two groups (control: $2.386 \pm$ 0.180, $n=19$; Complexin-1/2/3 TKO: $2.728 \pm 0.140, n=25$ / P = 0.135, n.s.; Fig. 3.17 H), although a slight increase in the number of vesicles within $100 \mathrm{~nm}$ of the active zone in Complexin-1/2/3 synaptic profiles was observed (control: $6.005 \pm 0.319, \mathrm{n}=19$; Complexin-1/2/3 TKO: $6.861 \pm 0.247, \mathrm{n}=25 / \mathrm{P}=0.0371$, *; Fig. $3.17 \mathrm{G})$. The synaptic vesicle distribution within $100 \mathrm{~nm}$ distance from the presynaptic active zone membrane revealed no major differences between control and Complexin-1/2/3 KO synaptic profiles, with the largest percentage of vesicles being membrane-attached in both groups (Fig. 3.17 M). Neither the mean outer synaptic vesicle diameter (control: $44.71 \pm 0.621, n=19$; Complexin-1/2/3 TKO: $44.11 \pm 0.407, \mathrm{n}=25 / \mathrm{P}=0.409$, n.s.; Fig. $3.17 \mathrm{~K}, \mathrm{~N}$ ) nor the mean synaptic vesicle volume were altered in synapses lacking all Complexin isoforms (control: $47600 \pm 2017, n=19$; Complexin-1/2/3 TKO: $45536 \pm 1198, n=25 / P=0.3596$, n.s.; Fig. 3.17 L).

In summary, I could not detect any statistically significant differences in either the number of synaptic vesicles in presynaptic terminals or in the number of membrane-attached synaptic vesicles in Complexin-1/2/3 TKO synapses. These findings correlate well with electrophysiological data for neurons lacking all three Complexin isoforms, which showed no changes in the size of RRP measured after the application of hyperosmolaric sucrose solution (Reim et al., 2001; Xue et al., 2007, 2008). It was possible to reveal a slight increase in the membrane-proximal vesicles in Complexin-1/2/3 TKO neurons. One possible explanation could be that neurons devoid of all Complexin isoforms have an impaired ability to release synaptic vesicles in response to action potential stimuli, leading to an accumulation of synaptic vesicles close to the active zone membrane. However, my data does not support the hypothesis of a clamping function for Complexins in mouse neurons, since in such a scenario I would have expected to see rather a slight decrease in the number of docked vesicles at the membrane, as had been shown in $C$. elegans Complexin mutants (Hobson et al., 2011). Taken together, the results of the present study support the hypothesis that Complexins are facilitators of synaptic vesicle release, which act on the assembled SNARE-complex after synaptic vesicle docking/priming. 

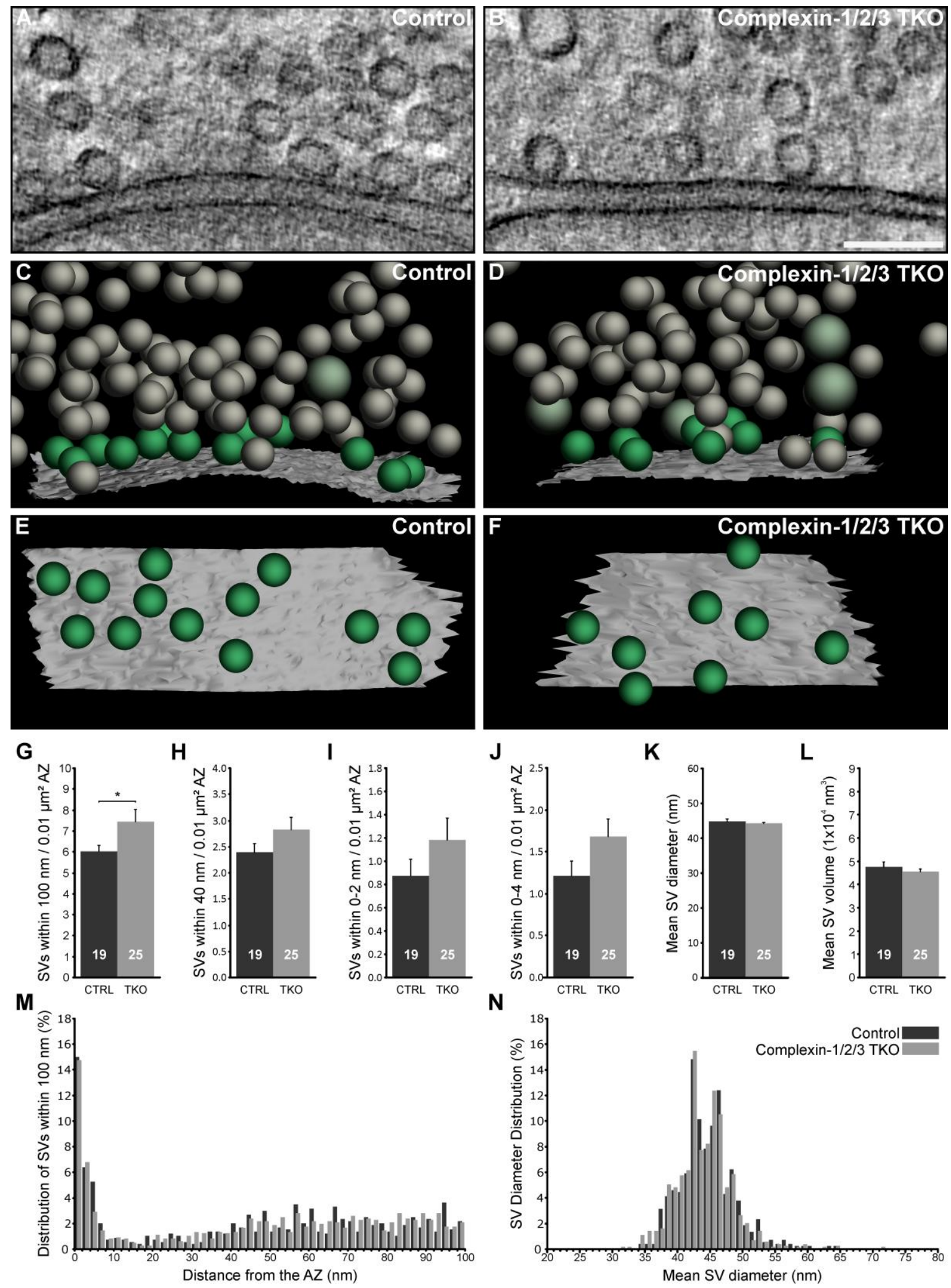

Figure 3.17. Three-dimensional electron tomographic analysis of synaptic vesicle docking in Complexin-1/2/3 TKO neurons

Tomographically reconstructed subvolumes of control (A) and Complexin-1/2/3 TKO (B) synapses from $200 \mathrm{~nm}$-thick sections. Three-dimensional models of tomographically reconstructed control (C) and Complexin-1/2/3 TKO (D) synaptic profiles. Ultrastructural features reconstructed in the models include the active zone plasma membrane (white), synaptic vesicles (membrane-attached, green; 
non-attached, grey) and endosomes (light blue). E, F Orthogonal views of control (E) and Complexin-1/2/3 TKO $(\mathbf{F})$ tomographic models displaying the spatial arrangement of membraneattached synaptic vesicles within the reconstructed active zone area. Mean number of SVs within $100 \mathrm{~nm}$ of the $A Z$ normalized to $A Z$ area $(\mathbf{G})$. Mean number of SVs within $40 \mathrm{~nm}$ of the AZ normalized to $A Z$ area $(\mathbf{H})$. Mean number of membrane-attached SVs (within 0-2 $\mathrm{nm}$ of the $A Z$ ) normalized to $A Z$ area (I). Mean number of membrane-attached and closely-tethered SVs (within 0$4 \mathrm{~nm}$ of the $\mathrm{AZ}$ ) normalized to $\mathrm{AZ}$ area $(\mathbf{J})$. Mean outer SV diameter (K). Mean SV volume including the membrane bilayer (L). Spatial distribution of SVs within a $100 \mathrm{~nm}$ distance of the $A Z$ membrane (M). Distribution of synaptic vesicle diameters of vesicles within $100 \mathrm{~nm}$ of the $A Z(\mathbf{N})$. G-L: Control: $\mathrm{N}=3, \mathrm{n}=19$; Complexin-1/2/3 TKO: $\mathrm{N}=3, \mathrm{n}=25$ (Mean + SEM), $\mathrm{P}<0.001$ : ${ }^{* \star *} ; \mathrm{P}<0.01$ : ${ }^{* *}$; $\mathrm{P}<0.05$ : *. M, N: (617 SVs in control and $953 \mathrm{SVs}$ in Complexin-1/2/3 TKO synaptic profiles). Scale bar: $\mathbf{B}, 100 \mathrm{~nm}$.

\subsubsection{The impact of the synaptic vesicle size on membrane-attachment}

To exclude the possibility that the severe docking deficits and the accumulation of membrane proximal synaptic vesicles observed in Munc13-1/2 DKO, SNAP25 KO and Synaptobrevin-2 KO synapses were an indirect consequence of the measured increases in synaptic vesicle size, I decided to reanalyze the size of exclusively membrane-attached (0-2 nm) and closely-tethered (0-4 nm) synaptic vesicles in these genotypes (Fig. 3.18).

When all synaptic vesicles within $100 \mathrm{~nm}$ of the active zone were included in the analysis, Munc13-1/2 DKO synapses exhibited an $8 \%$ increase in the synaptic vesicle diameter (control: $46.25 \pm 0.485, \mathrm{n}=16$; Munc13-1/2 DKO: $50.15 \pm 0.715, \mathrm{n}=15 ; \mathrm{P}<0.001$, ${ }^{* \star *}$; Fig. $3.4 \mathrm{~K}$ ) causing a $28 \%$ increase in the synaptic vesicle volume. In Munc13-1/2 DKO synapses, membrane-attached (0-2 nm; control: $46.14 \pm$ 0.453, $\mathrm{n}=76$; Munc13-1/2 DKO: $54.97 \pm 3.15, \mathrm{n}=3 ; \mathrm{P}=0.0106$, * Fig. $3.18 \mathrm{~A})$ and closely-tethered (0-4 nm; control: $46.10 \pm$ 0.366, $\mathrm{n}=129$; Munc13-1/2 DKO: $50.59 \pm 1.510, \mathrm{n}=10$; $\mathrm{P}=0.0014$, **; Fig. 3.18 B) synaptic vesicles exhibited similarly increased synaptic vesicle sizes. Analysis of synaptic vesicles within $100 \mathrm{~nm}$ of the active zone revealed a 14\% increase in the mean outer vesicle diameter for SNAP25 KO synapses (control: $46.12 \pm 0.482, \mathrm{n}=24$; SNAP25 KO: $52.53 \pm$ 0.586, $\mathrm{n}=25 / \mathrm{P}<0.001,{ }^{* *}$; Fig. $\left.3.8 \mathrm{~K}\right)$. With a focus on membrane-attached $(0-2 \mathrm{~nm}$; control: $44.86 \pm 0.336, \mathrm{n}=149$; SNAP25 KO: $\left.51.83 \pm 0.586, \mathrm{n}=32 / \mathrm{P}<0.001{ }^{* * *}\right)($ Fig. 3.18 C) and closely-tethered (0-4 nm; control: $45.03 \pm 0.279, \mathrm{n}=24$; SNAP25 KO: $52.08 \pm$ 0.665, $\mathrm{n}=25 / \mathrm{P}<0.001,{ }^{\star \star *}$ ) (Fig. $\left.3.18 \mathrm{D}\right)$ synaptic vesicles in SNAP25 KO synaptic profiles, a similar increase in the mean outer synaptic vesicle diameter was observed. Moreover, Synaptobrevin-2 synapses exhibited a 7\% increase in the synaptic vesicle diameter measured for all vesicles within $100 \mathrm{~nm}$ of the active zone (control: $45.81 \pm$ 0.450, n=25; Synaptobrevin-2 KO: $49.07 \pm 0.411, \mathrm{n}=24$ / $\mathrm{P}<0.001,{ }^{* *}$; Fig. $\left.3.13 \mathrm{E}\right)$. Membrane-attached (0-2 nm; control: $45.17 \pm 0.353, \mathrm{n}=149$; Synaptobrevin-2 KO: $48.03 \pm$ 
0.659, $\mathrm{n}=45$ / $\mathrm{P}<0.001,{ }^{* *}$; Fig. $\left.3.18 \mathrm{E}\right)$ and closely-tethered $(0-4 \mathrm{~nm}$; control: $45.23 \pm$ 0.301, $\mathrm{n}=209$; Synaptobrevin-2 KO: $47.91 \pm 0.522, \mathrm{n}=79$ / $\mathrm{P}<0.001,{ }^{* * *}$; Fig. $3.18 \mathrm{~F}$ ) showed comparable increases in synaptic vesicle diameter.

In summary, in all analyzed groups, synaptic vesicle membrane-attachment was still possible when vesicles exhibited an enlarged volume. These findings indicate that the increased synaptic vesicle size is unlikely to be responsible for the severe docking-deficits observed in Munc13-1/2 DKO, SNAP25 KO and Synaptobrevin-2 KO neurons.

A

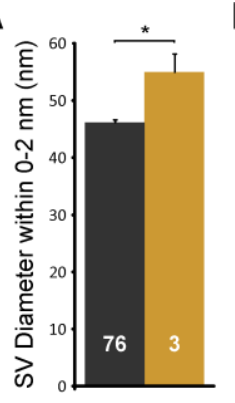

B

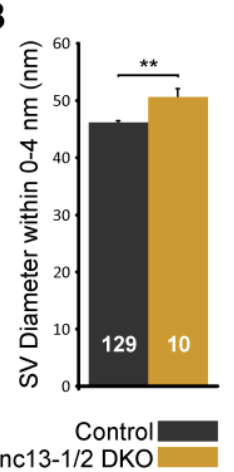

C

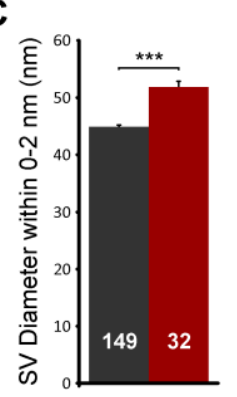

D

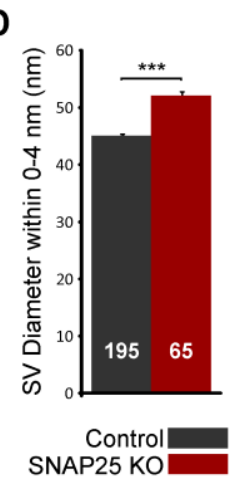

E

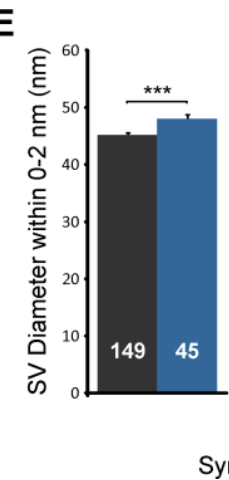

$\mathbf{F}$

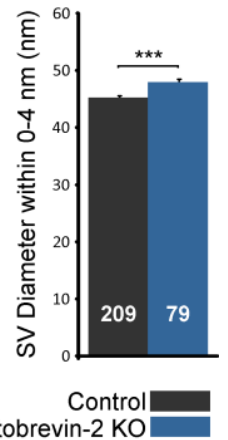

Figure 3.18. Enlarged vesicle diameters do not impair synaptic vesicle docking

A, B Mean outer SV diameter for membrane attached synaptic vesicles $(0-2 \mathrm{~nm}$; A) and vesicles within 0-4 nm (B) in Control and Munc13-1/2 DKO electron tomograms. Mean outer SV diameter for membrane attached synaptic vesicles (0-2 nm; C) and vesicles within 0-4 nm (D) in Control and SNAP25 KO electron tomograms. Mean outer SV diameter for membrane attached synaptic vesicles (0-2 nm; E) and vesicles within 0-4 nm (F) in Control and Synaptobrevin-2 KO electron tomograms. $n=$ number of membrane-attached vesicles (Mean $+\mathrm{SEM}$ ), $\mathrm{P}<0.001$ : ${ }^{* * *}, \mathrm{P}<0.01$ : ${ }^{* *}$; $\mathrm{P}<0.05:{ }^{*}$.

\subsubsection{SNARE mutants accumulate LDCVs in presynaptic terminals}

All mouse mutants analyzed in the course of this study have also been shown to function in the release of LDCVs from mouse chromaffin cells of the adrenal medulla (Ashery et al., 2000; Borisovska et al., 2005; Cai et al., 2008; Liu et al., 2008; Speidel et al., 2005; de Wit et al., 2006, 2009). Especially SNAP25, Syntaxin-1 and Synaptotagmin-1 have been implicated in LDCV docking in chromaffin cells, whereas the loss of CAPS in Drosophila has been shown to cause an accumulation of LDCVs at the neuromuscular junction (Renden et al., 2001; de Wit et al., 2006, 2009). We therefore decided to quantify the number of LDCVs in presynaptic terminals, which is inherently very low. 


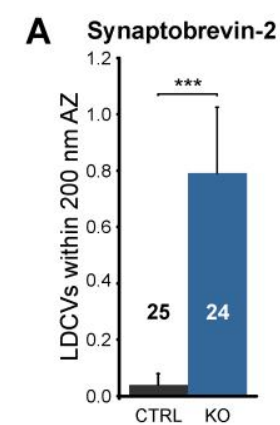

D
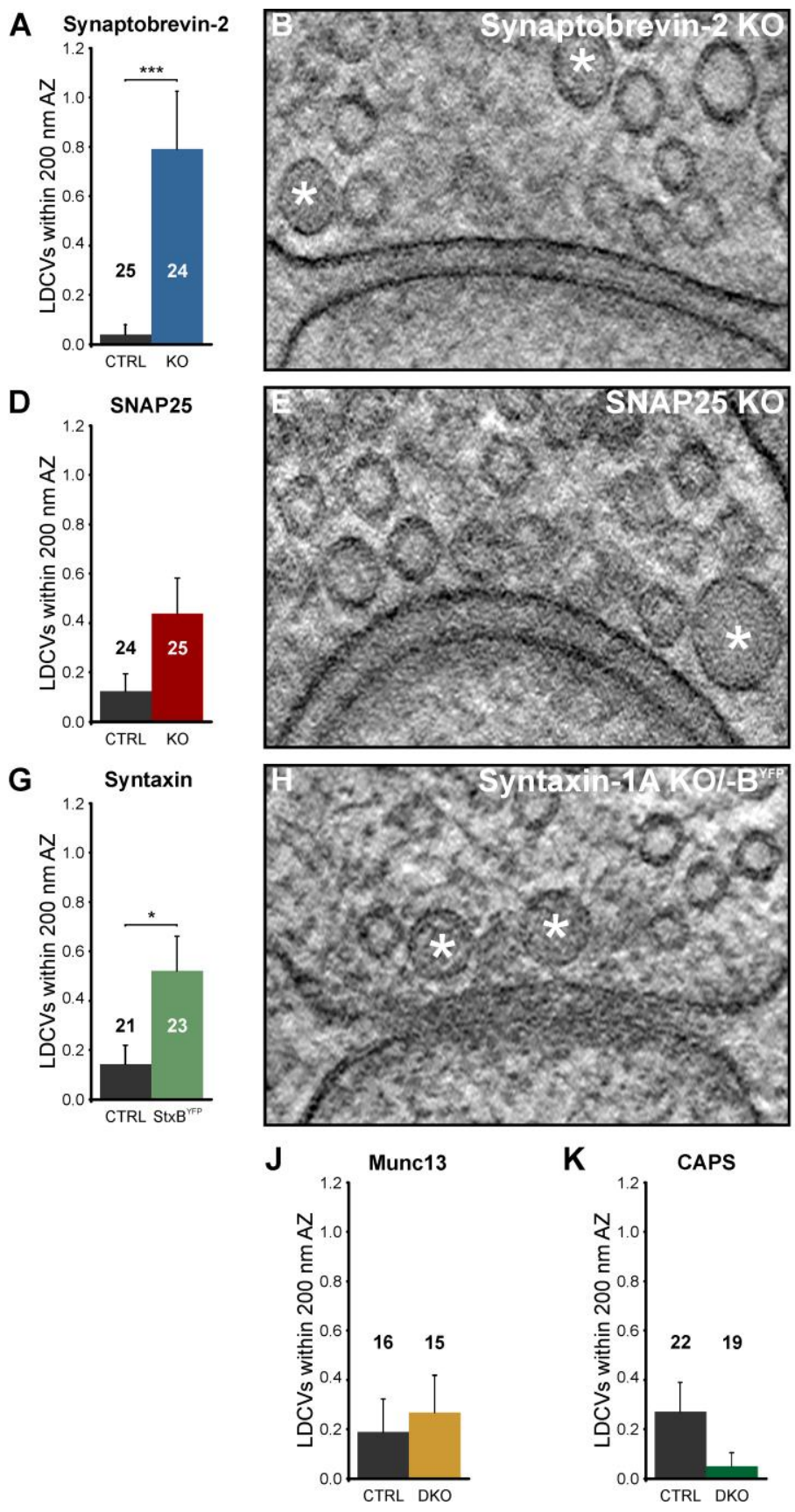
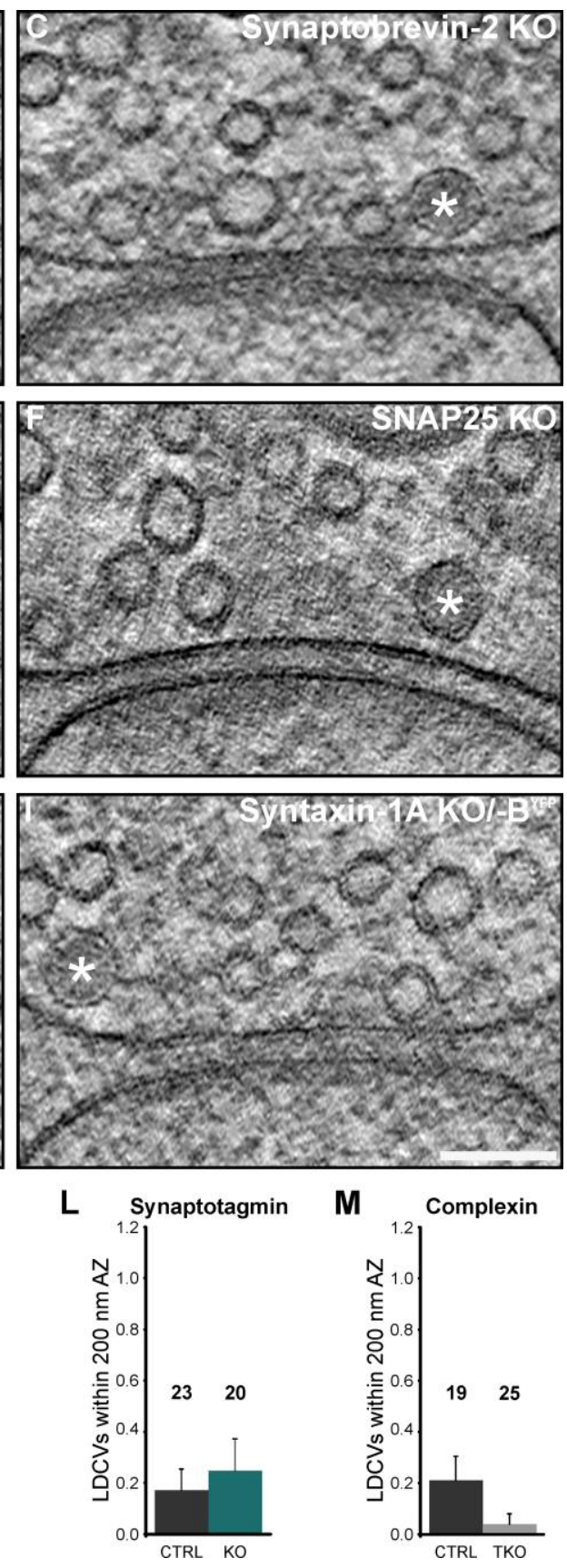

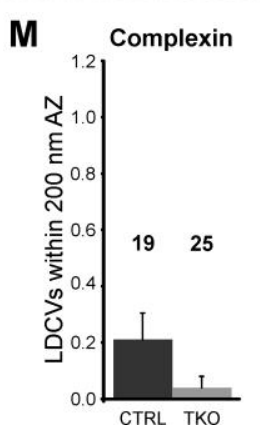

Figure 3.19. SNARE mutants accumulate LDCVs in presynaptic terminals

Mean number of large dense core vesicles (LDCVs) within $200 \mathrm{~nm}$ of the AZ per synaptic profile in Synaptobrevin-2 KO (A), SNAP25 KO (D), Syntaxin-1A KO / 1B ${ }^{\text {YFP }}(\mathbf{G})$, Munc13-1/2 DKO (J), CAPS-1/2 DKO (K), Synaptotagmin-1 KO (L) and Complexin-1/2/3 TKO (M) tomograms in comparison to their respective control groups. In Synaptobrevin-2 KO (B, C), SNAP25 KO (E, F) and Synatxin-1A 1B ${ }^{\text {YFP }}(\mathbf{H}, \mathbf{I})$ synaptic profiles, LDCVs (asterisk) have the tendency to accumulate within $40 \mathrm{~nm}$, but not to dock to the active zone. $n=$ number of tomograms (Mean + SEM), $\mathrm{P}<0.001:^{* * *}, \mathrm{P}<0.01:^{* *} ; \mathrm{P}<0.05:{ }^{*}$. Scale Bar in I: $100 \mathrm{~nm}$.

As previously mentioned, in two-dimensional electron microscopic analyses, I observed a slight, though not statistically significant, increase in the number of LDCVs per synaptic 
terminal in SNAP25 KO synaptic profiles (See 3.1.3.3.1; Fig. 3.7 $\mathrm{H}$ ). The number of LDCVs doubled in Syntaxin-1A/ -B ${ }^{\text {YFP }}$ synaptic profiles (See 3.1.3.4.1; Fig. 3.9 H) and the most dramatic increase in the number of LDCVs was observed in synaptic profiles from Synaptobrevin-2 KO slices (See 3.1.3.5.1; Fig. 3.11 H).

These increases in LDCV density in the presynaptic terminals of SNARE mouse mutants were confirmed by three-dimensional analysis of synaptic profiles by electron tomography (Fig.3.19). Since I did not image full synaptic terminals but rather focused on the active zone area, I limited the analysis to a $200 \mathrm{~nm}$ distance from the active zone. In synaptic profiles of Synaptobrevin-2 KO organotypic slices, the number of LDCVs within $200 \mathrm{~nm}$ was increased 19-fold in comparison to control tomograms (control: $0.040 \pm 0.040$, $\mathrm{n}=25$; Synaptobrevin-2 KO: $0.792 \pm 0.233, \mathrm{n}=24$ / $\mathrm{P}<0.001,{ }^{* * *}$; Fig. 3.19 A). Two Synaptobrevin-2 KO tomograms exhibited LDCVs within $40 \mathrm{~nm}$ of the active zone (Fig. $3.19 \mathrm{~B}, \mathrm{C})$. These vesicles were in close proximity to the plasma-membrane, but not docked. In SNAP25 KO synaptic profiles, the number of LDCVs within $200 \mathrm{~nm}$ of the active zone membrane increased 3.5-fold (control: $0.125 \pm 0.069, \mathrm{n}=24$; SNAP25 KO: $0.440 \pm 0.142, n=25$ / $P=0.0858$, n.s.; Fig. 3.19 D). In four SNAP25 KO synaptic profiles, LDCVs were detected within $40 \mathrm{~nm}$ of the active zone, but were not in contact with the plasma membrane (Fig. 3.19 E, F). Synaptic profiles from Syntaxin-1A/ -B ${ }^{\text {YFP }}$ neurons revealed a 3.6-fold increase in the number of LDCVs within $200 \mathrm{~nm}$ of the active zone (control: $0.143 \pm 0.078, n=21$; Syntaxin-1A/ $-B^{Y F P}: 0.522 \pm 0.139, n=23 / P=0.0314$, *; Fig. $3.19 \mathrm{G})$. Three Syntaxin-1A/ -B ${ }^{\text {YFP }}$ synaptic profiles contained LDCVs within $40 \mathrm{~nm}$ of the active zone that were not membrane-attached (Fig. 3.19 H, I). No LDCVs were detected within $40 \mathrm{~nm}$ of the active zone membrane in control groups for the SNARE mutants.

In Munc13-1/2 DKO (control: $0.188 \pm 0.136, \mathrm{n}=16$; Munc13-1/2 DKO: $0.267 \pm 0.153, \mathrm{n}=15$ / $P=0.7012$, n.s.; Fig. 3.19 D), CAPS-1/2 DKO (control: $0.273 \pm 0.117, n=22$; CAPS-1/2 DKO: $0.053 \pm 0.053, n=19 / P=0.1126$, n.s.; Fig 3.19 E), Synaptotagmin-1 KO (control: $0.174 \pm 0.081, \mathrm{n}=23$; Synaptotagmin-1 KO: $0.250 \pm 0.123, \mathrm{n}=20 / \mathrm{P}=0.5993$, n.s.) and Complexin-1/2/3 TKO (control: $0.211 \pm 0.096, n=19$; Complexin-1/2/3 TKO: $0.040 \pm 0.040$, $\mathrm{n}=25 / \mathrm{P}=0.0808$, n.s.) the number of LDCVs within $200 \mathrm{~nm}$ of the active zone remained unchanged between controls and mutant synaptic profiles. However, despite the absence of LDCV accumulation within profiles of Synaptotagmin-1 KO synapses, three of the acquired tomograms contained LDCVs within $40 \mathrm{~nm}$ of the active zone that were close to, but not in physical contact with the plasma membrane (examples not shown). In Munc131/2 DKO, CAPS-1/2 DKO and Complexin-1/2/3 TKO synaptic profiles, no LDCVs were found within $40 \mathrm{~nm}$ of the active zone. However, in the Complexin-1/2/3, CAPS-1/2 and Synaptotagmin-1 control groups, one synaptic profile each contained a single LDCV within 
$40 \mathrm{~nm}$ of the active zone, but LDCV docking at the active zone membrane was never observed.

3.2.

\section{Generation and characterization of a Munc13-1 conditional knock-out (CKO) mouse line}

The Munc13 family of priming proteins is essential for synaptic transmission. Munc13-1 is the most abundant isoform in the central nervous system and the loss of Munc13-1 courses perinatal lethality and severe deficits in glutamatergic transmission (Augustin et al., 1999a). Munc13-2- and Munc13-3-deficient mice are viable and fertile and neurons function normally under basal conditions, indicating a dominant role for Munc13-1 in mammalian synapses. Over the last years an important role of Munc13s in regulating synaptic plasticity became evident (Breustedt et al., 2010; Chen et al., 2013; Lipstein et al., 2013; Yang and Calakos, 2011; Zhao et al., 2012a, 2012b). However, due to the lethal phenotype, a detailed study of the function of Munc13-1 in the mature, developed brain and therefore in a physiological network, has not yet been possible. I therefore set out to generate a conditional Munc13-1 mouse line, where Munc13-1 expression can be shut down at different developmental time-points or in specific subsets of neurons.

\subsubsection{Generation of a CKO targeting vector}

The aim was to generate a mouse in which exon 21 in the Munc13-1 gene is flanked by two loxP sites, so that upon site-specific Cre-recombinase activity in the neurons exon 21 will be deleted. This, in turn, is predicted to result in a frame shift in the gene transcription product, and therefore to a loss of the Munc13-1 protein.

For the generation of a conditional knockout (CKO) targeting vector, I chose recombination-mediated genetic engineering (recombineering), a method based on homologous recombination via gap repair in E.coli bacterial cells. This method utilizes the specialized E.coli strains E350 and SW106 (Lee et al., 2001; Liu et al., 2003; Warming et al., 2005), derived from DH10B BAC DNA host cells and modified to express enzymes required for gap repair (Exo, Beta, and Gam) in a temperature-dependent manner from a defective prophage $\lambda$ (Lee et al., 2001). In addition to the heat-controlled expression of Exo, Beta, and Gam, E350 and SW106 cells encode the adenoviral P1 bacteriophage 
cyclization recombination (Cre)-recombinase under an L-arabinose-inducible promoter. E350 and SW106 strains only differed by an additional gaK-based selection system integrated into SW106, which I did not employ in this study (Warming et al., 2005). The expression of these enzymes enables recombination of DNA fragments between vectors carrying homologous sequences.

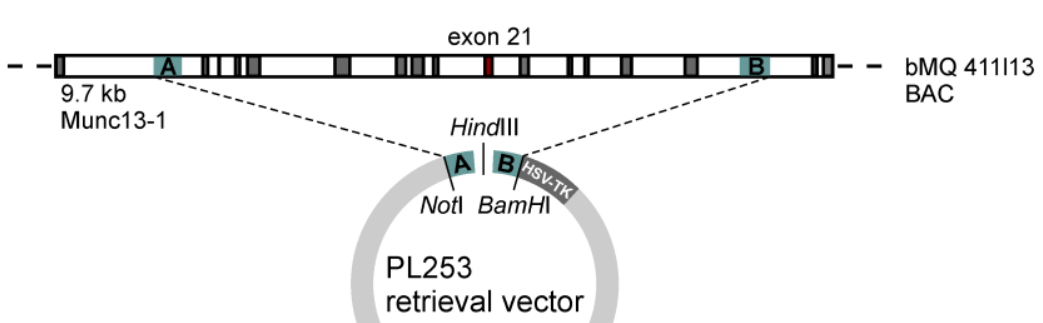

\section{BAC DNA retrieval via gap repair}

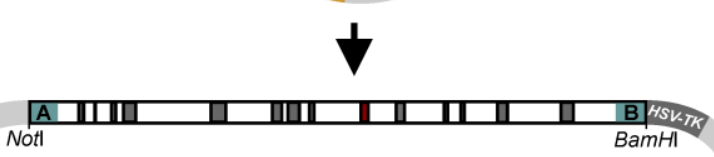

2. Targeting of the $1^{\text {st }}$ loxP site
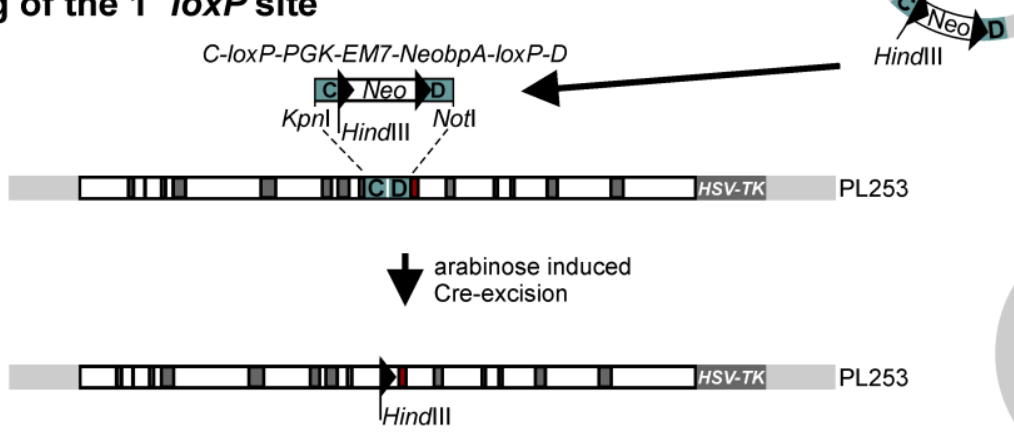

3. Targeting of the $2^{\text {nd }} l o x P$ site

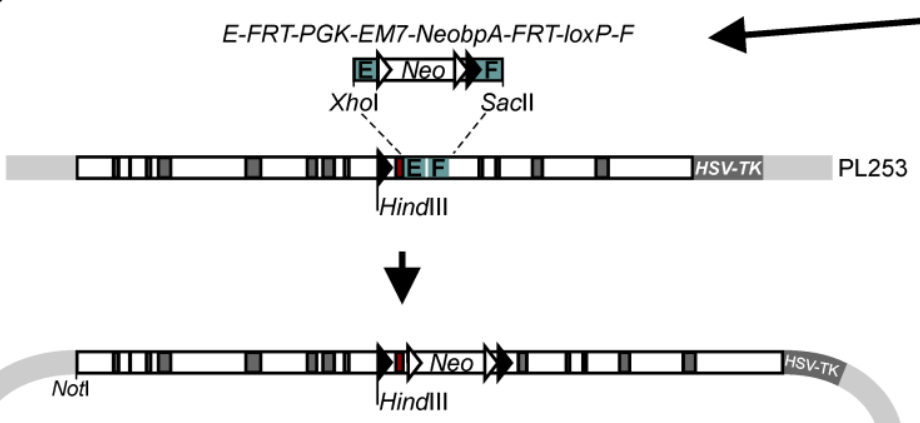

Munc13-1 cko targeting vector 
Figure 3.20. Generation of a Munc13-1 conditional knock-out targeting vector

Schematic representation of the cloning strategy for a Munc13-1 conditional knock-out (cko) targeting vector. Exons are labeled as grey boxes, introns as white boxes and exon 21 as a red box. White triangles indicate loxP sites, black triangles indicate FRT sites. 1. BAC DNA retrieval via gap repair: Two homology arms $(A+B)$ were amplified by PCR and subcloned into the PL253 retrieval vector. The vector was linearized by Hindll digest. A $9.7 \mathrm{~kb}$ fragment of the Munc13-1 gene containing exon 21 was retrieved from the SV129 embryonic stem (ES) cell derived bacterial artificial chromosome (BAC) plasmid bMQ411/13 via homologous recombination into the PL253 retrieval vector. 2. Targeting of the first loxP site: The two homology arms $(\mathrm{C}+\mathrm{D})$ were amplified by PCR and subcloned into the PL452 vector plasmid to flank a floxed neomycin resistance gene (Neo) cassette. The floxed Neo cassette with the two homology arms was excised by Kpnl and Notl digestion, together with Scal to cut the plasmid backbone in the Amp gene. The floxed Neo cassette was inserted upstream of exon 21 via homologous recombination and excised after Larabinose-induced Cre-recombinase expression, resulting in a single loxP site with a diagnostic Hindlll restriction site. 3. Targeting of the second loxP site: The two homology arms $(E+F)$ were amplified by PCR and subcloned into the PL451 vector plasmid to flank a Neo cassette flanked by FRT sites and a downstream loxP site. The FRT site flanked Neo cassette with the two homology arms was excised by Xhol and Sacll digest, together with Scal to cut the plasmid backbone in the Amp gene, and inserted downstream of exon 21 via homologous recombination. The Neo cassette, $H S V-T K$ cassette, and FRT and loxP sites are not drawn to scale. Neo, neomycin resistance gene; HSV-TK, herpes simplex virus thymidine kinase; Amp, Ampicilin. Based on (Liu et al., 2003)

Using recombineering, I retrieved a $9.7 \mathrm{~kb}$ Munc13-1 gene fragment containing exons 1326. This fragment was retrieved from a BAC, namely the 129SV ES cell derived BAC clone bMQ 441113 containing the full Munc13-1 genomic sequence. bMQ 441113 is part of a 129SV ES cell DNA BAC library and contains a pBACe3.6 vector backbone with a chloramphenicol resistance gene for selection (Adams et al., 2005; Frengen et al., 1999). The fragment was retrieved into a plasmid containing a $\mathrm{MC1}$ promotor controlled herpes simplex virus thymidine kinase cassette (MC1TK). I subsequently introduced loxP sites and a neomycin resistance (Neo) cassette for positive selection in embryonic stem (ES) cells (Liu et al. 2003). The individual recombineering steps for the generation of the targeting vector are presented schematically in Fig. 3.20, and the strategy for the generation of the Munc13-1 CKO mouse line is illustrated in Fig.3.21 A.

\subsubsection{Retrieving of BAC DNA fragment into the PL253 vector}

In the first step, a $9.7 \mathrm{~kb}$ fragment containing the targeted exon 21 and the flanking longand short arm for homologous recombination in ES cells was retrieved via recombineering from the BAC into the pBluescript-based targeting vector backbone PL253, containing a modified MC1TK cassette (Liu et al. 2003) (Fig. 3.20). First, the BAC clone bMQ 441/13 DNA was amplified and a restriction analysis was performed to confirm the identity of the clone. $4 \mu \mathrm{g}$ freshly prepared BAC was electroporated into electrocompetent EL350 cells 
and the bacteria were plated on LB agar plates containing chloramphenicol to select for the presence of the BAC plasmid. Colonies were chosen and BAC DNA was purified. 10 $\mu \mathrm{l}$ of purified BAC DNA before and after transformation was digested overnight at $37^{\circ} \mathrm{C}$ with 20 units of the restriction enzymes Sall, Nrul and Clal in $50 \mu l$ volumes each. The respective restriction patterns were compared after pulsed field gel electrophoresis on an agarose gel. The expected band pattern for all restriction enzymes was found in all clones tested, with the exception of $\mathrm{Nrul}$, which did not cut at one predicted restriction site (data not shown). This finding indicated small differences between the C57BL/6J reference sequence and the SV129 ES cell DNA. Indeed, during the generation of the Munc13-1 CKO targeting vector, some minor differences to the C57BL/6J reference sequence were observed after sequencing. However, these differences in the nucleotide sequence did not alter the identity of the encoded amino acids and were therefore considered to reflect genomic polymorphisms between strains.

In the next step, short homology arms (labeled A and B; Fig 3.20.1) were amplified from the BAC clone using PCR and subcloned into PL253, resulting in the plasmid PL253-AB (see 2.2.2.16.1.). These short homology arms define the borders of the desired sequence to be retrieved from the BAC clone. For the retrieval of the $9.7 \mathrm{~kb} B A C$ fragment into the PL253 retrieval vector, $600 \mathrm{ng}$ of the PL253-AB DNA was linearized by 20 units Hindll for 2 h. Next, 170 ng DNA was electroporated into electrocompetent EL350 cells that contain the $\mathrm{bMQ} 441113 \mathrm{BAC}$ and in which the expression of the gap repair enzymes was induced. Amp ${ }^{r}$ colonies (i.e. colonies transformed with PL253-AB) were screened by PCR for the integration of the 9.7 kb Munc13-1 genomic sequence into the PL253-AB retrieval vector. In three independent experiments, 120 colonies were screened but gave rise to only one positive clone. To select against plasmid multimers, which can occur in pBluescript-based vectors by rolling circle replication during the gap repair process, $1 \mathrm{ng}$ of the gap-repaired plasmid was retransformed into Electro10 Blue cells and Ampr colonies were selected and tested for the presence of the targeted plasmid.

\subsubsection{Targeting of the first loxP site upstream of Munc13-1 exon 21}

In order to introduce one loxP site upstream exon 21 of the Munc13-1 gene, a Neo cassette flanked by two loxP sites was first introduced into the Munc13-1 genomic sequence via site-specific recombineering (Fig. 3.20.2). In a subsequent step, the Neo cassette was excised by Cre-mediated recombination after L-arabinose induced Crerecombinase expression in SW106 E.coli cells. The Neo resistance gene that was used 
(from the PL452 vector) is flanked by two loxP sites and is regulated by two promotors: the phosphoglycerate kinase I (PGK) promotor, which permits the expression of the Neo resistance gene in eukaryotic cells, and the EM7 promotor, which enables prokaryotic cell expression. Moreover, the Neo cassette is followed by a bovine growth hormone polyadenylation site (bpA), a termination signal for protein expression in eukaryotic cells.

Short homology arms (Labeled C and D; Fig. 3.20.2) were PCR amplified from the BAC DNA and subcloned into PL452 (See 2.2.2.16.2.). The Neo cassette flanked by the two short homology arms was cut out of the resulting PL452 vector by digestion with 20 units of Kpnl and Notl as well as Scal (to cut the plasmid backbone in the ampicilin resistance gene). $100 \mathrm{ng}$ of the C-IoxP-PGK-EM7-NeobpA-IOXP-D fragment were electroporated together with $10 \mathrm{ng}$ of the PL253 vector containing the $9.7 \mathrm{~kb}$ Munc13-1 genomic insert into electrocompetent and induced SW106 cells and Amp ${ }^{r}$ (PL253 vector) and $\mathrm{Kan}^{r}$ (Neo cassette) colonies were selected. Five independent experiments were performed, in total $\sim 450$ clones were screened, however only the last attempt gave rise to 11 positive colonies. Positive colonies contained a mix of gap-repaired and non-gap-repaired plasmids. Therefore, $1 \mathrm{ng}$ of the gap-repaired plasmid was retransformed into XL1-Blue cells and colonies resistant to $\mathrm{Amp}^{r}$ and $\mathrm{Kan}^{\mathrm{r}}$ were selected. $1 \mathrm{ng}$ of the retrieval vector containing the IoxP-PGK-EM7-NeobpA-IoxP cassette was then electroporated into electrocompetent SW106 cells after arabinose-induced Cre-expression. Cre activity resulted in the removal of the Neo cassette by Cre-mediated recombination between the two loxP sites in all selected $\mathrm{Amp}^{r}$ colonies, leaving only one loxP site upstream of Munc13-1 exon 21.

\subsubsection{Targeting of the second loxP downstream of Munc13-1 exon 21}

In order to introduce the second loxP site and a Neo cassette for positive selection in ES cells, a Neo cassette, flanked by two flippase (FLP) recombinase target (FRT) sequences and followed by a single loxP site was integrated downstream of Munc13-1 exon 21 by site-specific recombination in E.coli cells. Generation and subcloning of short homology arms amplified from the BAC DNA (termed E and F: Fig. 3.20.3) into PL451 is described in 2.2.2.16.3. The expression of the Neo resistance gene is controlled by a PGK and an EM7 promotor together with a bpA site. The Neo cassette, flanked by the two short homology arms, was cut out of the E-FRT-PGK-EM7-NeobpA-FRT-loxP-F containing vector PL451 by subsequent digestion with 20 units of Xhol and Sacll as well as Scal to cut the plasmid backbone in the ampicilin resistance gene. $40 \mathrm{ng}$ of the E-FRT-PGK-EM7- 
NeobpA-FRT-loxP-F fragment were electroporated together with $10 \mathrm{ng}$ of the PL253 retrieval vector from the previous step into electrocompetent and induced cells. Amp ${ }^{r}$ (PL253 vector) and $\mathrm{Kan}^{r}$ (Neo cassette) colonies were selected. 32 colonies were screened and four positive clones were obtained, which contained a mixture of gaprepaired and non-gap-repaired plasmids. $1 \mathrm{ng}$ of the plasmid DNA was retransformed into XL1-Blue cells and $\mathrm{Amp}^{\mathrm{r}}$ and $\mathrm{Kan}^{\mathrm{r}}$ colonies were selected. Positive clones contained a single FRT-PGK-EM7-NeobpA-FRT-IoxP cassette downstream of the Munc13-1 exon 21 in addition to the one loxP site upstream of exon 21 (Fig. 3.23.3 and 3.24 A).

\subsubsection{Generation of recombinant ES cells and mice}

In order to prepare the Munc13-1 CKO targeting vector for ES cell electroporation, the plasmid was purified with the EndoFree Plasmid Maxi Kit (Qiagen). A total of $450 \mu \mathrm{g}$ DNA was liniarized in nine tubes, each one containing $50 \mu \mathrm{g}$ DNA and 100 units Notl in a volume of $300 \mu \mathrm{l}$ and in the presence of $1 \times$ BSA for 5 hours at $37^{\circ} \mathrm{C}$. To check the digestion efficiency, $1 \mu$ l of each tube was loaded onto a gel. The Notl linearized CKOtargeting vector was then electroporated into SV129/Ola ES cells and the transformed clones were selected for their G418 and ganciclovir resistance. G418, also called geneticin, is an antibiotic used for positive selection in eukaryotic cells expressing the Neo cassette. Ganiclovir is an anti-viral drug that becomes toxic upon phosphorylation by HSVTK and is therefore used for negative selection to prevent ES cell clones that had random insertion of the targeting vector backbone from growing. Out of 192 clones analyzed by Southern blot after Hindll digest and probed with a $\mathrm{P}^{32}$-labelled probe that is located upstream of the sequence used in the targeting vector (5' probe; Fig. 3.24A), 29 positive clones were identified. Because of an introduced Hindll site along with the $1^{\text {st }}$ loxP site, which was located upstream of exon 21 , homologous recombinants carrying the CKO allele generated a $9.8 \mathrm{~kb}$ (wt) band and a $8.4 \mathrm{~kb}$ (mutant) band after probing with the 5' probe (Fig. $3.21 \mathrm{~B}$ ). From these colonies, 12 clones were subsequently tested after Mfel digestion by hybridization with a $\mathrm{P}^{32}$-labelled probe located downstream of the sequence used in the targeting vector ( 3 ' probe), and with a $\mathrm{P}^{32}$-labelled probe targeting the Neo cassette sequence (Neo probe). In ES cells that carry the cko allele in the desired location, a $7.9 \mathrm{~kb}$ (WT) band and a $9.7 \mathrm{~kb}$ (mutant) band can be detected after probing with the 3' probe as the length of the FRT-PGK-EM7-NeobpA-FRT-loxP cassette had been added (Fig. $3.21 \mathrm{~B}$ ), and a single $9.6 \mathrm{~kb}$ band can be detected after probing with the Neo probe. No additional signal was detected in the Southern blot probed for Neo, which 
indicated that these clones exhibited no random integration of the targeting vector into the genome. All 12 clones were tested positive for 5', 3' and a Neo probe (data not shown). The Munc13-1 allele containing the Neo cassette was termed Munc13-1 Neo.

The ES cell clones A4, B7 and H6 were chosen for blastocyst injection. Blastocyst injection was conducted by Monica Schindler in the Transgenic Animal Facility of the MaxPlanck-Institute of Experimental Medicine, Göttingen, Germany. The blastocysts for the procedure were derived from $\mathrm{C} 57 \mathrm{BL} / 6 \mathrm{~N}$ and $\mathrm{C} 57 \mathrm{BL} / 6$ albino mice and planted into hormone-treated pseudo-pregnant C57BL/6 females. ES cells originating from the SV129/Ola line carry the agouti allele, which adds a yellow shimmer to mouse fur and leads to a brownish hair color in C57BL/6 and to a cream-colored appearance of C57BL/6 albino chimeras. A high percentage of brown or cream colored fur corresponded to a high degree of chimerism. From ES cell clone $\mathrm{H} 6$, seven male chimeras were identified by

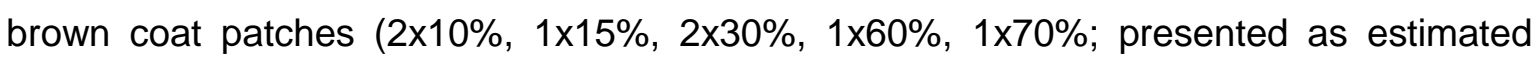
percentage of the coat surface), Of which two males were germline competent $(60 \%$ and $70 \%$ ). From ES clone B7 two male chimeras (1x30\% and $1 \times 40 \%)$ were obtained with one being germline competent (40\%). The chimeric offsprings were crossed with FLPeR ("flipper") mice, that express the FLP-recombinase, which enables, via site-specific FLPrecombination, the excision of the Neo cassette at the FRT sites flanking the Neo cassette, leaving a single FRT site and one loxP site (Farley et al., 2000). As the FLPeR allele was crossed into the $\mathrm{C} 57 \mathrm{BL} / 6 \mathrm{~N}$ genetic background, which encodes for black fur color, germ-line transmission of the Munc13-1 Flp allele in the SV129/Ola background would only produce brown-colored offspring, independent of the origin of the blastocysts (C57BL/6N or C57BL/6 albino). The two chimeric animals from ES cell clone $\mathrm{H} 6$ produced altogether eight brown-colored pups, the one chimeric animal from ES cell clone B7 produced five brown-colored pups. As the excision of the Neo cassette was detected only in a subset of cells after one crossing with the flipper mice, the animals were crossed with C57BL/6J mice and offspring exhibiting germ-line transmission of the Cre-recombined allele were selected. The Munc13-1 allele after Flp-mediated excision of Neo cassette was termed Munc13-1 Flp and was characterized by a single loxP site upstream and a FRT and loxP site downstream of exon 21 in the Munc13-1 gene locus. Offspring homozygous for the Munc13-1 Flp allele was viable and fertile. For all following experiments, I only worked with offspring generated from the ES cell clone $\mathrm{H} 6$. 
A

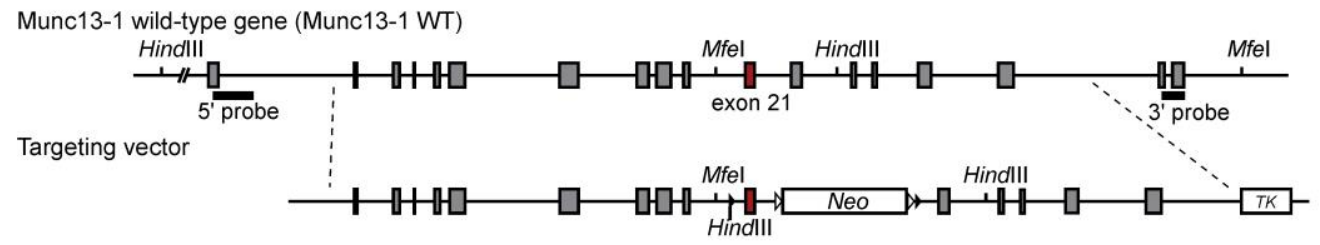

Targeted Munc13-1 gene (Munc13-1 Neo)

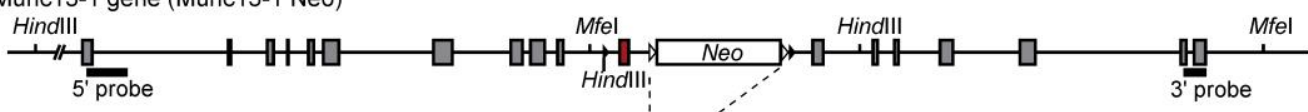

Munc13-1 gene after FLP-mediated recombination (Munc13-1 Flp)

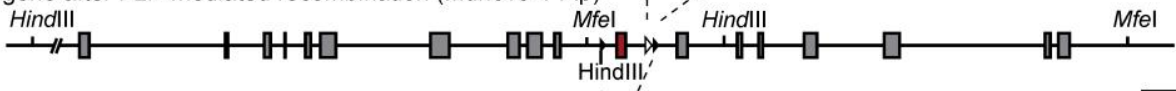

Munc13-1 gene after Cre-mediated recombination (Munc13-1 Cre) $)^{\prime}$,

$$
\text { HindIII }
$$

Mfel HindlI

(a)

11-

C

B

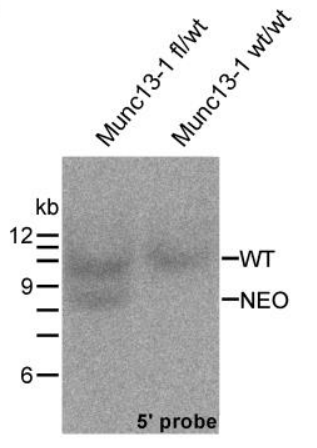

D

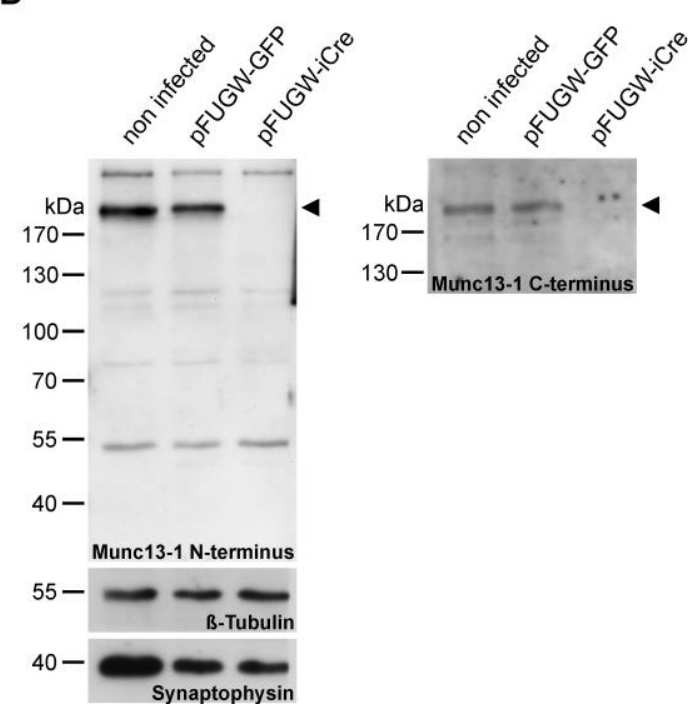

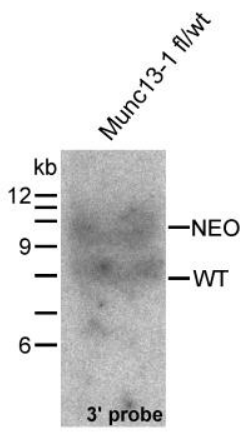

E
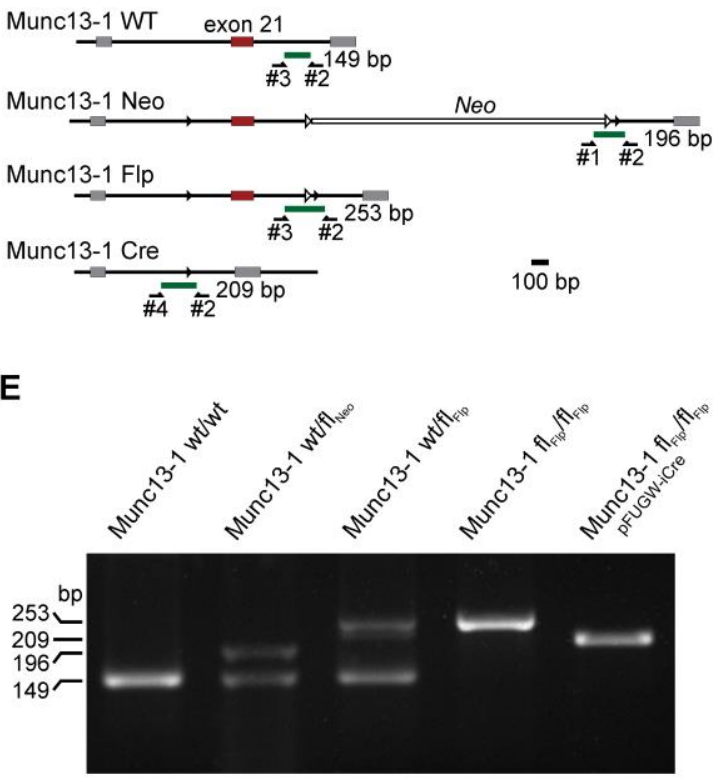

\section{Figure 3.21. Generation and characterization of Munc13-1 conditional knock-out mice}

A Schematic representation of the Munc13-1 wild-type gene (Munc13-1 WT), the targeting vector, the mutated gene after homologous recombination (Munc13-1 Neo), the mutated gene after flippase (FLP)-mediated excision of the neomycin ( $\mathrm{Neo}$ ) resistance cassette (Munc13-1 Flp), and the mutated gene after adenoviral cyclization recombination (Cre)-mediated recombination (Munc13-1 Cre). The targeted exon 21 is indicated in red, remaining exons are labeled grey. White triangles indicate loxP sites, black triangles indicate FRT sites. The 5' and 3' probes, represented by black horizontal bars, were used for Southern blot analysis. Hindll and Mfel restriction sites employed for diagnostic digestion for Southern blot analysis are indicated. TK: herpes simplex virus 
thymidine kinase gene B $5^{\prime}$ probe Southern blot hybridization after Hindlll-digestion of embryonic stem (ES) cell clone DNA produced a $9.8 \mathrm{~kb}(\mathrm{wt})$ band and an $8.4 \mathrm{~kb}(\mathrm{Neo})$ band. 3' probe Southern blot hybridization after Mfel-digestion of ES cell clone DNA produced a $7.9 \mathrm{~kb}(\mathrm{wt})$ band and a $9.7 \mathrm{~kb}(\mathrm{Neo})$ band. C Schematic representation of the genotyping strategy for the Munc13-1 WT, Munc13-1 Neo, Munc13-1 Flp and Munc13-1 Cre allele. The positions of the diagnostic polymerase chain reaction (PCR) fragments are indicated in green together with their expected sizes in base pairs (bp). The primers (\#1-\#4) used for the respective PCR reactions are not drawn to scale. D Western blot analysis of Munc13-1 levels ( $200 \mathrm{kDa}$, black arrowheads) as well as BTubulin and Synaptophysin loading control levels in cell homogenates from a Munc13-1 Flp hippocampal neuron mass culture that was either not infected (lane 1) or infected on DIV 2 with pFUGW-GFP- (lane 2) or pFUGW-iCre- (lane 3) containing lentiviral particles. Absence of Munc131 in pFUGW-iCre infected neurons was confirmed with an antibody directed against the $\mathrm{N}$-terminal part of Munc13-1 (\#40) (upper left panel) (Varoqueaux et al. 2005; Cooper et al. 2012) and with an antibody against the C-terminal part of Munc13-1 (\#N395AP) (right panel) (Betz et al., 1997). E Genotyping results for the indicated genotypes obtained from PCR amplification of either mouse tail DNA (Munc13-1 wt/wt, Munc13-1 wt/f $\left.\right|_{f|1|}$, Munc13-1 $\mathrm{fl}_{\mathrm{fip}} / \mathrm{f}_{\mathrm{ftp}}$ ), ES cell clone DNA (Munc13-1 $w t /\left.f\right|_{N e o}$ ) or DNA lysates from pFUGW-iCre infected neuron cultures (Munc13-1 $\mathrm{f}_{\mathrm{fip}} / \mathrm{f}_{\mathrm{flp}}-\mathrm{pFUGW}$ iCre).

\subsubsection{Genotyping Strategy}

The primers used in the genotyping PCR reaction and the predicted PCR DNA products for the individual stages of the generation of the mouse line are depicted in Fig.3.21 C. Genotyping of mouse DNA prepared from tail tissue was used at all breeding stages and prior to all experiments to confirm the genotype. The respective DNA fragment patterns were visualized on an agarose gel, as presented in Fig. 3.21 E. Before the FLP-mediated recombination of the Neo cassette, the genotyping reaction contained three primers (\#1, \#2, \#3). Under the given PCR conditions (see materials and methods), the wild-type Munc13-1 allele was identified by a 149 bp long PCR fragment (amplified from the primer pair \#2 and \#3). The Munc13-1 Neo allele was detected by the presence of a $196 \mathrm{bp}$ long PCR product between primers \#1 and \#2, as primer \#1 anneals within the Neo cassette. Excision of the Neo cassette by Flp-mediated recombination (Munc13-1 Flp allele) produced a $253 \mathrm{bp}$ long PCR product using the primers \#2 and \#3. At this stage, we occasionally observed an additional PCR product of approximately $230 \mathrm{bp}$ (data not shown). The band only appeared when a PCR mix containing all primers was used, and not when a single primer pair was used, and was therefore considered to be non-specific. Primers \#2 and \#3 were used from that point on for routine genotyping of the mouse line.

In order to produce a full Munc13-1 KO allele, the Cre/loxP system was employed (Kaartinen and Nagy, 2001). Site-specific Cre-recombinase mediated recombination would lead to the deletion of exon 21 of Munc13-1 and therefore induce a predicted frame-shift mutation, which would result in a complete Munc13-1 KO allele, termed Munc13-1 Cre. The Munc13-1 Cre allele was predicted to produce a 209 bp long PCR 
product with the primers \#2 and \#4. To examine whether a full deletion can occur in neurons carrying the Munc13-1 Flp allele, I infected hippocampal neurons in vitro from Munc13-1 Flp mice with a lentivirus that carries the cre cassette (pFUGW-iCre; see section 3.2.4 for more details). DNA extracts from these cells were collected and analyzed with the genotyping PCR (Fig. 3.21 E lane 5). Indeed, a single band of roughly $209 \mathrm{bp}$ was observed, indicating efficient Cre-recombination. To show that Cre-mediated recombination is possible also in vivo, we started to cross the Munc13-1 Flp allele into the Ella-Cre mouse line that expresses the Cre-recombinase protein controlled by the adenoviral Ella promotor in cells from all body tissues (Lakso et al., 1996). The expression from the Ella promotor starts at early embryonic stages that thereby results in early excision of the Neo cassette, which is expected to produce the Munc13-1 Cre allele in all neurons. At this point we were able to obtain mice that were heterozygous for the Munc13-1 Cre allele and I can therefore confirm Cre-mediated deletion of Munc13-1 exon 21 in vivo (data not shown). In a PCR amplification using the primers \#2 and \#4, the wildtype Munc13-1 allele and the not Cre-recombined Munc13-1 Flp allele were expected to additionally produce $746 \mathrm{bp}$ and $953 \mathrm{bp}$ long fragments, respectively (bands not shown in Fig. 3.21 E.). These PCR products were of no diagnostic use to us.

\subsubsection{Characterization of the Munc13-1 CKO mouse line}

In order to analyze Munc13-1 protein expression after Cre-mediated deletion of exon 21, I employed lentiviral expression of the Cre-recombinase protein from the pFUGW-iCre vector plasmid in cultured hippocampal neurons from Munc13-1 Flp mice. Dissociated neuron cultures were infected at DIV 2 with lentiviral particles containing either the empty pFUGW plasmid that expresses GFP only (pFUGW-GFP) or the pFUGW-iCre plasmid that expresses Cre-recombinase protein and GFP. Western blot analysis was performed on homogenates produced at DIV 21 to detect the Munc13-1 protein levels. I tested for the presence of the full length Munc13-1 protein by using an antibody directed against a C-terminal epitope of Munc13-1 (\#N395AP) (Fig. 3.21 D right panel) (Betz et al., 1997). In addition, I used an antibody directed against an N-terminal epitope of Munc13-1 (\#40), which specifically detects Munc13-1 without detectable cross-reactivity with other Munc13 isoforms (Cooper et al., 2012; Varoqueaux et al., 2005). The results revealed a complete loss of the Munc13-1 protein in the homogenates infected with pFUGW-iCre compared with the non-infected or pFUGW-GFP infected cell homogenates (Fig. $3.21 \mathrm{D}$ ). I was not able to detect Munc13-1 full length protein as well as any residual $\mathrm{N}$-terminal fragment in 
cell homogenates from pFUGW-iCre infected neurons (Fig. 3.21 D left panel). Therefore, Cre-mediated deletion of Munc13-1 results in non-detectable Munc13-1 protein levels by Western blot analysis in vitro.

In order to assess physiological consequences of Cre-mediated Munc13-1 deletion in neurons, autaptic hippocampal neuron cultures from Munc13-1 Flp mice were infected with pFUGW-GFP or pFUGW-iCre lentivirus on DIV 2 and tested for their basic electrophysiological properties at different time points (Fig. 3.22 A-E). Electrophysiological experiments and analysis was performed by Dr. Riikka Liisa Uronen, Department of Molecular Neurobiology, Max-Planck Institute of Experimental Medicine, Göttingen, Germany. Already on DIV 10, a strong reduction in the measured excitatory postsynaptic current amplitude (EPSC, nA) and in the size of the readily-releasable pool (RRP, nC) were measured for cells infected with pFUGW-iCre (data not shown). Therefore data from recordings conducted on following days (DIV 12-16) from one culture were pooled for the analysis. The EPSC amplitude was dramatically reduced in cells infected with pFUGWiCre in comparison to pFUGW-GFP infected neurons (pFUGW-GFP: $3.141 \pm 0.5676$, $n=32$; pFUGW-iCre: $0.7037 \pm 0.1136, n=41 / P<0.0001,{ }^{* * *}$ ) (Fig. $\left.3.22 \mathrm{~A}, \mathrm{D}\right)$. Moreover the measured RRP size $(\mathrm{nC})$ was greatly diminished in pFUGW-iCre infected cells (pFUGWGFP: $376.0 \pm 75.91, n=17$; pFUGW-iCre: 75.41 \pm 11.31, $n=18 / \mathrm{P}=0.0003$, ***; Fig. $3.22 \mathrm{~B}$, E), with no significant differences in the calculated vesicular release probability (Pvr, \%) (pFUGW-GFP: $7.361 \pm 0.9006, n=17$; pFUGW-iCre: $8.672 \pm 1.387, n=18$ / $P=0.7791$, n.s.; Fig. $3.22 \mathrm{C})$.

Immuncocytochemical experiments of cultured neurons confirmed the biochemical and electrophysiological findings mentioned above. Immunofluorescent labelings of autaptic cells with primary antibodies directed against Munc13-1 protein infected with pFUGWGFP or pFUGW-iCre lentivirus and fixed on DIV 21 were performed. Neurons infected with pFUGW-GFP revealed close association of immunoreactivity for the active zone protein Munc13-1 (red) with immunoreactivity for the vesicular glutamate transporter VGLUT1 (green) along MAP-2 positive neuronal processes (grey) (Fig.3.22 F). Autaptic neurons infected with pFUGW-iCre were still able to form synapses, represented by the strong synaptic vesicle immunolabeling of VGLUT1. However Munc13-1 immunoreactivity was almost completely absent (Fig.3.22 G). Some background labeling was observed using the Munc13-1 primary antibody in both experimental conditions, most likely originating from the surrounding wild-type astrocyte cell layer since astrocyte cultures have been proposed to express Munc13-1 (Mungenast, 2011). 
A

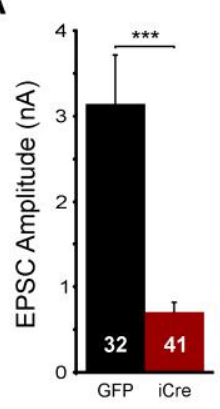

B

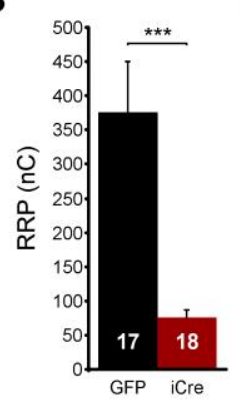

C

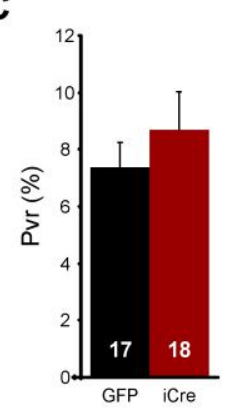

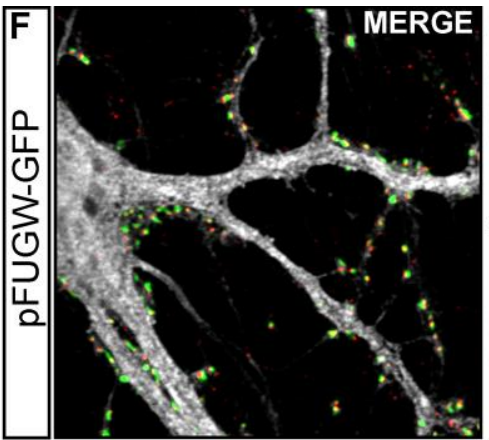

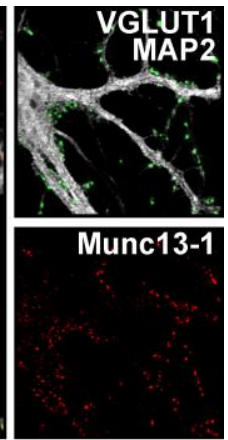

D

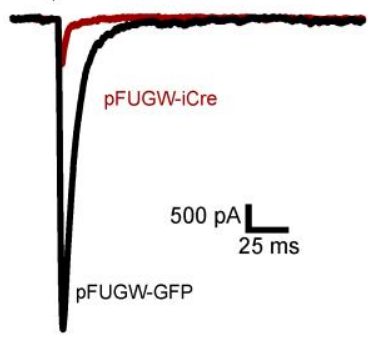

E

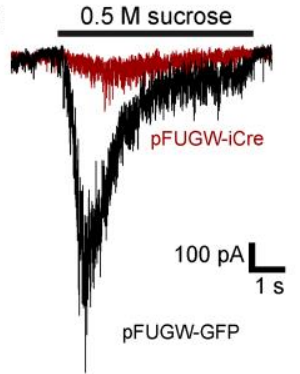

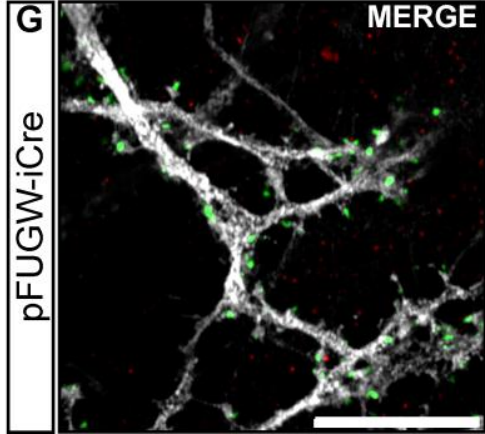

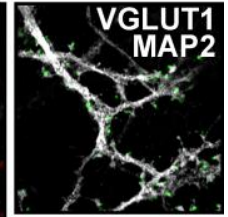

Munc13-1

Figure 3.22. Electrophysiological and morphological characterization of pFUGW-iCre infected Munc13-1 Flp hippocampal autaptic neurons

Physiological characteristics of autaptic excitatory hippocampal neurons infected at DIV 2 with either pFUGW-GFP or pFUGW-iCre lentivirus and whole-cell voltage clamped at DIV 12-16. A The Mean amplitude of the excitatory postsynaptic current (EPSC) resulting from an evoked action potential. B Mean size of the readily-releasable pool (RRP) after application of $0.5 \mathrm{M}$ hypertonic sucrose solution for 5 seconds. C Mean vesicular release probability (Pvr), as calculated by the ratio of charge evoked during a single action potential and that evoked during the sucrose application. D Example traces of evoked EPSC responses for neurons infected with pFUGW-GFP (black) or pFUGW-iCre (red). E Example traces for RRP release after stimulation with $0.5 \mathrm{M}$ sucrose in neurons infected with pFUGW-GFP (black) or pFUGW-iCre (red). F,G Immunolablling of Munc13-1 (red), MAP-2 (grey) and VGLUT1 (green) in pFUGW-GFP infected autaptic neurons (F, left panel) and in pFUGW-iCre infected autaptic neurons (G, left panel). Upper right panel: MAP-2 and VGLUT1 overlay, lower right panel: Munc13-1 immunolabelling. A-C: $n=$ number of cells from one culture (Mean + SEM), $\mathrm{P}<0.001$ : ${ }^{* *}$. Scale bar: G, $10 \mu \mathrm{m}$. Electrophysiological recordings were performed by Dr. R. L. Uronen. 


\section{Discussion}

4.1.

Methodological aspects in studying synaptic vesicle docking

4.1.1.

The organotypic slice culture system for studying lethal mouse mutants

All mouse mutants that were investigated in the course of this study die either pre- or perinatally. Despite this, multiple options were available to prepare brain samples for ultrastructural analysis by high-pressure freezing fixation and freeze substitution. The advantages gained by high-pressure freezing and freeze substitution, in terms of superior sample preservation compared to chemical fixation, are however offset by the need to work with small sample volumes, since freezing quality deteriorates with increasing sample size, introducing artifacts and perturbing cellular ultrastructure. The first option was to prepare acute slices of brain tissue as used for electrophysiological experiments, however this method is experimentally challenging for E18 - P0 mouse brains and the mechanical disruption of the slice surface introduced during sectioning bears the risk of inducing local excitotoxicity and stimulation of neuronal release in an unphysiological manner. However, surface areas of high-pressure frozen tissue are usually preferentially selected for ultrastructural studies, since they undergo extremely rapid cooling in proximity to external cryo-protectant during the freezing process and as a consequence, they typically exhibit improved ultrastructual preservation compared to the slice center. The second reason arguing against the acute slice preparation method was the fact that synaptic density in cortical regions, including the hippocampus, is very low at birth, which would have restricted the analysis to synapses in immature developmental stages and made a large-scale ultrastructural analysis of synaptic morphology more challenging.

I therefore decided to employ the hippocampal organotypic slice culture system for my purpose. Organotypic slice cultures from brain tissues, cultivated on small membrane inserts with the so-called interface method, provide established culture settings with a comparable rate of neuronal development and synaptogenesis to that of the intact tissue (reviewed in Gähwiler, 1997; De Simoni et al., 2003; Stoppini et al., 1991). Analysis of presynaptic protein $\mathrm{KO}$ mouse mutants has been successfully conducted by combining hippocampal organotypic slice culture preparations with electrophysiological experiments as well as ultrastructural analyses after cryo-fixation methods for electron microscopy (Kerr et al., 2008; Siksou et al., 2009; Zhao et al., 2012a). The main advantages of this culture system for our purpose were the fact that slice cultures could recover from cutting 
damage, permitting neurons and synapses to grow and develop in an in-situ-like environment. Moreover, slices thinned-out during four weeks in culture and became a perfect size to fit in a sample carrier for high-pressure freezing fixation. In comparison to dissociated neuron cultures, tissue slices are kept in a more physiological environment, which has been shown to extend the survival period of cultured neurons from mice carrying neurodegenerative mutations (Broeke et al., 2010; Heeroma et al., 2004). Hippocampal organotypic slices from mouse control and presynaptic protein mutants were therefore cultured for 4-5 weeks to guarantee a high synaptic density and to enable synapse maturation, also for mutant tissues that might suffer potential delays in neuronal development caused by the reduction or blockage of synaptic transmission, as has been reported for Munc13-1/2 DKO neurons (Broeke et al., 2010).

Using the combination of hippocampal organotypic slice cultures, high pressure freezing and freeze substitution, it became possible to obtain well-frozen samples with minimally perturbed ultrastructure. In control synapses from all genotypes, the vast majority of the vesicles within $40 \mathrm{~nm}$ of the active zone were indeed membrane-attached, indicating physiological, unstimulated conditions during the freezing process. Potential fusion intermediates, such as synaptic vesicles in hemifused or fully-collapsed states, were however only occasionally observed. Initially, freezing the samples in the presence of tetrodotoxin was considered to obtain an even more accurate approximation of the number of docked vesicles in non-stimulated neurons. Tetrodotoxin prevents action potential propagation along the neurons by blocking voltage-gated sodium channels. However, the homeostatic effects on neurons during and after pharmacological blockage of presynaptic transmission are not yet fully understood, and since our aim was to approach near physiological conditions during sample preparation, we decided against this option.

In summary, I showed that the hippocampal organotypic slice culture technique applied to lethal mouse mutants is a suitable experimental approach for cryo-fixation and ultrastructural EM analysis of synaptic parameters in a dense neuronal network.

\subsubsection{Studying synaptic vesicle docking using cryo-fixation methods for EM}

In recent years it has become evident that conventional chemical fixation methods for EM introduce serious problems that confound the study of synaptic vesicle docking at the active zone membrane, largely due to artifactual changes induced in cytoskeletal 
arrangements by the cross-linking of proteins and the dehydration of the sample (reviewed in Verhage \& Sørensen 2008) (See 1.4 Ultrastructural analysis of synaptic vesicle docking). Since then, many studies have aimed to prove that cryo-fixation methods offer a more realistic view of presynaptic ultrastructure and enable a more reliable analysis of synaptic vesicle membrane interactions at active zone release sites. The application of high-pressure freezing fixation and freeze substitution prior to plastic embedding and three-dimensional electron tomography revealed that synaptic vesicle membranes of docked synaptic vesicles are in direct contact with the active zone plasma membrane (Siksou et al., 2009).

Despite the improvements in synaptic ultrastructural preservation resulting from highpressure freezing, the imaging of samples at room temperature requires a cryosubstitution step in which sample water is slowly exchanged for an organic solvent at low temperatures and the sample is preserved by fixatives. In the present study, a combination of tannic acid and $\mathrm{OsO}_{4}$ was used, which fixes samples during the freeze substitution process and deposits a heavy metal precipitate on phospholipid bilayers, thereby enhancing membrane contrast for imaging (Giddings, 2003; Jiménez et al., 2009). The disadvantages of this dehydration and staining procedures include the potential destruction of cytoskeletal elements and the fact that imaging relies on the presence of the heavy metal membrane stain that might obscure small structures and thus limits the resolution during imaging.

Alternatively, cryo-preserved samples can be imaged at low temperatures by cryoelectron tomography, avoiding sample dehydration and staining. One disadvantage of this method is, however, that only plunge-frozen samples thinner than $500 \mathrm{~nm}$ can be imaged directly (e.g. synaptosomes), whereas thicker, high-pressure frozen specimens (e.g. organotypic slice cultures) require cryo-sectioning, which introduces severe compression artifacts. Moreover, unfixed samples are very beam-sensitive and imaging therefore requires a very low electron dose and a high defocus to obtain phase contrast of proteins and membranes. However, studies employing cryo-electron tomography after freezing of purified synaptosome preparations and organotypic slice cultures indicated that the vast majority of synaptic vesicles might not be in direct membrane to membrane contact with the active zone, but that the closest vesicles are rather linked to the plasma membrane by multiple short filaments, or tethers (Fernández-Busnadiego et al., 2010). These tethered vesicles are connected by an average of two or more short filaments to the membrane, a number that correlates nicely with the calculated number of SNARE complexes needed to overcome the energy barrier for fusion of synaptic vesicles in vivo (FernándezBusnadiego et al., 2010; Mohrmann and Sørensen, 2012). Moreover, these short tethers 
disappeared after the treatment of synaptotosomes with tetanus toxin, a molecule that cleaves free Synaptobrevin-2, supporting the idea that multiple partially, or fully assembled, SNARE complexes link synaptic vesicles to the membrane (FernándezBusnadiego et al., 2010). According to this criterion, vesicles in direct membrane contact would be classified as fusion intermediates. In the present study, I occasionally observed short filamentous structures between vesicles and active zone membranes, but the majority of the vesicles appeared to be in direct membrane contact. It is very likely that this discrepancy between our electron tomography approach on plastic sections and the cryo-electron tomography data is caused by the layer of precipitated stain on the phospholipid bilayers in my method. I was unable to quantify the number of short tethers in this study. Moreover, I termed vesicles that were in physical contact with the plasma membrane "membrane-attached" or "docked" synaptic vesicles, although in reality these vesicles might indeed be "tethered" by short tethers to the membrane.

Another possibility that may account for the lack of membrane-attached synaptic vesicles in the cryo-electron tomography study in comparison to my findings might be the differences in sample preparation prior to and during the cryo-fixation process. For cryo$E M$, rat synaptosomes were purified in a series of centrifugation steps prior to plungefreezing and results were compared with tomograms obtained from high-pressure frozen and cryo-sectioned organotypic slice cultures (Fernández-Busnadiego et al., 2010). In my preparations, I used $20 \%$ BSA dissolved into the culture medium for freezing. I found that the addition of BSA enabled excellent ultrastructural preservation over large areas without exposing the sample to a dramatic increase in osmolarity. In the study by FernandezBusnadiego and colleagues (2010), organotypic slices were frozen in the presence of $20 \%$ dextran and $5 \%$ sucrose after five minutes incubation at room temperature. This composition of cryoprotectant for freezing causes an increase of osmolarity to $\sim 600$ mOsm, which could trigger the fusion of primed vesicles and thus could easily reduce the number of readily-releasable vesicles in synapses and account for the almost complete lack of membrane-attached vesicles. Moreover, the physiological consequences of centrifugation during synaptosome preparation, and any potential impact this may have on the size of the RRP, is difficult to assess.

A recent study presented a fusion assay in which large liposomes containing stabilized Syntaxin-1/SNAP25 (1:1) complexes and liposomes containing full-length Synaptobrevin2 , or a deletion mutant $(\Delta 84)$ that prevents interactions at the last hydrophobic layer $(+8)$ of the four helical SNARE bundle preceding the C-terminal linker, enabled the capturing of different SNARE-dependent docking and fusion intermediates by cryo-electron microscopy (Hernandez et al., 2012). In this study Hernandez and colleagues showed that 
in the presence of $\Delta 84$ Synaptobrevin-2, liposomes can dock to each other. In this conformation the bilayers were described to be tightly held together 'without a resolvable space between the membranes' but fusion of liposomes was prohibited. This docked state was further described to require partial SNARE complex assembly. These findings indicate that in vitro, cryo-electron microscopy techniques can capture physical membrane-attachment at a stage in which SNARE complexes are partially engaged. However, in vivo partial SNARE complex zippering might be arrested further towards the $\mathrm{N}$-terminus by regulatory proteins, potentially explaining the observed gap and the short filamentous connections between synaptic vesicle and plasma membranes observed by Fernandez-Busnadiego and colleagues (Fernández-Busnadiego et al., 2010).

In summary, the possibility that electron tomography on plastic sections as employed in the present study approaches the limits of resolution when studying synaptic vesicle membrane-attachment cannot be excluded. Vesicles distanced less than $5 \mathrm{~nm}$ from the active zone membrane were therefore separated into two groups: (1) 0-2 nm and (2) 0-4 $\mathrm{nm}$. The 0-2 $\mathrm{nm}$ group contained all vesicles that appeared to be in direct membrane contact ("docked"). The 0-4 nm group included vesicles that were close enough for transSNARE interactions to occur (van den Bogaart et al., 2011; Li et al., 2007). The term "tethering" is used here primarily to describe membrane-proximal vesicles within $40 \mathrm{~nm}$ of the active zone that can interact with the membrane by long filaments. This synaptic vesicle tethering process has been shown to remain unaffected in Munc13-1/2 DKO synapses despite the loss of docked synaptic vesicles, a finding that we were able to confirm in the present study (Siksou et al., 2009).

\subsection{A common pool of docked and primed synaptic vesicles?}

Early EM studies that investigated the relationship between synaptic vesicle docking and priming were performed on chemically fixed wild-type neuron samples and compared the number of docked synaptic vesicles in electron micrographs with the number of released synaptic vesicles calculated from the measured postsynaptic response after hypertonic sucrose application (Murthy and Stevens, 1999; Rosenmund and Stevens, 1996; Schikorski and Stevens, 1997; Stevens and Tsujimoto, 1995). In these early studies, the number of membrane-attached synaptic vesicles correlated well with the number of fusion-competent synaptic vesicles in the sucrose pool. An alternative method to measure the RRP size of synaptic terminals is to apply a $20 \mathrm{~Hz}$ action potential stimulation protocol to successively release fusion-competent vesicles (Rosenmund and Stevens, 1996). 
Combining this protocol with subsequent FM1-43 membrane dye uptake, chemical fixation and photoconversion of the dye for electron microscopy, has again revealed a tight correlation between the numbers of retrieved and stained synaptic vesicles after pool depletion and the number of active zone membrane-attached synaptic vesicles. These findings support the hypothesis that the pool of physiological primed or fusion competent synaptic vesicles is comprised of morphological docked synaptic vesicles (Murthy and Stevens, 1999; Murthy et al., 1997, 2001; Schikorski and Stevens, 2001). Moreover, by coupling optical stimulation of neurotransmitter release in $C$. elegans neuromuscular junction synapses with rapid high-pressure freezing and electron microscopic imaging of plastic sections, it has been shown that the number of membrane-attached synaptic vesicles decreases after a stimulus, leading to the conclusion that morphologically docked vesicles are the first to fuse in response to an action potential (Watanabe et al., 2013).

In the present study, I demonstrated that in tomograms from all control groups analyzed, the vast majority of the vesicles within $40 \mathrm{~nm}$ of the active zone appeared to be in direct physical contact with the active zone membrane. This finding is very important, because it provides an explanation why in wild-type synapses the numbers of morphologically docked and functionally primed synaptic vesicles correlate well, irrespective of which fixation method or docking definition (direct attachment versus $40 \mathrm{~nm}$ bin) is applied.

\subsection{The role of the priming proteins Munc13/CAPS in synaptic vesicle docking}

\subsubsection{Munc13s}

First, the role of Munc13s in synaptic vesicle docking was reinvestigated in my experimental system. Munc13-1/2 DKO neurons were shown to exhibit a complete loss of physiologically primed synaptic vesicles as probed by application of hypertonic sucrose solution (Varoqueaux et al., 2002). In the present study, I found that synapses lacking both Munc13-1 and -2 are almost completely devoid of membrane-attached synaptic vesicles. Only one out of 15 tomograms harbored a total of three docked synaptic vesicles. Moreover, twelve synaptic profiles (80\%) lacked both docked and closely tethered vesicles (0-4 nm). In comparison to control synaptic profiles, the numbers of membrane-attached $(0-2 \mathrm{~nm})$ and closely tethered $(0-4 \mathrm{~nm})$ synaptic vesicles were reduced to $4 \%$ and $11 \%$, respectively. These findings are in line with previously published 
analyses of synaptic vesicle docking in $C$. elegans and mouse neurons in the absence of UNC-13/Munc13s (Gracheva et al., 2006, 2010; Siksou et al., 2009; Weimer et al., 2006). The remaining membrane-attached vesicles seen in the single tomogram may have been primed by an alternative priming mechanism. Munc13-independent priming has been reported in the mouse neuromuscular junction and in retina photoreceptor ribbon synapses, where synaptic vesicle priming and neurotransmitter release occurs even in the absence of Munc13 proteins (Cooper et al., 2012; Schmitz et al., 2001; Varoqueaux et al., 2005). However, no obvious morphological differences, indicative of a non-glutamatergic synapse, were observed in comparison to the remaining tomograms. Another explanation for the remaining docking could be the presence of an alternative Munc13 isoform in the imaged synapse. Munc13-3 mRNA has been detected by in situ hybridization in the mouse hippocampus. However the presence of Munc13-3 proteins in the hippocampus could not be demonstrated by Western blot analysis (Augustin et al., 1999b).

Recently, a study employing cryo-electron tomography on purified synaptosomes of RIM1 $\alpha$ KO mice, a well-established component of the active zone cytoskeleton and an interaction partner of Munc13-1 and ubMunc13-2, reported that RIM1 $\alpha$ might have a critical role in synaptic vesicle docking (Fernández-Busnadiego et al., 2013). As mentioned above, docked synaptic vesicles appeared to be in close proximity $(<5 \mathrm{~nm})$ to the plasma membrane in cryo-electron tomograms and to exhibit multiple short tethers in this configuration (Fernández-Busnadiego et al., 2010). In RIM1 $\alpha$ KO mice, five out of nine tomograms exhibited a vesicle number comparable to wild-type levels within $45 \mathrm{~nm}$ of the active zone membrane (KO-aligned) whereas the remaining synapses showed a decrease in the number of these membrane-proximal vesicles (KO-altered). Moreover, the number of synaptic vesicles within $5 \mathrm{~nm}$ of the plasma membrane was dramatically reduced, and KO-altered synaptic vesicles completely lacked multiple short tethers in the docked state. In addition to its roles in localizing $\mathrm{Ca}^{2+}$-channels to the active zone and in docking and priming synaptic vesicles in the vicinity of $\mathrm{Ca}^{2+}$-channels, $\operatorname{Rim} 1 \alpha$ has been implicated in the trafficking of Munc13s to synaptic targets (Andrews-Zwilling et al., 2006; Betz et al., 2001; Han et al., 2011; Kaeser et al., 2011, 2012). Of note, RIM1a KO mice exhibit a $60 \%$ reduction of combined Munc13 protein levels, whereas the genetic ablation of multiple RIM isoforms (Rim1 $\alpha, \operatorname{Rim} 1 \beta, \operatorname{RIM} 2 \alpha, \operatorname{RIM} 2 \beta$ and $\operatorname{RIM} 2 \gamma)$ causes a reduction of Munc13 levels by 67\% (Andrews-Zwilling et al., 2006; Deng et al., 2011; Kaeser et al., 2011; Schoch et al., 2002).

Consistent with these findings, Munc13 levels are also reduced in synaptosomes purified from RIM1 $\alpha \mathrm{KO}$ mice and quantitative analysis of immunofluorescence revealed a significant decrease in the frequency of colocalization between Munc13 and the active 
zone protein Bassoon (Fernández-Busnadiego et al., 2013). The described defects in vesicle priming and docking observed in RIM KO animals are therefore most likely attributable to reduced levels of Munc13s at the active zone. However, as it has been published previously, and has been demonstrated in the present study, loss of UNC13/Munc13 proteins causes an accumulation of synaptic vesicles close (8-10 nm), but not docked, to the active zone, explaining why the number of vesicles within $40 \mathrm{~nm}$ of the active zone membrane remains unchanged in Munc13-1/2 DKO synapses and a docking deficit could not have been detected in electron microscopic analyses using conventional chemical fixation (Gracheva et al., 2010; Siksou et al., 2009). The absence of a prominent membrane-proximal vesicle accumulation in $44 \%$ of RIM1 $\alpha$ synaptosomes may indicate an additional, Munc13 independent role for RIM proteins in tethering vesicles close to release sites in a process upstream of docking/priming (Fernández-Busnadiego et al., 2013).

The molecular mechanism by which Munc13s dock/prime synaptic vesicles is not yet fully understood. The C-terminally located MUN domain present in all Munc13 isoforms includes two MHD domains and is considered essential for Munc13 priming activity (Basu et al., 2005; Stevens et al., 2005). The MUN domain mediates Munc13 binding to Syntaxin-1 and Munc13s could therefore induce a switch from the closed to an open Syntaxin-1 conformation, which would enable and/or accelerate SNARE complex nucleation (Betz et al., 1997; Ma et al., 2011, 2013; Sassa et al., 1999). Although the interaction between Munc13 and Syntaxin-1 is weak in comparison to the tight binding between Munc18-1 and Syntaxin-1, it can accelerate the transformation from Syntaxin1/Munc18-1 complexes to fully assembled SNARE complexes in in-vitro assays (Ma et al., 2011, 2013; Sassa et al., 1999). The expression of a constitutively open form of UNC-64, the $C$. elegans homologue of Syntaxin-1, in the UNC-64/UNC-13 DKO background can rescue the massive deficits in neurotransmission and synaptic vesicle docking in cryopreserved samples observed in UNC-13 null mutants (Hammarlund et al., 2007; Richmond et al., 2001). Crossing the Munc13-1 KO mouse line with a knock-in mouse line that expresses the constitutively open form of Syntaxin-1, however, failed to rescue the perinatal lethality of Munc13-1 KO mice (Gerber et al., 2008). In C. elegans, loss of UNC18 or UNC-64 (Syntaxin-1) was described to result in a loss of docked and membraneproximal, or "tethered", synaptic vesicles in cryo-preserved samples, whereas UNC13/Munc13-deficient animals were still able to accumulate synaptic vesicles close to the release sites, but not to dock these at the membrane as confirmed in the present study (Gracheva et al., 2010). These findings indicate that Syntaxin-1/Munc18 interactions may tether vesicles close to the membrane, with Munc13s having a downstream role in 
synaptic vesicle docking, possibly by opening Syntaxin-1 for SNARE complex formation (Gracheva et al., 2010). However, due to the severe neurodegenerative phenotype of Munc18-1 KO mice, I could not perform a docking analysis of Munc18-1 KO synapses in my experimental setting.

\subsection{2. $\quad$ CAPS}

Next, the role of CAPS proteins in synaptic vesicle docking was investigated by our high resolution three-dimensional EM approach. The ultrastructural analysis of synaptic vesicle docking revealed that $62 \%$ of the sampled synaptic profiles (12 out of 19 tomograms) were completely devoid of membrane-attached synaptic vesicles (0-2 nm). Moreover, $58 \%$ of all CAPS-1/2 DKO synaptic profiles (11 out of 19 tomograms) also lacked vesicles closely tethered to the active zone membrane (0-4 nm). Electrophysiological analyses of autaptic neurons lacking both mammalian CAPS isoforms revealed that $38 \%$ of neurons measured were completely devoid of primed synaptic vesicles as determined by hypertonic sucrose application, whereas in the remaining $64 \%$ of the cells, the size of the RRP was decreased by $85 \%$ in comparison to controls (Jockusch et al., 2007). Due to the fact that only subvolumes of presynaptic terminals were tomographically reconstructed, it is likely that the number of neurons/synapses capable of priming synaptic vesicles is underestimated in the present study. In comparison to control synapses, in tomograms from CAPS-1/2 DKO synapses that still harbored membrane-attached synaptic vesicles, the number of docked (0-2 nm) and closely tethered (0-4 nm) vesicles was reduced by $81 \%$ and $72 \%$, respectively. The $81 \%$ reduction in morphological synaptic vesicle docking for CAPS DKO synaptic profiles correlates closely with the observed $85 \%$ reduction in the RRP size measured by hypertonic sucrose application in release-active autaptic neurons (Jockusch et al., 2007). These findings support the hypothesis that functional priming and morphological docking are correlates of the same molecular process. No significant changes in the basic synaptic morphology of CAPS-deficient neurons were observed, therefore an indirect effect of impaired LDCV release, is unlikely to account for the defect in synaptic vesicle release. In this context, my data also failed to reveal an accumulation of LDCVs in the presynaptic terminals of neurons lacking CAPS proteins, in contrast to what has been described for Drosophila neuromuscular junction synapses lacking CAPS (Renden et al., 2001). Renden et al., observed that, in addition to the defects observed in LDCV secretion, the release of glutamatergic synaptic vesicles at the neuromuscular junction was greatly impaired. Since the observed effects on synaptic vesicle release in CAPS mutants could be rescued by transgenic expression of rat CAPS in all neurons, but 
not in single motoneurons, the authors speculated that the presynaptic transmission deficit was an indirect consequence of the altered release of LDCV cargo (i.e. neuromodulators). One possibility to assess whether the observed defects in synaptic vesicle docking/priming are indeed indirect would be to analyze CAPS-1/2 DKO neurons in a micro-network cell culture system, in which neurons are spread in a slightly higher density as compared to the classical autaptic neuron culture, thus allowing two isolated neurons to form a combination of autaptic and interneural synapses on an astrocyte island (Liu et al., 2013). Similar to the single motoneuron-rescue experiments in Drosophila larvae by Renden et al., co-culturing of CAPS-1/2 DKO and wild-type neurons followed by paired recordings of autaptic and heterosynaptic responses after stimulation of the CAPS DKO cell could give an insight about possible pre- and postsynaptic effects caused by, for example, altered LDCV release from neighboring cells.

Release from mammalian autaptic neurons with no, or dramatically reduced, RRP sizes was rescued by viral expression of CAPS-1 or high-frequency stimulation (Jockusch et al., 2007). Moreover, $\mathrm{Ca}^{2+}$-uncaging in silent CAPS-1/2 DKO neurons revealed that CAPS deficient cells were able to release, albeit with a significant delay in comparison to control cells (Burgalossi et al., 2010). The latter finding indicates that an increase in intracellular $\mathrm{Ca}^{2+}$ can rescue synaptic transmission in CAPS1-/2 DKO neurons, indicating that the basic release machinery for synaptic vesicle release is functionally intact. Therefore CAPS may have an important function in vesicle priming at low $\mathrm{Ca}^{2+}$-concentrations, and elevations in residual intracellular $\mathrm{Ca}^{2+}$ resulting from high-frequency stimulation may overcome the CAPS1-/2 DKO phenotype via activation of for example Munc13 proteins, which possess multiple $\mathrm{Ca}^{2+}$-sensitive domains $\left(\mathrm{C}_{2} \mathrm{~B}\right.$ domain, $\mathrm{Ca}^{2+}$-Calmodulin binding site) (Jockusch et al., 2007). In contrast to Munc13-1/2 DKO synapses, synaptic vesicles in CAPS-deficient terminals did not accumulate at a distance of $8-10 \mathrm{~nm}$ from the active zone membrane, but rather appeared to be randomly distributed within $40 \mathrm{~nm}$ from the active zone membrane. In chemically fixed CAPS-deficient cells, a slight, but not statistically significant, decrease in the number of docked vesicles was reported, a phenotype attributable to the absence of a membrane-proximal synaptic vesicle accumulation (Jockusch et al., 2007). The defect in synaptic vesicle docking in the absence of members of the CAPS family could indeed explain the delay in the response after $\mathrm{Ca}^{2+}$-uncaging, which likely reflects $\mathrm{Ca}^{2+}$-dependent priming and fusion of newly primed vesicles (Burgalossi et al., 2010).

In addition to the effects of high $\mathrm{Ca}^{2+}$-concentrations on release in CAPS-1/2 DKO cells, neurons that exhibited no or dramatically reduced evoked release were found to be rescued by treatment with $\mathrm{PDBu}$, a phorbol ester capable of activating Munc13 proteins 
and other proteins via their $\mathrm{C}_{1}$ domain. However, the exact nature of the relationship between Munc13 and CAPS proteins in synaptic vesicle priming has yet to be assessed experimentally as so far cross-rescue experiments overexpressing Munc13s in CAPSdeficient cells, and vice versa, failed to rectify the deficit in synaptic vesicle release (Jockusch et al., 2007).

The molecular mechanism underlying CAPS-dependent synaptic vesicle priming is not fully understood. CAPS stimulates formation of trans-SNARE complexes from Syntaxin1/SNAP25 acceptor and Synaptobrevin-2 donor liposomes in in vitro fusion assays (James et al., 2008, 2009, 2010). In these studies, acceleration of trans-SNARE complex formation was highly $\mathrm{PIP}_{2}$-dependent and required binding to Syntaxin-1. Recently it was shown that CAPS can bind the t-SNAREs SNAP25 and Syntaxin-1 with high affinity in the SNARE motif, but that it can interact with Synaptobrevin-2 only weakly at the N-terminus, which has been interpreted as a potential role of CAPS to promote $\mathrm{N}$ - to C-terminal SNARE complex zippering (Daily et al., 2010). The region that promotes SNARE-complex assembly corresponds to the MHD domain, which has been proposed to mediate the conformational switch from closed to open Syntaxin-1 (Khodthong et al., 2011). Recent evidence indicates that CAPS-1 preferentially binds the open form of Syntaxin-1 by targeting the full length of the cytoplasmic domain of Syntaxin-1, whereas Munc13-1 interacts with the first 80 amino acid residues of Syntaxin-1, therefore binding the closed conformation of Syntaxin-1 (Parsaud et al., 2013). The authors argue that Munc13-1 possibly opens Syntaxin-1, which becomes then stabilized by CAPS prior to SNARE nucleation, which is in line with previous publications proposing a sequential mode of action for Munc13s and CAPS proteins in secretory vesicle priming (Jockusch et al., 2007; Liu et al., 2010; Parsaud et al., 2013). LDCV release from mouse chromaffin cells lacking CAPS proteins is heavily impaired, however only minor changes in the number of docked vesicles had been observed for CAPS-1 KO cells and no differences to control cells for CAPS-1/2 DKO cells (Liu et al., 2010a; Speidel et al., 2005). Also overexpression of Munc13-1 in chromaffin cells did not change the number of morphologically docked LDCVs, but led to an increase in the measured RRP size (Ashery et al., 2000). Overexpression of a constitutive open form of Syntaxin-1 in CAPS-1/2 DKO chromaffin cells can rescue the reduced electrophysiologically assessed readily-releasable LDCV pool size (Liu et al., 2010). In C. elegans, open Syntaxin can rescue the LDCV docking deficits observed in UNC-31 (CAPS) and the synaptic vesicle docking and priming deficit described for UNC-13 KO worms (Hammarlund et al., 2007, 2008; Richmond et al., 2001). All these findings indicate that CAPS proteins have a role in secretory vesicle priming and that they are very likely to act in the same molecular pathway as Munc13s. 
In summary, the findings of the present study demonstrate that the observed defects in synaptic vesicle priming upon loss of the Munc13 and CAPS proteins correlate nicely with an inability to dock synaptic vesicles to the active zone membrane. Therefore functional synaptic vesicle priming and morphological synaptic vesicle membrane-attachment may indeed describe the same mechanism rendering synaptic vesicles fusion competent. In the following, I will therefore use the terms docking and priming interchangeably for describing the final membrane-attachment process that renders synaptic vesicles fusioncompetent (docking/priming).

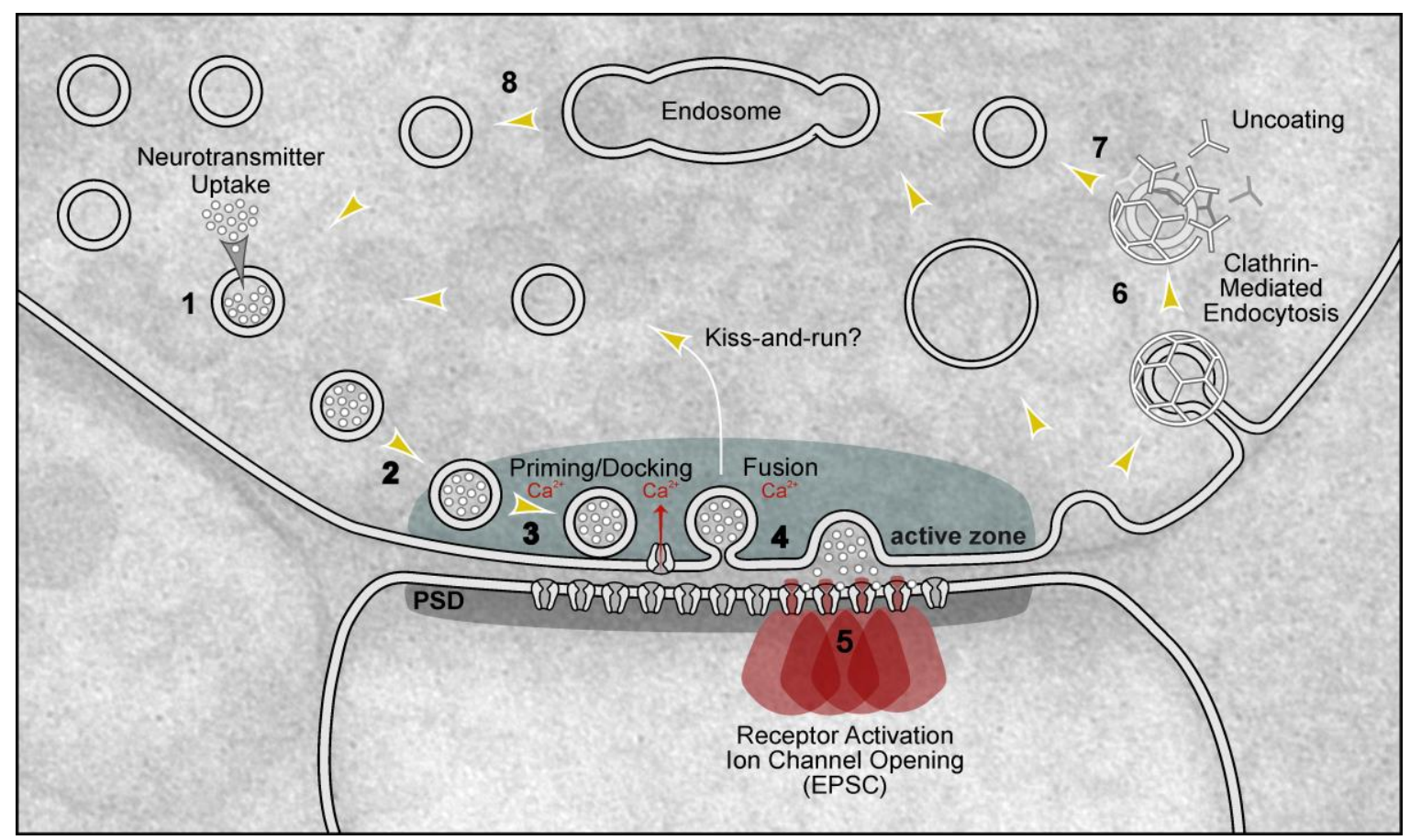

Figure 4.1. The synaptic vesicle cycle at excitatory synapses

Synaptic vesicles are locally recycled in presynaptic terminals. Synaptic vesicles are filled with the neurotransmitter (here e.g. glutamate) (1) and translocated to the active zone. There, synaptic vesicles become potentially tethered to the plasma membrane (2) and undergo a $\mathrm{Ca}^{2+}$-regulated docking/priming process to acquire fusion-competence (3). In response to the arrival of an action potential and an increase in $\mathrm{Ca}^{2+}$-concentration, docked/primed synaptic vesicles can fuse with the plasma membrane (4) and release their neurotransmitter content into the synaptic cleft. Neurotransmitter molecules diffuse through the synaptic cleft to mediate receptor ion channel opening and allow ion influx generating an excitatory postsynaptic current (EPSC) (5). Synaptic vesicle membranes are recycled in a process called endocytosis (6). The best-described pathway is clathrin-mediated recycling, in which clathrin-coated vesicles bud off from the membrane, become uncoated and fuse with early endosomes (7). Kiss-and-run exocytosis and bulk membrane retrieval are possible alternative, clathrin-independent recycling routes. Schematic provided by Dr. B. Cooper; adapted from (Jahn and Fasshauer, 2012). 
4.4 .

A role for all three neuronal SNAREs in synaptic vesicle docking and priming

As discussed above, priming by Munc13s and CAPS proteins most likely involves interactions with Syntaxin-1 and/or the SNARE complex. Therefore, the individual SNAREs and/or trans-interactions between the v-SNARE Synaptobrevin-2 and the tSNAREs Syntaxin-1 and SNAP25 have long been predicted to function in synaptic vesicle priming or docking (Pevsner et al., 1994).

Early studies using genetic knock-out strategies or proteolytic cleavage of SNARE proteins in neurons from different species including C. elegans, Drosophila, and mice revealed severe reductions in measured RRP sizes, but the number of membrane-docked synaptic vesicles was unchanged (Broadie et al., 1995; Deák et al., 2004; Gerber et al., 2008; Hunt et al., 1994; de Wit et al., 2006). Since early studies on KO animals for UNC$13 /$ Munc13 priming proteins failed to reveal changes in the number of membrane-attached synaptic vesicles in neurons in chemically fixed EM samples, it was assumed that synaptic vesicle membrane-attachment might be a prerequisite for vesicle priming and fusion, but that docking alone is most likely not sufficient to render a vesicle fusioncompetent (Richmond et al., 1999; Varoqueaux et al., 2002). Based on this model, priming and SNARE complex nucleation were believed to occur in a post-docking step and most likely required the opening of UNC-64/Syntaxin-1 by members of the UNC13/Munc13 family (Richmond et al., 2001). Whether partial or full SNARE complex formation could indeed be the molecular basis of synaptic vesicle priming and whether a semi-zippered SNARE complex could be stabilized sufficiently prior to fusion in such a scenario has been often controversially discussed (reviewed in Jahn \& Fasshauer 2012; Rizo \& Südhof 2012). In the present study, I aimed to analyze the effects of genetic deletion of the neuronal SNARE proteins Syntaxin-1, SNAP25 and Synaptobrevin-2 on synaptic vesicle docking for the first time in the same experimental setting. It has been shown that the individual t- and v-SNARE molecules on opposing membrane bilayers start interacting with their SNARE motifs at a distance of $8 \mathrm{~nm}$ and SNARE zippering arrests at a distance of around 2-4 $\mathrm{nm}$, with $2 \mathrm{~nm}$ being the thickness of the fully assembled SNARE bundle (Li et al., 2007; Poirier et al., 1998; Sutton et al., 1998). Förster resonance energy transfer (FRET) signal caused by SNARE complex assembly of $t$ - and v-SNAREs during liposome fusion has been calculated to start at a distance smaller than $5 \mathrm{~nm}$ between membranes (van den Bogaart et al., 2011). With the combination of cryo-fixation, freezesubstitution and electron tomography used in the present study, I was confident that it would be possible to resolve membrane distances smaller than $5 \mathrm{~nm}$ (See 4.1.2.). Indeed, 
in my high-resolution electron microscopy analysis of cryo-preserved samples, I found that all three neuronal SNARE proteins are involved in the synaptic vesicle docking process, since in the absence or upon reduced expression of the individual SNARE proteins, synaptic vesicles were greatly impaired in their ability to physically contact the active zone plasma membrane in excitatory synapses. Moreover, an accumulation of vesicles close to the plasma membrane, but not in direct contact with the active zone was detected in Synaptpbrevin-2 and SNAP25 KO neurons. These findings support the idea that synaptic vesicle docking/priming is mediated by Munc13 and CAPS proteins and involves (partial) SNARE complex assembly.

\subsection{Loss of individual SNARE proteins can be partially compensated}

In the present study, I observed docking deficits for neurons lacking the individual SNARE proteins. The greatest reduction in synaptic vesicle membrane-attachment was observed for SNAP25 KO neurons. 13 out of 25 tomograms from SNAP25 KO synapses (52\%) were completely devoid of membrane-attached vesicles $(0-2 \mathrm{~nm})$ with 7 out of 25 tomograms (28\%) lacking both, physically docked and closely tethered synaptic vesicles (0-4 nm). Synapses lacking SNAP25 showed a reduction in the numbers of membraneattached and closely tethered vesicles to $21 \%$ and $35 \%$ of controls, respectively. In low density cultures from SNAP25 KO neurons, cells degenerated during culturing, but survival was prolonged by plating cells at high densities. In such a continental culture setting, it has been described that $20-30 \%$ of SNAP25 KO neurons did not exhibit action potential- evoked release. In the remaining cells only very small EPSC amplitudes could be measured and spontaneous release and the RRP size probed by hypertonic sucrose solution was found to be reduced to $14 \%$ and $10 \%$ of controls, respectively (Bronk et al., 2007).

The fact that the pool of readily releasable fusion-competent and docked vesicles as described here are not completely absent, but rather reduced, raises the possibility that alternative SNARE proteins compensate for the loss of SNAP25 (Bronk et al., 2007). One such candidate SNARE molecule is SNAP23, a neuronally expressed SNAP25 homologue capable of rescuing sucrose-evoked, but not synchronous action potentialevoked, release in cultured hippocampal neurons (Delgado-Martínez et al., 2007). Recently, SNAP23 has been described to reside specifically in postsynaptic terminals, where it has a functional role in N-methyl-D-aspartate (NMDA) receptor exocytosis (Suh et al., 2010). Consistent with the findings of Suh et al., no specific co-localization was 
detected between the glutamatergic vesicle marker VGLUT1 and SNAP23, which appeared rather in apposition to excitatory terminals in organotypic hippocampal slices. Based on its localization, it is unlikely that SNAP23 can compensate for SNAP25 and account for the remaining docked vesicles observed in SNAP25 KO synapses. Other possible candidates and SNAP25 homologues that are expressed in the brain are SNAP29 and SNAP47 (Holt et al., 2006; Steegmaier, 1998). SNAP29 lacks the membrane anchor present in SNAP25 and SNAP23, but is present on multiple membranes and can bind to a broad range of Syntaxins, including Syntaxin- $1 \mathrm{~A}$ (Steegmaier, 1998). SNAP29 has, however, been proposed to function as an inhibitor of synaptic transmission (Pan et al., 2005). SNAP47 also lacks a membrane anchor, but is enriched on intracellular membranes and can form SNARE complexes with Synaptobrevin-2 and Syntaxin-1 to mediate proteoliposome fusion in vitro (Holt et al., 2006). More recently, SNAP-47 has been described to function in postsynaptic AMPA ( $\alpha-$ Amino-3-hydroxy-5-methyl-4-isoxazolepropionic acid) receptor exocytosis during longterm potentiation (Jurado et al., 2013). It would be interesting to investigate whether changes in the expression level or localization of these alternative SNAP25 homologues might explain the residual synaptic vesicle docking and neurotransmitter release in SNAP25 deficient cells.

In comparison to controls, Syntaxin-1A / Syntaxin-1B $\mathrm{B}^{\mathrm{YFP}}$ neurons exhibited a reduction in the numbers of membrane-attached $(0-2 \mathrm{~nm})$ and closely tethered $(0-4 \mathrm{~nm})$ vesicles by $38 \%$ and $35 \%$, respectively. Previous electrophysiological analyses in hippocampal autaptic neurons of these mutant had revealed a $65 \%$ reduction of the RRP size and an $80 \%$ reduction of EPSC size compared to controls (Arancillo et al., 2013). In comparison to the severe electrophysiological deficits in terms of the number of primed synaptic vesicles, I only detected milder effects on the number of membrane-attached synaptic vesicles. This discrepancy can most likely be attributed to the differences in the culture system, since organotypic slices were cultured for up to five weeks in a dense neuronal network, which is considerably longer than is possible with autaptic cell cultures. It is likely that during this extended culture period, compensatory, likely activity-dependent effects result in stabilization of the remaining Syntaxin-1B ${ }^{\text {YFP }}$ molecules, potentially via cell homeostatic mechanisms functioning to protect Syntaxin-1B ${ }^{\mathrm{YFP}}$ from degradation. A recent study suggested that expression of Syntaxin-2, -3 and -4 , which are BoNT/C resistant, can rescue neuronal cell death induced by proteolytic cleavage of Syntaxin-1 (Peng et al., 2013). However, attempts to culture Syntaxin-1A/B DKO neurons in an organotypic slice culture system failed in my hands and neurons from this genotype died within the first 
couple of days, indicating that most likely no other Syntaxin isoform can compensate for the loss of Syntaxin-1 in neurons in culture.

The analysis of synaptic vesicle docking in Synaptobrevin-2 KO neurons revealed reductions in the numbers of membrane-attached (0-2) and closely tethered (0-4 nm) vesicles by $33 \%$ and $43 \%$, respectively, compared to control samples. In a mass culture system, all Synaptobrevin-2 KO neurons were described to be able to release. However, evoked and spontaneous release were strongly impaired and the RRP size measured after hypertonic sucrose application was reduced to $10 \%$ of control levels (Schoch et al., 2001). Here, it was noticed that $33 \%$ of Synaptobrevin-2 KO synapses seemed to be less affected by the loss of the v-SNARE, since they exhibited comparable numbers of membrane-attached synaptic vesicles as control synapses, whereas the numbers of membrane-attached and closely-tethered vesicles in the remaining $\mathrm{KO}$ synapses were reduced by $95 \%$. By immunohistochemical stainings, I demonstrated that Synaptobrevin1, another Synaptobrevin isoform expressed in the brain (Takamori et al., 2006), has been upregulated in glutamatergic synapses in comparison to control slices. In a previous study, Synaptobrevin-1 was not detected by Western blot analysis in P0 Synaptobrevin-2 KO mouse brain homogenates (Schoch et al., 2001). However, in situ hybridization experiments mapping the developmental pattern of Synaptobrevin-1 expression in the hippocampus have demonstrated that this Synaptobrevin isoform is first expressed at around P14 (Allen Developing Mouse Brain Atlas). In the present study, a striking increase in the number of glutamatergic synapses that exhibited Synaptobrevin-1 immunoreactivity from $8 \%$ in control to $36 \%$ in Synaptobrevin-2 KO organotypicic slices was demonstrated. This finding was compelling, since control samples expressed Synaptobrevin-1 preferentially at non-glutamatergic synapses, with the majority of Synaptobrevin-1 puncta not colocalizing with VGLUT1. The presence of Synaptobrevin-1 in $36 \%$ of all glutamatergic synapses correlated well with the observation that $33 \%$ of the analyzed tomograms exhibited normal synaptic vesicle docking. It can therefore be postulated that loss of Synaptobrevin-2 is at least partially compensated for by an upregulation of Synaptobrevin-1. The question as to whether Synaptobrevin-1 can rescue the physiological deficits observed in Synaptobrevin-2 KO neurons has not previously been addressed to my knowledge. However, it was not possible to detect Synaptobrevin-1 in all glutamatergic terminals, explaining the presence of Synaptobrevin-2 KO synapses that harbored only very low numbers of docked synaptic vesicles. It cannot be excluded that additional Synaptobrevin homologues may also compensate for the loss of Synaptobrevin-2. A promising candidate is VAMP7, a tetanus toxin-insensitive Synaptobrevin-2 homologue that has been shown to reside in presynaptic terminals in the 
brain, e.g. in the hippocampal mossy fiber synapses, and has been implicated in asynchronous synaptic vesicle release (Hua et al., 2011b; Muzerelle et al., 2003; Scheuber et al., 2006).

In conclusion, the presence of a small pool of docked synaptic vesicles in the absence of the major synaptic SNARE molecules could be attributed to a compensatory effect by other SNARE isoforms or homologues. Above all, the majority of SNAP25 KO neurons have been shown to degenerate in culture, indicating that survival of the remaining neurons depends on an alternative SNARE isoform. Indeed, such a compensatory effect appears to operate in Synaptobrevin-2 deficient samples, in which an upregulation of Synaptobrevin-1 can rescue normal vesicle docking in a subset of glutamatergic synapses.

\subsection{Increased vesicle sizes do not cause the deficiency in synaptic vesicle membrane-attachment}

In the present study, a significant increase in synaptic vesicle size was detected in Munc13-1/2, SNAP25 and Synaptobrevin-2 KO synapses, genotypes that also exhibited severe deficits in synaptic vesicle membrane-attachment. To address the possibility that synaptic vesicle docking at the active zone is inhibited as a consequence of increased vesicle size, the vesicle diameters of membrane-attached synaptic vesicles in $\mathrm{KO}$ genotypes were measured and compared to those in respective controls. Thereby it could be confirmed that synaptic vesicles of a larger diameter were still able to reach the active zone membrane in all three mentioned genotypes. Moreover, analysis of synaptic vesicle

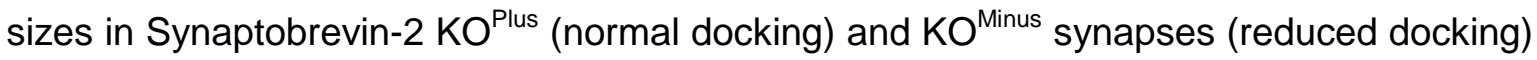
revealed that both groups exhibited significant increases in synaptic vesicle volumes. Since previous work has shown that short-term treatment of rat synaptosomes with tetanus toxin results in smaller synaptic vesicle sizes, the observed increases in vesicle size observed in the present study are likely a consequence of altered membranetrafficking caused by the permanent block of release (Fernández-Busnadiego et al., 2010). Docking intermediates formed by large liposomes in in vitro assays have been found to be more stable than those of smaller liposomes (Hernandez et al., 2012). If the increased synaptic vesicle size displayed by Munc13-1/2 DKO, SNAP25 KO and Synaptobrevin-2 KO mutants had a negative impact on the fusogenicity of synaptic vesicles, an accumulation, rather than depletion, of docked/primed vesicles at the active zone membrane would be expected, in direct contrast to the results of the present study. 


\subsection{Can trans-SNARE complexes be stabilized in vivo?}

Based on my data, it can be concluded that the neuronal SNARE proteins play an integral role in synaptic vesicle docking/priming in neurons. The findings presented in this study are important in the context of the ongoing discussion whether stable completely or partially assembled trans-SNARE complexes exist prior to vesicle fusion (reviewed in Jahn and Fasshauer 2012; Rizo and Südhof 2012).

Central to this discussion is the problem that trans-SNARE complexes seem to be difficult to capture and to stabilize in vitro. Studies that used reconstituted SNARE complex formation assays led to a model according to which the two t-SNAREs Syntaxin-1 and SNAP25 form a highly reactive intermediate acceptor complex on the membrane prior to Synaptobrevin-2 engagement and full SNARE complex assembly (Fasshauer and Margittai, 2004; Pobbati et al., 2006). This t-SNARE acceptor complex has to be stabilized in vitro to prevent it from forming a dead-end complex, which results from the interaction of two SNARE motifs from two Syntaxin-1 molecules and two SNARE motifs from one SNAP25 molecule. Rendering a stable Syntaxin-1/SNAP25 (1:1) complex in vitro can be achieved after the addition of a Synaptobrevin-2 peptide that is comprised of the Cterminal part of the SNARE motif, but lacks its N-terminus $(\Delta N)$ (Hernandez et al., 2012). There is, however, evidence from liposome and single vesicle fusion assays that indicates that different SNARE-mediated docking and fusion intermediates can be distinguished and that trans-SNARE complexes may be stabilized prior to membrane fusion (Bowen et al., 2004; Cypionka et al., 2009; Kyoung et al., 2011).

A more physiological approach to demonstrate experimentally the relationship between the physiological priming step and molecular SNARE interactions was undertaken by Sørensen and colleagues (Sørensen et al., 2006; Walter et al., 2010; Weber et al., 2010; Wiederhold et al., 2010). In these studies, mutations were introduced at different positions within the SNARE motifs of SNAP25 and Synaptobrevin-2 and the physiological effects were tested by overexpression of the respective constructs in chromaffin cells and/or neurons from respective null mutants. The authors consistently demonstrated that prevention of C-terminal SNARE zippering, by mutation of amino acid residues in the last hydrophobic C-terminal layers of the SNARE motif, decreases the ability of membranes to fuse both in vitro and in vivo. Abolishing N-terminal SNARE interactions, however, diminished the secretory vesicle priming efficiency in chromaffin cells with only minor effects in neurons. In this model, N-terminal interactions of the SNAREs define the primed synaptic vesicle state, whereas full C-terminal SNARE zippering is required for triggering 
membrane fusion. These findings imply that stable partially N-terminal assembled SNARE complexes might indeed underlie the primed synaptic vesicle state. A recent study using the Synaptobrevin-2 C-terminal residue deletion mutant $\Delta 84$ in a liposome fusion assay demonstrated that liposome docking, defined by direct bilayer-bilayer contact, was unaffected in cryo-electron micrographs, whereas fusion was greatly abolished in vitro (Hernandez et al., 2012). The fact that $\mathrm{N}$-terminal SNARE interactions allow vesicle priming (Sørensen et al., 2006; Walter et al., 2010; Weber et al., 2010; Wiederhold et al., 2010) and docking (Hernandez et al., 2012) supports the hypothesis that partial SNARE assembly underlies the docked vesicle state.

Taken together, there is evidence to support the hypothesis that stable neuronal transSNARE complexes exist at an intermediate step prior to vesicle fusion. Consistent with this view, my data demonstrate a loss of membrane-attached synaptic vesicles within 0-4 $\mathrm{nm}$ of the active zone membrane in SNARE-deficient neurons. The finding that interruption of SNARE zippering at the level of the last hydrophobic layers preceding the linker to the TMRs arrests vesicles in a primed/docked state, but prevents membrane fusion, appears to strengthen the validity of this model. It is therefore very likely that (partial) SNARE complex assembly is the molecular mechanism underlying synaptic vesicle docking and priming.

\subsection{Can partially assembled trans-SNARE complexes be stabilized by Synaptotagmin-1 or Complexins?}

In the present study, it was demonstrated that Munc13 and CAPS proteins prime synaptic vesicles by enabling them to dock to the plasma membrane in a SNARE-dependent manner. In this docked/primed and fusion-competent synaptic vesicle state, neuronal SNARE proteins possibly form (partially) assembled SNARE complexes in order to secure fast C-terminal SNARE motif zippering in the presence of $\mathrm{Ca}^{2+}$ to trigger membrane fusion. In vitro, stabilization of the Syntaxin-1/SNAP25 (1:1) acceptor complex can be achieved by the presence of the Synaptobrevin-2 $\Delta \mathrm{N}$, which will bind to the C-terminal SNARE motives of SNAP25 and Syntaxin-1, but leaves a free $\mathrm{N}$-terminal binding site for full length Synaptobrevin-2 (Hernandez et al., 2012). Upon full-length Synaptobrevin-2 engagement, zippering of the SNARE complex has been shown to slow down during the replacement of the first $\mathrm{N}$-terminal layers of Synaptobrevin-2 $\Delta \mathrm{N}$, but then rapidly resume towards the C-terminus (Hernandez et al., 2012). Whether, and if so, how partially assembled SNARE complexes are stabilized in vivo remains unclear. 
Prime candidates for performing such a role are Synaptotagmin-1 and Complexins, based on their ability to interact with the SNARE complex. Complexins can bind via a central $\alpha$ helix to the assembled SNARE complex in a groove formed by the SNARE motifs of Syntaxin-1 and Synaptobrevin-2 (Chen et al., 2002). Previous analyses from Complexin1/2 DKO and Complexin-1/2/3 TKO mice revealed a reduction in evoked release, but no corresponding changes in RRP size, thus resulting in a decreased vesicular release probability (Reim et al., 2001; Xue et al., 2007, 2008).

Moreover, genetic deletion of the major mouse Complexin isoforms had no impact on the mEPSC frequency in these studies. Based on these findings, it has been proposed that binding of Complexins to the SNARE complex might facilitate synaptic vesicle release (reviewed in Brose 2008). In the present study, Complexin-1/2/3 TKO neurons exhibited no significant changes in the number of membrane-attached synaptic vesicles in comparison to controls, correlating well to the unaltered RRP sizes measured electrophysiologically (Reim et al., 2001; Xue et al., 2007, 2008). However, recent studies described a decrease in the RRP and EPSC size and a massive increase in the mEPSC frequency after lentiviral knock-down of Complexins in mammalian mass neuron culture systems, prompting the authors to propose that Complexins function to clamp synaptic vesicles in a primed state prior to fusion, potentially by stabilizing SNARE complexes (Cao et al., 2013; Kaeser-Woo et al., 2012; Tang et al., 2006; Yang et al., 2010). This model is supported by data obtained from $C$. elegans Complexin null mutants, which revealed a severe reduction in evoked release and in the number of docked vesicles, a phenotype most likely explained by an increase in spontaneous fusion events in the absence of inhibitory clamping-functions (Hobson et al., 2011). The discrepancy between knock-down and the full knockout approaches can be explained by the introduction of a compensatory mechanism, that arises in response to long-term loss of Complexins, and ultimately reduces vesicular release probability in order to compensate for the absence of a fusionclamp and to provide a stable pool size. Alternatively, the knock-down approach could introduce false effects by inducing off-target effects of the RNAi, or a transient upregulation, or stabilization, of other molecular components of the release machinery to compensate for the decrease in neurotransmission caused by the loss of Complexins, thus explaining the increased mEPSC frequency. Moreover it has been shown that the increase in mEPSC frequency is highly $\mathrm{Ca}^{2+}$-dependent, allowing the possibility that differences between the knock-down and knockout approach might be related to differences in $\mathrm{Ca}^{2+}$-concentrations used between studies (Neher, 2010; Yang et al., 2010). It is becoming increasingly evident that different functional domains of Complexins could mediate a subtle interplay between facilitory and inhibitory effects on release (Cho et al., 
2010; Li et al., 2011; Xue et al., 2007, 2009, 2010). However, my data clearly support a facilitatory role for Complexins in neurotransmitter release, since an inhibitory clamping function would potentially result in a decrease in the number of membrane-attached synaptic vesicles as observed in C. elegans (Hobson et al., 2011).

Synaptotagmin-1 has also been proposed to function in clamping synaptic vesicles, either alone, or in concert with Complexins, in addition to its role as $\mathrm{Ca}^{2+}$-sensor for release. In mass, micronetwork or organotypic slice cultures, Synaptotagmin-1 KO neurons exhibit an increase in the mEPSC and mIPSC frequency (Kerr et al., 2008; Liu et al., 2009; Xu et al., 2009). However, in autaptic neurons, such an increase has not yet been detected (Geppert et al., 1994; Liu et al., 2009). In various experimental settings, the genetic deletion of Synaptotagmins results in small, but rarely significant, decreases in the RRP size as measured by application of hypertonic sucrose solution or by $\mathrm{Ca}^{2+}$-uncaging (Burgalossi et al., 2010; Geppert et al., 1994; Jorgensen et al., 1995; Liu et al., 2009; Tang et al., 2006; Xu et al., 2009). In the present study, a decrease in the number of membrane-attached synaptic vesicles was detected in Synaptotagmin-1 KO synapses, indicating a reduction in the RRP of fusion-competent synaptic vesicles. A decrease in the number of membrane-proximal $(0-40 \mathrm{~nm})$ synaptic vesicles was detected whereas the general distribution of synaptic vesicles in the terminal appeared unchanged. Based on these findings, it cannot be excluded that Synaptotagmins may play a regulatory role in clamping synaptic vesicles prior to release, since an increase in spontaneous release would likely be observed as a decrease in the number of docked/functionally primed synaptic vesicles at any given point in time as it has been described for C.elegans Complexin null mutants (Hobson et al., 2011). However, the molecular mechanism underlying such a clamping function has yet to be solved.

\subsection{A regulatory, but not essential role of Synaptotagmin-1 in synaptic vesicle docking/priming}

The precise molecular function of Synaptotagmin-1 prior to, or during, $\mathrm{Ca}^{2+}$-triggering has not yet been fully understood. However, two different models have recently been proposed (reviewed in Jahn and Fasshauer, 2012; Vennekate et al., 2012). Firstly, Synaptotagmin-1 could modulate synaptic vesicle priming prior to $\mathrm{Ca}^{2+}$-evoked fusion by stabilizing partially assembled SNARE complexes, thereby acting as a fusion clamp by preventing full SNARE complex assembly (See 4.8.). According to this model, Synaptotagmin-1 would respond to increases in intracellular $\mathrm{Ca}^{2+}$-concentrations by 
triggering C-terminal zippering of the SNARE complex by either (1) displacing Complexins from the partially assembled SNARE complex, (2) unclamping the SNARE complex, or (3) binding to the plasma membrane in trans, thereby destabilizing the bilayer and potentially inducing positive membrane curvature to help to overcome the energy barrier for fusion (Hui et al., 2009; Martens et al., 2007; Radhakrishnan et al., 2009). This model, which assumes at least partial SNARE complex assembly in the docked/primed synaptic vesicle state prior to synaptic vesicle fusion, is supported by the findings presented in this study. Since all three components of the neuronal SNARE complex are required for efficient synaptic vesicle docking/priming, it can be concluded that trans-SNARE interactions prior to vesicle fusion are likely to form the molecular basis of synaptic vesicle docking. In the present study, it is demonstrated that in Synaptotagmin-1 KO neurons, synaptic vesicle docking in glutamatergic synapses is only slightly reduced. Moreover, the general distribution of synaptic vesicles is unaffected by the loss of Synaptotamin-1 KO and the vast majority of the vesicles within $40 \mathrm{~nm}$ of the active zone still reside in physical contact with the plasma membrane. However, the observed decrease in the number of membrane proximal vesicle numbers appears compatible with Synaptotagmin-1 performing a clamping function. Taken together, these findings indicate that Synaptotagmin-1, though not essential for synaptic vesicle docking/priming in neurons, might perform a regulatory function in this process.

A second alternative model has recently been proposed according to which Synaptotagmin-1 performs an essential role in tethering/docking synaptic vesicles to the plasma membrane prior SNARE complex assembly (van den Bogaart et al., 2011; Jahn and Fasshauer, 2012; Vennekate et al., 2012). $\mathrm{Ca}^{2+}$-triggering by Synaptotagmin-1 would then result in $\mathrm{Ca}^{2+}$-dependent penetration of Synaptotagmin-1 into the plasma membrane, which would decrease the distance between the vesicle and plasma membrane bilayers, and would thereby bring $t$ - and v-SNAREs into interactive range for SNARE complex formation. The docked/primed state of a synaptic vesicle would be defined by $\mathrm{Ca}^{2+}$ independent Synaptotagmin-1 interactions with t-SNARE acceptor complexes and/or with $\mathrm{PIP}_{2}$ at the plasma membrane. Experimental support for this model comes mainly from in vitro studies. In the presence of $\mathrm{Ca}^{2+}$, a cytoplasmic stretch of Synaptotagmin-1 containing both $\mathrm{C}_{2}$ domains $\left(\mathrm{C}_{2} \mathrm{~A}\right.$ and $\left.\mathrm{C}_{2} \mathrm{~B}\right)$ can cluster liposomes at a distance of 3-5 nm measured after cryo-electron microscopy, which would correspond roughly to the calculated size of a single $\mathrm{Ca}^{2+}$-bound $\mathrm{C}_{2}$ domain oriented perpendicularly between the two membranes (e.g. vesicle and plasma membrane) (Araç et al., 2006; Seven et al., 2013). Recently, it was shown that $\mathrm{Ca}^{2+}$-unbound Synaptotagmin-1 could tether vesicles in a $\sim 5-28 \mathrm{~nm}$ distance from the active zone membrane and in response to elevated $\mathrm{Ca}^{2+}$ levels reduce the 
vesicle distance to below $5 \mathrm{~nm}$ by $\mathrm{Ca}^{2+}$-dependent $\mathrm{PIP}_{2}$ interactions, triggering SNARE nucleation and subsequent membrane fusion (van den Bogaart et al., 2011).

Studies assessing the role of Synaptotagmin-1 in secretory vesicle docking aimed at providing evidence for this alternative model in a more physiological environment often produced rather controversial results. In Drosophila and $C$. elegans neuromuscular junction synapses, the absence of Synaptotagmin-1 results in a dramatic reduction in the total number of synaptic vesicles and in fewer docked vesicles per terminal, whereas no effects on vesicle numbers or the RRP size were originally observed in mouse presynaptic terminals (Geppert et al., 1994; Jorgensen et al., 1995; Reist et al., 1998). Other studies, however, have reported that lack of Synaptotagmin-1 in C.elegans, Drosophila and mouse neurons causes a decrease in total presynaptic vesicle numbers in addition to a docking deficit, most likely as a result of impaired vesicle recycling (Liu et al., 2009; Reist et al., 1998; Yu et al., 2013). In the present study, a slight decrease in the synaptic vesicle terminal and cluster density in synapses was observed, a potential result of altered membrane recycling in the absence of Synaptotagmin-1. However, in mammalian hippocampal culture systems lacking Synaptotagmin-1, hypertonic sucrose solution application or $\mathrm{Ca}^{2}$-uncaging experiments revealed little, or no, change in the measured size of the RRP, therefore questioning an essential role of Synaptotagmin-1 in the synaptic vesicle priming step (Burgalossi et al., 2010; Geppert et al., 1994; Jorgensen et al., 1995; Liu et al., 2009; Xu et al., 2009). One recent study indicated a decrease in the RRP size in Synaptotagmin-1 KO dissociated hippocampal neuron cultures, which was accompanied by a decrease in the number of synaptic vesicles in presynaptic terminals and in the number of morphologically docked vesicles after high-pressure freezing, freezesubstitution and electron tomographic analysis (Liu et al., 2009).

Another study has proposed that Synaptotagmin-1 might indeed function in LDCV docking in mouse chromaffin cells (de Wit et al., 2009). Previous work by de Wit and colleagues indicated that a reduction of Syntaxin-1 protein levels by proteolytic cleavage with BoNT/C coupled with a complete genetic $\mathrm{KO}$ of the Syntaxin-1 binding partner and key regulator of secretory vesicle release, Munc18-1, results in a robust LDCV docking-defect in chemically fixed chromaffin cells with corresponding reductions in the RRP size (GulyásKovács et al., 2007; Toonen et al., 2006; Voets et al., 2001; de Wit et al., 2006, 2009). Based on these findings, de Wit and colleagues tried to solve the molecular pathway underlying LDCV docking in chromaffin cells by performing cross-rescue experiments in mouse mutants, ultimately proposing a molecular scenario for secretory vesicle docking that argues against a role of the fully assembled SNARE complex in LDCV docking (de Wit et al., 2009). In this study, de Wit and colleagues observed, in addition to the docking 
defects upon Syntaxin-1 and Munc18-1 pertubation, a deficiency in LDCV docking in mutants lacking the second t-SNARE SNAP25, for which docking deficits were not previously reported, or the $\mathrm{Ca}^{2+}$-sensor Synaptotagmin-1 (Sørensen et al., 2003; de Wit et al., 2009). The conclusion of the authors was that SNAP25/Syntaxin-1 acceptor complexes on the plasma membrane act in concert with Synaptotagmin-1 on the vesicle surface to promote LDCV docking. Since Synaptobrevin-2 KO chromaffin cells failed to reveal a decrease in the number of docked LDCVs in previous studies, de Wit and colleagues proposed that SNARE complex formation occurs downstream of LDCV membrane-attachment (Borisovska et al., 2005; Gerber et al., 2008; de Wit et al., 2009).

However, in contrast to neuronal synapses, which possess spatially and molecularly defined active zones, LDCV secretion from chromaffin cells is not restricted to specialized release sites, but rather occurs over the entire plasma membrane. As shown by de Wit and colleagues, loss of Munc18-1, Syntaxin-1, SNAP25 and Synaptotagmin-1 resulted in a loss of membrane-attached LDCVs as analyzed in two-dimensional electron micrographs after chemical and high-pressure freezing fixation (de Wit et al., 2009). However, in this study LDCVs did not only fail to dock to the plasma-membrane, but KO chromaffin cells exhibited also a change in the cumulative vesicle distribution within the first $100 \mathrm{~nm}$ of the plasma membrane, raising the possibility that the docking deficit might rather be a result of an inability of LDCVs to be transported and tethered to the plasma membrane. Moreover, a recent study using high-pressure freezing fixation for electron microscopic analysis of LDCV docking in PC12 cells revealed that proteolytic cleavage of Synaptobrevin-2 results in a decrease of membrane-attached LDCVs, with no major changes in the total number of vesicles close to the plasma membrane (Wu et al., 2012), whereas in contrast, genetic deletion of Synaptobrevin-2 in chromaffin cells has been previously shown to result in a significant increase in the number of LDCVs per chromaffin cell profile in electron micrographs and even in a slight, but not significant increase in the number of docked LDCVs (Gerber et al., 2008).

It is very likely that the discrepancy between the data for LDCV docking in chromaffin cells and synaptic vesicle docking presented here arises from differences in sample preparation and in docking definitions applied between studies. To date, none of the studies analyzing LDCV docking in chromaffin cells have employed electron tomography to resolve fine differences in membrane distances between LDCVs and the plasma membrane, but rather focused on gross changes in the vesicle distribution throughout the cell. Following our model and taking in vitro data into account, it is possible that in chromaffin cells Synaptotagmin- 1 has an important role in tethering LDCVs close to the plasma membrane (e.g. 5-28 nm) in a $\mathrm{Ca}^{2+}$-independent manner, e.g. by interactions with 
the t-SNARE acceptor complex or $\mathrm{PIP}_{2}$ in a process upstream of LDCV membraneattachment and SNARE-complex formation (van den Bogaart et al., 2011; de Wit et al., 2009). This would explain the decrease in membrane-proximal LDCVs seen in SNAP25, Syntaxin-1 and Synaptotagmin-1 KO chromaffin cells and could also explain why the loss of Synaptotagmin-1 introduces a significant decrease in the number of membrane proximal $(0-40 \mathrm{~nm})$ synaptic vesicles in our experimental setting. In a second step, downstream of LDCV tethering, LDCVs might then become physically docked/primed to the plasma membrane as a result of SNARE complex formation. Due to the technical limitations imposed by chemical fixation and conventional two-dimensional electron microscopy, past studies investigating LDCV docking in chromaffin cells have been unable to resolve small $(<5 \mathrm{~nm})$ distances between vesicle and plasma membranes. This would explain why genetic deletion of CAPS and Synaptobrevin-2 in chromaffin cells introduces major physiological deficits in the RRP size, when so far no major reductions in the number of docked LDCVs have been described (Borisovska et al., 2005; Gerber et al., 2008; Liu et al., 2008, 2010; Speidel et al., 2005). This argument is supported by the fact that proteolytic cleavage of the individual SNAREs in PC12 cells results in a docking deficit of LDCVs close to the membrane, with no changes in the LDCV distribution further away from the membrane analyzed after high-pressure freezing fixation (Wu et al. 2012). In the present study, an accumulation of LDCVs in presynaptic terminals of Synaptobrevin-2 KO synapses was detected, which was even more dramatic than that observed for SNAP25 and Syntaxin-1 deficient neurons. In these genotypes, LDCVs were able to come in close proximity to the plasma membrane $(<40 \mathrm{~nm})$, a phenomenon that was hardly ever observed in control synapses, but vesicles were never in physical plasma membrane contact.

In summary, I propose that the following processes occur sequentially prior to vesicle fusion: (1) $\mathrm{Ca}^{2}$-independent interactions between Synaptotagmin-1 and the t-SNAREs SNAP25 and Syntaxin-1, possibly in a $\mathrm{PIP}_{2}$ dependent manner, tether secretory vesicles close to the plasma membrane $(5-28 \mathrm{~nm})$. This interaction, although potentially crucial for LDCV release in chromaffin cells, may be less critical in neuronal synapses due to the dense active zone protein network, which might be able to employ alternative mechanisms to tether vesicles close to the plasma membrane. Moreover, CAPS proteins might be involved in the first tethering step since $\mathrm{KO}$ mice lack a prominent accumulation of tethered vesicles close to the plasma membrane. (2) In this membrane-proximal state of vesicles, members of the Munc13 and/or CAPS protein family can prime synaptic vesicles for fusion by initiating or accelerating SNARE complex assembly. The interactions between $t$ - and v-SNAREs in trans requires vesicles to be within $5 \mathrm{~nm}$ of the 
plasma membrane, therefore physiologically primed vesicles appear as membraneattached or docked. At this stage, Synaptotagmin-1 interactions with the SNARE complex might have a role in clamping vesicles in a release-competent state prior to fusion and Complexins might stabilize partially-assembled SNARE-complexes to secure a fast response after the arrival of an action potential. (3) $\mathrm{Ca}^{2}$-triggering would then be mediated by Synaptotgamin-1, which could act by destabilizing the membranes, by inducing membrane curvature to trigger fusion or by displacing another fusion clamp to initiate and synchronize full zippering of multiple SNARE complexes.

Essentially, the data presented in this study support a model according to which synaptic vesicles require trans-SNARE interactions for membrane-attachment. Synaptotagmin-1 might have an upstream regulatory or tethering function in this process. This model does not differ by much from the mechanism proposed by Mohrmann and Sorensen (2012), according to which Synaptotagmin-1/t-SNARE interactions "dock" synaptic vesicles to the plasma membrane and Munc13s can then "prime" vesicles to initiate SNARE complex formation. As it was demonstrated here, priming by Munc13s and CAPS proteins results in final membrane-attachment $(<5 \mathrm{~nm})$, which was termed "docking" and whose detection requires cryo-fixation and high-resolution three-dimensional electron microscopy techniques. Loss of Munc13s, SNAP25 and Synaptobrevin-2 causes a severe accumulation of vesicles close to the plasma membrane, possibly "tethered" to the release site by interactions with the active zone cytoskeleton in proximity of the membrane $(5-10 \mathrm{~nm})$. The initiation of this tethering could be performed by Synaptotagmin-1, since $\mathrm{Ca}^{2+}$-unbound Synaptotagmin-1 has been shown to tether liposomes within this range (i.e. 5-28 nm) (van den Bogaart et al., 2011). 


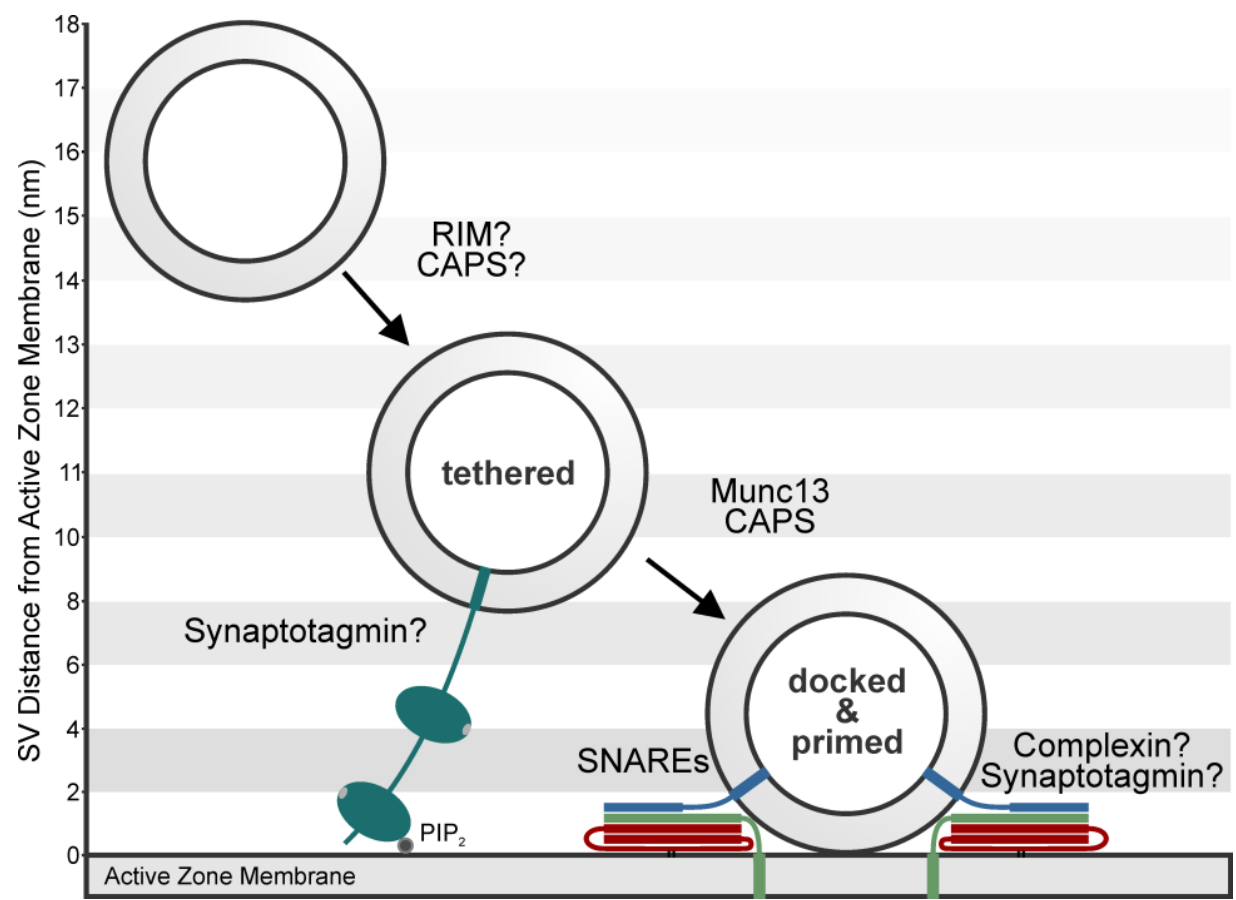

Figure 4.1. Molecular mechanisms of synaptic vesicle docking/priming

A sequential model of synaptic vesicle membrane interactions at the active zone. In wild-type synapses, RIM proteins might initiate tethering of synaptic vesicles close to the plasma membrane (Fernández-Busnadiego et al., 2013). CAPS and/or Synaptotagmin-1 (dark green) could mediate this synaptic vesicle tethering step via $\mathrm{Ca}^{2}$-independent, $\mathrm{PIP}_{2}$-dependent interactions with the plasma membrane (grey circle) and/or the t-SNARE acceptor complex (not shown). Munc13 and CAPS initiate docking/priming of synaptic vesicles in a final membrane-attachment process. The synaptic vesicle docking/ priming step depends on the individual neuronal SNARE proteins Synaptobrevin-2 (blue), Syntaxin-1 (light green), SNAP25 (red) and possibly requires at least partial SNARE complex formation. Synatptotagmins and Complexins might stabilize partiallyarrested SNARE complexes prior to fusion. Model is not drawn to scale. Molecule schematics are taken from Jahn and Fasshauer 2012.

4.10.

\section{Enlarged synaptic vesicles in Synaptobrevin-1, SNAP25 and Munc13 KO synapses}

Throughout the study, I noticed a significant increase in the vesicle diameter in synapses lacking either Munc13 proteins, or the SNARE proteins Synaptobrevin-2 or SNAP25. This increase in the vesicle size assessed by three-dimensional analysis of electron tomograms was accompanied by an accumulation of large vacuolar endosomal structures in two-dimensional electron micrographs in the aforementioned genotypes. Moreover, synapses from Syntaxin-1A / Syntaxin-1B ${ }^{\mathrm{YFP}}$ samples exhibited more endosomes per synaptic profile, despite normal synaptic vesicle sizes. The latter discrepancy is likely due to the fact that I was not investigating a full Syntaxin-1A/B DKO, but was rather studying a reduction in Syntaxin-1 levels in hypomorphic conditions. In SNAP25 and Synaptobrevin-2 
KO slices, I detected an increase in the size of VGLUT1 positive puncta, which might be associated to the respective increase in the measured synaptic vesicle volumes for these genotypes. The increase in vesicle size is unlikely to result from an increase in vesicular neurotransmitter content, since the mEPSC amplitudes were unchanged, or even slightly decreased, in Synaptobrevin-2 and SNAP25 KO mice, respectively (Bronk et al., 2007; Schoch et al., 2001). However, recent data implicating SNARE proteins in postsynaptic receptor trafficking/exocytosis emphasize the need for caution when estimating vesicular quantal sizes by analysis of mEPSCs in SNARE-deletion mutants (Jurado et al., 2013).

Indeed, it is likely that the observed increases in vesicle size are correlated to altered presynaptic membrane recycling in the absence of synaptic vesicle fusion. This coupling of exo-and endocytotic processes is thought to be largely $\mathrm{Ca}^{2+}$ and SNARE protein dependent (Deák et al., 2004; Hosoi et al., 2009; Koo et al., 2011; Peng et al., 2013; Wu et al., 2009; Xu et al., 2013). Early studies proposed that Synaptobrevin-2 could be involved in a fast endocytosis process in hippocampal synapses, since genetic deletion of Synaptobrevin-2 causes an increase in synaptic vesicle sizes (Deák et al., 2004). The observed defects in endocytosis are most likely caused by an inability of Synaptobrevin-2 to interact normally with its endocytic adaptors AP180 and clathrin assembly lymphoid myeloid leukemia (CALM). AP180 KO mice exhibited retention of Synaptobrevin-2, but not VGLUT1, in the plasma membranes and an increase in synaptic vesicle sizes comparable with that observed in Synaptobrevin-2 KO synapses in the present study (Koo et al., 2011).

SNAP25 and Syntaxin-1 were shown to interact with intersectin and dynamin, respectively, both molecules involved in synaptic membrane recycling (Galas et al., 2000; Okamoto et al., 1999). A recent study proposed a mechanism according to which the observed cell death after genetic deletion or proteolytic cleavage of Syntaxin-1 and SNAP25 is most likely caused by a defect in t-SNARE dependent membrane recycling processes rather than by the defects observed in exocytosis (Peng et al., 2013). Interestingly, a SNAP25 knock-in mouse line carrying a mutation that abolishes protein kinase C-dependent phosphorylation of SNAP25 (SNAP25 ${ }^{\text {S187A }}$ ) results in a reduction of presynaptic SNAP25 levels accompanied by a significant increase in the size of glutamatergic terminals in the striatum as assessed by VGLUT1 immunolabeling and electron microscopy (Nakata et al., 2012). Moreover, these mice commonly exhibited seizures and neurons with the knock-in mutation presented an accumulation of $\alpha$ Synuclein in presynaptic terminals, a feature associated with a range of neurodegenerative diseases ( $\alpha$-Synucleinopathies), including Parkinson's disease. Interestingly, these $\alpha$-Synuclein accumulations seem to reside at the periactive zone, a 
canonical site of endocytosis, indicating a relationship between altered endocytosis and SNAP25 expression levels in the synapse (Nakata et al. 2012). Taken together, the data presented here support the hypothesis that SNAP25 plays a role in presynaptic membrane recycling.

In the Calyx of Held synapse, a large, highly specialized synapse in the auditory pathway, exo- and endocytosis can be monitored by membrane capacitance measurements made at the presynaptic terminals (reviewed in Schneggenburger and Forsythe 2006). During high synaptic activity, e.g. upon by high-frequency stimulation, the pool of fusioncompetent vesicles has to be constantly refilled while action potential-triggered fusion remains ongoing. The rate of this constant refilling of the RRP has been shown to be diminished when endocytosis is pharmacologically blocked or when the SNARE proteins Synaptobrevin, SNAP25 and Syntaxin were proteolytically cleaved (Hosoi et al., 2009; Xu et al., 2013). One interpretation of these findings was that the rate of RRP refilling is most likely not limited by the number of presynaptic vesicles present, but rather by the speed by which key players of the molecular release machinery (i.e. SNAREs, Synaptotagmins, and vesicular neurotransmitter transporter) can be cleared from active zone release sites and/or recycled into release-competent synaptic vesicles (Hosoi et al., 2009).

The $28 \%$ increase in vesicle volume observed in Munc13-1/2 DKO samples is more difficult to explain, since so far no defects in endocytosis have been observed in these mice (Varoqueaux et al., 2002). Synapses lacking all Munc13 isoforms are completely silent, therefore exo-endocytosis coupling cannot be measured by capacitance measurements or by stimulation of FM dye uptake into vesicles. One possible explanation for the observed increase in synaptic vesicle volume could be that a constant block of neurotransmitter release prevents important vesicular proteins (i.e. Synaptotagmin-1, Synaptobrevin-2) needed for endocytosis from ever reaching the plasma membrane, a hypothesis which would be difficult to test experimentally in Munc13-1/2 DKO mice that die at birth and whose neurons are completely silent (Diril et al., 2006; Koo et al., 2011; Varoqueaux et al., 2002). However, it has been proposed that in hippocampal and Calyx of Held synapses, an increase in $\mathrm{Ca}^{2+}$-concentrations not only triggers release, but also endocytosis of a "readily-retrievable pool" of vesicles (Hua et al., 2011a; Wu et al., 2009). In the Calyx of Held, the $\mathrm{Ca}^{2+}$-dependence of endocytosis has been linked to Calmodulin, a $\mathrm{Ca}^{2+}$-dependent regulator of Munc13s (Wu et al., 2009). Indeed, Calmodulin inhibitors induce a defect in RRP refilling, which may be attributable to a slower rate of endocytosis (Sakaba and Neher, 2001; Wu et al., 2009). Interestingly, a recently published mouse line expressing a $\mathrm{Ca}^{2+}$-Calmodulin insensitive Munc13-1 variant exhibited a comparable decrease in the rate of synaptic vesicle replenishment into the RRP during high activity in 
the Calyx of Held, indicating that Munc13-1 might be the major target in $\mathrm{Ca}^{2+}$-Calmodulin dependent presynaptic plasticity processes (Lipstein et al., 2013).

The results of the present study support the possibility that Munc13s function in both synaptic vesicle priming and in coupling exo- and endocytosis at the active zone. $\mathrm{Ca}^{2+}$ Calmodulin/Munc13 interactions during high synaptic activity might then potentiate the rate of pool refilling by boosting fast endocytosis and therefore fast release machinery and membrane recycling during release. Indeed, Munc13s may be interesting candidates for such a function due to their central position at the active zone and their interactions with RIM proteins, which link Munc13s and vesicles in close proximity of $\mathrm{Ca}^{2+}$-channels (Han et al., 2011; Kaeser et al., 2011). Moreover, it has recently been shown that synaptosomes lacking RIM1a, a major Munc13-1 and ubMunc13-2 binding partner at the active zone, exhibited a $26 \%$ increase in synaptic vesicle volumes, which closely correlates with the $28 \%$ increase observed in Munc13-1/2 DKO synaptic profiles in the present study (Fernández-Busnadiego et al., 2013). RIM1a KO mice are viable, despite exhibiting defects in synaptic vesicle priming/docking, and a 60\% decrease in Munc13 levels (Andrews-Zwilling et al., 2006; Deng et al., 2011; Han et al., 2011; Schoch et al., 2002). These findings indicate that the interaction between Munc13s, RIM proteins and potentially $\mathrm{Ca}^{2+}$ - channels at the active zone might have a direct role in mediating exoand endocytosis coupling.

\subsection{A Munc13-1 conditional KO mouse line}

The aim of the second part of the present study was to generate a Munc13-1 conditional $\mathrm{KO}$ mouse line. Munc13-1 conventional KO mice exhibit severe impairments in synaptic transmission in glutamatergic synapses, causing death of the animals at birth (Augustin et al., 1999a). Within the last years it became evident that Munc13s are not only essential for priming synaptic vesicles under basal conditions, but also serve as important modulators of synaptic vesicle release through a wide range of molecular interactions with second messengers $\left(\mathrm{Ca}^{2+}, \mathrm{DAG}\right)$ in the presynaptic terminal (Lipstein et al., 2013; Rhee et al., 2002). Moreover, it has been shown that Munc13s play a crucial role in presynaptic shortand long-term plasticity processes in a variety of excitatory neuron types (e.g. Calyx of Held, hippocampal mossy fiber synapse) (Breustedt et al., 2010; Chen et al., 2013; Lipstein et al., 2013; Yang and Calakos, 2011; Zhao et al., 2012a, 2012b). The majority of these studies revealed a dominant role of Munc13-1 in the investigated neuron types, a problem that required elaborated approaches to study the role of Munc13-1 in 146 
physiological networks, e.g. in organotypic slice culture systems or by viral overexpression of dominant-negative Munc13-1 constructs in vivo (Chen et al., 2013; Yang and Calakos, 2011; Zhao et al., 2012a, 2012b). With the help of a Munc13-1 CKO mouse line, it will not only be possible to assess the consequences of Munc13-1 loss in vivo, but also to study the functions of Munc13-2 and -3 in the absence of Munc13-1. Mice carrying a genetic deletion for Munc13-2, -3 or for both of these isoforms are viable and fertile indicating that Munc13-1 can compensate for their loss and to a large extent maintain adequate synaptic transmission in the affected synapses (Augustin et al., 2001; Chen et al., 2013; Varoqueaux et al., 2002).

In the present study, I was able to generate Munc13-1 CKO mice and demonstrate that homozygously floxed animals from that line are viable and fertile. Moreover, viral expression of the Cre-recombinase protein in dissociated neurons successfully induces Cre-mediated deletion of exon 21 in the Munc13-1 gene, tested by PCR. In addition, Western blot analysis demonstrated the absence of Munc13-1 protein in Cre-recombinase expressing cultures, and of truncation products capable of exerting dominant-negative effects. Preliminary electrophysiological experiments in Cre-recombinase expressing autaptic cultures revealed significant reductions in EPSC amplitudes and RRP sizes in response to hypertonic sucrose solution by $\sim 80 \%$. To test whether Cre-mediated excision can take place in vivo, I started to cross the floxed Munc13-1 allele into a mouse line expressing Cre-recombinase under the adenoviral Ella promotor, which is expected to excise exon 21 of Munc13-1 in all neurons at an early developmental time-point (Lakso et al., 1996). Genotyping of the first offspring from these breeding pairs by PCR revealed the presence of heterozygous mice, carrying one Munc13-1 WT and one Munc13-1 Cre allele. Litters from double heterozygous breeding pairs are expected to contain mice which will carry two Munc13-1 Cre alleles and that will hopefully phenotypically resemble full Munc13-1 KO mice (Augustin et al., 1999a). Additionally, the floxed Munc13-1 allele will be crossed into a Munc13-2/3 DKO genetic background. This mouse line can be used to completely shut down synaptic transmission in a subset of neurons or at different developmental time-points, depending on the choice of promoter controlling Crerecombinase expression in specific mouse lines (Kaartinen and Nagy, 2001). Moreover, in these genetic backgrounds viral overexpression of Munc13 constructs carrying mutations in crucial regulatory domains (e.g. $\mathrm{C}_{2} \mathrm{~B}, \mathrm{C}_{1}$ ) in vivo can help to understand their respective functions in plasticity processes in defined, mature neuronal networks that have been difficult to study in culture (e.g. Calyx of Held). 


\section{Summary}

In the present study, a combination of organotypic slice culture, cryo-fixation and threedimensional electron tomographic microscopy was employed to analyse synaptic vesicle docking in the absence of key proteins mediating neurotransmitter release. In this experimental setting, loss of priming proteins from the Munc13 and CAPS family caused an almost complete absence of docked synaptic vesicles. In both cases, reductions in the number of membrane-attached synaptic vesicles correlated well with previously observed physiological deficits in synaptic vesicle priming.

These findings indicate that morphological synaptic vesicle docking and functional priming are correlates of the same molecular process. Genetic deletion, or reduced expression, of the individual SNARE proteins Synaptobrevin-2, SNAP25 and Syntaxin-1 resulted in a decreased number of membrane-attached synaptic vesicles, indicating that (partial) SNARE complex assembly underlies the molecular mechanism of synaptic vesicle docking and priming. Moreover, my data indicate that upregulation of Synaptobrevin-1 may partially compensate for the loss of Synaptobrevin-2 function in docking/priming in a subset of glutamatergic synapses. This finding supports the hypothesis that residual release observed in neurons after genetic deletion of SNARE proteins is mediated by alternative SNARE homologues. Genetic deletion of Munc13s results in an almost complete depletion of release-competent (docked/primed) synaptic vesicles, consistent with the view that Munc13s initiate synaptic vesicle docking/priming, possibly via their interaction with the t-SNARE Syntaxin-1. However, the precise stage at which SNAREcomplex assembly is arrested in Munc13-deficient synapses could not be identified in this study.

Loss of the $\mathrm{Ca}^{2+}$-sensor Synaptotagmin-1 caused a decrease in total and membraneattached synaptic vesicles. However, the reductions were not as dramatic as it would have been expected for a molecule that was formerly proposed to be the vesicular partner in vesicle docking. Based on our findings, Synaptotagmin-1 might have a role (1) in tethering synaptic vesicles to the plasma membrane prior to final membrane-attachment or (2) in clamping synaptic vesicles in a fusion-competent state until the arrival of the $\mathrm{Ca}^{2+}$-signal for triggering fusion. Genetic deletion of Complexin caused no significant changes in the number of docked synaptic vesicles, a result that provides support for a facilitatory, rather than inhibitory, role of Complexins prior to synaptic vesicle fusion.

Synapses lacking Munc13 priming proteins, or the SNARE proteins Synaptobrevin-2 or SNAP25, exhibited increased synaptic vesicle sizes, indicating that the key components of 
the synaptic release machinery might also play an important role in presynaptic membrane or protein recycling.

In a second project, I generated a conditional Munc13-1 knock-out mouse line, which will be used to study the role of Munc13-1 in defined neuronal networks in vivo. Moreover, it will allow the function of other Munc13 isoforms (bMunc13-2, ubMunc13-2 and Munc13-3) to be studied in synapses which are otherwise dominated by Munc13-1. In this study, I demonstrate that homozygously floxed mice are viable and fertile and that after lentiviral Cre-recombinase expression in dissociated hippocampal neuron cultures, Munc13-1 protein levels are undetectable by Western blot analysis. Cre-recombinase expressing autaptic neurons exhibit a massive reduction in the EPSC and RRP sizes as described for the conventional Munc13-1 knock-out mouse line (Augustin et al., 1999a). 


\section{Supplementary Figures}
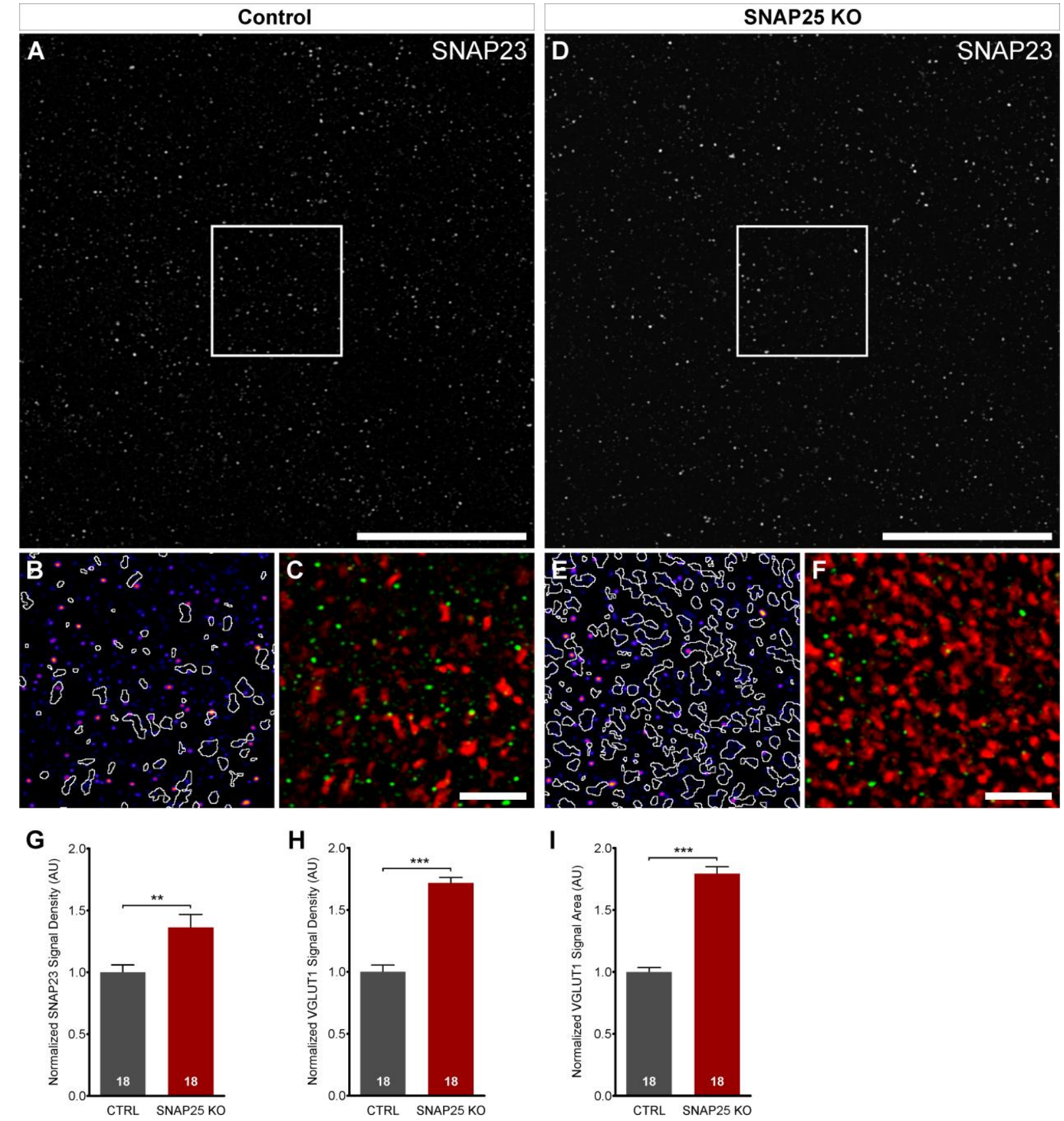

Figure S1 Light microcopic analysis of SNAP23 and VGLUT1 immunoreactivity in SNAP25 KO organotypic hippocampal slices

Confocal laser scanning micrographs reveal a punctate pattern of SNAP23 immunoreactivity in neuropil from organotypic hippocampal slices (A; white framed region enlarged in $\mathbf{B}, \mathbf{C}$ ). SNAP23 immunolabelling (shown with the 'fire' LUT to illustrate relative signal intensities) are not localized to VGLUT1-immunoreactive presynapses (white outlines; B). Merged SNAP23 (green) and VGLUT1 (red) channels (C). Data as in panels A-C, but from SNAP25 KO organotypic hippocampal slices (D-F). Normalized spatial density of punctate SNAP23 signals (G). Normalized spatial density of VGLUT1 immunoreactivity (H). Normalized mean area of VGLUT1 signals (I). G-I: Control (gray) and SNAP25 $K O\left(\right.$ red): $\mathrm{N}=2, \mathrm{n}=18 ;($ Mean $+\mathrm{SEM}), \mathrm{P}<0.001:^{* * *} ; \mathrm{P}<0.01:^{* *} ; \mathrm{P}<0.05:^{*}$. Scale bar: A,D, $10 \mu \mathrm{m} ; . \mathbf{C}, \mathbf{F}$, $2 \mu \mathrm{m}$.

Imaging and Image analysis was performed by Dr. B. Cooper. 

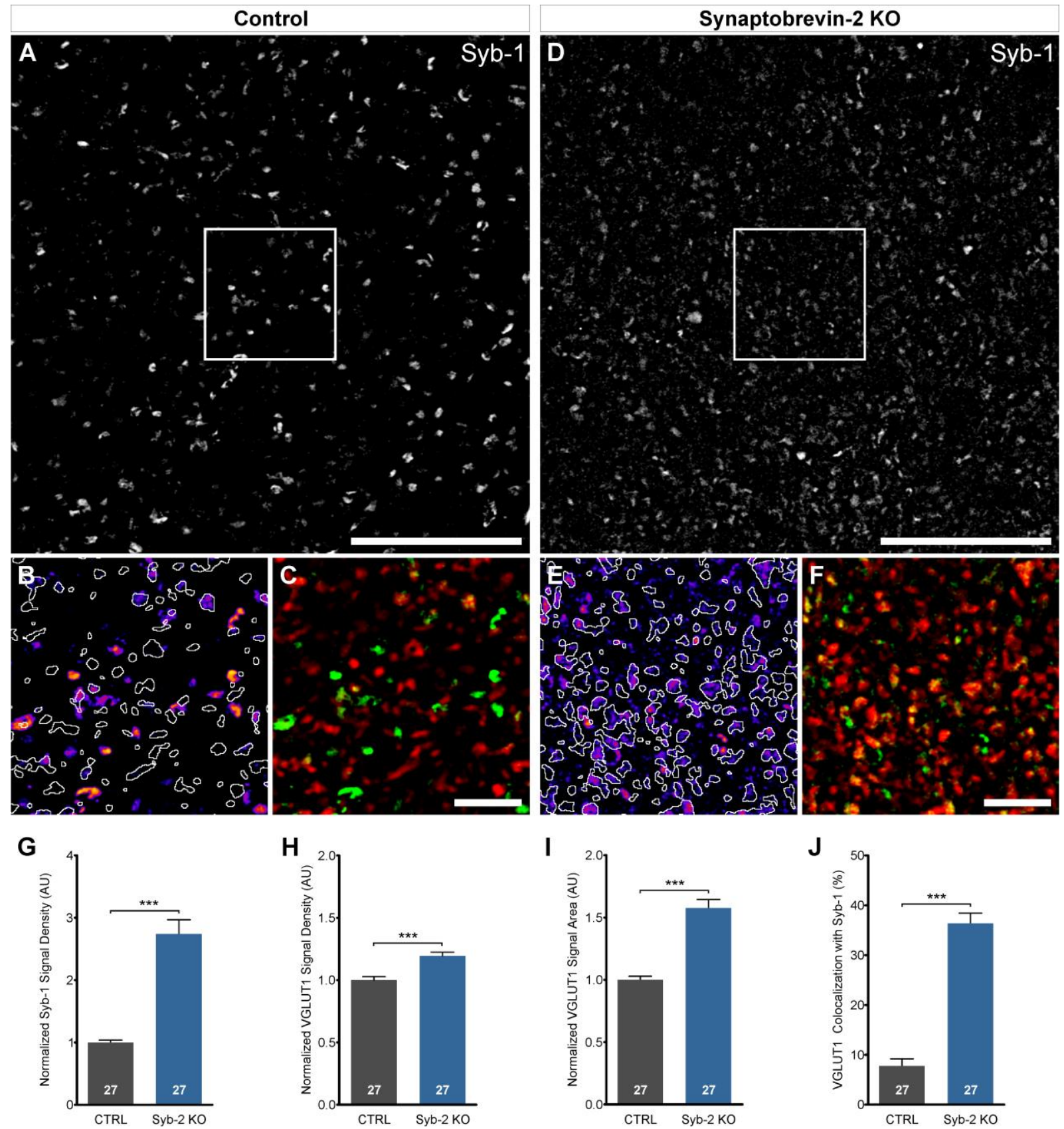

Figure S2 Light microcopic analysis of Syb-1 and VGLUT1 immunoreactivity in Syb-2 KO organotypic hippocampal slices

Confocal laser scanning micrographs of Syb-1 immunoreactivity in neuropil from organotypic hippocampal slices (A; white framed region enlarged in B, C). Syb-1 signals (shown with the 'fire' LUT to reveal relative signal intensities) are occasionally localized to VGLUT1-immunoreactive presynapses (white outlines; B). Merged Syb-1 (green) and VGLUT1 (red) channels (C). Data as in panels A-C, but from Synaptobrevin-2 (Syb-2) KO organotypic hippocampal slices (D-F). Normalized spatial density of Syb-1 signals (G). Normalized spatial density of VGLUT1 immunoreactivity $(\mathbf{H})$. Normalized mean area of VGLUT1 signals (I). The proportion of glutamatergic terminals in which Syb-1 is detected (J). G-J: Control (gray) and Syb-2 KO (blue): $\mathrm{N}=2, \mathrm{n}=27$; (Mean + SEM), $\mathrm{P}<0.001$ : ${ }^{* * *} ; \mathrm{P}<0.01$ : ${ }^{* *} ; \mathrm{P}<0.05$ : *. Scale bar: A,D, $10 \mu \mathrm{m} ;$. C,F, $2 \mu \mathrm{m}$.

Imaging and Image analysis was performed by Dr. B. Cooper. 
Adams, D.J., Quail, M.A., Cox, T., van der Weyden, L., Gorick, B.D., Su, Q., Chan, W., Davies, R., Bonfield, J.K., Law, F., et al. (2005). A genome-wide, end-sequenced 129Sv BAC library resource for targeting vector construction. Genomics $86,753-758$.

Ahmed, S., Maruyama, I.N., Kozma, R., Lee, J., Brenner, S., and Lim, L. (1992). The Caenorhabditis elegans unc-13 gene product is a phospholipid-dependent high-affinity phorbol ester receptor. Biochem. J. 287 ( Pt 3, 995-999.

Aikawa, Y., Lynch, K.L., Boswell, K.L., and Martin, T.F.J. (2006). A second SNARE role for exocytic SNAP25 in endosome fusion. Mol. Biol. Cell 17, 2113-2124.

Andrews-Zwilling, Y.S., Kawabe, H., Reim, K., Varoqueaux, F., and Brose, N. (2006). Binding to Rab3A-interacting molecule RIM regulates the presynaptic recruitment of Munc13-1 and ubMunc13-2. J. Biol. Chem. 281, 19720-19731.

Allen Developing Mouse Brain Atlas. Website: @2012 Allen Institute for Brain Science. AllenDeveloping Mouse Brain Atlas [Internet]. Available from: http://developingmouse.brain-map.org/gene/show/22074

Araç, D., Chen, X., Khant, H.A., Ubach, J., Ludtke, S.J., Kikkawa, M., Johnson, A.E., Chiu, W., Südhof, T.C., and Rizo, J. (2006). Close membrane-membrane proximity induced by $\mathrm{Ca}(2+)$-dependent multivalent binding of synaptotagmin-1 to phospholipids. Nat. Struct. Mol. Biol. 13, 209-217.

Arancillo, M., Min, S.-W., Gerber, S., Münster-Wandowski, A., Wu, Y.-J., Herman, M., Trimbuch, T., Rah, J.-C., Ahnert-Hilger, G., Riedel, D., et al. (2013). Titration of syntaxin1 in Mammalian synapses reveals multiple roles in vesicle docking, priming, and release probability. J. Neurosci. 33, 1669816714.

Aravamudan, B., Fergestad, T., Davis, W.S., Rodesch, C.K., and Broadie, K. (1999). Drosophila UNC13 is essential for synaptic transmission. Nat. Neurosci. 2, 965-971.

Aravanis, A.M., Pyle, J.L., and Tsien, R.W. (2003). Single synaptic vesicles fusing transiently and successively without loss of identity. Nature 423, 643-647.

Ashery, U., Varoqueaux, F., Voets, T., Betz, A., Thakur, P., Koch, H., Neher, E., Brose, N., and Rettig, J. (2000). Munc13-1 acts as a priming factor for large dense-core vesicles in bovine chromaffin cells. EMBO J. 19, 3586-3596.

Augustin, I., Rosenmund, C., Südhof, T.C., and Brose, N. (1999a). Munc13-1 is essential for fusion competence of glutamatergic synaptic vesicles. Nature 400, 457-461.

Augustin, I., Betz, A., Herrmann, C., Jo, T., and Brose, N. (1999b). Differential expression of two novel Munc13 proteins in rat brain. Biochem. J. 337 ( Pt 3, 363-371.

Augustin, I., Korte, S., Rickmann, M., Kretzschmar, H.A., Südhof, T.C., Herms, J.W., and Brose, N. (2001). The cerebellum-specific Munc13 isoform Munc13-3 regulates cerebellar synaptic transmission and motor learning in mice. J. Neurosci. 21, 10-17.

Basu, J., Shen, N., Dulubova, I., Lu, J., Guan, R., Guryev, O., Grishin, N. V, Rosenmund, C., and Rizo, J. (2005). A minimal domain responsible for Munc13 activity. Nat. Struct. Mol. Biol. 12, 1017-1018.

Bekkers, J.M. (1991). Excitatory and Inhibitory Autaptic Currents in Isolated Hippocampal Neurons Maintained in Cell Culture. Proc. Natl. Acad. Sci. 88, 7834-7838. 
Betz, A, Okamoto, M., Benseler, F., and Brose, N. (1997). Direct interaction of the rat unc-13 homologue Munc13-1 with the N terminus of syntaxin. J. Biol. Chem. 272, 2520-2526.

Betz, A, Thakur, P., Junge, H.J., Ashery, U., Rhee, J.S., Scheuss, V., Rosenmund, C., Rettig, J., and Brose, N. (2001). Functional interaction of the active zone proteins Munc13-1 and RIM1 in synaptic vesicle priming. Neuron 30, 183-196.

Van den Bogaart, G., Thutupalli, S., Risselada, J.H., Meyenberg, K., Holt, M., Riedel, D., Diederichsen, U., Herminghaus, S., Grubmüller, H., and Jahn, R. (2011). Synaptotagmin-1 may be a distance regulator acting upstream of SNARE nucleation. Nat. Struct. Mol. Biol. 18, 805-812.

Borisovska, M., Zhao, Y., Tsytsyura, Y., Glyvuk, N., Takamori, S., Matti, U., Rettig, J., Südhof, T., and Bruns, D. (2005). v-SNAREs control exocytosis of vesicles from priming to fusion. EMBO J. 24, 21142126.

Van de Bospoort, R., Farina, M., Schmitz, S.K., de Jong, A., de Wit, H., Verhage, M., and Toonen, R.F. (2012). Munc13 controls the location and efficiency of dense-core vesicle release in neurons. J. Cell Biol. 199, 883-891.

Bowen, M.E., Weninger, K., Brunger, A.T., and Chu, S. (2004). Single molecule observation of liposome-bilayer fusion thermally induced by soluble $\mathrm{N}$-ethyl maleimide sensitive-factor attachment protein receptors (SNAREs). Biophys. J. 87, 3569-3584.

Bradford, M.M. (1976). A rapid and sensitive method for the quantitation of microgram quantities of protein utilizing the principle of protein-dye binding. Anal. Biochem. 72, 248-254.

Brenner, S. (1974). The genetics of Caenorhabditis elegans. Genetics 77, 71-94.

Breustedt, J., Gundlfinger, A., Varoqueaux, F., Reim, K., Brose, N., and Schmitz, D. (2010). Munc13-2 differentially affects hippocampal synaptic transmission and plasticity. Cereb. Cortex 20, 1109-1120.

Broadie, K., Prokop, a, Bellen, H.J., O'Kane, C.J., Schulze, K.L., and Sweeney, S.T. (1995). Syntaxin and synaptobrevin function downstream of vesicle docking in Drosophila. Neuron 15, 663-673.

Broeke, J.H.P., Roelandse, M., Luteijn, M.J., Boiko, T., Matus, A., Toonen, R.F., and Verhage, M. (2010). Munc18 and Munc13 regulate early neurite outgrowth. Biol. Cell 102, 479-488.

Bronk, P., Deák, F., Wilson, M.C., Liu, X., Südhof, T.C., and Kavalali, E.T. (2007). Differential effects of SNAP-25 deletion on $\mathrm{Ca} 2+-$ dependent and $\mathrm{Ca} 2+$-independent neurotransmission. J. Neurophysiol. 98, 794-806.

Brose, N. (2008). For better or for worse: complexins regulate SNARE function and vesicle fusion. Traffic 9, 1403-1413.

Brose, N., Petrenko, a G., Südhof, T.C., and Jahn, R. (1992). Synaptotagmin: a calcium sensor on the synaptic vesicle surface. Science 256, 1021-1025.

Brose, N., Hofmann, K., Hata, Y., and Südhof, T.C. (1995). Mammalian homologues of Caenorhabditis elegans unc-13 gene define novel family of C2-domain proteins. J. Biol. Chem. 270, 25273-25280.

Burgalossi, A., Jung, S., Meyer, G., Jockusch, W.J., Jahn, O., Taschenberger, H., O'Connor, V.M., Nishiki, T., Takahashi, M., Brose, N., et al. (2010). SNARE protein recycling by aSNAP and BSNAP supports synaptic vesicle priming. Neuron 68, 473-487.

Burgalossi, A., Jung, S., Man, K.M., Nair, R., Jockusch, W.J., Wojcik, S.M., Brose, N., and Rhee, J.-S. (2012). Analysis of neurotransmitter release mechanisms by photolysis of caged $\mathrm{Ca}^{2+}$ in an autaptic neuron culture system. Nat. Protoc. 7, 1351-1365. 
Burnette, W.N. (1981). "Western Blotting": Electrophoretic transfer of proteins from sodium dodecyl sulfate-polyacrylamide gels to unmodified nitrocellulose and radiographic detection with antibody and radioiodinated protein A. Anal. Biochem. 112, 195-203.

Cai, H., Reim, K., Varoqueaux, F., Tapechum, S., Hill, K., Sørensen, J.B., Brose, N., and Chow, R.H. (2008). Complexin II plays a positive role in $\mathrm{Ca2}+$-triggered exocytosis by facilitating vesicle priming. Proc. Natl. Acad. Sci. U. S. A. 105, 19538-19543.

Cao, P., Yang, X., and Südhof, T.C. (2013). Complexin activates exocytosis of distinct secretory vesicles controlled by different synaptotagmins. J. Neurosci. 33, 1714-1727.

Chapman, E.R. (2008). How does synaptotagmin trigger neurotransmitter release? Annu. Rev. Biochem. 77, 615-641.

Chen, X., Tomchick, D.R., Kovrigin, E., Araç, D., Machius, M., Südhof, T.C., and Rizo, J. (2002). Threedimensional structure of the complexin/SNARE complex. Neuron 33, 397-409.

Chen, Z., Cooper, B., Kalla, S., Varoqueaux, F., and Young, S.M. (2013). The Munc13 proteins differentially regulate readily releasable pool dynamics and calcium-dependent recovery at a central synapse. J. Neurosci. 33, 8336-8351.

Cheung, G., Jupp, O.J., and Cousin, M.A. (2010). Activity-dependent bulk endocytosis and clathrindependent endocytosis replenish specific synaptic vesicle pools in central nerve terminals. J. Neurosci. 30, 8151-8161.

Cho, R., Song, Y., and Littleton, J. (2010). Comparative analysis of Drosophila and mammalian complexins as fusion clamps and facilitators of neurotransmitter release. Mol. Cell. Neurosci. 45, 389397.

Choi, U.B., Strop, P., Vrljic, M., Chu, S., Brunger, A.T., and Weninger, K.R. (2010). Single-molecule FRET-derived model of the synaptotagmin 1-SNARE fusion complex. Nat. Struct. Mol. Biol. 17, 318324.

Cooper, B., Hemmerlein, M., Ammermüller, J., Imig, C., Reim, K., Lipstein, N., Kalla, S., Kawabe, H., Brose, N., Brandstätter, J.H., et al. (2012). Munc13-independent vesicle priming at mouse photoreceptor ribbon synapses. J. Neurosci. 32, 8040-8052.

Corradini, I., Donzelli, A., Antonucci, F., Welzl, H., Loos, M., Martucci, R., De Astis, S., Pattini, L., Inverardi, F., Wolfer, D., et al. (2012). Epileptiform Activity and Cognitive Deficits in SNAP-25+/- Mice are Normalized by Antiepileptic Drugs. Cereb. Cortex bhs316-.

Cypionka, A., Stein, A., Hernandez, J.M., Hippchen, H., Jahn, R., and Walla, P.J. (2009). Discrimination between docking and fusion of liposomes reconstituted with neuronal SNARE-proteins using FCS. Proc. Natl. Acad. Sci. U. S. A. 106, 18575-18580.

Daily, N.J., Boswell, K.L., James, D.J., and Martin, T.F.J. (2010). Novel interactions of CAPS (Ca2+dependent activator protein for secretion) with the three neuronal SNARE proteins required for vesicle fusion. J. Biol. Chem. 285, 35320-35329.

Deák, F., Schoch, S., Liu, X., Südhof, T.C., and Kavalali, E.T. (2004). Synaptobrevin is essential for fast synaptic-vesicle endocytosis. Nat. Cell Biol. 6, 1102-1108.

Delgado-Martínez, I., Nehring, R.B., and Sørensen, J.B. (2007). Differential abilities of SNAP-25 homologs to support neuronal function. J. Neurosci. 27, 9380-9391.

Deng, L., Kaeser, P.S., Xu, W., and Südhof, T.C. (2011). RIM proteins activate vesicle priming by reversing autoinhibitory homodimerization of Munc13. Neuron 69, 317-331. 
Diril, M.K., Wienisch, M., Jung, N., Klingauf, J., and Haucke, V. (2006). Stonin 2 is an AP-2-dependent endocytic sorting adaptor for synaptotagmin internalization and recycling. Dev. Cell 10, 233-244.

Dittman, J., and Ryan, T.A. (2009). Molecular circuitry of endocytosis at nerve terminals. Annu. Rev. Cell Dev. Biol. 25, 133-160.

Dubochet, J. (1995). High-pressure freezing for cryoelectron microscopy. Trends Cell Biol. 5, 366-368.

Dubochet, J. 2009. "Vitreous Water." in Handbook of Cryo-preparation methods for Electon Microscopy. Taylor \& Francis Group, LLC. 2-14.

Dudenhöffer-Pfeifer, M., Schirra, C., Pattu, V., Halimani, M., Maier-Peuschel, M., Marshall, M.R., Matti, U., Becherer, U., Dirks, J., Jung, M., et al. (2013). Different Munc13 isoforms function as priming factors in lytic granule release from murine cytotoxic T lymphocytes. Traffic 14, 798-809.

Dulubova, I., Sugita, S., Hill, S., Hosaka, M., Fernandez, I., Südhof, T.C., and Rizo, J. (1999). A conformational switch in syntaxin during exocytosis: role of munc18. EMBO J. 18, 4372-4382.

Elhamdani, A., Martin, T.F., Kowalchyk, J.A., and Artalejo, C.R. (1999). Ca(2+)-dependent activator protein for secretion is critical for the fusion of dense-core vesicles with the membrane in calf adrenal chromaffin cells. J. Neurosci. 19, 7375-7383.

Farley, F.W., Soriano, P., Steffen, L.S., and Dymecki, S.M. (2000). Widespread recombinase expression using FLPeR (flipper) mice. Genesis 28, 106-110.

Fasshauer, D., and Margittai, M. (2004). A transient N-terminal interaction of SNAP-25 and syntaxin nucleates SNARE assembly. J. Biol. Chem. 279, 7613-7621.

Fasshauer, D., Sutton, R.B., Brunger, A.T., and Jahn, R. (1998). Conserved structural features of the synaptic fusion complex: SNARE proteins reclassified as Q- and R-SNAREs. Proc. Natl. Acad. Sci. 95, 15781-15786.

Feldmann, J., Callebaut, I., Raposo, G., Certain, S., Bacq, D., Dumont, C., Lambert, N., OuachéeChardin, M., Chedeville, G., Tamary, H., et al. (2003). Munc13-4 is essential for cytolytic granules fusion and is mutated in a form of familial hemophagocytic lymphohistiocytosis (FHL3). Cell 115, 461473.

Fernandez, I., Ubach, J., Dulubova, I., Zhang, X., Südhof, T.C., and Rizo, J. (1998). Three-Dimensional Structure of an Evolutionarily Conserved N-Terminal Domain of Syntaxin 1A. Cell 94, 841-849.

Fernandez, I., Araç, D., Ubach, J., Gerber, S.H., Shin, O., Gao, Y., Anderson, R.G., Südhof, T.C., and Rizo, J. (2001). Three-dimensional structure of the synaptotagmin 1 C2B-domain: synaptotagmin 1 as a phospholipid binding machine. Neuron 32, 1057-1069.

Fernández-Busnadiego, R., Zuber, B., Maurer, U.E., Cyrklaff, M., Baumeister, W., and Lucic, V. (2010). Quantitative analysis of the native presynaptic cytomatrix by cryoelectron tomography. J. Cell Biol. 188, $145-156$.

Fernández-Busnadiego, R., Schrod, N., Kochovski, Z., Asano, S., Vanhecke, D., Baumeister, W., and Lucic, V. (2011). Insights into the molecular organization of the neuron by cryo-electron tomography. J. Electron Microsc. (Tokyo). 60 Suppl 1, S137-48.

Fernández-Busnadiego, R., Asano, S., Oprisoreanu, A.-M., Sakata, E., Doengi, M., Kochovski, Z., Zürner, M., Stein, V., Schoch, S., Baumeister, W., et al. (2013). Cryo-electron tomography reveals a critical role of RIM1a in synaptic vesicle tethering. J. Cell Biol. 201, 725-740. 
Fernández-Chacón, R., Königstorfer, a, Gerber, S.H., García, J., Matos, M.F., Stevens, C.F., Brose, N., Rizo, J., Rosenmund, C., and Südhof, T.C. (2001). Synaptotagmin I functions as a calcium regulator of release probability. Nature $410,41-49$.

Frengen, E., Weichenhan, D., Zhao, B., Osoegawa, K., van Geel, M., and de Jong, P.J. (1999). A modular, positive selection bacterial artificial chromosome vector with multiple cloning sites. Genomics 58, 250-253.

Frotscher, M., Zhao, S., Graber, W., Drakew, A., and Studer, D. (2007). New ways of looking at synapses. Histochem. Cell Biol. 128, 91-96.

Gähwiler, B. (1997). Organotypic slice cultures: a technique has come of age. Trends Neurosci. 20, 471-477.

Galas, M.C., Chasserot-Golaz, S., Dirrig-Grosch, S., and Bader, M.F. (2000). Presence of dynamin-syntaxin complexes associated with secretory granules in adrenal chromaffin cells. J. Neurochem. 75, 1511-1519.

Gandhi, S.P., and Stevens, C.F. (2003). Three modes of synaptic vesicular recycling revealed by single-vesicle imaging. 423, 607-613.

Gaugler, M.N., Genc, O., Bobela, W., Mohanna, S., Ardah, M.T., El-Agnaf, O.M., Cantoni, M., Bensadoun, J.-C., Schneggenburger, R., Knott, G.W., et al. (2012). Nigrostriatal overabundance of $\alpha$ synuclein leads to decreased vesicle density and deficits in dopamine release that correlate with reduced motor activity. Acta Neuropathol. 123, 653-669.

Geppert, M., Goda, Y., Hammer, R.E., Li, C., Rosahl, T.W., Stevens, C.F., and Südhof, T.C. (1994). Synaptotagmin I: a major Ca2+ sensor for transmitter release at a central synapse. Cell 79, 717-727.

Gerber, S.H., Rah, J.-C., Min, S.-W., Liu, X., de Wit, H., Dulubova, I., Meyer, A.C., Rizo, J., Arancillo, M., Hammer, R.E., et al. (2008). Conformational switch of syntaxin-1 controls synaptic vesicle fusion. Science 321, 1507-1510.

Giddings, T.H. (2003). Freeze-substitution protocols for improved visualization of membranes in highpressure frozen samples. J. Microsc. 212, 53-61.

Gracheva, E.O., Burdina, A.O., Holgado, A.M., Berthelot-Grosjean, M., Ackley, B.D., Hadwiger, G., Nonet, M.L., Weimer, R.M., and Richmond, J.E. (2006). Tomosyn inhibits synaptic vesicle priming in Caenorhabditis elegans. PLoS Biol. 4, e261.

Gracheva, E.O., Maryon, E.B., Berthelot-Grosjean, M., and Richmond, J.E. (2010). Differential Regulation of Synaptic Vesicle Tethering and Docking by UNC-18 and TOM-1. Front. Synaptic Neurosci. 2, 141.

Granseth, B., Odermatt, B., Royle, S.J., and Lagnado, L. (2006). Clathrin-mediated endocytosis is the dominant mechanism of vesicle retrieval at hippocampal synapses. Neuron $51,773-786$.

Granseth, B., Odermatt, B., Royle, S.J., and Lagnado, L. (2009). Comment on "The dynamic control of kiss-and-run and vesicular reuse probed with single nanoparticles". Science 325, 1499; author reply 1499.

Gray, E.G. (1959). Axo-somatic and axo-dendritic synapses of the cerebral cortex: an electron microscope study. J. Anat. 93, 420-433.

Gray, E.G. (1963). Electron microscopy of presynaptic organelles of the spinal cord. J. Anat. 97, 101106. 
Grishanin, R.N., Klenchin, V.A., Loyet, K.M., Kowalchyk, J.A., Ann, K., and Martin, T.F.J. (2002). Membrane association domains in $\mathrm{Ca} 2+-$ dependent activator protein for secretion mediate plasma membrane and dense-core vesicle binding required for Ca2+-dependent exocytosis. J. Biol. Chem. 277, 22025-22034.

Grishanin, R.N., Kowalchyk, J.A., Klenchin, V.A., Ann, K., Earles, C.A., Chapman, E.R., Gerona, R.R.L., and Martin, T.F.J. (2004). CAPS acts at a prefusion step in dense-core vesicle exocytosis as a PIP2 binding protein. Neuron 43, 551-562.

Gulyás-Kovács, A., de Wit, H., Milosevic, I., Kochubey, O., Toonen, R., Klingauf, J., Verhage, M., and Sørensen, J.B. (2007). Munc18-1: sequential interactions with the fusion machinery stimulate vesicle docking and priming. J. Neurosci. 27, 8676-8686.

Hammarlund, M., Palfreyman, M.T., Watanabe, S., Olsen, S., and Jorgensen, E.M. (2007). Open syntaxin docks synaptic vesicles. PLoS Biol. 5, e198.

Hammarlund, M., Watanabe, S., Schuske, K., and Jorgensen, E.M. (2008). CAPS and syntaxin dock dense core vesicles to the plasma membrane in neurons. J. Cell Biol. 180, 483-491.

Han, Y., Kaeser, P.S., Südhof, T.C., and Schneggenburger, R. (2011). RIM determines $\mathrm{Ca}^{2}+$ channel density and vesicle docking at the presynaptic active zone. Neuron 69, 304-316.

Hayat, M.A.. 2000. "Principles and Techniques of Electron Microscopy - Biological applications." Cambridge University Press 4th ed.

Heeroma, J.H., Roelandse, M., Wierda, K., van Aerde, K.I., Toonen, R.F.G., Hensbroek, R. a, Brussaard, A., Matus, A., and Verhage, M. (2004). Trophic support delays but does not prevent cellintrinsic degeneration of neurons deficient for munc18-1. Eur. J. Neurosci. 20, 623-634.

Hernandez, J.M., Stein, A., Behrmann, E., Riedel, D., Cypionka, A., Farsi, Z., Walla, P.J., Raunser, S., and Jahn, R. (2012). Membrane fusion intermediates via directional and full assembly of the SNARE complex. Science 336, 1581-1584.

Heuser, J.E., and Reese, T.S. (1973). Evidence for recycling of synaptic vesicle membrane during transmitter release at the frog neuromuscular junction. J. Cell Biol. 57, 315-344.

Heuser, J.E., Reese, T.S., Dennis, M.J., Jan, Y., Jan, L., and Evans, L. (1979). Synaptic vesicle exocytosis captured by quick freezing and correlated with quantal transmitter release. J. Cell Biol. 81, 275-300.

Hobson, R.J., Liu, Q., Watanabe, S., and Jorgensen, E.M. (2011). Complexin maintains vesicles in the primed state in C. elegans. Curr. Biol. 21, 106-113.

Holmes, D.S., and Quigley, M. (1981). A rapid boiling method for the preparation of bacterial plasmids. Anal. Biochem. 114, 193-197.

Holt, M., Varoqueaux, F., Wiederhold, K., Takamori, S., Urlaub, H., Fasshauer, D., and Jahn, R. (2006). Identification of SNAP-47, a novel Qbc-SNARE with ubiquitous expression. J. Biol. Chem. 281, 1707617083.

Hong, W. (2005). SNAREs and traffic. Biochim. Biophys. Acta 1744, 120-144.

Honigmann, A., van den Bogaart, G., Iraheta, E., Risselada, H.J., Milovanovic, D., Mueller, V., Müllar, S., Diederichsen, U., Fasshauer, D., Grubmüller, H., et al. (2013). Phosphatidylinositol 4,5bisphosphate clusters act as molecular beacons for vesicle recruitment. Nat. Struct. Mol. Biol. 20,679686. 
Hosoi, N., Holt, M., and Sakaba, T. (2009). Calcium dependence of exo- and endocytotic coupling at a glutamatergic synapse. Neuron 63, 216-229.

Hu, Z., Tong, X.-J., and Kaplan, J.M. (2013). UNC-13L, UNC-13S, and Tomosyn form a protein code for fast and slow neurotransmitter release in Caenorhabditis elegans. Elife 2, e00967-e00967.

Hua, Y., Sinha, R., Thiel, C.S., Schmidt, R., Hüve, J., Martens, H., Hell, S.W., Egner, A., and Klingauf, J. (2011a). A readily retrievable pool of synaptic vesicles. Nat. Neurosci. 14, 833-839.

Hua, Z., Leal-Ortiz, S., Foss, S.M., Waites, C.L., Garner, C.C., Voglmaier, S.M., and Edwards, R.H. (2011b). v-SNARE composition distinguishes synaptic vesicle pools. Neuron 71, 474-487.

Hui, E., Johnson, C.P., Yao, J., Dunning, F.M., and Chapman, E.R. (2009). Synaptotagmin-mediated bending of the target membrane is a critical step in $\mathrm{Ca}(2+)$-regulated fusion. Cell $138,709-721$.

Hunt, J.M., Bommert, K., Charlton, M.P., Kistner, a, Habermann, E., Augustine, G.J., and Betz, H. (1994). A post-docking role for synaptobrevin in synaptic vesicle fusion. Neuron 12, 1269-1279.

Jahn, R., and Fasshauer, D. (2012). Molecular machines governing exocytosis of synaptic vesicles. Nature 490, 201-207.

Jahn, R., and Scheller, R.H. (2006). SNAREs - engines for membrane fusion. Nat. Rev. - Mol. Cell Biol. 7, 631-643.

James, D.J., Khodthong, C., Kowalchyk, J.A., and Martin, T.F.J. (2008). Phosphatidylinositol 4,5bisphosphate regulates SNARE-dependent membrane fusion. J. Cell Biol. 182, 355-366.

James, D.J., Kowalchyk, J., Daily, N., Petrie, M., and Martin, T.F.J. (2009). CAPS drives trans-SNARE complex formation and membrane fusion through syntaxin interactions. Proc. Natl. Acad. Sci. U. S. A. 106, 17308-17313.

James, D.J., Khodthong, C., Kowalchyk, J.A., and Martin, T.F.J. (2010). Phosphatidylinositol 4,5bisphosphate regulation of SNARE function in membrane fusion mediated by CAPS. Adv. Enzyme Regul. 50,62-70.

Jiménez, N., Vocking, K., van Donselaar, E.G., Humbel, B.M., Post, J.A., and Verkleij, A.J. (2009). Tannic acid-mediated osmium impregnation after freeze-substitution: a strategy to enhance membrane contrast for electron tomography. J. Struct. Biol. 166, 103-106.

Jockusch, W.J., Speidel, D., Sigler, A., Sørensen, J.B., Varoqueaux, F., Rhee, J.-S., and Brose, N. (2007). CAPS-1 and CAPS-2 are essential synaptic vesicle priming proteins. Cell 131, 796-808.

Jorgensen, E., Hartwieg, E., and Schuske, K. (1995). Defective recycling of synaptic vesicles in synaptotagmin mutants of Caenorhabditis elegans. Nature 378, 196-199.

Jorquera, R.A., Huntwork-Rodriguez, S., Akbergenova, Y., Cho, R.W., and Littleton, J.T. (2012). Complexin controls spontaneous and evoked neurotransmitter release by regulating the timing and properties of synaptotagmin activity. J. Neurosci. 32, 18234-18245.

Junge, H.J., Rhee, J.-S., Jahn, O., Varoqueaux, F., Spiess, J., Waxham, M.N., Rosenmund, C., and Brose, N. (2004). Calmodulin and Munc13 form a Ca2+ sensor/effector complex that controls shortterm synaptic plasticity. Cell 118, 389-401.

Jurado, S., Goswami, D., Zhang, Y., Molina, A.J.M., Südhof, T.C., and Malenka, R.C. (2013). LTP requires a unique postsynaptic SNARE fusion machinery. Neuron $77,542-558$. 
Kaartinen, V., and Nagy, A. (2001). Removal of the floxed neo gene from a conditional knockout allele by the adenoviral Cre recombinase in vivo. Genesis 31, 126-129.

Kaeser, P.S., Deng, L., Chávez, A.E., Liu, X., Castillo, P.E., and Südhof, T.C. (2009). ELKS2alpha/CAST deletion selectively increases neurotransmitter release at inhibitory synapses. Neuron 64, 227-239.

Kaeser, P.S., Deng, L., Wang, Y., Dulubova, I., Liu, X., Rizo, J., and Südhof, T.C. (2011). RIM proteins tether Ca2+ channels to presynaptic active zones via a direct PDZ-domain interaction. Cell 144, 282 295.

Kaeser, P.S., Deng, L., Fan, M., and Südhof, T.C. (2012). RIM genes differentially contribute to organizing presynaptic release sites. Proc. Natl. Acad. Sci. U. S. A. 109, 11830-11835.

Kaeser-Woo, Y.J., Yang, X., and Südhof, T.C. (2012). C-terminal complexin sequence is selectively required for clamping and priming but not for $\mathrm{Ca} 2+$ triggering of synaptic exocytosis. J. Neurosci. 32, 2877-2885.

Kasai, H., Takahashi, N., and Tokumaru, H. (2012). Distinct initial SNARE configurations underlying the diversity of exocytosis. Physiol. Rev. 92, 1915-1964.

Kerr, A.M., Reisinger, E., and Jonas, P. (2008). Differential dependence of phasic transmitter release on synaptotagmin 1 at GABAergic and glutamatergic hippocampal synapses. Proc. Natl. Acad. Sci. U. S. A. $105,15581-15586$.

Khodthong, C., Kabachinski, G., James, D.J., and Martin, T.F.J. (2011). Munc13 homology domain-1 in CAPS/UNC31 mediates SNARE binding required for priming vesicle exocytosis. Cell Metab. 14, 254263.

Kim, J.-Y., Choi, B.-K., Choi, M.-G., Kim, S.-A., Lai, Y., Shin, Y.-K., and Lee, N.K. (2012). Solution single-vesicle assay reveals PIP2-mediated sequential actions of synaptotagmin-1 on SNAREs. EMBO J. 31, 2144-2155.

Kloepper, T.H., Kienle, C.N., and Fasshauer, D. (2007). An elaborate classification of SNARE proteins sheds light on the conservation of the eukaryotic endomembrane system. Mol. Biol. Cell 18, 34633471.

Kloepper, T.H., Kienle, C.N., and Fasshauer, D. (2008). SNAREing the basis of multicellularity: consequences of protein family expansion during evolution. Mol. Biol. Evol. 25, 2055-2068.

Koch, H., Hofmann, K., and Brose, N. (2000). Definition of Munc13-homology-domains and characterization of a novel ubiquitously expressed Munc13 isoform. Biochem. J. 349, 247-253.

Kohn, R.E., Duerr, J.S., McManus, J.R., Duke, A., Rakow, T.L., Maruyama, H., Moulder, G., Maruyama, I.N., Barstead, R.J., and Rand, J.B. (2000). Expression of multiple UNC-13 proteins in the Caenorhabditis elegans nervous system. Mol. Biol. Cell 11, 3441-3452.

Kononenko, N.L., Diril, M.K., Puchkov, D., Kintscher, M., Koo, S.J., Pfuhl, G., Winter, Y., Wienisch, M., Klingauf, J., Breustedt, J., et al. (2013). Compromised fidelity of endocytic synaptic vesicle protein sorting in the absence of stonin 2. Proc. Natl. Acad. Sci. U. S. A. 110, E526-35.

Koo, S.J., Markovic, S., Puchkov, D., Mahrenholz, C.C., Beceren-Braun, F., Maritzen, T., Dernedde, J., Volkmer, R., Oschkinat, H., and Haucke, V. (2011). SNARE motif-mediated sorting of synaptobrevin by the endocytic adaptors clathrin assembly lymphoid myeloid leukemia (CALM) and AP180 at synapses. Proc. Natl. Acad. Sci. U. S. A. 108, 13540-13545.

Koster, a J., Grimm, R., Typke, D., Hegerl, R., Stoschek, a, Walz, J., and Baumeister, W. (1997). Perspectives of molecular and cellular electron tomography. J. Struct. Biol. 120, 276-308. 
Kremer, J.R., Mastronarde, D.N., and McIntosh, J.R. (1996). Computer visualization of threedimensional image data using IMOD. J. Struct. Biol. 116, 71-76.

Kyoung, M., Srivastava, A., Zhang, Y., Diao, J., Vrljic, M., Grob, P., Nogales, E., Chu, S., and Brunger, A.T. (2011). In vitro system capable of differentiating fast $\mathrm{Ca2+-triggered} \mathrm{content} \mathrm{mixing} \mathrm{from} \mathrm{lipid}$ exchange for mechanistic studies of neurotransmitter release. Proc. Natl. Acad. Sci. U. S. A. 108, E304-13.

Lackner, M. (1999). Facilitation of Synaptic Transmission by EGL-30 Gq? and EGL-8 PLC?DAG Binding to UNC-13 Is Required to Stimulate Acetylcholine Release. Neuron 24, 335-346.

Laemmli, U.K. (1970). Cleavage of Structural Proteins during the Assembly of the Head of Bacteriophage T4. Nature 227, 680-685.

Lai, A.L., Huang, H., Herrick, D.Z., Epp, N., and Cafiso, D.S. (2011). Synaptotagmin 1 and SNAREs form a complex that is structurally heterogeneous. J. Mol. Biol. 405, 696-706.

Lakso, M., Pichel, J.G., Gorman, J.R., Sauer, B., Okamoto, Y., Lee, E., Alt, F.W., and Westphal, H. (1996). Efficient in vivo manipulation of mouse genomic sequences at the zygote stage. Proc. Natl. Acad. Sci. U. S. A. 93, 5860-5865.

Landis, D.M., Hall, a K., Weinstein, L. a, and Reese, T.S. (1988). The organization of cytoplasm at the presynaptic active zone of a central nervous system synapse. Neuron 1, 201-209.

Lee, E.C., Yu, D., Martinez de Velasco, J., Tessarollo, L., Swing, D.A., Court, D.L., Jenkins, N.A., and Copeland, N.G. (2001). A highly efficient Escherichia coli-based chromosome engineering system adapted for recombinogenic targeting and subcloning of BAC DNA. Genomics 73, 56-65.

Li, F., Pincet, F., Perez, E., Eng, W.S., Melia, T.J., Rothman, J.E., and Tareste, D. (2007). Energetics and dynamics of SNAREpin folding across lipid bilayers. Nat. Struct. Mol. Biol. 14, 890-896.

Li, F., Pincet, F., Perez, E., Giraudo, C.G., Tareste, D., and Rothman, J.E. (2011). Complexin activates and clamps SNAREpins by a common mechanism involving an intermediate energetic state. Nat. Struct. Mol. Biol. 18, 941-946.

Li, L., Shin, O.-H., Rhee, J.-S., Araç, D., Rah, J.-C., Rizo, J., Südhof, T., and Rosenmund, C. (2006). Phosphatidylinositol phosphates as co-activators of $\mathrm{Ca} 2+$ binding to $\mathrm{C} 2$ domains of synaptotagmin 1 . J. Biol. Chem. 281, 15845-15852.

Lipstein, N., Schaks, S., Dimova, K., Kalkhof, S., Ihling, C., Kölbel, K., Ashery, U., Rhee, J., Brose, N., $\mathrm{Sinz}$, A., et al. (2012). Nonconserved $\mathrm{Ca}(2+) /$ calmodulin binding sites in Munc13s differentially control synaptic short-term plasticity. Mol. Cell. Biol. 32, 4628-4641.

Lipstein, N., Sakaba, T., Cooper, B.H., Lin, K.-H., Strenzke, N., Ashery, U., Rhee, J.-S., Taschenberger, H., Neher, E., and Brose, N. (2013). Dynamic control of synaptic vesicle replenishment and short-term plasticity by ca(2+)-calmodulin-munc13-1 signaling. Neuron 79, 82-96.

Liu, H., Dean, C., Arthur, C.P., Dong, M., and Chapman, E.R. (2009). Autapses and networks of hippocampal neurons exhibit distinct synaptic transmission phenotypes in the absence of synaptotagmin I. J. Neurosci. 29, 7395-7403.

Liu, H., Chapman, E.R., and Dean, C. (2013). "Self" versus "non-self" connectivity dictates properties of synaptic transmission and plasticity. PLoS One 8 , e62414.

Liu, P., Jenkins, N. a, and Copeland, N.G. (2003). A highly efficient recombineering-based method for generating conditional knockout mutations. Genome Res. 13, 476-484. 
Liu, Y., Schirra, C., Stevens, D.R., Matti, U., Speidel, D., Hof, D., Bruns, D., Brose, N., and Rettig, J. (2008). CAPS facilitates filling of the rapidly releasable pool of large dense-core vesicles. J. Neurosci. $28,5594-5601$.

Liu, Y., Schirra, C., Edelmann, L., Matti, U., Rhee, J., Hof, D., Bruns, D., Brose, N., Rieger, H., Stevens, D.R., et al. (2010a). Two distinct secretory vesicle-priming steps in adrenal chromaffin cells. J. Cell Biol. 190, 1067-1077.

Liu, Y., Schirra, C., Edelmann, L., Matti, U., Rhee, J., Hof, D., Bruns, D., Brose, N., Rieger, H., Stevens, D.R., et al. (2010b). Two distinct secretory vesicle-priming steps in adrenal chromaffin cells. J. Cell Biol. 190, 1067-1077.

Lois, C., Hong, E.J., Pease, S., Brown, E.J., and Baltimore, D. (2002). Germline transmission and tissue-specific expression of transgenes delivered by lentiviral vectors. Science 295, 868-872.

Ma, C., Li, W., Xu, Y., and Rizo, J. (2011). Munc13 mediates the transition from the closed syntaxinMunc18 complex to the SNARE complex. Nat. Struct. Mol. Biol. 18, 542-549.

Ma, C., Su, L., Seven, A.B., Xu, Y., and Rizo, J. (2013). Reconstitution of the vital functions of Munc18 and Munc13 in neurotransmitter release. Science 339, 421-425.

Maritzen, T., Podufall, J., and Haucke, V. (2010). Stonins--specialized adaptors for synaptic vesicle recycling and beyond? Traffic 11, 8-15.

Martens, S., Kozlov, M.M., and McMahon, H.T. (2007). How synaptotagmin promotes membrane fusion. Science 316, 1205-1208.

Maruyama, I.N., and Brenner, S. (1991). A phorbol ester/diacylglycerol-binding protein encoded by the unc-13 gene of Caenorhabditis elegans. Proc. Natl. Acad. Sci. U. S. A. 88, 5729-5733.

Mastronarde, D.N. (1997). Dual-axis tomography: an approach with alignment methods that preserve resolution. J. Struct. Biol. 120, 343-352.

Mastronarde, D.N. (2005). Automated electron microscope tomography using robust prediction of specimen movements. J. Struct. Biol. 152, 36-51.

McDonald, K. (2007). Cryopreparation methods for electron microscopy of selected model systems. Methods Cell Biol. 79, 23-56.

Megías, M., Emri, Z., Freund, T.F., and Gulyás, A.I. (2001). Total number and distribution of inhibitory and excitatory synapses on hippocampal CA1 pyramidal cells. Neuroscience 102, 527-540.

Möbius, W., Cooper, B., Kaufmann, W.A., Imig, C., Ruhwedel, T., and Snaidero, N. (2010). Electron Microscopy of the Mouse Central Nervous System. In Methods in Cell Biology Vol. 96,.

Mohrmann, R., and Sørensen, J.B. (2012). SNARE requirements en route to exocytosis: from many to few. J. Mol. Neurosci. 48, 387-394.

Mungenast, A.E. (2011). Diacylglycerol signaling underlies astrocytic ATP release. Neural Plast. 2011, 537659 .

Murk, J.L.A.N., Posthuma, G., Koster, A.J., Geuze, H.J., Verkleij, A.J., Kleijmeer, M.J., and Humbel, B.M. (2003). Influence of aldehyde fixation on the morphology of endosomes and lysosomes: quantitative analysis and electron tomography. J. Microsc. 212, 81-90.

Murthy, V.N., and Stevens, C.F. (1999). Reversal of synaptic vesicle docking at central synapses. Nat. Neurosci. 2, 503-507. 
Murthy, V.N., Sejnowski, T.J., and Stevens, C.F. (1997). Heterogeneous Release Properties of Visualized Individual Hippocampal Synapses. Neuron 18, 599-612.

Murthy, V.N., Schikorski, T., Stevens, C.F., and Zhu, Y. (2001). Inactivity Produces Increases in Neurotransmitter Release and Synapse Size. Neuron 32, 673-682.

Muzerelle, A., Alberts, P., Martinez-Arca, S., Jeannequin, O., Lafaye, P., Mazié, J.-C., Galli, T., and Gaspar, P. (2003). Tetanus neurotoxin-insensitive vesicle-associated membrane protein localizes to a presynaptic membrane compartment in selected terminal subsets of the rat brain. Neuroscience 122, 59-75.

Nakata, Y., Yasuda, T., Fukaya, M., Yamamori, S., Itakura, M., Nihira, T., Hayakawa, H., Kawanami, A., Kataoka, M., Nagai, M., et al. (2012). Accumulation of a-synuclein triggered by presynaptic dysfunction. J. Neurosci. 32, 17186-17196.

Neher, E. (2010). Complexin: does it deserve its name? Neuron 68, 803-806.

Neher, E., and Sakmann, B. (1976). Single-channel currents recorded from membrane of denervated frog muscle fibres. Nature 260, 799-802.

Okamoto, M., Schoch, S., and Südhof, T.C. (1999). EHSH1/intersectin, a protein that contains EH and $\mathrm{SH} 3$ domains and binds to dynamin and SNAP-25. A protein connection between exocytosis and endocytosis? J. Biol. Chem. 274, 18446-18454.

Pan, P.-Y., Cai, Q., Lin, L., Lu, P.-H., Duan, S., and Sheng, Z.-H. (2005). SNAP-29-mediated modulation of synaptic transmission in cultured hippocampal neurons. J. Biol. Chem. 280, 2576925779 .

Parsaud, L., Li, L., Jung, C.H., Park, S., Saw, N.M.N., Park, S., Kim, M.Y., and Sugita, S. (2013). Calcium-dependent Activator Protein for Secretion 1 (CAPS1) Binds to Syntaxin-1 in a Distinct Mode from Munc13-1. J. Biol. Chem. 288, 23050-23063.

Peng, L., Liu, H., Ruan, H., Tepp, W.H., Stoothoff, W.H., Brown, R.H., Johnson, E.A., Yao, W.-D., Zhang, S.-C., and Dong, M. (2013). Cytotoxicity of botulinum neurotoxins reveals a direct role of syntaxin 1 and SNAP-25 in neuron survival. Nat. Commun. 4, 1472.

Pevsner, J., Hsu, S.-C., Braun, J.E.A., Calakos, N., Ting, A.E., Bennett, M.K., and Scheller, R.H. (1994). Specificity and regulation of a synaptic vesicle docking complex. Neuron 13, 353-361.

Pfenninger, K., Sandri, C., Akert, K., and Eugster, C.H. (1969). Contribution to the problem of structural organization of the presynaptic area. Brain Res. 12, 10-18.

Pfenninger, K., Akert, K., Moor, H., and Sandri, C. (1972). The fine structure of freeze-fractured presynaptic membranes. J. Neurocytol. 1, 129-149.

Pobbati, A. V, Stein, A., and Fasshauer, D. (2006). N- to C-terminal SNARE complex assembly promotes rapid membrane fusion. Science 313, 673-676.

Poirier, M.A., Xiao, W., Macosko, J.C., Chan, C., Shin, Y.K., and Bennett, M.K. (1998). The synaptic SNARE complex is a parallel four-stranded helical bundle. Nat. Struct. Biol. 5, 765-769.

Pyott, S.J., and Rosenmund, C. (2002). The effects of temperature on vesicular supply and release in autaptic cultures of rat and mouse hippocampal neurons. J. Physiol. 539, 523-535.

Radhakrishnan, A., Stein, A., Jahn, R., and Fasshauer, D. (2009). The Ca2+ affinity of synaptotagmin 1 is markedly increased by a specific interaction of its C2B domain with phosphatidylinositol 4,5bisphosphate. J. Biol. Chem. 284, 25749-25760. 
Reim, K., Mansour, M., Varoqueaux, F., McMahon, H.T., Südhof, T.C., Brose, N., and Rosenmund, C. (2001). Complexins Regulate a Late Step in Ca2+-Dependent Neurotransmitter Release. Cell 104, $71-$ 81.

Reim, K., Wegmeyer, H., Brandstätter, J.H., Xue, M., Rosenmund, C., Dresbach, T., Hofmann, K., and Brose, N. (2005). Structurally and functionally unique complexins at retinal ribbon synapses. J. Cell Biol. 169, 669-680.

Reim, K., Regus-Leidig, H., Ammermüller, J., El-Kordi, A., Radyushkin, K., Ehrenreich, H., Brandstätter, J.H., and Brose, N. (2009). Aberrant function and structure of retinal ribbon synapses in the absence of complexin 3 and complexin 4. J. Cell Sci. 122, 1352-1361.

Reist, N.E., Buchanan, J., Li, J., DiAntonio, a, Buxton, E.M., and Schwarz, T.L. (1998). Morphologically docked synaptic vesicles are reduced in synaptotagmin mutants of Drosophila. J. Neurosci. 18, 76627673.

Renden, R., Berwin, B., Davis, W., Ann, K., Chin, C.T., Kreber, R., Ganetzky, B., Martin, T.F., and Broadie, K. (2001). Drosophila CAPS is an essential gene that regulates dense-core vesicle release and synaptic vesicle fusion. Neuron 31, 421-437.

Rhee, J.S., Betz, A., Pyott, S., Reim, K., Varoqueaux, F., Augustin, I., Hesse, D., Südhof, T.C., Takahashi, M., Rosenmund, C., et al. (2002). Beta phorbol ester- and diacylglycerol-induced augmentation of transmitter release is mediated by Munc13s and not by PKCs. Cell 108, 121-133.

Richmond, J.E., Davis, W.S., and Jorgensen, E.M. (1999). UNC-13 is required for synaptic vesicle fusion in C. elegans. Nat. Neurosci. 2, 959-964.

Richmond, J.E., Weimer, R.M., and Jorgensen, E.M. (2001). An open form of syntaxin bypasses the requirement for UNC-13 in vesicle priming. Nature 412, 338-341.

Rickman, C., Archer, D.A., Meunier, F.A., Craxton, M., Fukuda, M., Burgoyne, R.D., and Davletov, B. (2004). Synaptotagmin interaction with the syntaxin/SNAP-25 dimer is mediated by an evolutionarily conserved motif and is sensitive to inositol hexakisphosphate. J. Biol. Chem. 279, 12574-12579.

Rizo, J., and Südhof, T.C. (2012). The membrane fusion enigma: SNAREs, Sec1/Munc18 proteins, and their accomplices--guilty as charged? Annu. Rev. Cell Dev. Biol. 28, 279-308.

Rizo, J., Chen, X., and Araç, D. (2006). Unraveling the mechanisms of synaptotagmin and SNARE function in neurotransmitter release. Trends Cell Biol. 16, 339-350.

Rodríguez-Castañeda, F., Maestre-Martínez, M., Coudevylle, N., Dimova, K., Junge, H., Lipstein, N., Lee, D., Becker, S., Brose, N., Jahn, O., et al. (2010). Modular architecture of Munc13/calmodulin complexes: dual regulation by $\mathrm{Ca} 2+$ and possible function in short-term synaptic plasticity. EMBO J.29, 680-691.

Rosenmund, C., and Stevens, C.F. (1996). Definition of the Readily Releasable Pool of Vesicles at Hippocampal Synapses. Neuron 16, 1197-1207.

Rostaing, P., Real, E., Siksou, L., Lechaire, J., Boudier, T., Boeckers, T.M., Gertler, F., Gundelfinger, E.D., Triller, A., and Marty, S. (2006). Analysis of synaptic ultrastructure without fixative using highpressure freezing and tomography. Eur. J. Neurosci. 24, 3463-3474.

Rupnik, M., Kreft, M., Sikdar, S.K., Grilc, S., Romih, R., Zupancic, G., Martin, T.F., and Zorec, R. (2000). Rapid regulated dense-core vesicle exocytosis requires the CAPS protein. Proc. Natl. Acad. Sci. U. S. A. 97, 5627-5632. 
Sabatini, D.D., Bensch, K., and Barrnett, R.J. (1963). Cytochemistry and electron microscopy. The preservation of cellular ultrastructure and enzymatic activity by aldehyde fixation. J. Cell Biol. 17, 1958.

Sadakata, T., Itakura, M., Kozaki, S., Sekine, Y., Takahashi, M., and Furuichi, T. (2006). Differential distributions of the $\mathrm{Ca} 2+-$ dependent activator protein for secretion family proteins (CAPS2 and CAPS1) in the mouse brain. J. Comp. Neurol. 495, 735-753.

Sadakata, T., Washida, M., Iwayama, Y., Shoji, S., Sato, Y., Ohkura, T., Katoh-semba, R., Nakajima, M., Sekine, Y., Tanaka, M., et al. (2007a). Autistic-like phenotypes in Cadps2-knockout mice and aberrant CADPS2 splicing in autistic patients. 117.

Sadakata, T., Washida, M., Morita, N., and Furuichi, T. (2007b). Tissue distribution of Ca2+-dependent activator protein for secretion family members CAPS1 and CAPS2 in mice. J. Histochem. Cytochem. 55, 301-311.

Sadakata, T., Kakegawa, W., Mizoguchi, A., Washida, M., Katoh-Semba, R., Shutoh, F., Okamoto, T., Nakashima, H., Kimura, K., Tanaka, M., et al. (2007c). Impaired cerebellar development and function in mice lacking CAPS2, a protein involved in neurotrophin release. J. Neurosci. 27, 2472-2482.

Sakaba, T., and Neher, E. (2001). Calmodulin Mediates Rapid Recruitment of Fast-Releasing Synaptic Vesicles at a Calyx-Type Synapse. Neuron 32, 1119-1131.

Sassa, T., Harada, S., Ogawa, H., Rand, J.B., Maruyama, I.N., and Hosono, R. (1999). Regulation of the UNC-18-Caenorhabditis elegans syntaxin complex by UNC-13. J. Neurosci. 19, 4772-4777.

Schägger, H. (2006). Tricine-SDS-PAGE. Nat. Protoc. 1, 16-22.

Scheuber, A., Rudge, R., Danglot, L., Raposo, G., Binz, T., Poncer, J.-C., and Galli, T. (2006). Loss of AP-3 function affects spontaneous and evoked release at hippocampal mossy fiber synapses. Proc. Natl. Acad. Sci. U. S. A. 103, 16562-16567.

Schikorski, T., and Stevens, C.F. (1997). Quantitative ultrastructural analysis of hippocampal excitatory synapses. J. Neurosci. 17, 5858-5867.

Schikorski, T., and Stevens, C.F. (2001). Morphological correlates of functionally defined synaptic vesicle populations. Nat. Neurosci. 4, 391-395.

Schmitz, F., Augustin, I., and Brose, N. (2001). The synaptic vesicle priming protein Munc13-1 is absent from tonically active ribbon synapses of the rat retina. Brain Res. 895, 258-263.

Schneggenburger, R., and Forsythe, I.D. (2006). The calyx of Held. Cell Tissue Res. 326, 311-337.

Schoch, S., Deák, F., Königstorfer, a, Mozhayeva, M., Sara, Y., Südhof, T.C., and Kavalali, E.T. (2001). SNARE function analyzed in synaptobrevin/VAMP knockout mice. Science 294, 1117-1122.

Schoch, S., Castillo, P.E., Jo, T., Mukherjee, K., Geppert, M., Wang, Y., Schmitz, F., Malenka, R.C., and Südhof, T.C. (2002). RIM1alpha forms a protein scaffold for regulating neurotransmitter release at the active zone. Nature 415, 321-326.

Segal, M., Vlachos, A., and Korkotian, E. (2010). The spine apparatus, synaptopodin, and dendritic spine plasticity. Neuroscientist 16, 125-131.

Seven, A.B., Brewer, K.D., Shi, L., Jiang, Q.-X., and Rizo, J. (2013). Prevalent mechanism of membrane bridging by synaptotagmin-1. Proc. Natl. Acad. Sci. U. S. A. 110, E3243-52. 
Shao, X., Fernandez, I., Südhof, T.C., and Rizo, J. (1998). Solution structures of the Ca2+-free and $\mathrm{Ca} 2+-$ bound $\mathrm{C} 2 \mathrm{~A}$ domain of synaptotagmin I: does $\mathrm{Ca} 2+$ induce a conformational change? Biochemistry 37, 16106-16115.

Shen, J., Tareste, D.C., Paumet, F., Rothman, J.E., and Melia, T.J. (2007). Selective activation of cognate SNAREpins by Sec1/Munc18 proteins. Cell 128, 183-195.

Shin, O.-H., Lu, J., Rhee, J.-S., Tomchick, D.R., Pang, Z.P., Wojcik, S.M., Camacho-Perez, M., Brose, N., Machius, M., Rizo, J., et al. (2010). Munc13 C2B domain is an activity-dependent Ca2+ regulator of synaptic exocytosis. Nat. Struct. Mol. Biol. 17, 280-288.

Siksou, L., Rostaing, P., Lechaire, J.-P., Boudier, T., Ohtsuka, T., Fejtová, A., Kao, H.-T., Greengard, P., Gundelfinger, E.D., Triller, A., et al. (2007). Three-dimensional architecture of presynaptic terminal cytomatrix. J. Neurosci. 27, 6868-6877.

Siksou, L., Varoqueaux, F., Pascual, O., Triller, A., Brose, N., and Marty, S. (2009). A common molecular basis for membrane docking and functional priming of synaptic vesicles. Eur. J. Neurosci. 30, 49-56.

Siksou, L., Triller, A., and Marty, S. (2011). Ultrastructural organization of presynaptic terminals. Curr. Opin. Neurobiol. 21, 261-268.

Siksou, L., Silm, K., Biesemann, C., Nehring, R.B., Wojcik, S.M., Triller, A., El Mestikawy, S., Marty, S., and Herzog, E. (2013). A role for vesicular glutamate transporter 1 in synaptic vesicle clustering and mobility. Eur. J. Neurosci. 37, 1631-1642.

De Simoni, A., Griesinger, C.B., and Edwards, F.A. (2003). Development of rat CA1 neurones in acute versus organotypic slices: role of experience in synaptic morphology and activity. J. Physiol. 550, 135147.

Smith, J.E., and Reese, T.S. (1980). Use of aldehyde fixatives to determine the rate of synaptic transmitter release. J. Exp. Biol. 89, 19-29.

Sørensen, J.B. (2009). Conflicting views on the membrane fusion machinery and the fusion pore. Annu. Rev. Cell Dev. Biol. 25, 513-537.

Sørensen, J.B., Nagy, G., Varoqueaux, F., Nehring, R.B., Brose, N., Wilson, M.C., and Neher, E. (2003). Differential control of the releasable vesicle pools by SNAP-25 splice variants and SNAP-23. Cell $114,75-86$.

Sørensen, J.B., Wiederhold, K., Müller, E.M., Milosevic, I., Nagy, G., de Groot, B.L., Grubmüller, H., and Fasshauer, D. (2006). Sequential N- to C-terminal SNARE complex assembly drives priming and fusion of secretory vesicles. EMBO J. 25, 955-966.

Southern, E.M. (1975). Detection of specific sequences among DNA fragments separated by gel electrophoresis. J. Mol. Biol. 98, 503-517.

Speidel, D., Varoqueaux, F., Enk, C., Nojiri, M., Grishanin, R.N., Martin, T.F.J., Hofmann, K., Brose, N., and Reim, K. (2003). A family of $\mathrm{Ca} 2+$-dependent activator proteins for secretion: comparative analysis of structure, expression, localization, and function. J. Biol. Chem. 278, 52802-52809.

Speidel, D., Bruederle, C.E., Enk, C., Voets, T., Varoqueaux, F., Reim, K., Becherer, U., Fornai, F., Ruggieri, S., Holighaus, Y., et al. (2005). CAPS1 regulates catecholamine loading of large dense-core vesicles. Neuron $46,75-88$.

Speidel, D., Salehi, A., Obermueller, S., Lundquist, I., Brose, N., Renström, E., and Rorsman, P. (2008). CAPS1 and CAPS2 regulate stability and recruitment of insulin granules in mouse pancreatic beta cells. Cell Metab. 7, 57-67. 
Steegmaier, M. (1998). Three Novel Proteins of the Syntaxin/SNAP-25 Family. J. Biol. Chem. 273, 34171-34179.

Stein, A., Weber, G., Wahl, M.C., and Jahn, R. (2009). Helical extension of the neuronal SNARE complex into the membrane. Nature 460, 525-528.

Stevens, C.F., and Tsujimoto, T. (1995). Estimates for the pool size of releasable quanta at a single central synapse and for the time required to refill the pool. Proc. Natl. Acad. Sci. U. S. A. 92, 846-849.

Stevens, D.R., and Rettig, J. (2009). The $\mathrm{Ca}(2+)$-dependent activator protein for secretion CAPS: do I dock or do I prime? Mol. Neurobiol. 39, 62-72.

Stevens, D.R., Wu, Z.-X., Matti, U., Junge, H.J., Schirra, C., Becherer, U., Wojcik, S.M., Brose, N., and Rettig, J. (2005). Identification of the minimal protein domain required for priming activity of Munc13-1. Curr. Biol. 15, 2243-2248.

Stoppini, L., Buchs, P.-A., and Muller, D. (1991). A simple method for organotypic cultures of nervous tissue. J. Neurosci. Methods 37, 173-182.

Südhof, T.C. (1995). The synaptic vesicle cycle: a cascade of protein-protein interactions. Nature 375, 645-653.

Südhof, T.C. (2004). The Synaptic Vesicle Cycle. Annu. Rev. Neurosci. 27, 509-547.

Südhof, T.C. (2012). The presynaptic active zone. Neuron 75, 11-25.

Südhof, T.C., and Rothman, J.E. (2009). Membrane fusion: grappling with SNARE and SM proteins. Science 323, 474-477.

Suh, Y.H., Terashima, A., Petralia, R.S., Wenthold, R.J., Isaac, J.T.R., Roche, K.W., and Roche, P.A. (2010). A neuronal role for SNAP-23 in postsynaptic glutamate receptor trafficking. Nat. Neurosci. 13, 338-343.

Sutton, R.B., Fasshauer, D., Jahn, R., and Brunger, A.T. (1998). Crystal structure of a SNARE complex involved in synaptic exocytosis at 2.4 A resolution. Nature $395,347-353$.

Takamori, S., Holt, M., Stenius, K., Lemke, E.A., Grønborg, M., Riedel, D., Urlaub, H., Schenck, S., Brügger, B., Ringler, P., et al. (2006). Molecular anatomy of a trafficking organelle. Cell 127, 831-846.

Tandon, A., Bannykh, S., Kowalchyk, J.A., Banerjee, A., Martin, T.F., and Balch, W.E. (1998). Differential regulation of exocytosis by calcium and CAPS in semi-intact synaptosomes. Neuron 21, $147-154$.

Tang, J., Maximov, A., Shin, O.-H., Dai, H., Rizo, J., and Südhof, T.C. (2006). A complexin/synaptotagmin 1 switch controls fast synaptic vesicle exocytosis. Cell 126, 1175-1187.

Toonen, R.F.G., Wierda, K., Sons, M.S., de Wit, H., Cornelisse, L.N., Brussaard, A., Plomp, J.J., and Verhage, M. (2006). Munc18-1 expression levels control synapse recovery by regulating readily releasable pool size. Proc. Natl. Acad. Sci. U. S. A. 103, 18332-18337.

Towbin, H. (1979). Electrophoretic Transfer of Proteins from Polyacrylamide Gels to Nitrocellulose Sheets: Procedure and Some Applications. Proc. Natl. Acad. Sci. 76, 4350-4354.

Ubach, J., Zhang, X., Shao, X., Südhof, T.C., and Rizo, J. (1998). Ca2+ binding to synaptotagmin: how many $\mathrm{Ca} 2+$ ions bind to the tip of a C2-domain? EMBO J. 17, 3921-3930. 
Varoqueaux, F., Sigler, A., Rhee, J.-S., Brose, N., Enk, C., Reim, K., and Rosenmund, C. (2002). Total arrest of spontaneous and evoked synaptic transmission but normal synaptogenesis in the absence of Munc13-mediated vesicle priming. Proc. Natl. Acad. Sci. U. S. A. 99, 9037-9042.

Varoqueaux, F., Sons, M.S., Plomp, J.J., and Brose, N. (2005). Aberrant morphology and residual transmitter release at the Munc13-deficient mouse neuromuscular synapse. Mol. Cell. Biol. 25, 59735984.

Vennekate, W., Schröder, S., Lin, C.-C., van den Bogaart, G., Grunwald, M., Jahn, R., and Walla, P.J. (2012). Cis- and trans-membrane interactions of synaptotagmin-1. Proc. Natl. Acad. Sci. U. S. A. 109, 11037-11042.

Verhage, M. (2000). Synaptic Assembly of the Brain in the Absence of Neurotransmitter Secretion. Science (80-. ). 287, 864-869.

Verhage, M., and Sørensen, J.B. (2008). Vesicle docking in regulated exocytosis. Traffic 9, 1414-1424.

Voets, T., Toonen, R.F., Brian, E.C., de Wit, H., Moser, T., Rettig, J., Südhof, T.C., Neher, E., and Verhage, M. (2001). Munc18-1 promotes large dense-core vesicle docking. Neuron 31, 581-591.

Walent, J.H., Porter, B.W., and Martin, T.F. (1992). A novel $145 \mathrm{kd}$ brain cytosolic protein reconstitutes $\mathrm{Ca}(2+)$-regulated secretion in permeable neuroendocrine cells. Cell 70, 765-775.

Walter, A.M., Wiederhold, K., Bruns, D., Fasshauer, D., and Sørensen, J.B. (2010). Synaptobrevin Nterminally bound to syntaxin-SNAP-25 defines the primed vesicle state in regulated exocytosis. J. Cell Biol. 188, 401-413.

Warming, S., Costantino, N., Court, D.L., Jenkins, N.A., and Copeland, N.G. (2005). Simple and highly efficient BAC recombineering using galK selection. Nucleic Acids Res. 33, e36.

Washbourne, P., Thompson, P.M., Carta, M., Costa, E.T., Mathews, J.R., Lopez-Benditó, G., Molnár, Z., Becher, M.W., Valenzuela, C.F., Partridge, L.D., et al. (2002). Genetic ablation of the t-SNARE SNAP-25 distinguishes mechanisms of neuroexocytosis. Nat. Neurosci. 5, 19-26.

Wassenberg, J.J., and Martin, T.F.J. (2002). Role of CAPS in dense-core vesicle exocytosis. Ann. N. Y. Acad. Sci. 971, 201-209.

Watanabe, S., Liu, Q., Davis, M.W., Hollopeter, G., Thomas, N., Jorgensen, N.B., and Jorgensen, E.M. (2013). Ultrafast endocytosis at Caenorhabditis elegans neuromuscular junctions. Elife 2, e00723e00723.

Weber, J.P., Reim, K., and Sørensen, J.B. (2010). Opposing functions of two sub-domains of the SNARE-complex in neurotransmission. EMBO J. 29, 2477-2490.

Weber, T., Zemelman, B. V, McNew, J.A., Westermann, B., Gmachl, M., Parlati, F., Söllner, T.H., and Rothman, J.E. (1998). SNAREpins: Minimal Machinery for Membrane Fusion. Cell 92, 759-772.

Weimer, R.M., Gracheva, E.O., Meyrignac, O., Miller, K.G., Richmond, J.E., and Bessereau, J.-L. (2006). UNC-13 and UNC-10/rim localize synaptic vesicles to specific membrane domains. J. Neurosci. $26,8040-8047$.

Wenzel, E.M., Morton, A., Ebert, K., Welzel, O., Kornhuber, J., Cousin, M.A., and Groemer, T.W. (2012). Key physiological parameters dictate triggering of activity-dependent bulk endocytosis in hippocampal synapses. PLoS One 7, e38188.

Wickelgren, W.O., Leonard, J.P., Grimes, M.J., and Clark, R.D. (1985). Ultrastructural correlates of transmitter release in presynaptic areas of lamprey reticulospinal axons. J. Neurosci. 5, 1188-1201. 
Wiederhold, K., Kloepper, T.H., Walter, A.M., Stein, A., Kienle, N., Sørensen, J.B., and Fasshauer, D. (2010). A coiled coil trigger site is essential for rapid binding of synaptobrevin to the SNARE acceptor complex. J. Biol. Chem. 285, 21549-21559.

De Wit, H., Cornelisse, L.N., Toonen, R.F.G., and Verhage, M. (2006). Docking of secretory vesicles is syntaxin dependent. PLoS One 1, e126.

De Wit, H., Walter, A.M., Milosevic, I., Gulyás-Kovács, A., Riedel, D., Sørensen, J.B., and Verhage, M. (2009). Synaptotagmin-1 docks secretory vesicles to syntaxin-1/SNAP-25 acceptor complexes. Cell 138, 935-946.

Wojcik, S.M., and Brose, N. (2007). Regulation of membrane fusion in synaptic excitation-secretion coupling: speed and accuracy matter. Neuron 55, 11-24.

Wojcik, S.M., Tantra, M., Stepniak, B., Man, K.-N.M., Müller-Ribbe, K., Begemann, M., Ju, A., Papiol, S., Ronnenberg, A., Gurvich, A., et al. (2013). Genetic Markers of a Munc13 Protein Family Member, BAIAP3, Are Gender Specifically Associated with Anxiety and Benzodiazepine Abuse in Mice and Humans. Mol. Med. 19, 135-148.

Wu, X.-S., McNeil, B.D., Xu, J., Fan, J., Xue, L., Melicoff, E., Adachi, R., Bai, L., and Wu, L.-G. (2009). $\mathrm{Ca}(2+)$ and calmodulin initiate all forms of endocytosis during depolarization at a nerve terminal. Nat. Neurosci. 12, 1003-1010.

Wu, Y., Gu, Y., Morphew, M.K., Yao, J., Yeh, F.L., Dong, M., and Chapman, E.R. (2012). All three components of the neuronal SNARE complex contribute to secretory vesicle docking. J. Cell Biol. 198, 323-330.

Xu, J., Pang, Z.P., Shin, O.-H., and Südhof, T.C. (2009). Synaptotagmin-1 functions as a Ca2+ sensor for spontaneous release. Nat. Neurosci. 12, 759-766.

Xu, J., Luo, F., Zhang, Z., Xue, L., Wu, X.-S., Chiang, H.-C., Shin, W., and Wu, L.-G. (2013). SNARE proteins synaptobrevin, SNAP-25, and syntaxin are involved in rapid and slow endocytosis at synapses. Cell Rep. 3, 1414-1421.

Xue, M., Reim, K., Chen, X., Chao, H.-T., Deng, H., Rizo, J., Brose, N., and Rosenmund, C. (2007). Distinct domains of complexin I differentially regulate neurotransmitter release. Nat. Struct. Mol. Biol. 14, 949-958.

Xue, M., Stradomska, A., Chen, H., Brose, N., Zhang, W., Rosenmund, C., and Reim, K. (2008). Complexins facilitate neurotransmitter release at excitatory and inhibitory synapses in mammalian central nervous system. Proc. Natl. Acad. Sci. U. S. A. 105, 7875-7880.

Xue, M., Lin, Y.Q., Pan, H., Reim, K., Deng, H., Bellen, H.J., and Rosenmund, C. (2009). Tilting the balance between facilitatory and inhibitory functions of mammalian and Drosophila Complexins orchestrates synaptic vesicle exocytosis. Neuron 64, 367-380.

Xue, M., Craig, T.K., Xu, J., Chao, H.-T., Rizo, J., and Rosenmund, C. (2010). Binding of the complexin $\mathrm{N}$ terminus to the SNARE complex potentiates synaptic-vesicle fusogenicity. Nat. Struct. Mol. Biol. 17, 568-575.

Yang, Y., and Calakos, N. (2011). Munc13-1 is required for presynaptic long-term potentiation. J. Neurosci. 31, 12053-12057.

Yang, X., Kaeser-Woo, Y.J., Pang, Z.P., Xu, W., and Südhof, T.C. (2010). Complexin clamps asynchronous release by blocking a secondary $\mathrm{Ca}(2+)$ sensor via its accessory a helix. Neuron 68 , 907-920. 
Yu, S.-C., Klosterman, S.M., Martin, A.A., Gracheva, E.O., and Richmond, J.E. (2013). Differential roles for snapin and synaptotagmin in the synaptic vesicle cycle. PLoS One 8, e57842.

Zhang, Q., Li, Y., and Tsien, R.W. (2009). The dynamic control of kiss-and-run and vesicular reuse probed with single nanoparticles. Science 323, 1448-1453.

Zhang, Y., Vilaythong, A.P., Yoshor, D., and Noebels, J.L. (2004). Elevated thalamic low-voltageactivated currents precede the onset of absence epilepsy in the SNAP25-deficient mouse mutant coloboma. J. Neurosci. 24, 5239-5248.

Zhao, S., Studer, D., Chai, X., Graber, W., Brose, N., Nestel, S., Young, C., Rodriguez, E.P., Saetzler, K., and Frotscher, M. (2012a). Structural plasticity of hippocampal mossy fiber synapses as revealed by high-pressure freezing. J. Comp. Neurol. 520, 2340-2351.

Zhao, S., Studer, D., Chai, X., Graber, W., Brose, N., Nestel, S., Young, C., Rodriguez, E.P., Saetzler, K., and Frotscher, M. (2012b). Structural plasticity of spines at giant mossy fiber synapses. Front. Neural Circuits 6, 103

Zhou, A., Brewer, K.D., and Rizo, J. (2013). Analysis of SNARE Complex/Synaptotagmin-1 Interactions by One-Dimensional NMR Spectroscopy. Biochemistry.

Zuber, B., Nikonenko, I., Klauser, P., Muller, D., and Dubochet, J. (2005). The mammalian central nervous synaptic cleft contains a high density of periodically organized complexes. Proc. Natl. Acad. Sci. U. S. A. 102, 19192-19197. 


\section{Acknowledgements}

I would like to express my gratitude towards all the people, who contributed to the success of this work and supported me during my PhD. Above all, I want to thank Prof. Dr. Nils Brose for including me as a member in his department and for his guidance, trust and constant encouragement.

I would specially like to thank Dr. Ben Cooper, who introduced me to world of electron microscopy, who helped and supported me throughout my PhD. His enthusiasm for science, his endless curiosity and above all technical knowledge has been crucial for the realization of the electron microscopy part of this project. I also want to express my sincere gratitude towards Dr. Jeong-Seop Rhee for introducing me to the organotypic slice culture system, and for his help, expertise and critical input during the project. Further, I am grateful to Dr. Sonja Wojcik, who designed the strategy for the Munc13-1 conditional knock-out mouse line, who patiently guided me through the molecular biology part of this study, and above all managed to constantly encourage me (even after yet another completely overexposed film after 5 month of Southern blotting).

I would like to thank my thesis committee members Prof. Dr. Reinhard Jahn and Prof. Dr. Stefan Eimer, who provided valuable input on the progress of my work. Further, I am extremely grateful to Dr. Dietmar Riedel, who kindly agreed to become an associative member of my thesis committee and whose constant help, methodological input, and regular (last-minute) access to his equipment were crucial for the realization of this study.

Further, I would like to thank our collaborators in Berlin (Prof. Dr. C. Rosenmund, Dr. M. Arancillo and Dr. Y.-J. Wu), in Stanford, California, USA (Prof. T.C. Südhof), in Amsterdam, The Netherlands (Dr. M. Loos and F. den Oudsten) and Göttingen (I. Herfort, Dr. U. Teichmann and T. Grundlach) for kindly providing mice and/or helping in establishing the genotyping here. I am also grateful to S.V. Dettmer and K. Treger for organizing and making mouse transports possible - even on very short notice!

I would also like to express my gratitude to the IMPRS MSc/PhD Neuroscience program and specially Prof. Dr. M. Hörner and S. Drube for their continuous help and support over the years. 
Moreover, I am sincerely grateful for the help of F. Benseler, C. Harenberg, D. Schwerdtfenger and I. Thanhäuser in the Institute DNA Core Facility and for the help of A. Ohle and J. Zimmermann from the animal facility. I also want to thank Dr. U. Fünfschilling and M. Schindler for the ES cell blastocyste injection and H. Horn, R. Merker, B. Beschke, and L. Demel from the IT department.

Further, I would like to acknowledge the support of all members of the molecular neurobiology department throughout the course of my $\mathrm{PhD}$ and I would specially thank Astrid, Ben, Bekir, Christoph, Hung-En, Ines, Kerstin, Klaus, Matthieu, Mimi, Noa, Ramya, Riikka, Sabine, Sally, and Sonja for their constant help, patience and encouragement. I am also grateful to S. Krinner for her help in characterizing the role of Complexins in synaptic vesicle docking during her lab rotation.

Last, but not least, I want to thank my family and friends for their emotional support. I am extremely grateful to my parents (all four of them), Ben, my brother and the 'Hilkes' - I promise I will be a better aunt from now on. I also want to thank Anna, Liesbeth and lonel and especially Julia, Natascha, Kristina and Katharina for all those years of loyal friendship.

I want to dedicate this work to two strong women, my grandparents Annelise Imig and Maria Weger, who taught me many valuable lessons in life and loved me unconditionally. 


\section{List of publications}

Imig, C., Min, S.-W., Krinner, S., Arancillo, A., Rosenmund, C., Südhof, T.C., Rhee, J.S., Brose, N., and Cooper, B. (2014) "The Morphological and Molecular Nature of Synaptic Vesicle Priming at Presynaptic Active Zones". Neuron, 84, 416-431.

Cooper, B., Hemmerlein, M., Ammermüller, J., Imig, C., Reim, K., Lipstein, N., Kalla, S., Kawabe, H. ,Brose, N., Brandstätter, J.H., and Varoqueaux, F. (2012). "Munc13-independent vesicle priming at mouse photoreceptor ribbon synapses." J Neurosci., 32, 8040-8052.

Burkhardt P, Stegmann CM, Cooper B, Kloepper TH, Imig C, Varoqueaux F, Wahl MC, and Fasshauer D (2011). "Primordial neurosecretory apparatus identified in the choanoflagellate Monosiga brevicollis." PNAS, 1073/pnas.1106189108.

Möbius W, Cooper B, Kaufmann A, Imig C, Ruhwedel T, Snaidero N, Saab AS, Varoqueaux F (2010). "Electron Microscopy of the mouse Central Nervous System." Methods Cell Biol, 96C:475-512. 